This item was submitted to Loughborough's Research Repository by the author.

Items in Figshare are protected by copyright, with all rights reserved, unless otherwise indicated.

\title{
OpenMolcas: From source code to insight
}

PLEASE CITE THE PUBLISHED VERSION

https://doi.org/10.1021/acs.jctc.9b00532

PUBLISHER

American Chemical Society (ACS)

VERSION

AM (Accepted Manuscript)

\section{PUBLISHER STATEMENT}

This document is the Accepted Manuscript version of a Published Work that appeared in final form in Journal of Chemical Theory and Computation, copyright @ A American Chemical Society after peer review and technical editing by the publisher. To access the final edited and published work see https://doi.org/10.1021/acs.jctc.9b00532

\section{LICENCE}

CC BY-NC-ND 4.0

\section{REPOSITORY RECORD}

Galván, Ignacio Fernández, Morgane Vacher, Ali Alavi, Celestino Angeli, Francesco Aquilante, Jochen Autschbach, Jie J Bao, et al.. 2019. "Openmolcas: From Source Code to Insight". figshare.

https://hdl.handle.net/2134/9884348.v1. 


\section{OpenMolcas: From Source Code to Insight}

Ignacio Fdez. Galván, Morgane Vacher, Ali Alavi, Celestino Angeli, Francesco Aquilante, Jochen Autschbach, Jie J. Bao, Sergey I. Bokarev, Nikolay A. Bogdanov, Rebecca K. Carlson, Liviu F. Chibotaru, Joel Creutzberg, Nike Dattani, Mickaël G. Delcey, Sijia Dong, Andreas Dreuw, Leon Freitag, Luis Manuel Frutos, Laura Gagliardi, Frédéric Gendron, Angelo Giussani, Leticia Gonzalez, Gilbert Grell, Meiyuan Guo, Chad E. Hoyer, Marcus Johansson, Sebastian Keller, Stefan knecht, Goran Kovačević, Erik Källman, Giovanni Li Manni, Marcus Lundberg, Yingjin Ma, Sebastian Mai, João Pedro Malhado, Per Åke Malmqvist, Philipp Marquetand, Stefanie A. Mewes, Jesper Norell, Massimo Olivucci, Markus Oppel, Quan Manh Phung, Kristine Pierloot, Felix Plasser, Markus Reiher, Andrew M. Sand, Igor Schapiro, Prachi Sharma, Christopher J. Stein, Lasse Kragh Sørensen, Donald G. Truhlar, Mihkel Ugandi, Liviu Ungur, Alessio Valentini, Steven Vancoillie, Valera Veryazov, Oskar Weser, Tomasz A. Wesołowski, Per-Olof Widmark, Sebastian Wouters, Alexander Zech, J. Patrick Zobel, Roland Lindh

Submitted date: 06/09/2019 Posted date: 06/09/2019

Licence: CC BY-NC-ND 4.0

Citation information: Fdez. Galván, Ignacio; Vacher, Morgane; Alavi, Ali; Angeli, Celestino; Aquilante, Francesco; Autschbach, Jochen; et al. (2019): OpenMolcas: From Source Code to Insight. ChemRxiv. Preprint.

In this article we describe the OpenMolcas environment and invite the computational chemistry community to collaborate. The open-source project already includes a large number of new developments realized during the transition from the commercial MOLCAS product to the open-source platform. The paper initially describes the technical details of the new software development platform. This is followed by brief presentations of many new methods, implementations, and features of the OpenMolcas program suite. These developments include novel wave function methods such as stochastic complete active space self-consistent field, density matrix renormalization group (DMRG) methods, and hybrid multiconfigurational wave function and density functional theory models. Some of these implementations include an array of additional options and functionalities. The paper proceeds and describes developments related to explorations of potential energy surfaces. Here we present methods for the optimization of conical intersections, the simulation of adiabatic and nonadiabatic molecular dynamics and interfaces to tools for semiclassical and quantum mechanical nuclear dynamics. Furthermore, the article describes features unique to simulations of spectroscopic and magnetic phenomena such as the exact semiclassical description of the interaction between light and matter, various X-ray processes, magnetic circular dichroism and properties. Finally, the paper describes a number of built-in and add-on features to support the OpenMolcas platform with post calculation analysis and visualization, a multiscale simulation option using frozen-density embedding theory and new electronic and muonic basis sets. 
File list (3)

v2_190906_main.pdf (10.70 MiB) view on ChemRxiv - download file

v2_190906_SI.pdf (12.53 MiB) view on ChemRxiv • download file

v2_190906_SI_input.zip (125.28 KiB) view on ChemRxiv - download file 


\section{OpenMolcas: From source code to insight}

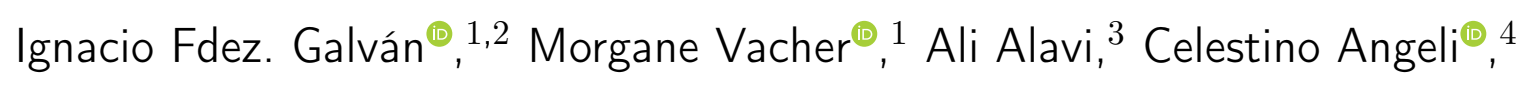

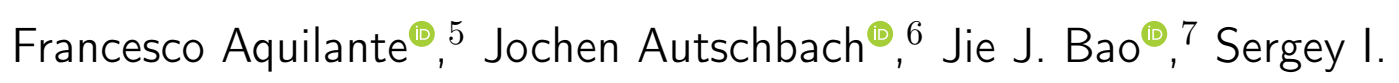

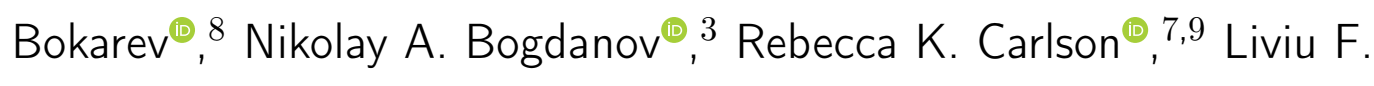
Chibotaru, ${ }^{10}$ Joel Creutzberg, ${ }^{11,12}$ Nike Dattani, ${ }^{13}$ Mickaël G. Delcey ${ }^{0}, 1$ Sijia S.

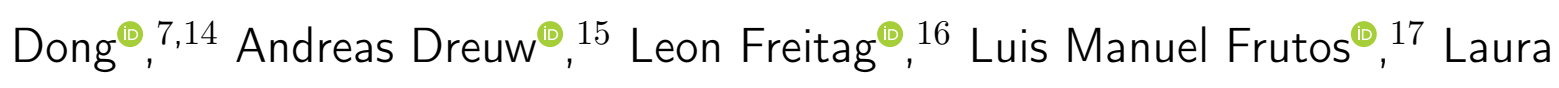

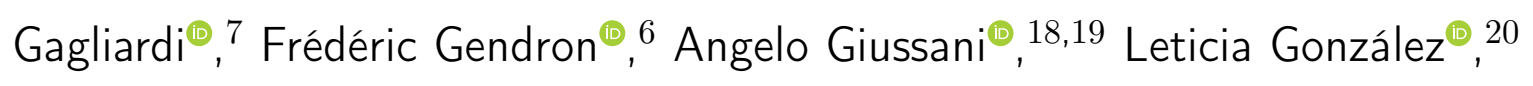

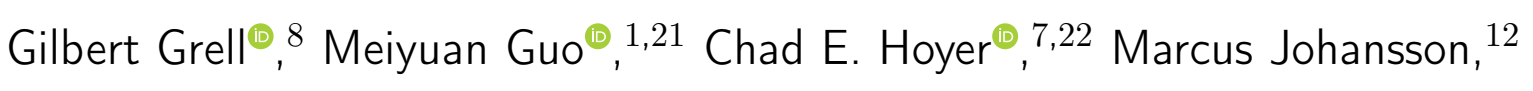
Sebastian Keller, ${ }^{16}$ Stefan Knecht四 ${ }^{16}$ Goran Kovačevićø ${ }^{23}$ Erik Källman, ${ }^{1}$

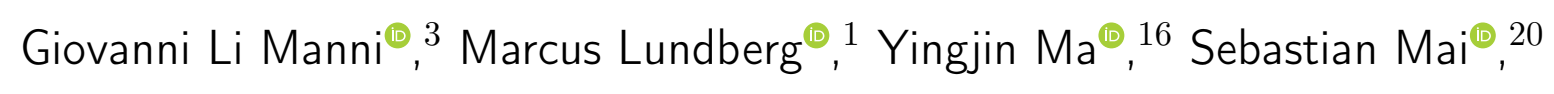
João Pedro Malhado, ${ }^{24}$ Per Åke Malmqvist॰ 12 Philipp Marquetand ${ }^{\oplus}, 20$

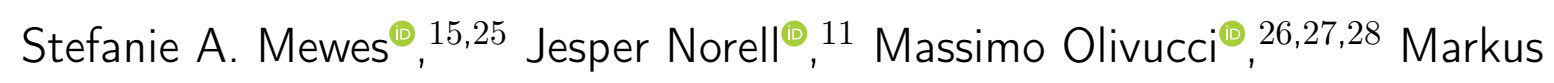

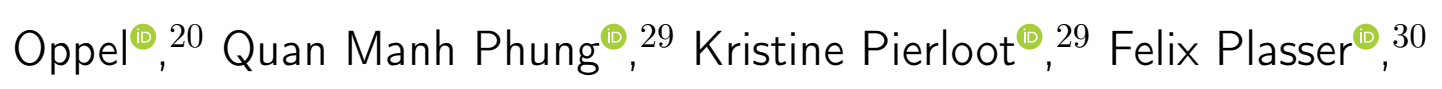

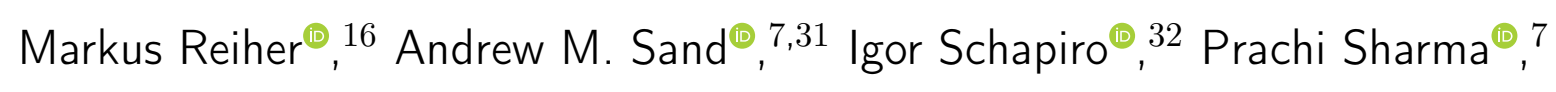
Christopher J. Stein®, 16,33 Lasse Kragh Sørensen॰1,34 Donald G. Truhlar॰7 Mihkel Ugandi॰ 1,35 Liviu Ungur ${ }^{\circ} 36$ Alessio Valentini॰ 37 Steven Vancoillie, ${ }^{12}$

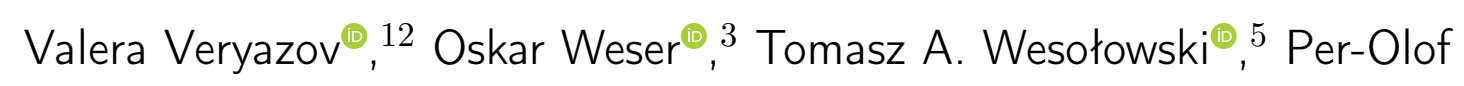
Widmark, ${ }^{12}$ Sebastian Wouters, ${ }^{38}$ Alexander Zech@, J. Patrick Zobel ${ }^{\oplus}{ }^{12}$ and Roland Lindh ${ }^{\circ * 2,39}$

1Department of Chemistry - Angström Laboratory, Uppsala University, P.O. Box 538, SE-751 21 Uppsala, Sweden 
2Department of Chemistry - BMC, Uppsala University, P.O. Box 576, SE-751 23

Uppsala, Sweden

3Max Planck Institut für Festkörperforschung, Heisenbergstraße 1, 70569 Stuttgart, Germany

4Dipartimento di Scienze Chimiche e Farmaceutiche, Università di Ferrara, Via Luigi Borsari 46, 44121 Ferrara, Italy

5Département de Chimie Physique, Université de Genève, 30 quai Ernest-Ansermet, CH-1211 Genève 4, Switzerland

6Department of Chemistry, University at Buffalo, State University of New York. Buffalo, NY 14260-3000, USA

7 Department of Chemistry, Chemical Theory Center, and Minnesota Supercomputing Institute, University of Minnesota, Minneapolis, MN 55455-0431, USA

8Institut für Physik, Universität Rostock, Albert-Einstein-Str. 23-24, 18059 Rostock, Germany

9Present address: Theoretical Division, Los Alamos National Laboratory, Los Alamos, $N M 87545, U S A$

10Theory of Nanomaterials Group, University of Leuven, Celestijnenlaan 200F, Leuven 3001, Belgium

11Department of Physics, AlbaNova University Center, Stockholm University, SE-106 91 Stockholm, Sweden

12Division of Theoretical Chemistry, Kemicentrum, Lund University, P. O. Box 124, SE-221 00 Lund, Sweden

13 Harvard Smithsonian Center for Astrophysics, Cambridge, Massachusetts, USA 14Present address: Materials Science Division and Center for Molecular Engineering, Argonne National Laboratory, Lemont, IL 60439, USA

15Interdisciplinary Center for Scientific Computing, Heidelberg University, Im Neuenheimer Feld 205 A, 69120 Heidelberg, Germany

16Laboratorium für Physikalische Chemie, ETH Zürich, Vladimir-Prelog-Weg 2, 8093 Zürich, Switzerland 
17Departamento de Química Analítica, Química Física e Ingeniería Química, and Instituto de Investigación Química "Andrés M. del Río". Universidad de Alcalá. E-28871 Alcalá de Henares, Madrid, Spain

18Department of Chemistry, University College London, 20 Gordon Street, London WC1H 0AJ, United Kingdom

19Instituto de Ciencia Molecular, Universitat de València, Apartado 22085, ES-46071 Valencia, Spain

20Institute of Theoretical Chemistry, Faculty of Chemistry, University of Vienna, Währinger Straße 17, 1090 Vienna, Austria

21Present address: Division of Chemical Physics, Chemical Center, Lund University, SE-221 00 Lund, Sweden

22Present address: Department of Chemistry, University of Washington, Seattle, WA 98195, USA

23Division of Materials Physics, Ruđer Bošković Institute, P.O.B. 180, Bijenička 54, HR-10002 Zagreb, Croatia

24Department of Chemistry, Imperial College London, London SW7 2AZ, United Kingdom

25Centre for Theoretical Chemistry and Physics, The New Zealand Institute for Advanced Study (NZIAS), Massey University Albany, Private Bag 102904, Auckland 0632, New Zealand

26Department of Biotechnology, Chemistry and Pharmacy, University of Siena, via A. Moro 2, 53100 Siena, Italy

27Department of Chemistry, Bowling Green State University, Bowling Green, OH

$$
\text { 43403, USA }
$$

28 USIAS and Institut de Physique et Chimie des Matériaux de Strasbourg, Université de Strasbourg-CNRS 67034 Strasbourg, France

29Department of Chemistry, KU Leuven, Celestijnenlaan 200F, Leuven 3001, Belgium 30Department of Chemistry, Loughborough University, Loughborough, LE11 3TU, United Kingdom 
31Present address: Department of Chemistry, Butler University, 4600 Sunset Avenue, Indianapolis, IN 46208, USA

32 Institute of Chemistry, The Hebrew University of Jerusalem, Jerusalem, Israel 33Present address: Chemical Sciences Division, Lawrence Berkeley National Laboratory,

1 Cyclotron Road, Berkeley CA 94720, USA

34Present address: Theoretical Chemistry and Biology, School of Engineering Sciences in Chemistry, Biotechnology and Health (CBH), Royal Institute of Technology (KTH), SE-106 91 Stockholm, Sweden

35Present address: Lehrstuhl für Theoretische Chemie, Ruhr-Universität Bochum, D-44780 Bochum, Germany 36 Department of Chemistry, National University of Singapore

37 Theoretical Physical Chemistry, Research Unit MolSys, Allée du 6 Août, 11, 4000 Liège, Belgium

38Brantsandpatents, Pauline van Pottelsberghelaan 24, 9051 Sint-Denijs-Westrem, Belgium

39 Uppsala Center for Computational Chemistry (UC $\left.C_{3}\right)$, Uppsala University, P.O. Box 596, SE-75124 Uppsala, Sweden

\title{
E-mail: roland.lindh@kemi.uu.se
}

\begin{abstract}
In this article we describe the OpenMolcas environment and invite the computational chemistry community to collaborate. The open-source project already includes a large number of new developments realized during the transition from the commercial MOLCAS product to the open-source platform. The paper initially describes the technical details of the new software development platform. This is followed by brief presentations of many new methods, implementations, and features of the OpenMolcas program suite. These developments include novel wave function methods such as stochastic complete active space self-consistent field, density matrix renormalization group (DMRG) methods, and hybrid multiconfigurational
\end{abstract}


wave function and density functional theory models. Some of these implementations include an array of additional options and functionalities. The paper proceeds and describes developments related to explorations of potential energy surfaces. Here we present methods for the optimization of conical intersections, the simulation of adiabatic and nonadiabatic molecular dynamics and interfaces to tools for semiclassical and quantum mechanical nuclear dynamics. Furthermore, the article describes features unique to simulations of spectroscopic and magnetic phenomena such as the exact semiclassical description of the interaction between light and matter, various X-ray processes, magnetic circular dichroism and properties. Finally, the paper describes a number of built-in and add-on features to support the OpenMolcas platform with post calculation analysis and visualization, a multiscale simulation option using frozen-density embedding theory and new electronic and muonic basis sets.

\section{Contents}

\begin{tabular}{lll}
\hline 1 & Introduction & 6
\end{tabular}

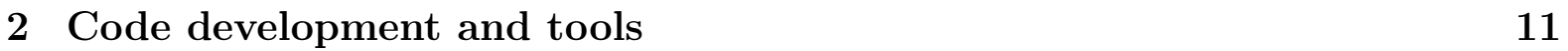

\begin{tabular}{lll}
\hline & Multiconfigurational wave function methods & 13
\end{tabular}

3.1 The Stochastic-CASSCF approach . . . . . . . . . . . . . . . . . . 15

3.2 Density matrix renormalization group interfaces . . . . . . . . . . . . . 21

3.3 Multiconfiguration pair-density functional theory . . . . . . . . . . . 34

$\begin{array}{|ll|}4 & \text { Exploration of potential energy surfaces }\end{array}$

4.1 Conical intersection optimization and characterization . . . . . . . . . . . 42

4.2 Dynamics simulations within OpenMolcas . . . . . . . . . . . . . 43

$4.3 \quad$ Nonadiabatic semiclassical dynamics interface . . . . . . . . . . . . . 46

4.4 Quantum dynamics interface . . . . . . . . . . . . . . . . . . 48

$\begin{array}{lll}5 & \text { Spectroscopic and magnetic properties } & 51\end{array}$

5.1 Light-matter interaction and beyond the multipole expansion . . . . . . 51 
$5.2 \quad$ X-ray spectroscopy and calculations of many core-hole excited states. . . $\quad 54$

5.3 Photoelectron spectroscopy and Dyson orbitals . . . . . . . . . . . . . 58

5.4 Magnetic circular dichroism spectroscopy . . . . . . . . . . . . . . 61

5.5 Molecular magnetic properties . . . . . . . . . . . . . . . . . . . . 64

5.6 Ultra-high precision computer spectroscopy. $\ldots \ldots \ldots$. . . . . . . . 67

\begin{tabular}{lll}
\hline 6 & Analysis and visualization & 68
\end{tabular}

6.1 Graphical interface and orbital visualization . . . . . . . . . . . . 69

6.2 Property integrals of individual orbitals . . . . . . . . . . . . . 71

6.3 Binatural or natural transition orbitals for excitation characterization . . 73

6.4 Detailed analysis of multireference wave functions . . . . . . . . . . . . . 74

\begin{tabular}{lll}
\hline 7 & Additional features & 77
\end{tabular}

$7.1 \quad$ Multiscale simulations by frozen-density embedding theory . . . . . . . . 77

7.2 Development of new ANO basis sets . . . . . . . . . . . . . . . . . . 79

7.3 Symmetrization of wave functions . . . . . . . . . . . . . . . . 80

7.4 Calculations of muonic atoms and molecules . . . . . . . . . . . . . 83

\begin{tabular}{lll}
\hline 8 & Summary & 84
\end{tabular}

\begin{tabular}{lr}
\hline Acknowledgement & 86
\end{tabular}

\begin{tabular}{ll}
\hline Supporting Information Available & 87
\end{tabular}

\begin{tabular}{lr}
\hline References & 88
\end{tabular}

\section{Introduction}

At the 4th MOLCAS developers' workshop, 30 March - 1 April 2016, in Vienna, Austria, the MOLCAS developer community decided that the source code of the MOLCAS project would be released, where the authors agree, as an open-source project under the GNU Lesser General Public License (LGPL) ${ }^{1}$ - the OpenMolcas project. This decision was followed by intensive work to adapt to this new format and context. An open-source 
repository was established September 2017. In late 2018 the transition to the new opensource format and integration platform was completed. The history of the OpenMolcas codebase dates back much earlier in time and for a summary of it we refer to previous MOLCAS publications..$^{2}$ Here we report on the new and recent developments and options available in OpenMolcas.

OpenMolcas is a software package capable of performing state-of-the-art quantum chemical calculations, but it is not the only one. Other packages, some of them open source, with similar or overlapping capabilities are (a necessarily nonexhaustive list): ACES,, 9 ADF, 10 BAGEL, 11 BigDFT, $\stackrel{12}{12}$ CFOUR, $\stackrel{13}{ }$ Columbus, 14 Dalton, 15

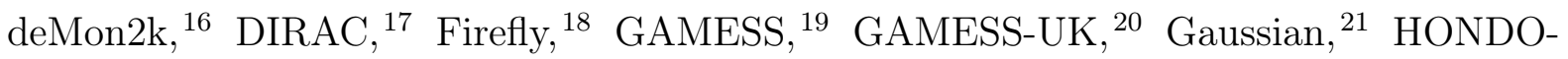

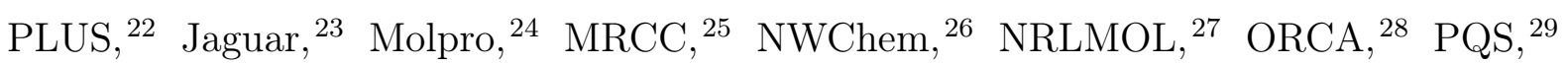

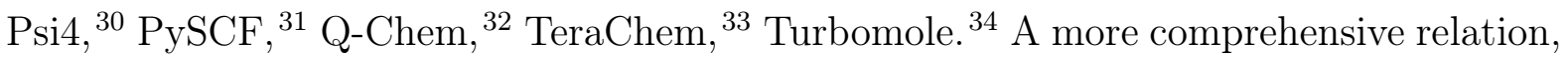
but restricted to open-source programs, can be found in ref. 35. A detailed comparison of the OpenMolcas features with these other packages is out of the scope of this report, but it can be stated that the main strength and focus of OpenMolcas is on multiconfigurational wave function methods, and applications and properties for which these methods are appropriately suited, as reflected in the contents of this article.

Apart from scientific and methodological improvements, the release of OpenMolcas incorporates a series of changes in code management and tools. These changes, as presented here, are aimed at an optimal interface for software accessibility for new developers and facilitating interaction with other codes. In this report the new developments are subdivided into five different categories: novel wave function and density functional methods, approaches to explore potential energy surfaces (PESs), implementations associated with various types of spectroscopy, tools for post analysis of orbitals and wave functions, and finally a set of some miscellaneous developments.

On the issue of resolving the prohibitive exponential scaling of the multiconfigurational wave function a number of new techniques has been introduced to eliminate this bottleneck. In particular, the Stochastic-CASSCF method has been implemented. Furthermore, two interfaces are introduced - with the CheMPS2 and QCMAQUIS programs 
- that greatly expand the potential of multiconfigurational wave functions by allowing much larger active spaces as well as speeding up calculations with usual active spaces. This is achieved by supplementing the full configuration interaction (full CI, FCI) solver in the RASSCF module by the density matrix renormalization group (DMRG). With these interfaces it is possible to perform DMRG-SCF, DMRG-CASPT2 and DMRG-NEVPT2 calculations, obtain analytical DMRG-SCF gradients or compute state interaction. Moreover, the multiconfiguration pair-density functional theory (MC-PDFT) option has been further developed. This includes translating several KS-DFT functionals not previously available, introducing analytical gradients for state-specific CAS-PDFT, state-interaction PDFT (SI-PDFT) for situations where two electronic states are strongly interacting and the use of PDFT in association with DMRG technology.

The exploration of the PESs is a significant part of any sophisticated tool for electronic structure calculations. In spite of being a leading package for the computational study of electronic excited states, MOLCAS has for a long time lacked the ability of computing nonadiabatic coupling vectors. The recent implementation of analytical nonadiabatic coupling vectors for SA-CASSCF wave functions is now available, together with practical methods for optimizing and characterizing conical intersections, and the use in simulations of nonadiabatic processes. Furthermore, simulating the time evolution of matter in the vicinity of electronic degeneracy regions is essential for understanding the rate and the product distribution of photochemical reactions and for complementing time-resolved experiments. Direct dynamics methods were developed to avoid the required computation and fitting of the PESs prior to dynamics simulations and to address the issue of the exponential scaling with the system size. OpenMolcas now supports three different tools for this. First, the DYNAMIX and SURFACEHOP modules of OpenMolcas allow molecular dynamics (MD) simulations of adiabatic and nonadiabatic processes; there are now options for generating starting conditions, adding external forces to the molecular system for steered MD simulations, and simulating isotope effects. Second, the SHARCOpenMolcas interface allows the simulation of nonadiabatic trajectory surface hopping dynamics including any arbitrary coupling; this means it can deal with photochemical 
events involving internal conversion and intersystem crossing on the same footing. Third, we report here a new interface to the Quantics package. Dynamics code for classical nuclei is rather straightforward since information is only needed at a given position in space; a quantum description of the nuclei has more problems because of delocalization of the wavepacket. The new interface with the Quantics code allows a quantum mechanical treatment of both electronic and nuclear dynamics via the direct-dynamics variational multiconfiguration Gaussian (DD-vMCG) method.

The OpenMolcas package can generate operator matrix elements between spin-orbitcoupled electronic states and is thus frequently used in simulation of various spectroscopic processes. Recently, these tools have been improved. For high-energy photons the short wavelength means that the electric field can no longer be treated as constant - as in the dipole approximation - and electric-dipole forbidden transitions can be observed. The solution, as presented here, is to either introduce higher orders in the multipole expansion or, more elegantly, use the exact exponential form of the wave vector. Core hole states are notoriously problematic to handle in standard computations, since these states are high up in energy. Now there is a simple option to use projection during the CI optimization to prevent the core hole being filled. Moreover, spectroscopy typically involves transitions between an initial state and several final states. The standard CI algorithms are designed to calculate ground and a relatively small number of low-lying excited states. However, to simulate experiments as X-ray spectroscopy, hundreds of high-energy states are needed. A new approach to the CI problem, which efficiently handles this case, is presented here. We report also the implementation of multiconfigurational Dyson orbitals for accurate simulations of molecular ionization processes. Another example is magnetic circular dichroism (MCD) as one of the prime tools to study the electronic states of complicated metal complexes and metalloproteins. Software tools have now been created for the easy generation of MCD spectral intensities with OpenMolcas, for molecules with degenerate ground states. We will also discuss the computation of magnetic properties for transition metal and lanthanide compounds. Moreover, an example in which the OpenMolcas platform has been used as an alternative to experiments, to generate micro-hartree accuracy of 
ionization potentials, is given.

Any quantum chemical simulation needs the support of post-calculation analysis tools to render the simulations to be more than just the matter of computing a numerical value of the energy or some other molecular property. Such tools enable the scientist to make qualitative conclusions from the simulations and to obtain insight in addition to quantitative predictions. They typically involve the generation of orbitals, which could be natural, canonical, or localized, and the graphical representation of these. In that respect, OpenMolcas has recently been enhanced with the following new utilities. The Graphical User Interface LUSCUS is a next generation of GV (Graphical Viewer) code; it is a lightweight viewer of input and output data produced by various quantum chemical codes, in particular the OpenMolcas program suite. One of the bottlenecks in the graphical visualization procedure is the size of the intermediate data. The ability to on-demand compute the data for visualization is now implemented in a stand-alone code, SAGIT. Furthermore, Pegamoid is a new orbital viewer that can read and write the native OpenMolcas orbital formats, simplifying the process of selecting and analyzing the active space for MCSCF calculations. Moreover, binatural orbitals, also called natural transition orbitals, are now available in order to characterize the nature of an electronic excitation. This can be computed by singular value decomposition of a transition density matrix using RASSCF wave functions in the RASSI module. Finally, the WFA (wave function analysis) module not only provides visualization tools but also computes quantitative descriptors, which allow for a rigorous and completely automatized analysis of excited-state computations.

In addition, as listed below, miscellaneous utilities and functionalities have been added to the program suite. The relativistic ANO-RCC basis set has been the spearhead of the MOLCAS/OpenMolcas program since its introduction a decade ago. It is now accompanied by an extremely small relativistic alternative, the ANO-XS basis set. The small size of ANO-XS greatly reduces integral computation times, yet still predicts, e.g., geometries or excitation energies with only small differences as compared to the larger ANO-RCC basis sets. Moreover, OpenMolcas is adapted to use point groups beyond $D_{2 \mathrm{~h}}$. Thus, for highly symmetrical molecules OpenMolcas can occasionally generate wave functions with 
broken symmetry. It is a problematic programming task to adapt a computational code to use higher point groups. However, a code for a posteriori symmetrization of the wave function, msym, has been developed and implemented in OpenMolcas. As a further development we report on the ability to simulate muonic atoms and molecules, i.e. systems containing one muon - a fermion with a mass about 207 times heavier than an electron. This includes developments of muonic basis sets, book keeping of two types of fermionic particles and basis sets in integral and wave function code, diagonal finite mass correction and analysis of parameters associated with the finite-nucleus approximation. Finally, a new method for multiscale electronic structure calculations has been implemented which is based on frozen-density embedding theory (FDET).

Thus, below we will in some details describe and discuss these new tools in the OpenMolcas program suite. The structure of the article will in all respects follow the order as presented above. This will be followed with a brief summary.

\section{Code development and tools}

The tools and culture in software development have changed dramatically in the last three decades since the initial versions of MOLCAS. The original code "would only run on the IBM 3090 computers under the JCL operating system", ${ }^{6}$ and although later versions made it more versatile and user-friendly, it remained for a long time a project developed mainly in Lund (Sweden), using a set of custom-written tools for debugging, configuring, updating and documenting the code. ${ }^{5}$ In the last few years, particularly since the establishment of annual MOLCAS developers workshops in 2013, the MOLCAS project has become a much more international and collaborative project, and has tried to keep up with times and embrace the new tools and technologies that are available for code management. Perhaps the most significant of the changes, and the one that triggers the need for this publication, is the choice of an open-source model, as discussed in the introduction.

The main codebase of OpenMolcas is written in Fortran language, something that 
reflects its history and background, and the popularity of Fortran in scientific programming. Newer code is increasingly written in other languages: a number of libraries and interfaces recently added to OpenMolcas are written in $\mathrm{C}$ or $\mathrm{C}++$, and the main input parser and driver has been rewritten in Python. The most significant changes introduced in OpenMolcas with respect to the development environment are the use of third-party open-source tools for code management, compilation and testing. Already in 2013 it was decided to use git $\frac{36}{36}$ as a version control system for MOLCAS, in connection with the release of OpenMolcas, it was considered important to have a source code repository that is publicly accessible, and not only for developers. As a build and configuration system,

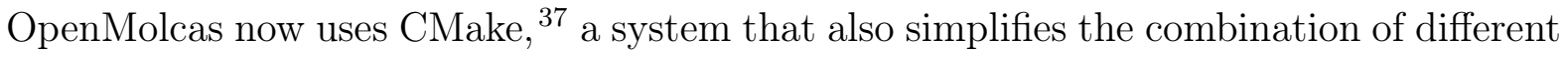
packages such as some of the interfaces reported in this paper. For testing, OpenMolcas uses the verification tools (test suite and scripts) that were already available in MOLCAS, but it additionally benefits from the continuous integration facilities in GitLab, $\frac{38}{\text { the cho- }}$ sen hosting platform, to ensure a fast and reliable test runs with minimal maintenance from OpenMolcas developers.

Historically, communication between the different programs in MOLCAS has been mostly done through files, in text or binary format. MOLCAS users will be familiar with names like "RunFile", "InpOrb" or "JobIph". These files have the inconvenience that they are specific to MOLCAS and in some cases incompatible between different versions and/or platforms. With the release of OpenMolcas there is also an increased effort to make use of more portable and standardized file formats for transferring data between programs and for storing the results of calculations - orbital and CI coefficients, geometries, energies. In particular, the most important results can now be stored in the HDF5 (hierarchical data format) format, ${ }^{39}$ which allows efficient storage and access of structured data, including its description. HDF5 files can then be accessed in any platform without knowledge of the program that generated them, since the size and structure of the data are also part of the format. We consider this an important step forward towards interoperability with other scientific codes. 


\section{Multiconfigurational wave function methods}

Important conceptual bases for understanding the motivations for some of the most important features of OpenMolcas are provided by the definition of configuration and the distinction between static correlation and dynamic correlation. A configuration is a particular way to assign electrons to orbitals, which may be double occupied, singly occupied, or unoccupied, and a configuration state function (CSF) is an approximate wave function with appropriate spin symmetry (singlet, doublet, etc.) and a definite set of orbital occupancies. Static correlation is also called near-degeneracy correlation, left-right correlation, and strong correlation, and it is due to the interaction of nearly degenerate configurations. A good zeroth-order wave function for a system with significant static correlation will have appreciable coefficients for two or more CSFs. Dynamic correlation is the rest of the correlation energy; it includes a variety of correlation effects ranging from very short range (due to the cusp in the correct many-electron wave function when two electrons approach one another) to very long range (as in dispersion interactions). The slow convergence of dynamic correlation with respect to adding more CSFs is often the bottleneck in making accurate calculations, and the most common way to calculate dynamic correlation is usually to optimize the orbitals with a self-consistent-field (SCF) calculation, and then include dynamic correlation by adding CSFs that are excited from a reference SCF wave function (single excitations, double excitations, triple excitations, etc.); the addition of excited CSFs may be done by perturbation theory, configuration interaction, or coupled cluster ${ }^{40-42}$ theory. When static correlation is negligible, good results can often be obtained with a single-CSF reference wave function (typically a Hartree-Fock,, $43-46$ $\mathrm{HF}$, wave function); methods employing this scheme are called single-reference methods. For strongly correlated systems, this method might be inaccurate unless one uses very high excitation levels (quadruple excitations or higher), which are often unaffordable. A more efficient way to treat strongly correlated systems is to use a multi-configuration reference wave function; such methods are called multi-reference methods. Systems well treated by single-reference methods with low levels of excitation are sometimes called single-reference systems, and systems that are much more efficiently treated with multi- 
configuration reference wave functions are sometimes called multi-reference systems or strongly correlated systems. Because many chemical systems of practical interest feature electronic structures dominated by more than one electronic configuration in their ground or excited states, as well as along reaction pathways in which they are involved, the development of efficient methods for multi-configuration wave functions and multi-reference correlation methods is a key challenge for quantum chemistry; it was one of the primary motivations for the original development of MOLCAS, and it remains a key strength of OpenMolcas. Multiconfigurational self-consistent field (MCSCF) methods, capable of dealing with these cases, have been known since the early days of quantum chemistry, but they gained popularity only when the complete active space self-consistent field method (CASSCF) was developed. $\stackrel{47+50}{50}$

Density functional theory presents a similar but conceptually distinct scenario. KohnSham density functional theory (KS-DFT) ${ }^{51}$ does not involve a wave function for the system under study, but it does involve a Slater determinant that has the same electron density as the system under study. This Slater determinant may be considered to be a reference wave function, and in that sense KS-DFT is a single-reference theory. Unlike Hartree-Fock theory, though, KS-DFT is in principle exact, in that there is an existence theorem for an exact density functional that would yield the correct electron density and energy. However, this density functional is unknown and probably unknowable. We must rely on approximate density functionals (ADFs), and currently available ADFs are less accurate for strongly correlated systems than for single-reference systems. $\frac{52[53}{}$ One way overcome this is to develop density functional theories that use a multiconfigurational reference wave functions, and OpenMolcas includes multi-configuration pair-density functional theory (MC-PDFT) $)^{54}$ as an example of this kind of theory.

$\operatorname{MOLCAS}^{8}$ (and now OpenMolcas) has always had a strong focus in multiconfigurational wave function methods and has pioneered the development of cutting-edge methods aiming at circumventing the exponential scaling limitation in CASSCF. The restricted active space (RAS) SCF,$\left[5566\right.$ the generalized active space (GAS) ${ }^{57} \mathrm{SCF}$ and the SplitGAS ${ }^{58}$ methods are examples. 
Multiconfigurational SCF methods such as the above, explicitly recover electron correlation within the active space and, via the self-consistent orbital relaxation procedure, part of the electron correlation outside the active space. In this respect it is to be noticed that the orbital relaxation in MCSCF procedures is not to be compared to the self-consistent orbital optimization in the single-reference HF procedure, as the MCSCF orbital gradient directly depends on the multiconfigurational wave function via the generalized Fock operator. Correlation effects not included at the MCSCF level are usually recovered by a posteriori treatments that use the preceding MCSCF wave function as reference. Examples of these methods within the OpenMolcas package are the multiconfiguration pair-density functional theory, MC-PDFT, $\underline{5459] 61}$ and second-order perturbation theory methods, such as CASPT2, RASPT2 ${ }^{62}$ and NEVPT2. ${ }^{65}$ Despite their broad applicability, these methods rely on the qualitative correctness of the reference wave function, or, in the case of MC-PDFT, its electron density, on-top pair-density, and the ratio of these two. $\frac{72}{72}$

The rest of this section describes with more detail some of the newest approaches implemented in OpenMolcas to work around the exponential scaling of CASSCF, while keeping its conceptual simplicity. In particular, the Stochastic-CASSCF method (section 3.1) uses a Monte-Carlo algorithm to solve the CI problem in the active space; the density matrix renormalization group (DMRG) - for which two different interfaces are presented (section 3.2) - expresses the wave function as a product of matrices, with a reduced number of free parameters with respect to a linear combination of configuration state functions (CSFs). In addition, the MC-PDFT capabilities in OpenMolcas (section 3.3) are enhanced with analytic gradients and state-interaction functionalities.

\subsection{The Stochastic-CASSCF approach}

The Stochastic-CASSCF $[3] \sqrt[76]{6}$ has been developed since 2015, initially for MOLCAS, ${ }^{8}$ and is now available in OpenMolcas. The method retains the simplicity of CASSCF, while circumventing the exponential scaling of the latter. This is obtained by replacing the Davidson diagonalization technique, ${ }^{[77}$ in its direct-CI implementation, ${ }^{[5178}$ with the full- 
CI quantum Monte-Carlo (FCIQMC) algorithm, used for the orbital optimization. Starting from the decoupled two-step Newton-Raphson method $\frac{88}{80}$ for the optimization of the orbitals and CI coefficients, the Stochastic-CASSCF procedure can be summarized in two conceptually simple steps - (i) the FCIQMC approach to optimize the CI-coefficients and obtain the one- and two-body density matrices and, (ii) the Super-CI algorithm for the orbital coefficients optimization. The Super-CI step is done according to standard procedures $\frac{47 / 48 / 50}{15}$ and will not be described here. The FCIQMC approach for the optimization of the CI coefficients, instead, will be described below in more detail.

The FCIQMC algorithm is a projector technique based on the imaginary-time Schrödinger equation

$$
\frac{\partial \Psi}{\partial \tau}=-\left(\hat{H}-E_{\mathrm{S}}\right) \Psi
$$

In this equation the term $E_{\mathrm{S}}$ is an energy offset, referred to as the shift, that is updated iteratively, converging to the ground-state correlation energy. In the context of FCIQMC, $E_{\mathrm{S}}$ plays the role of population control. Expanding the wave function, $\Psi(\tau)$, into a linear combination of Slater determinants and, integrating eq. (1), with a short time-step, $\Delta \tau$, such that the full propagator $e^{-\tau\left(\hat{H}-E_{\mathrm{S}}\right)}$ can be approximated to first-order, an iterable working equation is obtained

$$
C_{i}(\tau+\Delta \tau)=C_{i}(\tau)-\Delta \tau\left(H_{i i}-E_{\mathrm{S}}\right) C_{i}(\tau)-\Delta \tau \sum_{j \neq i} H_{i j} C_{j}(\tau)
$$

A deterministic update of the CI vector from the time $\tau$ to $(\tau+\Delta \tau)$ would require the storage of several arrays of the size of the CI vector and would face the same exponential scaling limitation of the standard direct-CI approach. Instead, in FCIQMC the concept of walkers is introduced. Walkers are signed Kronecker delta functions that reside on a specific determinant. We define $\delta_{i, i_{\alpha}}$ the $\alpha$-th walker residing on determinant $|i\rangle$. A sign is assigned to each walker, $s_{\alpha}= \pm 1$, associated with it. Thus the signed number of walkers, $N_{i}$, which reside on $|i\rangle$ is given by $N_{i}=\sum_{\alpha} s_{\alpha} \delta_{i, i_{\alpha}}$. The total number of walkers residing in all relevant determinants is kept to a fixed value, $N_{w}=\sum_{i}\left|N_{i}\right|$. This value 
is to be considered an optimization parameter in addition to the basis set and the active space. The larger the total number of walkers, the smaller the error due to the initiator approximation. $\stackrel{80}{ }$ In practice, this parameter is gradually increased until convergence over the energy estimate is reached.

The key point of FCIQMC is to allocate memory only for the populated determinants, instead of allocating memory for the entire CI vector. The purpose of FCIQMC is to generate distributions of walkers such that for a large number of total walkers and long imaginary time limit the expectation value of the number of walkers on each determinant becomes proportional to the CI coefficient of that determinant, $C_{i} \propto\left\langle N_{i}\right\rangle_{\tau}$. In FCIQMC, walker population dynamics simulates the imaginary-time evolution as given in eq. (2). The dynamics consists of three steps. In the spawning step child particles are generated from their parents into various locations of the Hilbert space. In the death step parents walkers are stochastically removed from the simulation. In the annihilation step parents and newly created walkers residing on the same determinant with opposite sign are eliminated.

The expectation value of the number of walkers on each determinant in practical calculations are never explicitly calculated. Instead derived properties are accumulated once the walker population has reached a dynamical equilibrium. One of these properties is the projected energy

$$
E_{\mathrm{P}}=\frac{\left\langle\sum_{i} N_{i}\left\langle D_{0}|\hat{H}| D_{i}\right\rangle\right\rangle_{\tau}}{\left\langle N_{0}\right\rangle_{\tau}},
$$

where the numerator and denominator are accumulated and averaged separately, $D_{0}$ is the reference determinant, and $\left\langle N_{0}\right\rangle_{\tau}$ is the time-averaged number of walkers on $D_{0}$. Oneand two-body reduced density matrices (RDMs) are similarly accumulated ${ }^{82}$ to evaluate the CASSCF energy as the expectation value of the Hamiltonian operator and to compute the matrices required for the orbital optimization step.

Electron correlation outside the active space, also known as dynamic correlation, can be recovered via MC-PDFT, 545961 using the Stochastic-CASSCF wave function as reference. 


\subsubsection{Technical details}

The Stochastic-CASSCF method in OpenMolcas relies on the interface of the NECI program, responsible for the FCIQMC CI algorithm, and the RASSCF program of OpenMolcas, responsible for the Super-CI method as an orbital optimizer. In principle, two installation protocols can be adopted that are referred to as embedded and uncoupled forms. In the embedded form, the NECI program is treated as a subroutine of the RASSCF program. This form effectively leads to an automatized version of the Stochastic-CASSCF within the OpenMolcas software. In the uncoupled form of Stochastic-CASSCF, NECI is installed as a stand-alone program and the OpenMolcas-NECI interface is controlled manually by the user. Dedicated keywords are required in the OpenMolcas input file to let OpenMolcas produce the relevant files, namely the file containing one- and twoelectron integrals in the MO basis (FCIDUMP), and an input file for the NECI program (see section S1). FCIQMC is then started externally, and the population is grown and stabilized before one- and two-body density matrices are accumulated. Walker population and other parameters can be tuned at this stage within the FCIQMC calculation if necessary. Density matrices are then transferred back to OpenMolcas and used to evaluate the Fock matrices necessary for the Super-CI step. After orbitals have been rotated, the integral file is updated and a new FCIQMC dynamics in the active space can be performed. The embedded OpenMolcas-NECI form is the recommended form for simple cases (small active spaces, small number of walkers, loose convergence). The uncoupled OpenMolcas-NECI form is the recommended form for difficult applications (large active spaces, large number of walkers, tight convergence).

Geometry, spatial symmetry, AO integral evaluation, scalar relativistic effects and additional external potentials are evaluated at the OpenMolcas level, and corrections are made to the FCIDUMP provided to the NECI program.

With the choice of the FCIQMC algorithm as CI eigensolver, the Stochastic-CASSCF method can be applied to larger active spaces, when compared to the deterministic analog. Active spaces containing up to 40 electrons and 38 orbitals have been reported. ${ }^{75}$ Integral evaluations and $\mathrm{AO}-\mathrm{MO}$ transformations can be carried via resolution-of-identity (RI) 
Cholesky decomposition (CD) techniques, $\frac{89}{93}$ available within the OpenMolcas package. The choice of the Super-CI method for the orbital relaxation together with the density fitting techniques enable Stochastic-CASSCF calculations easily with up to 5000 basis functions.

\subsubsection{Applications of the Stochastic-CASSCF method}

The Stochastic-CASSCF method has been applied to a number of chemical problems. Two very recent cases will be summarized in this section, namely the correlation mechanism leading to the large effective anti-ferromagnetic spin coupling, $J$, in corner-sharing cuprate solids and, the enhanced $\sigma$-donation $/ \pi$-back-donation mechanism that explains the stabilization of the intermediate spin-states over the high spin-states in Fe(II)-porphyrin model systems. For a more detailed description of these systems and the strategy used within the Stochastic-CASSCF framework we refer to the literature. 74 [76

Corner-sharing cuprates are of great interest, as they host high-temperature superconductivity upon doping. ${ }^{94}$ Magnetic properties of undoped cuprates have been rationalized on the basis of the Heisenberg-Dirac-Van Vleck Hamiltonian with anti-ferromagnetic nearest-neighbor magnetic coupling constant $J$. The values of $J$ in these systems are among the largest known, with some variations upon rather small geometrical differences. ${ }^{95}$ From a quantum-chemical standpoint understanding how $J$ directly depends on electron correlation is a challenging problem that has been investigated by several research groups. $\frac{96-100}{10 n v e n t i o n a l ~ w a v e ~ f u n c t i o n ~ t h e o r y ~ t r e a t m e n t ~ g r e a t l y ~ u n d e r e s t i m a t e s ~}$ the $J$ value. The Stochastic-CASSCF method has been used to investigate this aspect. A cluster containing two $\mathrm{CuO}_{4}\left(\right.$ or $\left.\mathrm{CuO}_{6}\right)$ units and all adjacent $\mathrm{Cu}^{2+}$ and $\mathrm{Sr}^{2+}$ (or $\mathrm{La}^{2+}$ ) ions has been employed in these calculations. The rest of the solid has been modeled by an array of point charges fitted to reproduce the Madelung potential due to infinite crystal in the cluster region. It has been demonstrated that to capture the essential elements of the super-exchange mechanism it is necessary to explicitly correlate the 20 valence $3 \mathrm{~d}$ and double-shell $\mathrm{d}^{\prime}$ orbitals on the $\mathrm{Cu}$ sites together with the six $2 \mathrm{p}$ and $\mathrm{p}^{\prime}$ orbitals on the bridging $\mathrm{O}$ ion, with a total of 24 electrons, resulting in a $\mathrm{CAS}(24,26)$. Calculations with 
this size of active space would be impossible with standard CASSCF technology, the corresponding CI wave function containing $\sim 9 \times 10^{13}$ Slater determinants. The calculation is carried out with ease using the Stochastic-CASSCF method. 76

Metal-porphyrins are crucial active sites in many enzymes responsible for electron transfer, oxygen transport and oxygen reduction. For example, the Fe(II)-porphyrin is the active species in cytochrome P450, that binds molecular oxygen and reduces it, leading to P450 Compound 1, responsible for the hydroxylation reaction (insertion of an oxygen atom in a $\mathrm{C}-\mathrm{H}$ bond) in alkyl chains. In spite of the numerous experimental and theoretical data, a definitive understanding of the ground-state electronic structure and its changes along the reaction pathway remain ambiguous, and an electronic explanation for the relative ordering of the low-lying spin-states remains unknown. Via large Stochastic-CASSCF $(32,34)$ calculations, $\underline{74}$ a complex mechanism has been proposed that demonstrates the differential stability of the intermediate triplet spin-state over the quintet spin-state in a model system of the Fe (II)-porphyrin. This mechanism involves ring correlation at the level of the $\pi$-system, a correlated breathing process at the metal center and charge-density redistribution. The large $(32,34)$ active space includes the entire $\pi$-system and nitrogen lone pairs, the $3 \mathrm{~d}$ and $\mathrm{d}^{\prime}$ orbitals at the metal center, and the $(4 \mathrm{~s} 4 \mathrm{p})$ shell. It has been demonstrated that ring correlation reduces the electron repulsion among the $\pi$ electrons, making the macrocycle a better electron acceptor. The correlating $\mathrm{d}^{\prime}$ orbitals provide the necessary flexibility for the orbital relaxation induced by charge-transfer processes from the lone-pairs of the ligand to the metal center. They also account for radial correlation, responsible for a lower on-site electron repulsion at the level of the metal center, and provide a larger overlap with the $\pi$ orbitals thus favoring charge-transfer from the metal center to the ligand. This complex mechanism can be related to a correlation enhanced $\sigma$-donation $/ \pi$-back-donation process and it is stronger for the triplet spin-state. It was suggested in ref. 74 that smaller active spaces, even upon second-order perturbative correction, such as in the conventional CASPT2 $(8,11)$ are not capable to fully capture this mechanism (see section 3.2 .5 for further discussion). The Stochastic-CASSCF technique has been also used to understand the role of semi-core 
electrons. This analysis has been performed via a large CAS(40,38) active space. $\frac{75}{75}$

\subsection{Density matrix renormalization group interfaces}

After White proposed the DMRG ${ }^{101 / 102}$ in 1992, subsequent work ${ }^{103}$ established that DMRG optimizes a matrix product state (MPS). The flexible nature of MPSs allows one to calculate excited states in an efficient manner $\stackrel{110}{ }$ and provides easy access to higherorder reduced density matrices and the evaluation of arbitrary correlation functions. The standard definition of an MPS describing a state over $L$ orbitals or sites is given by

$$
|\psi\rangle=\sum_{\sigma} \sum_{a_{1}, \ldots, a_{L-1}} M_{1 a_{1}}^{\sigma_{1}} M_{a_{1} a_{2}}^{\sigma_{2}} \cdots M_{a_{L-1} 1}^{\sigma_{L}}|\boldsymbol{\sigma}\rangle
$$

with basis states $|\boldsymbol{\sigma}\rangle=\left|\sigma_{1}, \ldots, \sigma_{L}\right\rangle$ and $\sigma_{l}=|\uparrow \downarrow\rangle,|\uparrow\rangle,|\downarrow\rangle,|0\rangle$ for spatial orbitals. In systems with symmetries, the matrix indices $a_{l}$ are complemented with a quantum number label $a_{l} \rightarrow q_{l} \alpha_{l}$, 111 leading to a block-sparse structure. Matrix product states will encode a full CI wave function exactly if the matrix dimension $m$ (the maximum size of the indices $a_{l}$, called "number of renormalized block states" or "bond dimension") is allowed to grow unrestrictedly. In practice, a finite $m$ is chosen which limits the amount of entanglement that the resulting MPS is able to describe between any bipartition of an $L$-orbital active space. For one-dimensional systems, so-called area laws ${ }^{112[113}$ prove that the amount of entanglement is constant irrespective of $L$ and that limiting $m$ is therefore a good approximation. For the general case in quantum chemistry, the convergence rate in $m$ depends on molecular topology and the choice of the orbital basis, influencing the sparsity of the one- and two-electron integrals.114

Analogously to MPS, matrix product operators (MPOs) ${ }^{110|115| 116}$ are defined as

$$
\widehat{\mathcal{W}}=\sum_{\boldsymbol{\sigma} \boldsymbol{\sigma}^{\prime}} \sum_{b_{1}, \ldots, b_{L-1}} W_{1 b_{1}}^{\sigma_{1} \sigma_{1}^{\prime}} \cdots W_{b_{l-1} b_{l}}^{\sigma_{l} \sigma_{l}^{\prime}} \cdots W_{b_{L-1}}^{\sigma_{L} \sigma_{L}^{\prime}}|\boldsymbol{\sigma}\rangle\left\langle\boldsymbol{\sigma}^{\prime}\right|
$$

The efficient determination of the $W$ matrices for Hamiltonian operators with long-range interactions such as Coulomb interactions is a nontrivial task.117 119 The DMRG algo- 
rithm formulated explicitly in terms of MPS and MPO is sometimes referred to as secondgeneration DMRG. Compared to the original formulation, a pure MPS/MPO implementation provides additional flexibility by allowing arithmetic operations to be performed on wave functions and operators independently. These properties are exploited, for instance, to implement a fully relativistic DMRG variant ${ }^{120}$ in QCMAQUIS (the first such implementation was presented earlier ${ }^{121}$ ), for interfacing with multireference perturbation theory ${ }^{122] 123}$ (see section 3.2.3) and for MPS state-interaction ${ }^{124}$ (see section 3.2.4).

This section presents the interface of OpenMolcas with two established DMRG codes: the CheMPS2 library, 125 a first-generation DMRG implementation, based on a MPS representation of wave functions (eq. (4)), and QCMAQUIs, 1111117126 a second-generation program that additionally makes use of a MPO representation of operators (eq. (5)). In a nutshell, both interfaces can replace the FCI solver from CASSCF with a DMRG implementation and be used to obtain state-specific or state-averaged DMRG-SCF wave functions, and perform single-state DMRG-CASPT2 calculations, $\stackrel{127}{ }$ while QCMAQUIS also supports DMRG-NEVPT2, MPSSI, and analytical state-specific gradients for DMRGSCF, support for the recently published analytical state-averaged DMRG-SCF gradients $\frac{128}{128}$ will be added in the near future.

The CheMPS2 library is a free open-source spin-adapted implementation of DMRG for $a b$ initio quantum chemistry. It is designed for high-performance computing, allowing for hybrid MPI and OpenMP parallellization, and can handle a general active space up to 40 electrons in 40 orbitals in a DMRG-SCF calculation. In a DMRG-CASPT2 calculation ${ }^{122|127| 129 \mid 130}$ requiring the 2- and 3-particle reduced density matrices (2- and 3-RDM) as well as the generalized Fock matrix contracted with the 4-RDM (F.4-RDM), an active space of $\sim 30$ active orbitals can be treated. Figure 1 illustrates a schematic representation of a DMRG-SCF/DMRG-CASPT2 calculation performed by the OpenMolcasCheMPS2 interface. DMRG-SCF calculations are performed in a manner similar to standard CASSCF calculations except that the number of renormalized state $m$ must be provided by the user. Additionally, other parameters controlling the cost and accuracy of the calculations, e.g. the initial guess of the matrix product state (MPS), the orbital ordering, 
the MPS checkpoint files, etc. can be optionally specified. The shared-memory (OMP) CheMPS2 binary is then called and executed. CheMPS2 outputs, such as the 2-RDM, are then fed to the RASSCF module, allowing the active orbitals to be optimized with the super-CI method. If a subsequent DMRG-CASPT2 calculation is required, the active orbitals are transformed into pseudocanonical orbitals in the last DMRG-SCF iteration, and the 3-RDM and F.4-RDM are calculated by CheMPS2 based on this pseudocanonical orbital basis. Because the generalized Fock matrix is diagonal in the pseudocanonical orbital basis, the calculation of the full 4-RDM is avoided, which drastically reduces the computational cost. 122

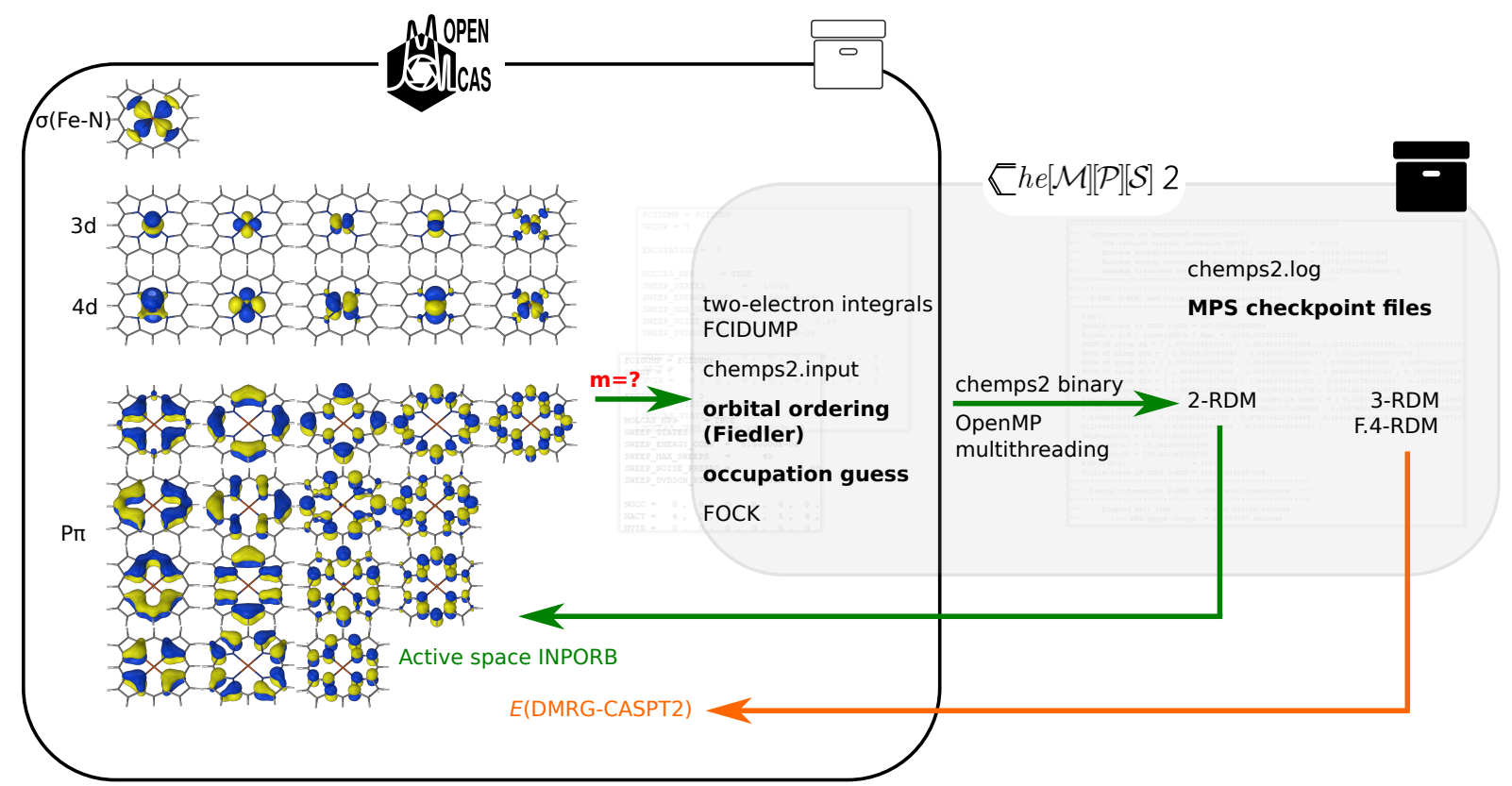

Figure 1: Schematic representation of a DMRG-SCF/DMRG-CASPT2 calculation performed by the OpenMolcas-CheMPS2 interface. Only the number of renormalized states $m$ must be specified. The user can define optional parameters to speed up the calculation, e.g. orbital ordering, occupation guess, the matrix product state (MPS) checkpoint files from a previous calculation, etc.

Regarding the other DMRG interface described in this paper, the OpenMolcasQCMAQUIS interface, it has been designed to (i) steer a DMRG calculation with the MPS/MPO program QCMAQUiS $\frac{111|117| 126}{1}$ and (ii) exchange data such as $N$-particle reduced density matrices ( $N$-RDMs), from QCMAQUIS to OpenMolcas. Figure 2 illustrates the capabilities of the OpenMolcas-QCMAQUIS interface. DMRG-SCF calculations with QCMAQUIS are enabled in OpenMolcas within the DMRGSCF module by specifying 
the ActiveSpaceOptimizer=QCMaquis keyword which sets QCMAQUIS as default active space solver. Note that the DMRGSCF module shares the same subset of keywords, e.g., for the wave function model specification, orbital-optimizer convergence acceleration, and MO analysis, that are available in OpenMolcas within the complete active space SCF optimizer module RASSCF. The core of the OpenMolcas-QCMAQUIS interface comprises a subset of Fortran90 modules which (i) "translate" in an automated fashion the userprovided wave function input data of OpenMolcas into the corresponding DMRG input for QCMAQUIS, (ii) generate on the fly the one- and two-electron integrals in the MO basis in the commonly used format defined by Knowles and Handy,131 (iii) invoke the actual DMRG wave function optimization with QCMAQUIS, and (iv) convert any (user)requested $N$-RDM or $N^{\prime}$-particle reduced transition density matrix (with $N=\{1,2,3,4\}$ and $\left.N^{\prime}=\{1,2,3\}\right)$ from QCMAQUis to OpenMolcas format. The complete MPS wave function, optimization input and output data as well as all molecular properties calculated by QCMAQUIS are stored in external files in HDF5 format and are therefore available in OpenMolcas for further external processing.

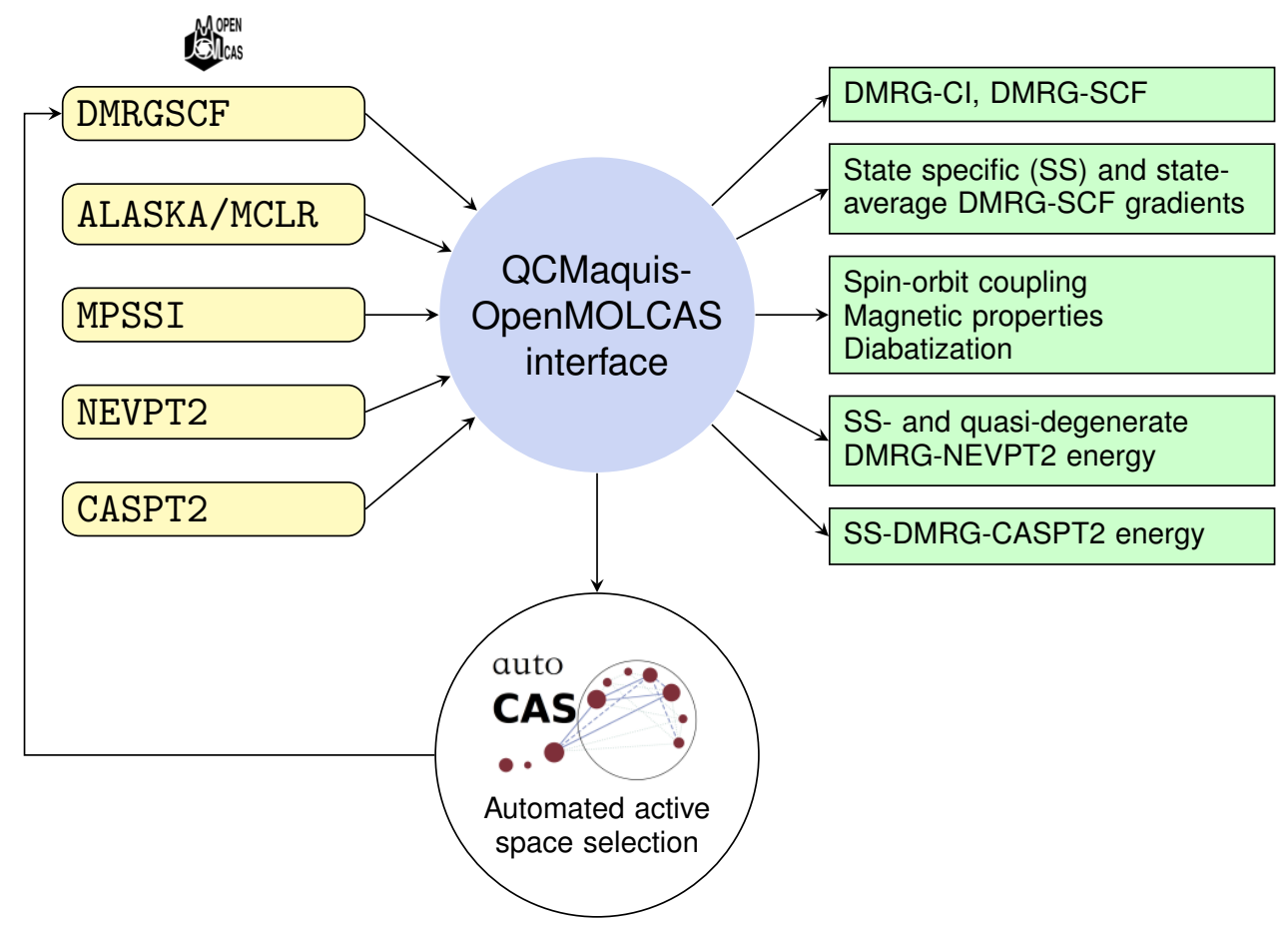

Figure 2: Schematic representation of the capabilities of the OpenMolcas-QCMAQUIS interface. OpenMolcas modules that are interfaced with QCMAQUIS are listed on the left, and the properties that can be obtained are listed on the right. 


\subsubsection{Active space selection based on entanglement measures}

The selection of an appropriate active space for multiconfigurational calculations is a task as important as it is difficult. While several guidelines exist for this selection, $, 132,136$ it still requires expertise with trial and error and, hence, constitutes a considerable entry barrier to such calculations. ${ }^{137}$ An automated protocol has been proposed ${ }^{138}$ and implemented in combination with the OpenMolcas-QCMAQUIS interface, which selects orbitals for the active space based on orbital entanglement entropies ${ }^{139 \mid 140}$ from a partially converged DMRG calculation. This protocol has become possible owing to (i) the feature of DMRG that allows one to consider large active spaces in an exploratory fashion through limiting the number of sweeps and the block dimension and owing to (ii) the spread of entanglement measures defined for one or a pair of orbitals so that rather robust thresholds can be defined that separate strongly from weakly correlated orbitals; the former to be chosen for an active space in a final well-converged DMRG or CASSCF calculation.

The orbital entanglement entropies are calculated from the one- and two-orbital reduced density matrices. The elements of these matrices can be expressed as expectation values of strings of creation and annihilation operators and are efficiently calculated within a DMRG algorithm. The explicit equation for the single-orbital entropy, on which the selection protocol is based, reads

$$
s_{i}(1)=-\sum_{\alpha=1}^{4} w_{\alpha, i} \ln w_{\alpha, i},
$$

whereas the two-orbital entropy follows in close analogy as

$$
s_{i j}(2)=-\sum_{\alpha=1}^{16} w_{\alpha, i j} \ln w_{\alpha, i j} .
$$

In these equations, $w_{\alpha, i}$ and $w_{\alpha, i j}$ are the eigenvalues of the one- and two-orbital reduced density matrices, respectively, $i, j$ are orbital indices, and $\alpha$ is one of the four (or 16) possible occupations of a spatial orbital (or a pair of spatial orbitals). Both quantities can be combined to give the mutual information which quantifies the entanglement of an 
orbital pair

$$
I_{i j}=\frac{1}{2}\left[s_{i}(1)+s_{j}(1)-s_{i j}(2)\right]\left(1-\delta_{i j}\right) .
$$

Both the mutual information and the single-orbital entropies are collected in entanglement diagrams such as the one in fig. 3 that provide an intuitive interpretation of the orbital entanglement.

It was demonstrated that CAS wave functions calculated from these automatically selected active spaces are suitable first-order wave functions for subsequent CASPT2 calculations. $\frac{141}{14}$ Furthermore, consistent active spaces for several electronic states and along reaction coordinates can be identified ${ }^{142}$ The automated active orbital space selection has been implemented as a graphical user interface (GUI) called AUTOCAS ${ }^{143[144}$ that drives OpenMolcas and QCMAQUIS. AUTOCAS is the first computer program that allows one to run multiconfigurational calculations, for which an active space must be selected, in a fully automated way. It can be downloaded separately free of charge from the SCINE webpage. 145

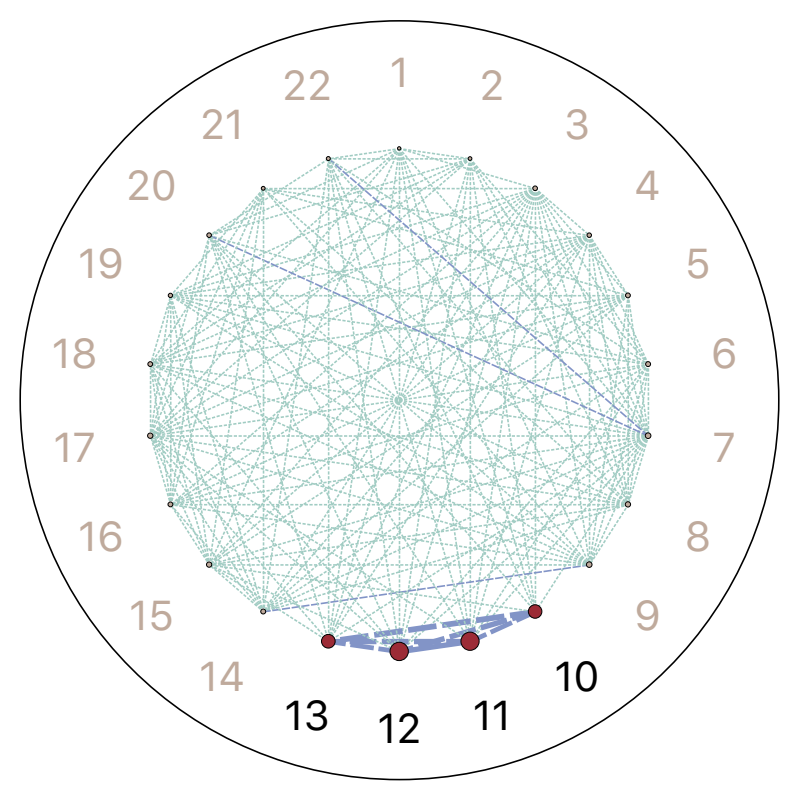

Figure 3: Entanglement diagram obtained from a DMRG wave function optimized with the QCMAQUIS program with $m=250$ and 10 sweeps including all valence orbitals of butadiene that were obtained from a minimal basis Hartree-Fock calculation. A circle with an area proportional to the single-orbital entropy is associated with each orbital. The thickness of the connecting lines is proportional to the mutual information of an orbital pair. The $\pi$-orbitals are those with numbers $10-13$ and would be selected by the automated protocol as implemented in AUTOCAS. 


\subsubsection{Analytic ground- and excited-state DMRG-SCF gradients}

A central feature of the traditional multiconfigurational wave function toolbox of OpenMolcas is the availability of analytic energy gradients (with respect to the nuclear coordinates) for a single ("state-specific") or an ensemble ("state-averaged") of electronic states. $146[148$ State-specific and state-averaged gradients are, for instance, key for the calculation of ground- and excited-state equilibrium structures, reaction paths, and for molecular dynamics simulations. Likewise, excited-state gradients are vital for modeling a wide range of photochemical phenomena, such as photochemical and photophysical pathways or resonance Raman spectra. Whereas a state-specific DMRG-SCF gradient is rather straightforward to implement,, 149 a formalism for state-averaged DMRG-SCF gradient theory has only been recently presented and applied to an optimization of a conical intersection in the study of the photochemistry of 1,2-dioxetanone. ${ }^{128}$ The applicability of state-specific DMRG-SCF gradients has been demonstrated for optical spectroscopy, in particular, for resonance Raman spectroscopy, 150 and that of the state-averaged DMRGSCF gradients in a conical intersection optimization. 128

In analogy to CASSCF, the calculation of a state-specific DMRG-SCF analytic gradient, available with the OpenMolcas-QCMAQUIS interface, requires the one- and twoparticle reduced density matrices for a given target state in addition to the derivatives of the Hamiltonian, i.e., the derivatives of one- and two-electron integrals, with respect

to the nuclear coordinates. $\frac{149 \mid 150}{14}$ The latter quantities can be calculated independently of the parent multiconfigurational wave function. Their calculation is carried out most efficiently in the AO-basis (to which the reduced density matrices are backtransformed from the MO basis) which is implemented in the ALASKA module of the OpenMolcas framework.

\subsubsection{Perturbation theory with DMRG reference}

While DMRG-SCF is capable of describing static correlation for large active spaces, a significant contribution to electron correlation required for quantitative results, namely the dynamic correlation, must be accounted for separately. Traditionally, second-order pertur- 
bation theory with a zeroth-order Hamiltonian that describes the static correlation effects has been most popular (but coupled-cluster models combined with a DMRG reference have also been considered ${ }^{151}[153)$. Multiconfigurational second-order perturbation theory in the form of CASSCF/CASPT2 became the flagship method of the (Open)Molcas quantum chemistry package. ${ }^{\frac{154}{1}}$ In the early 2000s, Angeli, Cimiraglia, Malrieu et al. .65 introduced another formulation of a CAS-based multireference perturbation theory, the (second-order) $n$-electron valence state perturbation theory (NEVPT2). The main difference of NEVPT2 with respect to CASPT2 is the definition of the zeroth-order Hamiltonian: NEVPT2 uses the Dyall Hamiltonian, $\frac{155}{15}$ wich explicitly considers two-electron interactions among the electrons in the active space, as a model Hamiltonian to define the zeroth-order Hamiltonian. The two-electron interactions in the reference ensure that NEVPT2 is intruder-state free and size consistent. ${ }^{71}$ NEVPT2 allows for two different contraction schemes to construct the first-order wave function: the partially contracted (PC) scheme is identical to the one in CASPT2, while the strongly contracted (SC) scheme introduces additional contractions, further simplifying the formalism.

The majority of the DMRG-based perturbation-theory methods encompass DMRGCASPT2 2 (available with both the OpenMolcas-CheMPS2 and OpenMolcasQCMAQUIS interfaces) and DMRG-NEVPT2 2 (available with the OpenMolcasQCMAQUIS interface). One of the major drawbacks of these approaches (or rather of the internal contraction formalism $\frac{157-159}{159}$ on which these theories are based) is their dependence on higher-order $N$-RDMs $(N>2)$, whose evaluation scales as $\mathcal{O}\left(L^{2 N}\right)$ with the number of active orbitals $L$. For instance, in case of the four-particle RDM, its evaluation scales formally as $\mathcal{O}\left(L^{8}\right)$. While for small active spaces (small $L$ ) this is not a problem, the fourth-order RDM evaluation becomes quickly the computational bottleneck for active spaces of more than 20 to 22 orbitals.

Apart from the RDM scaling problem, the ability to deal with large basis sets is crucial for increasing active space sizes. In particular, the transformation of the two-electron integrals from the AO to the MO basis becomes a bottleneck. One of the flagship features of the OpenMolcas program package is the Cholesky decomposition of the two-electron 
integral matrix, initially proposed by Beebe and Linderberg 160 and developed over the years by Aquilante, Lindh, Pedersen and co-workers for various single- and multireference electronic structure methods. $\frac{90931161162}{19}$ The DMRG-NEVPT2 implementation in QCMAQUIS $^{123}$ and the DMRG-CASPT2 calculations with the OpenMolcas-CheMPS2 and OpenMolcas-QCMAQUIS interfaces take full advantage of the Cholesky decomposition to handle the two-electron integrals $\frac{162}{162}$ and the DMRG reference wave function (CD-DMRG-NEVPT2, CD-DMRG-CASPT2).

\subsubsection{State interaction for matrix product states}

In the restricted active space state-interaction (RASSI) approach of Malmqvist, ${ }^{163}$ sets of spin-free (SF) eigenstates can be coupled under the influence of the spin-orbit (SO) coupling and a variety of highly important molecular properties can be evaluated. A critically important feature of the RASSI program is that the state interaction can be performed among different sets of SF states with different spin or spatial symmetry, with the molecular orbitals (MOs) optimized for each set of states individually. This implies mutual nonorthogonality of the respective MO bases and requires the transformation of pairs of MO sets to a bi-orthonormal basis. $\frac{163}{1}$ These nonunitary transformations require a counter-transformation of the representations of the wave functions such that the physical content of the latter remains intact. This feature was, until recently, only available for CI-type wave functions but not for MPSs.

The formalism and implementation for MPS-based state interaction (MPSSI) within QCMAQUIS $\frac{124}{124}$ includes the critical capability to work with nonorthogonal MO sets. The approach differs considerably from Malmqvist's but is likewise based on constructing biorthogonal MO bases and concomitant counter-transformations of the MPSs. An MPSSI flowchart for generating SO-coupled wave functions and matrix elements of operators for molecular properties is shown in fig. 4. Very importantly, the MPSs serve as a complete substitute for the CI-type states in calculations of SO-coupled wave functions as well as SF and SO molecular properties with the RASSI program. For instance, ref. 124 reported relative $\mathrm{SF}$ and $\mathrm{SO}$ valence state energies and state compositions for $\mathrm{Te}_{2}, \mathrm{NpO}_{2}{ }^{2+}$, 


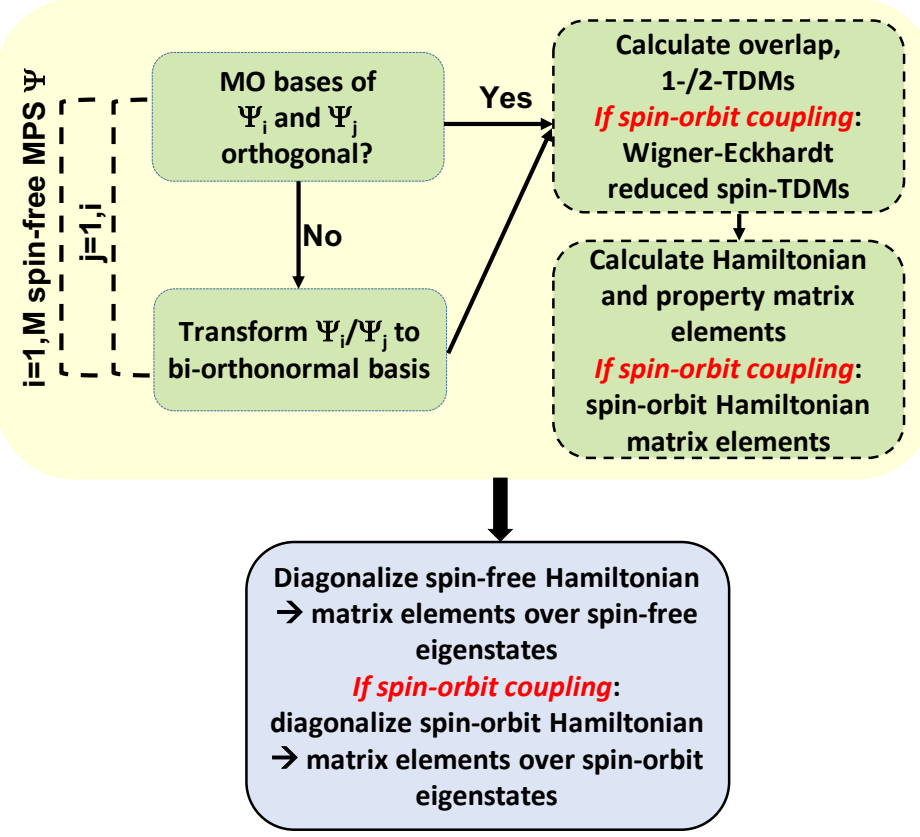

Figure 4: Flowchart illustrating the MPS state-interaction (MPSSI) approach to calculate property matrix elements of spin-free and spin-orbit coupled MPS wave functions according to ref. 124.

and $\mathrm{PuO}_{2}^{2+}$, as well as EPR $g$-factors for the actinyl species, from MPS-SO calculations with QCMAQUIS and the RASSI program. The MPSSI results were in full agreement with CASSCF and CASPT2. $\frac{164}{1}$ Therefore, the full suite of SO-level molecular properties and spectra previously available in the RASSI program can now be calculated with the potentially very large active spaces that are accessible with MPS wave functions.

\subsubsection{Applications of DMRG-MRPT2}

The case of the spin states of Fe(II)-porphyrin (FeP) has already been mentioned in the context of Stochastic-CASSCF (section 3.1.2), where it was proposed (for a smaller model system) that a small $\mathrm{CAS}(8,11)$ active space might be insufficient and a much larger active space, including all ligand $\pi$ as well as extra nitrogen orbitals and the 4s4p shell on the metal center, is needed. ${ }^{74}$ Here we discuss a series of DMRG-SCF and DMRG-CASPT2 calculations performed on the lowest triplet $\left({ }^{3} \mathrm{~A}_{2 g}\right)$ and quintet $\left({ }^{5} \mathrm{~A}_{1 g}\right)$ states of $\mathrm{FeP}$ with the OpenMolcas-CheMPS2 interface. The active space used is CAS $(26,27)$, containing, next to the $\mathrm{CAS}(8,11)$ space, $16 \mathrm{P}\left(\pi, \pi^{*}\right)$ orbitals (all but the $2 \mathrm{p}_{z}$ of the $\beta$ carbons of the pyrrole rings). The best DMRG calculations, using $m=10000$ for the $\operatorname{CAS}(26,27)$ active 
space differ by less than $0.4 \mathrm{~kJ} / \mathrm{mol}$ from the $\mathrm{CAS}(8,11)$ results, giving a quintet-triplet gap of $-68.53 \mathrm{~kJ} / \mathrm{mol}$ with DMRG-SCF and $-19.46 \mathrm{~kJ} / \mathrm{mol}$ with DMRG-CASPT2. The conclusion is that including the $\mathrm{P}\left(\pi, \pi^{*}\right)$ orbitals on top of the CAS $(8,11)$ space has a minor impact on the CASSCF/CASPT2 description of the spin state energetics in FeP. In refs. 165,166 it was shown that an improved description should instead come from more extensive basis sets (extrapolated to the complete basis set limit) as well as a special $(\mathrm{CCSD}(\mathrm{T}))$ treatment of the Fe $(3 \mathrm{~s}, 3 \mathrm{p})$ semi-core electrons. All details of the calculations, a plot of the active natural orbitals and a discussion on the convergence and performance can be found in the SI.

Another example of the OpenMolcas-CheMPS2 interface shown in the SI is the geometry optimization of ferrocene $\left(\mathrm{Fe}\left(\mathrm{C}_{5} \mathrm{H}_{5}\right)_{2}\right.$ or $\left.\mathrm{FeCp}_{2}\right)$ at DMRG-CASPT2 level, this demonstrates that the interface can easily be coupled with other modules that require either the 2-RDM or CASPT2 total energy. The equilibrium structure of $\mathrm{FeCp}_{2}$ was optimized (with numerical gradients) employing both standard CASPT2, with a CAS $(10,10)$ active space, and DMRG-CASPT2, with an extended CAS $(14,18)$ active space. The number of renormalized states $m$ is 1000, which gives converged DMRG-CASPT2 energies for medium-sized active spaces. $\frac{129}{}$ Further computational details and active natural orbitals can be found in the SI. The key structural parameters of ferrocene, calculated with CASPT2, DMRG-CASPT2, and CCSD $(\mathrm{T})^{167}$ are shown in table 1. Interestingly, the results indicate that CASPT2 and DMRG-CASPT2 give almost the same structure. As compared to CASPT2, DMRG-CASPT2 predicts slightly longer $\mathrm{Fe}-\mathrm{C}$ and $\mathrm{Fe}-\mathrm{Cp}$ bond distances by less than $0.005 \AA$. The results imply that in this simple case, the size of the active space has a minor impact on the optimized geometry. As compared to the CCSD(T) results, $\frac{167}{167}$ both CASPT2 and DMRG-CASPT2 predict the same $d(\mathrm{C}-\mathrm{C})$ and $d(\mathrm{C}-\mathrm{H})$, whereas they underestimate the $\mathrm{Fe}-\mathrm{C}$ and $\mathrm{Fe}-\mathrm{Cp}$ bond distances by $\sim 0.03 \AA$. All calculations were performed on a single Intel(R) Xeon(R) CPU E5-2680 v3@ $2.50 \mathrm{GHz}$ machine, 256 GB RAM, and 16 MPI processes. A numerical gradient calculation with the $\operatorname{CAS}(10,10)$ active space finishes in around 2 hours, while it takes $\sim 9$ hours for the DMRG CAS $(14,18)$ active space. For comparison purposes, a standard CASPT2 calcula- 
tion employing the large active space requires a fairly large amount of memory $(\sim 20 \mathrm{~GB}$ per process), consequently, only 12 MPI processes can fit the machine, and a numerical gradient calculation takes almost 8 days with this setting. It is also worth noting that a geometry optimization cycle using $\operatorname{CCSD}(\mathrm{T})$ analytical gradients can take almost 9 days. 167

Table 1: Structural parameters of the eclipsed conformation of ferrocene ${ }^{a}$

\begin{tabular}{lccc}
\hline & CASPT2 $^{b}$ & DMRG-CASPT2 $^{c}$ & ${\text { CCSD }(\mathrm{T})^{d}}^{d}$ \\
\hline$d(\mathrm{Fe}-\mathrm{Cp})(\AA)$ & 1.610 & 1.615 & 1.648 \\
$d(\mathrm{Fe}-\mathrm{C})(\AA)$ & 2.016 & 2.020 & 2.047 \\
$d(\mathrm{C}-\mathrm{C})(\AA)$ & 1.426 & 1.426 & 1.427 \\
$d(\mathrm{C}-\mathrm{H})(\AA)$ & 1.077 & 1.076 & 1.079 \\
$\angle(\mathrm{Cp}-\mathrm{H})(\text { deg. })^{e}$ & 0.89 & 1.08 & 0.52 \\
\hline
\end{tabular}

${ }^{a}$ In CASPT2, DMRG-CASPT2, and CCSD(T) calculations, cc-pwCVTZ basis set (672 basis functions) was used, all 96 electrons were correlated.

${ }^{b}$ Standard CASPT2 calculation with CAS $(10,10)$.

${ }^{c}$ DMRG-CASPT2 $[m=1000]$ calculation with CAS(14,18).

${ }^{d}$ Ref. 167.

${ }^{e}$ Angle between hydrogens and cyclopentadienyl ring, positive values means hydrogen is bent towards metal.

As an example of a DMRG-NEVPT2 calculation with the OpenMolcas-QCMAQUIS interface, we show here results of CD-DMRG-NEVPT2 for the energy gaps between the lowest quintet, triplet, and singlet states of a large iron(II) complex, $\mathrm{FeN}_{2} \mathrm{C}_{72} \mathrm{H}_{100}$, shown in fig. 5. This complex was chosen by the Neese group in reference calculations ${ }^{168}$ for their DLPNO-NEVPT2 approach $\frac{169}{169}$ and by Coughtrie et al. in their paper on embedded multireference coupled cluster theory. ${ }^{[170}$ As the PC-variant of the CD-DMRG-NEVPT2 approach is known to be unreliable for large active spaces and small bond dimensions $m$ (due to the incomplete convergence of the zeroth-order wave function), 123 the CD-DMRGSC-NEVPT2 variant was applied, given that it is much less sensitive to the convergence of the zeroth-order wave function. The active orbitals for the initial DMRG-SCF step were determined with the automated active space selection protocol ${ }^{138}$ using the AutoCAS program (see section 3.2.1). The active spaces for all spin states must be equal and, according to the selection protocol, comprise the union of the active spaces selected for the individual spin states. In this way, 17 orbitals were selected by the protocol, resulting 
in an active space of 14 electrons in 17 orbitals. However, upon orbital optimization of the triplet wave function, one orbital rotated out of the active space, and hence, the active spaces for different spin states would no longer be consistent, so the orbital was removed from the active space for all spin states, resulting in an active space of 14 electrons in 16 orbitals. In all calculations, the maximum bond dimension was set to $m=512$. Although this is a rather small value for the bond dimension, it was found to be a suitable approximation in certain cases, $\frac{123 \mid 156}{12}$ especially if an accuracy of about $8 \mathrm{~kJ} / \mathrm{mol}$ is considered to be sufficient. Further computational details are available in the SI.
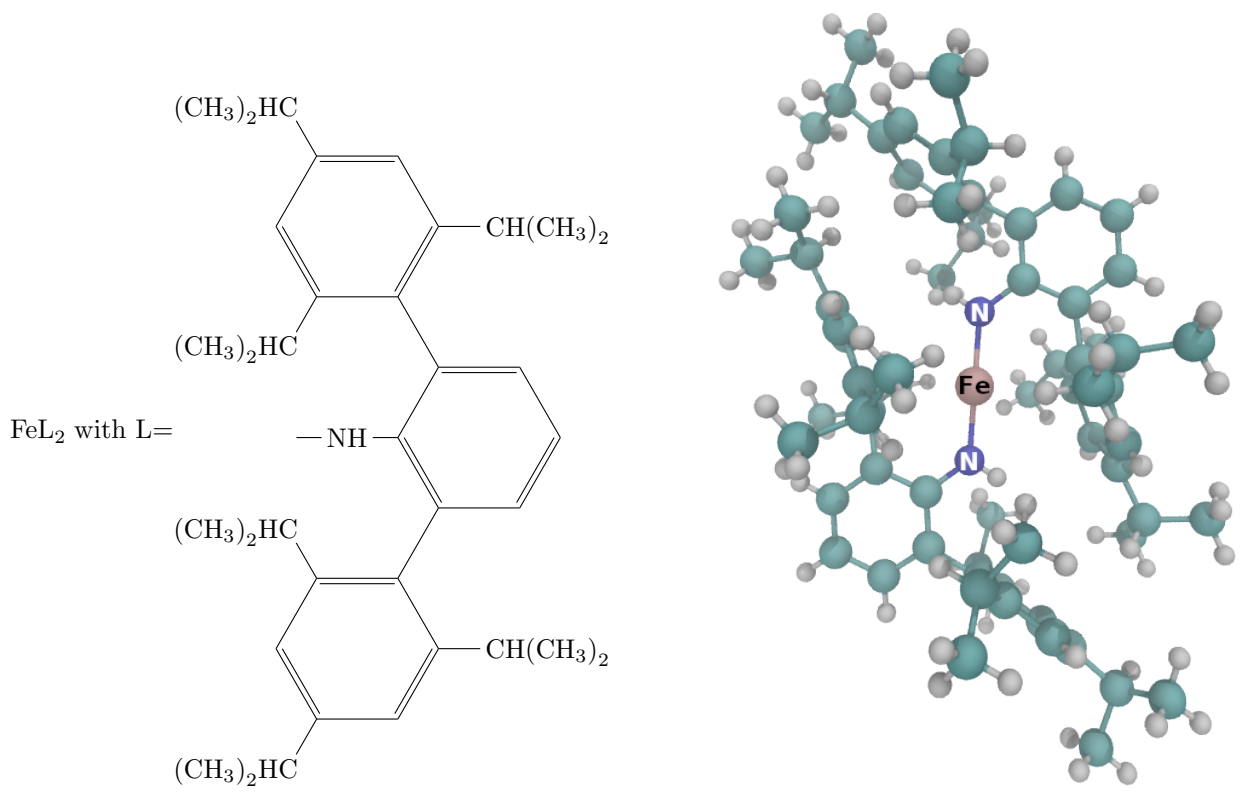

Figure 5: Lewis and molecular structure of the $\mathrm{FeN}_{2} \mathrm{C}_{72} \mathrm{H}_{100}$ complex in ref. 169 (Cartesian coordinates were taken from that reference). Color code: turquoise - carbon, white hydrogen.

Table 2: Excitation energy in $\mathrm{kJ} / \mathrm{mol}$ relative to the quintet $\left({ }^{5} \mathrm{~A}\right)$ ground state of $\mathrm{FeN}_{2} \mathrm{C}_{72} \mathrm{H}_{100}$. Results in refs. 169 and 170 were obtained with a CAS(6,5) (and with a def2-TZVP basis set), whereas we chose a CAS(14,16) (and an ANO basis set; see text for further discussion) for the CD-DMRG-SC-NEVPT2 and DMRG-SCF calculations. "emb." abbreviates "embedded in".

\begin{tabular}{cccccccc}
\hline & $\begin{array}{c}\text { DMRG-SC- } \\
\text { NEVPT2 }\end{array}$ & $\begin{array}{c}\text { SC- } \\
\text { NEVPT2 }\end{array}$ & $\begin{array}{c}\text { DLPNO-SC- } \\
\text { NEVPT2 }\end{array}$ & $\begin{array}{c}\text { NEVPT2 } \\
\text { emb. } \\
\text { CASSCF }\end{array}$ & $\begin{array}{c}\text { ic-MRCCSD(T) } \\
\text { emb. } \\
\text { PNO-CASPT2 }\end{array}$ & CASSCF & $\begin{array}{c}\text { DMRG- } \\
\text { SCF }\end{array}$ \\
St. & this work & ref. 169 & ref. 169 & ref. 170 & ref. 170 & ref. 170 & this work \\
\hline${ }^{3}$ A & 165.8 & 177.2 & 178.6 & 178.4 & 176.6 & 218.6 & 146.3 \\
${ }^{1} \mathrm{~A}$ & 241.7 & - & - & 290.3 & 262.4 & 346.1 & 197.8 \\
\hline
\end{tabular}


Table 2 contains the quintet-triplet and quintet-singlet excitation energies calculated with CD-DMRG-SC-NEVPT2 and DMRG-SCF along with results from refs. 169 and 170 obtained with several NEVPT2 and embedded MRCC variants. The quintet-triplet gap of $165.8 \mathrm{~kJ} / \mathrm{mol}$ deviates only by approximately $10 \mathrm{~kJ} / \mathrm{mol}$ from the embedded ic$\operatorname{MRCCSD}(\mathrm{T})$ result from ref. 170 which may be attributed to the different basis sets (ANO-RCC-VDZP/VQZP vs. def2-TZVP) and the different active orbital spaces from which the reference wave function was constructed. The quality of the reference wave function may be assessed from a comparison of the CASSCF and DMRG-SCF excitation energies with the final results that include the NEVPT2 contribution: DMRG-SCF excitation energies show a significantly smaller deviation from the DMRG-SCF plus NEVPT2 results than their CASSCF counterparts, which indicates that NEVPT2 benefits from an improved active space for the reference wave function calculation and that this can be provided by the suggested automated active-orbital selection algorithm. However, the quintet-singlet excitation shows a much larger discrepancy between the DMRG-NEVPT2 results and those from the literature. First of all, the CASSCF result for the small active space deviates even more from the DMRG-SCF result than what was observed for the quintet-triplet excitation. Unfortunately, no NEVPT2 reference data are available for this higher excitation. Still, the embedded NEVPT2 result from Coughtrie et al. 170 is about $30 \mathrm{~kJ} / \mathrm{mol}$ larger that the embedded MRCC result, whereas they were very similar in the singlet-triplet case. This may also be taken as an indication that the CAS of the reference was too small because the MRCC approach may compensate for a small-CAS reference wave function through the coupled-cluster excitation hierarchy. Accordingly, the DMRG-NEVPT2 result obtained for the larger active space is only about $20 \mathrm{~kJ} / \mathrm{mol}$ smaller than the embedded MRCC result.

\subsection{Multiconfiguration pair-density functional theory}

Multiconfiguration pair-density functional theory (MC-PDFT) ${ }^{54161}$ combines the advantages of wave function theory and density functional theory to provide a method for efficient evaluation of the electronic energy of strongly correlated systems. In MC-PDFT 
the first step is to calculate a multiconfiguration wave function, and in OpenMolcas,

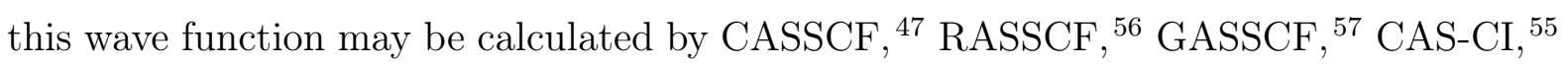
RAS-CI, ${ }^{[5]}$ GAS-CI, 171 stochastic-CASSCF ${ }^{73}$ (section 3.1), and DMRG $101|102| 109|172| 173$ (section 3.2. CASSCF, RASSCF, and GASSCF are special cases of the multiconfiguration self-consistent-field (MCSCF) method, and one can use either state-specific $\underline{174}$ SCF or state-averaged $\frac{175 / 176}{S C F}$. The total MC-PDFT energy is expressed as

$$
E=V_{\mathrm{nn}}+\left\langle\Psi^{\mathrm{MC}}|\hat{T}| \Psi^{\mathrm{MC}}\right\rangle+V_{\mathrm{ne}}+V_{C}+E_{\mathrm{ot}}[\rho, \Pi]
$$

where the first term corresponds to the nuclear-nuclear interaction; the second, the third, and the fourth terms, respectively, correspond to the kinetic energy, the nuclear-electron attraction, and the classical Coulomb interaction of the electronic charge cloud with itself; and the final term is the on-top energy. In eq. (9) the kinetic energy and the classical electrostatic energy (nuclear-nuclear repulsion energy, nuclear-electron attraction energy, and classical electron-electron Coulomb energy) are directly obtained from the multiconfigurational wave function, $\Psi$, while the rest of the electronic energy is calculated from an on-top density functional that is a functional of the electron density $\rho$ and on-top pair density $\Pi$ (which represents the probability of finding two electrons on top of each other) of that reference wave function.

Computationally, eq. (9) reduces to

$$
E=V_{\mathrm{nn}}+\sum_{p q} h_{p q} D_{p q}+\frac{1}{2} \sum_{p q r s} g_{p q r s} D_{p q} D_{r s}+E_{\mathrm{ot}}[\rho, \Pi, \nabla \rho, \nabla \Pi]
$$

where $\boldsymbol{D}$ is the one-electron density matrix, $\boldsymbol{h}$ and $\boldsymbol{g}$ contain respectively the one- and two-electron integrals, and $p, q, r$, and $s$ are general orbital indices including inactive and active orbitals. An MC-PDFT calculation involves two steps: (1) calculation of the multiconfiguration wave function and (2) calculation of the on-top density functional energy. Because the post-self-consistent-field step is a calculation based on a density functional, the computational effort for step 2 is generally negligible compared to the cost of step 1, and this makes MC-PDFT computationally more affordable than other 
multireference methods such as those based on perturbation theory or multireference configuration interaction.

An MC-PDFT calculation always uses multiconfiguration wave functions that are spin eigenfunctions. MC-PDFT is free from delocalization error, $\frac{177}{17}$ which is sometimes considered to be the most fundamental source of error in KS-DFT with approximate exchange-correlation functionals. MC-PDFT is also less prone than KS-DFT to selfinteraction error. 178

The decomposition of the MC-PDFT energy expression of eq. (9) into its individual components is sometimes important for understanding chemical and physical phenomena in molecular systems. ${ }^{721179}$ This decomposition is available in OpenMolcas, along with the tabulation of $\rho$ and $\Pi$ in Cartesian coordinates for plotting and visualization.

\subsubsection{On-top functionals for MC-PDFT}

Due to the symmetry dilemma, the spin-polarized exchange-correlation functionals of KS-DFT are not compatible with the spin-densities of a multiconfiguration wave function. To bypass the symmetry dilemma, one uses translated ${ }^{54}$ and fully translated 6 on-top functionals, which are obtained by translation of KS-DFT exchange-correlation functionals of the spin densities and their gradients. Translated functionals are denoted with the prefix "t," and fully translated functionals are denoted with the prefix "ft." If $E_{\mathrm{xc}}\left[\rho_{\alpha}(\boldsymbol{r}), \rho_{\beta}(\boldsymbol{r}), \nabla \rho_{\alpha}(\boldsymbol{r}), \nabla \rho_{\beta}(\boldsymbol{r})\right]$ denotes a KS-DFT exchange-correlation functional, in which $\rho_{\sigma}$ is the spin density $(\sigma=\alpha, \beta)$, and $\nabla \rho_{\sigma}$ is the gradient of the density, the translated functional is given by:

$$
E_{\mathrm{ot}}[\rho(\boldsymbol{r}),|\nabla \rho(\boldsymbol{r})|, \Pi(\boldsymbol{r})]=E_{\mathrm{xc}}\left[\rho_{\alpha}^{t}(\boldsymbol{r}), \rho_{\beta}^{t}(\boldsymbol{r}), \nabla \rho_{\alpha}^{t}(\boldsymbol{r}), \nabla \rho_{\beta}^{t}(\boldsymbol{r})\right]
$$


where

$$
\begin{array}{cc}
\rho_{\alpha}^{t}(\boldsymbol{r})=\frac{\rho}{2}(1+f(\rho, \Pi)) & \nabla \rho_{\alpha}^{t}(\boldsymbol{r})=\frac{\nabla \rho}{2}(1+f(\rho, \Pi)) \\
\rho_{\beta}^{t}(\boldsymbol{r})=\frac{\rho}{2}(1-f(\rho, \Pi)) & \nabla \rho_{\beta}^{t}(\boldsymbol{r})=\frac{\nabla \rho}{2}(1-f(\rho, \Pi)) \\
f(\rho, \Pi)=\sqrt{\max \left(0,1-\frac{4 \Pi}{\rho^{2}}\right)}
\end{array}
$$

in which $\nabla \rho$ is the gradient of that spin density. For a single-determinant wave function,

$\frac{4 \Pi}{\rho^{2}} \leq 1$ in all space. However, for a multiconfiguration wave function, this ratio can be greater than one. Notice that the translated functionals depend on $\Pi$ but not on its gradient and that the left and right derivatives of $f$ are not equal at $\frac{4 \Pi}{\rho^{2}}=1$, making the gradient of $f$ discontinuous.

Fully translated functionals also depend on $\nabla \Pi$, and the first and the second derivatives of the functional are continuous. Both kinds of functionals have been found to give reasonably good results for multireference systems.

OpenMolcas supports the use of several different on-top functionals, including a translated local spin-density approximation (LSDA), various translated generalized gradient approximations, and various fully-translated generalized gradient approximations. The following is a list of currently supported functionals: tLSDA,, 54|180|181 $\mathrm{ftLSDA}, \underline{60|180| 181}$

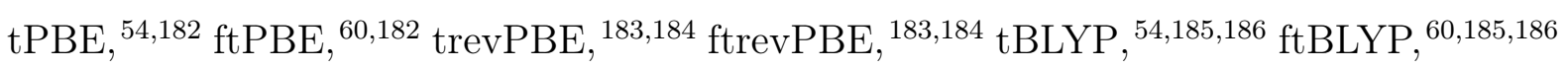
tOPBE, $182|187| 188$ and ftOPBE. $\underline{60 \mid 182] 187 \mid 188}$

OpenMolcas also supports functionals obtained by scaling the exchange and/or correlation components of the exchange-correlation functionals prior to translation. The scaling factors may be input by the user. This allows the functionals to be modified into an HLE-type functional, $\frac{189 / 190}{19}$ where the standard scaling factors are 1.25 for exchange and 0.5 for correlation, but other scaling factors may be chosen.

\subsubsection{Performance of MC-PDFT}

MC-PDFT generally produces results that are comparable in accuracy to complete active space second-order perturbation theory (CASPT2) but at significantly lower computa- 


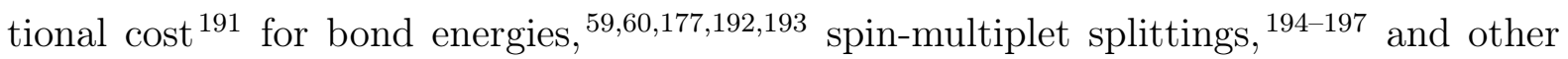
excitation energies. $\frac{198}{201}$ These results are summarized in table 3 . A review containing references for tests and validations on electronically excited states up to mid-2018 is available in ref. 202 .

Table 3: Mean unsigned error (MUE) of MC-PDFT using tPBE functional and CASPT2

\begin{tabular}{|c|c|c|c|}
\hline \multirow[t]{2}{*}{ Reference } & \multirow{2}{*}{$\begin{array}{l}\text { System / } \\
\text { Property }\end{array}$} & \multicolumn{2}{|c|}{ MUE (eV) } \\
\hline & & PT2 & tPBE \\
\hline 54 & $\begin{array}{l}6 \text { covalent or ionic diatomics / } \\
\text { dissociation energy }\end{array}$ & 0.3 & 0.3 \\
\hline 59 & $\begin{array}{l}10 \text { transition metals with ligand / } \\
\text { average bond energy }\end{array}$ & 0.24 & 0.19 \\
\hline 194 & $\begin{array}{l}11 n \text {-acenes (from naphthalene to dodecacene) / } \\
\text { singlet-triplet gap }\end{array}$ & $0.06^{a}$ & $0.13^{a}$ \\
\hline 193 & $\begin{array}{l}13 \text { main-group atoms or compounds / } \\
\text { singlet-triplet gap }\end{array}$ & $0.12^{b}$ & $0.24^{b}$ \\
\hline 196 & $\begin{array}{l}8 \text { organic rings / } \\
\text { singlet-triplet gap }\end{array}$ & $0.01^{b}$ & $0.10^{b}$ \\
\hline 199 & $\begin{array}{l}19 \text { main-group atoms / } \\
\text { first excitation energy }\end{array}$ & 0.22 & 0.42 \\
\hline 201 & $\begin{array}{l}10 \text { doublet radicals / } \\
\text { first } 5 \text { vertical excitation energies }\end{array}$ & 0.14 & 0.19 \\
\hline
\end{tabular}

${ }^{a}$ The reference wave function is optimized from a GASSCF calculation. $57 / 203$

${ }^{b}$ The active space for the reference wave function uses the correlated-participating-orbital (CPO) scheme. 204

\subsubsection{Analytical gradients for SS-CAS-PDFT}

OpenMolcas includes analytical gradients for MC-PDFT when a state-specific CASSCF (SS-CASSCF) wave function is used as a reference (SS-CAS-PDFT), and these gradients enable the fast and efficient determination of equilibrium and transition state structures. ${ }^{205}$ Because MC-PDFT is a nonvariational method, the computation of the gradient requires the construction of a Lagrangian. In general, the computation of analytical gradients can be performed at least an order of magnitude faster than the corresponding gradient calculation using a numerical finite-difference method. SS-CAS-PDFT shows good accuracy compared to experimental data for both equilibrium and transition-state structures. Some illustrative single-processor timings for MC-PDFT calculations employ- 
ing the tPBE on-top functional are shown in table 4

Table 4: Illustrative timings (in seconds) for a single gradient computation ${ }^{a}$ using a single processor.

\begin{tabular}{|c|c|c|c|c|c|c|}
\hline & \multirow[b]{2}{*}{ Active Space } & \multirow[b]{2}{*}{ Symmetry } & \multicolumn{2}{|c|}{ cc-pVDZ } & \multicolumn{2}{|c|}{ cc-pVTZ } \\
\hline & & & CASPT2 & tPBE & CASPT2 & tPBE \\
\hline $\mathrm{NH}_{3}$ & $(6,6)$ & $C_{\mathrm{s}}$ & 16 & 7 & 32 & 14 \\
\hline $\mathrm{HCN}$ & $(8,8)$ & $C_{2 \mathrm{v}}$ & 9 & 4 & 17 & 10 \\
\hline $\mathrm{CH}_{2} \mathrm{O}$ & $(12,9)$ & $C_{2 \mathrm{v}}$ & 29 & 9 & 29 & 15 \\
\hline $\mathrm{HCCH}$ & $(10,10)$ & $D_{2 \mathrm{~h}}$ & 20 & 6 & 20 & 11 \\
\hline oxirane & $(10,10)$ & $C_{2 \mathrm{v}}$ & 55 & 27 & 136 & 45 \\
\hline pyrrole & $(6,5)$ & $C_{2 \mathrm{v}}$ & 87 & 39 & 1082 & 212 \\
\hline acrolein & $(4,4)$ & $C_{\mathrm{s}}$ & 558 & 117 & 3390 & 160 \\
\hline butadiene & $(4,4)$ & $C_{2 \mathrm{~h}}$ & 85 & 29 & 933 & 137 \\
\hline pyridine & $(6,6)$ & $C_{2 \mathrm{v}}$ & 183 & 32 & 2256 & 156 \\
\hline maleic anhydride & $(8,7)$ & $C_{2 \mathrm{v}}$ & 123 & 36 & 1148 & 218 \\
\hline
\end{tabular}

${ }^{a}$ CASPT2: Numerical gradient. tPBE: Analytical MC-PDFT gradient.

\subsubsection{Density matrix renormalization group pair density functional theory (DMRG-PDFT)}

DMRG (see section 3.2 is a powerful method to treat static correlation, especially in molecular systems requiring large active-spaces. MC-PDFT can be used in conjunction with DMRG to add additional dynamic correlation in an inexpensive way. The first applications $^{206 \mid 207}$ of DMRG-PDFT were based on an interface between the existing MCPDFT code in OpenMolcas and the DMRG code in the QCMAQUIS ${ }^{117126 \mid 208}$ program. An illustrative example ${ }^{206}$ of singlet-triplet gaps in polyacenes is shown in fig. 6. The tPBE $/ 6-31 G+(d, p)$ combination of on-top functional and basis set was used.

\subsubsection{State-interaction pair-density functional theory}

The accurate description of ground- and excited-state PESs in cases where two electronic states are strongly interacting (such as near conical intersections or locally avoided crossings) is a challenge for many electronic structure methods. A new framework, called state-interaction PDFT (SI-PDFT),$\sqrt[209]{ }$ has been introduced to handle these situations in 


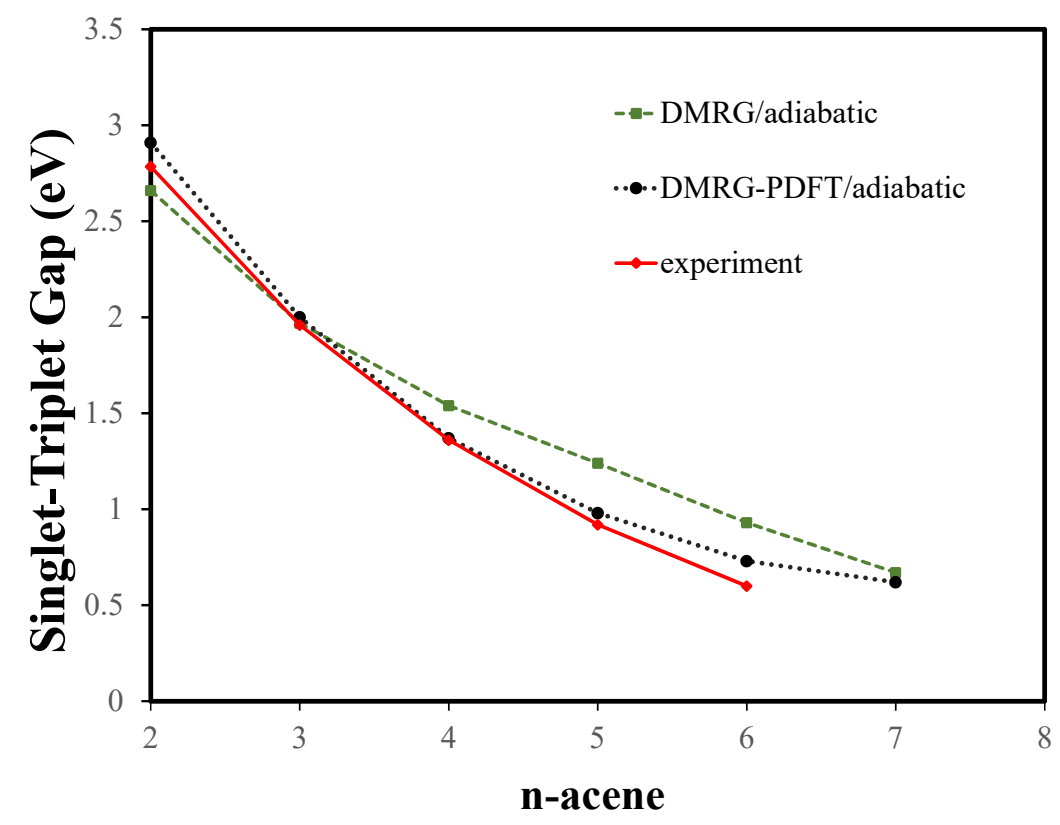

Figure 6: Singlet-triplet gaps in polyacenes.

the case of MC-PDFT. This has been recently implemented in OpenMolcas and successfully applied to several cases, such as the dissociation of $\mathrm{LiF}^{209}$ and the charge-transfer reaction path of a mixed-valence compound ${ }^{210}$ SI-PDFT is an extension of MC-PDFT that involves the diagonalization of an $N \times N$ effective Hamiltonian to generate a new set of $N$ electronic states with proper PES topology in regions of strong state interaction. This method is similar in objectives and application to the "perturb-then-diagonalize" approaches in multireference perturbation theories such as MS-CASPT2 211212 or MCQDPT. 2131214 An illustrative example is shown in fig. 7. The tPBE functional was used for both a standard MC-PDFT calculation and a SI-PDFT calculation. While unphysical curve crossings are present in the standard MC-PDFT treatment, these vanish when the SI-PDFT methodology is used because the final step in the SI-PDFT method is a diagonalization. 


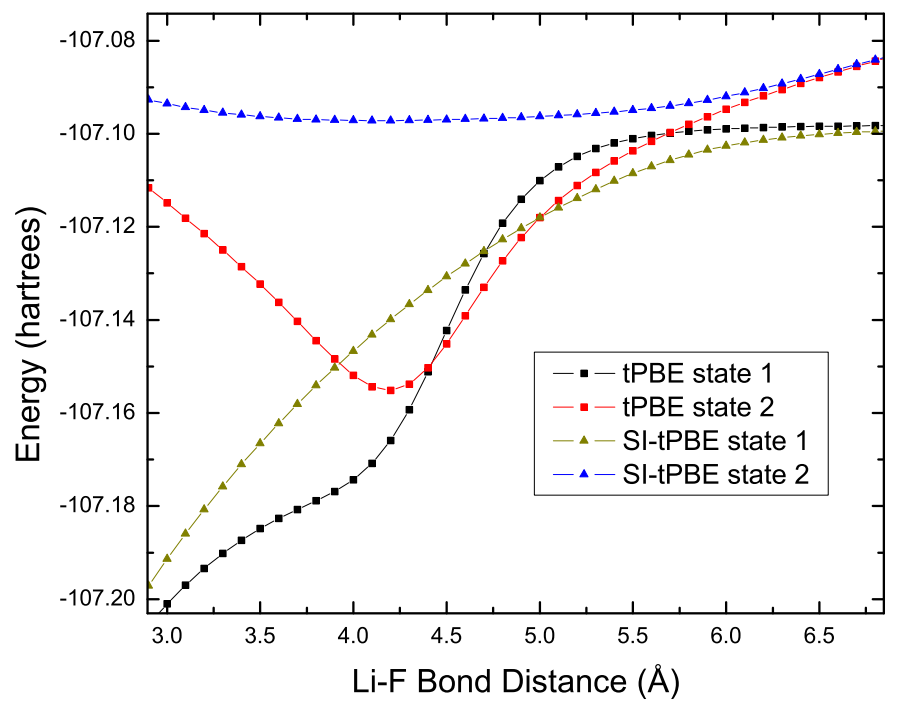

Figure 7: Dissociation of LiF calculated with a $(6 \mathrm{e}, 6 \mathrm{o})$ active space.

\section{Exploration of potential energy surfaces}

Understanding the mechanism, rate and yield of chemical reactions requires exploring the potential energy surfaces of interest. A photochemical reaction involves by definition several PESs; the strong coupling between the different electronic states promotes efficient nonradiative electronic transitions. ${ }^{2151216}$ One distinguishes between internal conversion caused by nonadiabatic coupling between states of same spin multiplicity, and intersystem crossing caused by spin-orbit coupling between states of different spin multiplicity. Exploration of PESs can be performed either through optimization of specific geometries and paths along the reaction, or through simulations of the actual dynamics of the reaction in time.

Regarding optimization calculations, OpenMolcas allows one to optimize minima and transition states, as well as minimum-energy paths and intrinsic reaction paths, using the SLAPAF program. Since recently, it is also possible to optimize conical intersections thanks to the implementation of the nonadiabatic coupling vector. This new feature is detailed in section 4.1. We mention in passing that the SHARC suite (described in section 4.3 provides a module, alternative to the SLAPAF program in OpenMolcas, that allows the optimization of excited-state minima, minimum-energy crossing points, and conical 
intersections, using OpenMolcas for calculating the needed electronic quantities.

Regarding dynamics simulations, direct methods calculate the PESs as needed along trajectories, sampling only the relevant regions of the PESs. These nuclear trajectories are then used to describe the nuclear wavepacket motion. One major feature that differentiates the several direct methods able to describe (nonadiabatic) dynamics is the treatment of the nuclear motion through the basis of nuclear trajectories. ${ }^{217}$ OpenMolcas allows one to simulate Born-Oppenheimer and surface-hopping dynamics, by treating the nuclei classically. New features presented in section 4.2 include thermal sampling of the initial conditions, application of external mechanical forces and study of isotopic effects. As an electronic structure package, OpenMolcas is also interfaced to other open-source dynamics codes. The interfaces to SHARC and Quantics are presented in sections 4.3 and 4.4 respectively. SHARC contains a general surface-hopping algorithm with arbitrary couplings such as the spin-orbit coupling, while Quantics allows a quantum treatment of the nuclear motion with the direct dynamics variational multiconfiguration Gaussian (DD-vMCG) method.

\subsection{Conical intersection optimization and characterization}

A key quantity for describing photochemical processes and near-degeneracy between electronic states is the nonadiabatic or derivative coupling vector. However, until now it was not possible to obtain this quantity with the MOLCAS package, and some approximations had to be employed for nonadiabatic molecular dynamics ${ }^{8}$ or for the location of conical intersections. $\frac{218}{21}$

Since its release, OpenMolcas includes analytical nonadiabatic couplings between state-average CASSCF wave functions, as described in ref. 219. The implementation is based on the algorithm used for energy gradients,,$\frac{146|148| 220}{12}$ and it is compatible with both conventional and density-fitted two-electron integrals. The availability of nonadiatabic couplings allows the use of the projected constrained optimization (PCO) method 2211222 for locating minimum-energy conical intersections (MECI). From the user's perspective, the only change with respect to a single-surface geometry optimization is the addition 
of an energy difference constraint - gradients and nonadiabatic couplings are computed automatically as needed. Furthermore, the first-order conical intersection characterization proposed in ref. 219 is also reported upon convergence, allowing the unambiguous description and comparison of conical intersections topographies. The meaning of the characterization parameters is illustrated in fig. 8. From these, it is possible to determine whether the intersection is peaked or sloped - in the latter case, it is not a minimum on the upper surface - and this is available in the output as well.

(a)

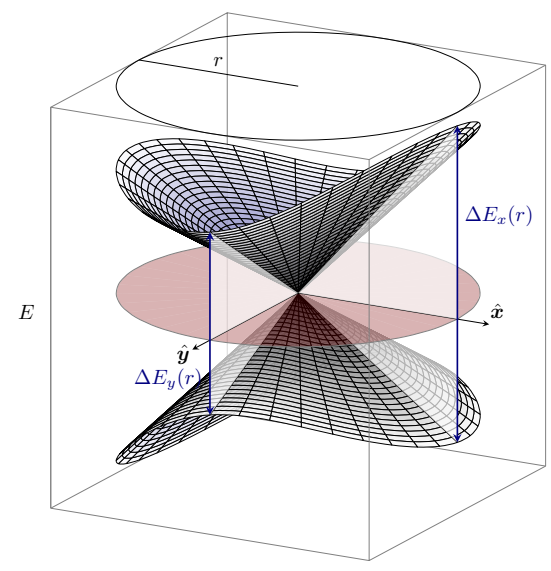

(b)

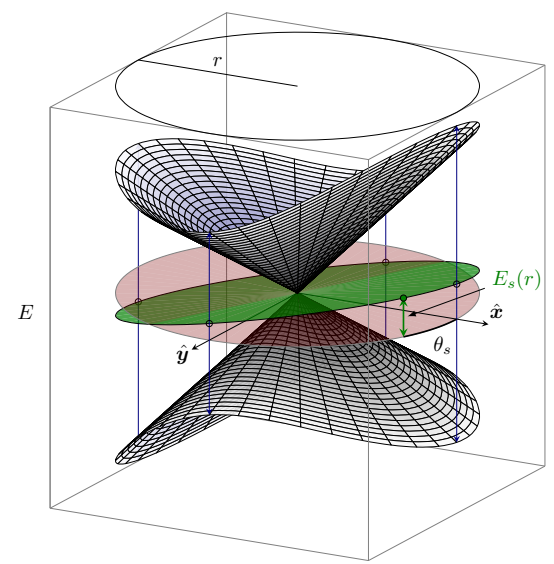

$\delta_{g h}=\sqrt{\frac{\Delta E_{x}(r)^{2}+\Delta E_{y}(r)^{2}}{8 r^{2}}}$

Figure 8: Illustration of the parameters characterizing a conical intersection. The red disc represents the horizontal plane $E=E^{\times}$. (a) Energy differences in the $\hat{\boldsymbol{x}}$ and $\hat{\boldsymbol{y}}$ directions define the pitch $\delta_{g h}$ and asymmetry $\Delta_{g h}$. (b) The green disc represents the average energy plane, as indicated by the small circles on its edge, the amount and orientation of its maximum deviation from the red disc define the relative tilt $\sigma$ and the tilt heading $\theta_{s}$.

\subsection{Dynamics simulations within OpenMolcas}

Born-Oppenheimer and nonadiabatic dynamics can be performed within OpenMolcas using the DYNAMIX and SURFACEHOP modules. Transitions between different electronic states can be treated by two different approaches: a) According to the quantification of the temporal rate of mixing between electronic states, $\stackrel{223}{23}$ and b) following Tully's fewest switches approach ${ }^{224}$ where the initial population density matrix is defined at the beginning of the propagation. The modular structure of OpenMolcas allows combining other features in OpenMolcas with dynamics simulations. For example, the ESPF mod- 
ule enables hybrid quantum mechanics/molecular mechanics (QM/MM) simulations or any other electronic structure method available in OpenMolcas can be used to obtain the energy and gradients. Recent developments for molecular dynamics simulations have been included in OpenMolcas. They are summarized below.

\subsubsection{Initial thermal sampling}

In order to obtain statistically significant predictions using molecular dynamics simulations, an ensemble of trajectories has to be computed (usually hundreds of trajectories, even in simple reactions like trans-cis photoisomerization $225 \sqrt{230})$. The initial state of the system has to be appropriately sampled in order to get a good representation of nuclear position and momenta. OpenMolcas allows sampling of the phase-space for a thermally equilibrated state according to the Boltzmann distribution. Given the minimum energy structure of the system, the absolute temperature, and a second-order expansion of the potential energy as determined with a frequency calculation, initial conditions (i.e. nuclear coordinates and momenta) are generated; they can be subsequently used for running individual trajectories.

\subsubsection{Steered molecular dynamics}

DYNAMIX has the possibility of including multiple external force pairs, each one applied on a specific couple of nuclei. The external forces are added to the molecular force field defined by the electronic state. This new feature allows the user to simulate the action of external forces (i.e. mechanical effects) acting over a molecular system. The magnitude of each force pair is defined as the magnitude of each force vector acting on each of the two atoms. Many force pairs can be added, generating a final external force vector by summing up all the components on each atom. Finally, the applied force pairs can be defined as extension or compression forces. In this way different mechanochemical setups (e.g. polymer mechanochemistry, sonochemistry or force probes) can be simulated, in a first approach, by adding external forces.

Steered molecular dynamics can be performed in ground and excited states, permitting 
the study of photochemical processes in strained systems. Photochemistry of mechanically affected systems, i.e. mechano-photochemistry, still remains quite unexplored, and only few studies show the interesting properties of these processes. In this regard, it has been shown that photochemistry can be effectively modified by mechanical means, 231 where the interplay between excited-state dynamics and external forces is far from being simple and intuitive, as is the case of a trans to cis photoisomerization of a retinal model system, where an extension force, expected to oppose the formation of cis isomer, is found to enhance the photoisomerization, significantly increasing the photoreaction quantum yield. 229

\subsubsection{Isotope effect}

DYNAMIX is making use of the isotope feature in OpenMolcas which enables the consideration of specific isotopes in molecular dynamics simulations. Typically, replacing an atom with an isotope is associated with a kinetic isotope effect which affects the reaction rate of a chemical process.

In a recent study of the photoisomerization in rhodopsin, the new feature helped to reveal a new type of kinetic isotope effect. ${ }^{232}$ In this specific case, the hydrogen atoms of the 11-cis isomerizing double bond were replaced by deuterium in three different ways: at both hydrogens, and at the hydrogen at either the 11 or 12 position (fig. 9). The molecular dynamics simulations together with time-resolved measurements showed that while the reaction rate was mainly unaffected, the isomerization quantum yield was significantly altered by the deuterium replacements. In fact, remarkably, the quantum yield was found to decrease and increase in the monodeuterated and bideuterated cases, respectively. This study provided further evidence on the importance of the "hydrogen out of plane" mode for the description of the reaction coordinate of the photoisomerization of the 11-cisretinal protonated Schiff base chromophore of vertebrate visual rhodopsins. $\frac{2331234}{21}$

Changing the masses of atoms to "artifical" values may also be a useful simulation tool to get insights into the factors that determine the yield of chemical reactions. In a recent study of the dissociation of dioxetane compounds, this functionality was used 


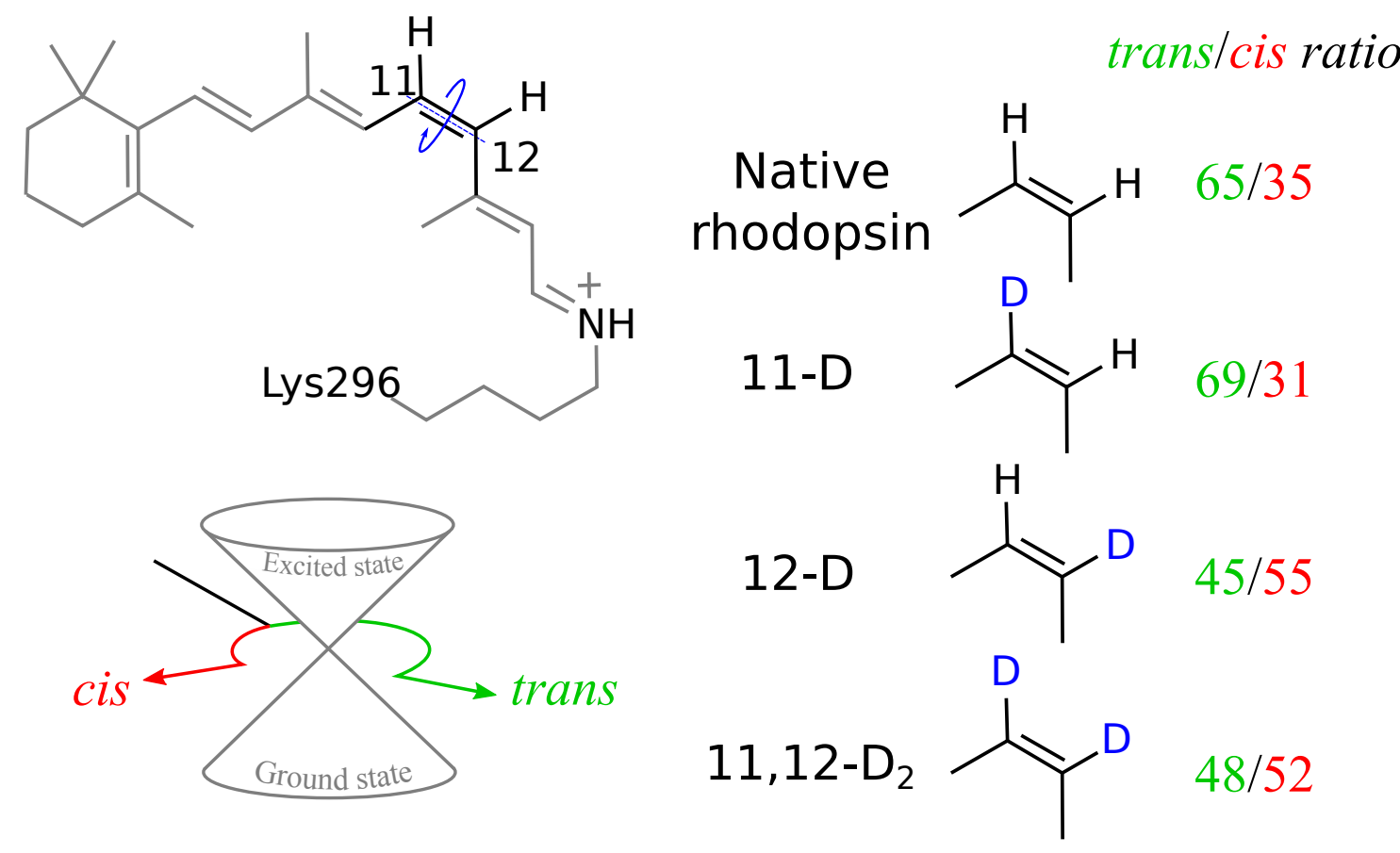

Figure 9: 11-cis-Retinal protonated Schiff base and the three studied isotopomers.

to understand the effect of methylation. ${ }^{235}$ Substitution of hydrogen atoms by methyl groups in dioxetane had been shown to lead to a longer dissociation time and it had been previously suggested that this was due to the increase in the number of degrees of freedom. To investigate further the effect of methylation, dynamics was simulated for the unmethylated 1,2-dioxetane, but where the mass of the four hydrogen atoms was increased to reproduce the moment of inertia of the methyl groups. The simulations suggest that approximately $75 \%$ of the increase in the dissociation time is actually due to a pure mass effect (in contrast to the hypothesis put forward in a previous theoretical study).

\subsection{Nonadiabatic semiclassical dynamics interface}

OpenMolcas is interfaced with the ab initio molecular dynamics software suite SHARC (Surface Hopping including ARbitrary Couplings). 236 240 Through this interface, nonadiabatic dynamics can be carried out based on the CASSCF and CASPT2 electronic structure methods of OpenMolcas.

SHARC is a generalization of the surface hopping method. $\frac{224}{}$ This allows describing processes such as intersystem crossing or laser-induced transitions, which is not possible 
with standard Tully surface hopping. Furthermore, the SHARC software suite can manage large trajectory ensembles and features many analysis tools.

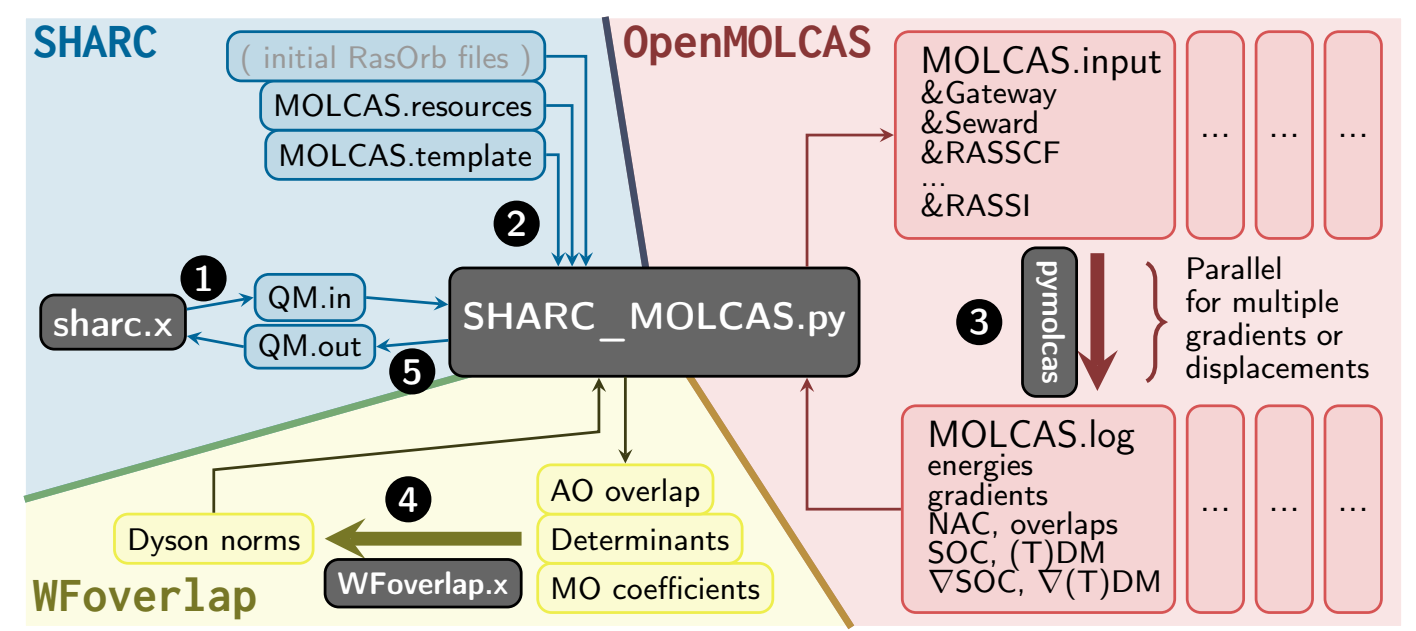

Figure 10: Flow chart of the SHARC-OpenMolcas interface. In step (1), the SHARC dynamics driver sharc. $\mathrm{x}$ writes a file with the current geometry and requests for quantities (e.g., energies $E$, spin-orbit couplings (SOC)). This file is read by the interface, together with the template and resource files that define the level of theory and the computational parameters, respectively. In step (2), all necessary OpenMolcas input files are written and step (3) executes the pymolcas driver. The resulting output files are parsed for all requested quantities. Optionally (4), the WFOVERLAP is executed to obtain Dyson norms. Finally (5), all obtained results are handed back to the SHARC driver.

The interface between SHARC and OpenMolcas is based on file communication between the two programs, see the flow chart in fig. 10. The SHARC dynamics driver (the master process) writes a request file at each simulation time step, containing the current geometry and a list of required electronic quantities, such as energies, gradients, spin-orbit couplings, etc. The interface, a Python script, combines the request information with the electronic structure settings and computational parameters. The interface writes the OpenMolcas input files, allowing to compute multiple gradients or displacements for numerical gradients in a task parallel fashion. The automatically generated OpenMolcas input employs the GATEWAY and SEWARD modules for setup and integrals, the RASSCF module for wave functions and energies, RASSI for transition dipole moments, spin-orbit couplings, and wave function overlaps (for nonadiabatic interactions, see below), and MCLR and ALASKA for gradients. For CASPT2 or MS-CASPT2, the calculation of numerical gradients is directly controlled by the SHARC-OpenMolcas interface, allowing a simultaneous calculation of all gradients of all states and the derivatives 
of dipole moments and spin-orbit couplings in a single loop. The nonadiabatic interaction between the different states is described by employing wave function overlaps ${ }^{241}$ from the RASSI module, or by nonadiabatic coupling vectors from MCLR/ALASKA. QM/MM dynamics (CASSCF/force fields) can also be performed through the OpenMolcas-Tinker interface. All collected electronic structure data is finally communicated back through the interface to the dynamics driver, which propagates the trajectory to the next time step.

It is noted that the SHARC-OpenMolcas interface can also produce Dyson norms to approximately describe ionization probabilities (see also section 5.3 by feeding the relevant wave function data into the WFOVERLAP program ${ }^{242}$ within the SHARC suite.

\subsection{Quantum dynamics interface}

While implementing a direct dynamics code for classical nuclei is rather straightforward since information is only needed at a given position in space, a quantum description of the nuclei has more problems. We report here a new interface between OpenMolcas and the Quantics package ${ }^{243}$ (grown out of the Heidelberg MCTDH package ${ }^{244}$ ), which allows a quantum mechanical treatment of both electronic and nuclear dynamics via the direct dynamics variational multiconfiguration Gaussian (DD-vMCG) method. $2172245 \mid 246$ In practice, Quantics uses OpenMolcas to build a local harmonic approximation of the coupled PESs, as represented in fig. 11. The dynamics can be run either in Cartesian coordinates or in normal modes. If the latter, transformation matrices between the two systems of coordinates are set up by reading the output file of a frequency calculation (using the MCKINLEY module with OpenMolcas).

Along the dynamics simulation, Quantics writes input files from a template input file to run OpenMolcas and compute the adiabatic energies, gradients, Hessians and couplings for all electronic states involved. The electronic structure calculations are performed at the centre point of each Gaussian basis function and subroutines in Quantics parse the OpenMolcas output files to extract the relevant information. ${ }^{247}$ Currently possible electronic structures methods are $\mathrm{HF}, \mathrm{CASSCF}$ and CASPT2. In the latter case, it is only 


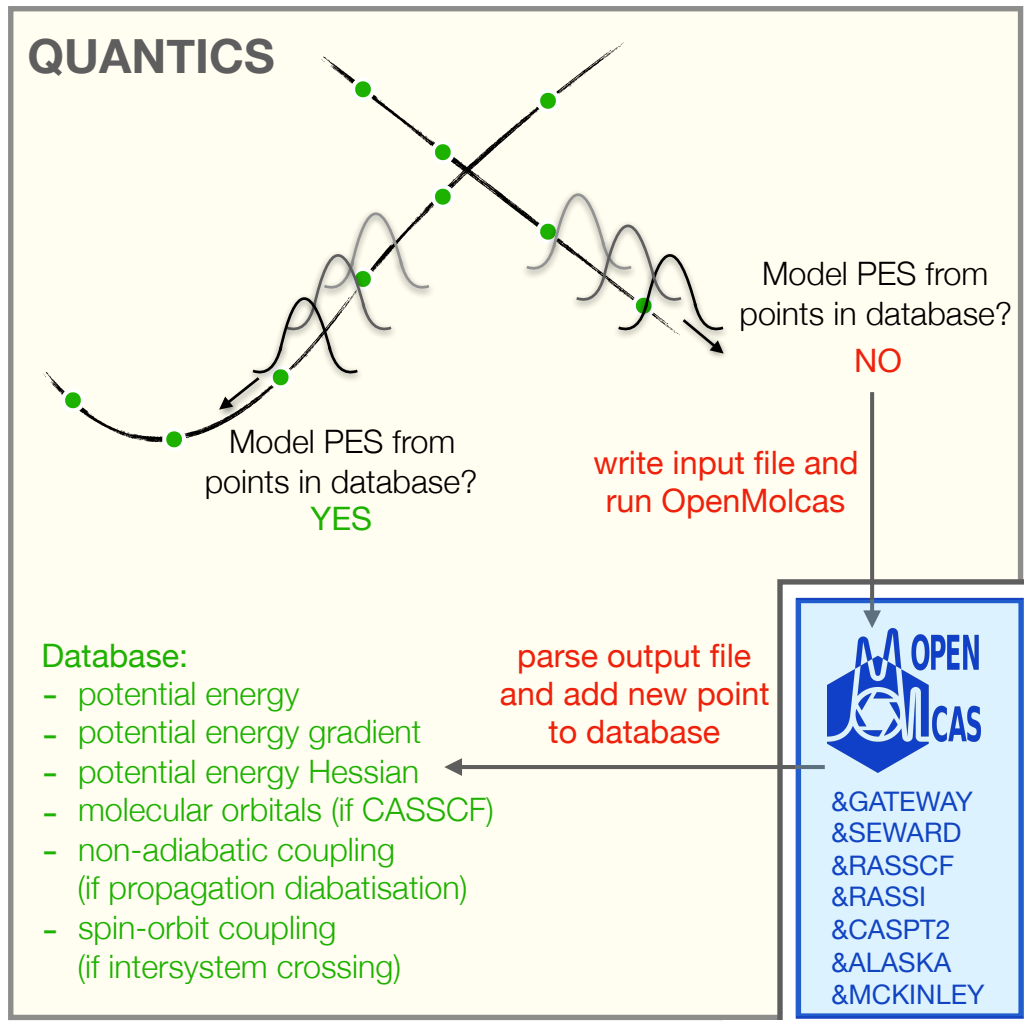

Figure 11: Quantic-OpenMolcas interface. At each timestep, the database of previously run quantum chemistry calculations is probed to build model coupled potential energy surfaces (PESs). If suitable points exist around the desired geometry (Gaussian basis function on the left hand side of the figure), a representation of the PES is constructed using those points. If that region of the coordinate space has not been previously explored (right hand side), an OpenMolcas calculation is performed, and information about this new point is added to the database. 
the CASPT2 potential energies that are used while the corresponding energy derivatives and couplings are approximated with their values at CASSCF level.

If several electronic states are included in the simulation, the dynamics is performed in a diabatic basis in order to avoid singularity issues. A diabatization operation is then realised to transform the adiabatic quantities provided by OpenMolcas into diabatic ones. Two diabatization schemes are available: the regularisation diabatization method, $\stackrel{248}{2}$ which limits the application to two electronic states, and the propagation diabatization method implemented more recently. ${ }^{249}$ The former method requires information about a reference conical intersection geometry (which can be optimised using the SLAPAF module with OpenMolcas). The latter method requires the calculation of the nonadiabatic coupling vector (which can be done using the ALASKA module with OpenMolcas) at every new electronic structure point. When dealing with states of different spin multiplicity, a diabatization is performed in each spin-multiplicity subset, and internal conversions within each subset is accounted for as usual in same-spin dynamics. Intersystem crossing is treated by calculating the spin-orbit coupling terms (which can be done using the RASSI module in OpenMolcas) at every new electronic structure point. $\underline{163}$

In order to save time and avoid redundant electronic structure calculations, the electronic quantities calculated by OpenMolcas are stored in a database. New database points are only calculated if the new nuclear geometry differs by some predetermined amount from all database geometries (right basis function in fig. 11). If it is close enough to some database points (left basis function in fig. 11), the quantities are interpolated from the values present in the database. $\frac{250}{2 t}$ is noted that the molecular orbital coefficients of the CASSCF calculations are also stored in the database and used as initial guesses for new electronic structure calculations. Also, instead of calculating the Hessian at every point, after the first point, an update procedure can be performed using the database according to the Powell method. ${ }^{246[251}$ By running the dynamics calculation several times, more and more points are added to the database. The database "grows" iteratively and reaches convergence once no new database points are needed. 


\section{$5 \quad$ Spectroscopic and magnetic properties}

The strong focus of the OpenMolcas package in multiconfigurational wave function methods makes it an excellent tool for the computational study of systems where multiple electronic states play an important role. An obvious case is the study of electronic spectra, involving transitions between different electronic states. A paradigmatic example is the study of transition metal complexes, where the presence of partially filled $d$ and/or $f$ shells gives rise to many close-lying electronic states of various spin multiplicities.

The workhorse for these investigations, once accurate spin-adapted wave functions and energies are obtained, is the RASSI program. $\stackrel{163}{ }$ This program can compute the spin-orbit interaction to yield a set of spin-orbit eigenstates, with the use of atomic mean-field integrals, $\stackrel{252}{2}$ as well as transition properties between different wave functions.

In this section we report some recent developments and improvements for the computation of spectroscopic and magnetic properties in OpenMolcas, from the exact semiclassical treatment of light-matter interaction (section 5.1) to applications in X-ray spectroscopy (section 5.2), photoelectron spectroscopy (section 5.3), magnetic circular dichroism (section 5.4), molecular magnetism (section 5.5), and in the context of ultra-accurate calculations (section 5.6.).

\subsection{Light-matter interaction and beyond the multipole expan- sion}

All spectroscopies depend on the interaction between the system and an external electromagnetic field. For weak fields that can be treated as perturbations, the oscillator strength for a transition from an initial state $i$ to a final state $f$ is:

$$
f_{i f}(\omega) \propto|\langle i|\hat{U}| f\rangle|^{2} \delta\left(\omega-\omega_{i f}\right)
$$

where $\omega_{i f}=\left(E_{f}-E_{i}\right) / \hbar$ is the resonance angular frequency and $\hat{U}$ is the time-independent part of the electromagnetic perturbation. Usually, the field is described by a plane wave 
and using only terms linear in field strength $\hat{U}$ then becomes:

$$
\hat{U} \propto \sum_{j}\left[\exp \left(\mathrm{i} \boldsymbol{k} \cdot \boldsymbol{r}_{j}\right)\left(\boldsymbol{\varepsilon} \cdot \hat{\boldsymbol{p}}_{j}\right)+\mathrm{i} \frac{g}{2} \exp \left(\mathrm{i} \boldsymbol{k} \cdot \boldsymbol{r}_{j}\right)(\boldsymbol{k} \times \boldsymbol{\varepsilon}) \cdot \hat{\boldsymbol{s}}_{j}\right]+\text { c.c. },
$$

where the sum goes over all the electrons, $\boldsymbol{k}$ is the wave vector, $\boldsymbol{r}_{j}$ is the position, $\boldsymbol{\varepsilon}$ is the polarization vector, $\hat{\boldsymbol{p}}_{j}$ the momentum operator, $g$ is the electronic spin $g$-factor, and $\hat{\boldsymbol{s}}_{j}$ the spin operator, and c.c. the complex conjugate of the previous terms.

Traditionally, the integrals in eq. (12) are evaluated using a multipole expansion of the electromagnetic wave:

$$
\exp \left(\mathrm{i} \boldsymbol{k} \cdot \boldsymbol{r}_{j}\right)=1+\mathrm{i}\left(\boldsymbol{k} \cdot \boldsymbol{r}_{j}\right)-\frac{1}{2}\left(\boldsymbol{k} \cdot \boldsymbol{r}_{j}\right)^{2}+\ldots
$$

For the transition moments, taking only the zeroth-order term amounts to the electric dipole approximation, while the first-order term is associated with electric quadrupole and magnetic dipole interactions. The multipole expansion leads to well-recognized selection rules that for certain systems and specific choices of coordinate systems can be directly related to electronic structure. While the dipole approximation is often sufficient for the low-energy photons in the optical region, high-energy photons have large $\boldsymbol{k}$ vectors, rapidly oscillating fields, and higher-order terms in the multipole expansion cannot be ignored. These higher-order terms depend on the choice of origin, at least in cases where there are nonzero terms of lower order. For weak fields the problem of origin dependence was recently solved by Bernadotte et al., by including all terms to the same order in the oscillator strength and not in the transition moments, which for the second order requires calculations up to magnetic quadrupoles and electric octupoles. $\frac{253}{2}$ The complete second-order expansion is implemented in OpenMolcas and has been used to calculate

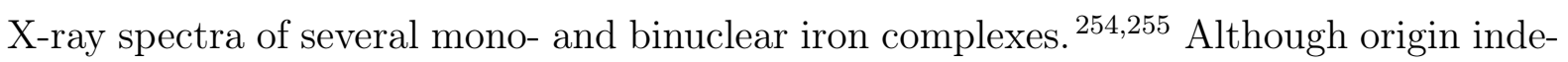
pendence was first proven for the velocity gauge, $\frac{253}{25}$ origin independence in a finite basis set can also be accomplished in the length gauge. $\frac{256}{2}$ However, what is usually referred to as the length gauge is actually a mixed gauge, with the electric components in the length gauge and magnetic components in the velocity gauge. $\frac{256}{25}$ Origin independence, in finite 
basis sets, is not conserved in this mixed gauge. $\frac{257}{2}$ Furthermore, when using the complete second-order expansion the increased basis set requirements for higher-order terms in the multipole expansion must be considered. 257

A solution to the problems with the multipole expansion is to instead use the planewave form of the wave vector directly: the exact semiclassical light-matter interaction 258 is origin independent, cannot give negative oscillator strengths and gives what all terms in the multipole expansion ideally should converge to. $\frac{258}{25}$ Further, it shows better numerical stability with respect to the choice of basis set. $\frac{259}{25}$ OpenMolcas now includes an elegant and efficient procedure using a standard Gauß-Hermite quadrature to evaluate the integrals in eq. 12 in this formalism. $\frac{259}{}$ Both electric and magnetic terms are calculated this way, with the spin-magnetic term in eq. 13 being nonzero when the spin-orbit operator in the RASSI module is used.

With the exact operator OpenMolcas now calculates the angular dependence on the transition intensities with respect to both polarization and wave propagation direction. This can be used to simulate experiments where the system is oriented relative to the photon beam, e.g., in single crystals or molecules on surfaces. Although no closed formula for the isotropically tensor averaged oscillator strengths is known, the exact value can be approximated by averaging over different directions using a Lebedev grid. $\frac{259 \mid 260}{}$ Although the averaging over a grid increases the computational time compared to the multipole expansion, this will only rarely affect the timing of the full electronic structure calculation. To illustrate the capabilities of the exact operator, the angular dependence of the single $\mathrm{Cu} 1 \mathrm{~s} \rightarrow 3 \mathrm{~d}$ transition in single-crystal $\left[\mathrm{CuCl}_{4}\right]^{2-}$ has been calculated and compared to the experimental values, see fig. 12, ${ }^{259}$ The experimental intensity is distinguished by a four-fold periodicity, which was used to assign the electric quadrupole contributions. $\frac{261}{26}$ Further, the orientation of the peak maxima can also be used to identify the symmetry of the singly-occupied $3 \mathrm{~d}$ orbital. Calculations with the exact operator show the same angular dependence, including the four-fold periodicity, which illustrates how the exact operator includes any information that traditionally have been assigned to multipole contributions. 


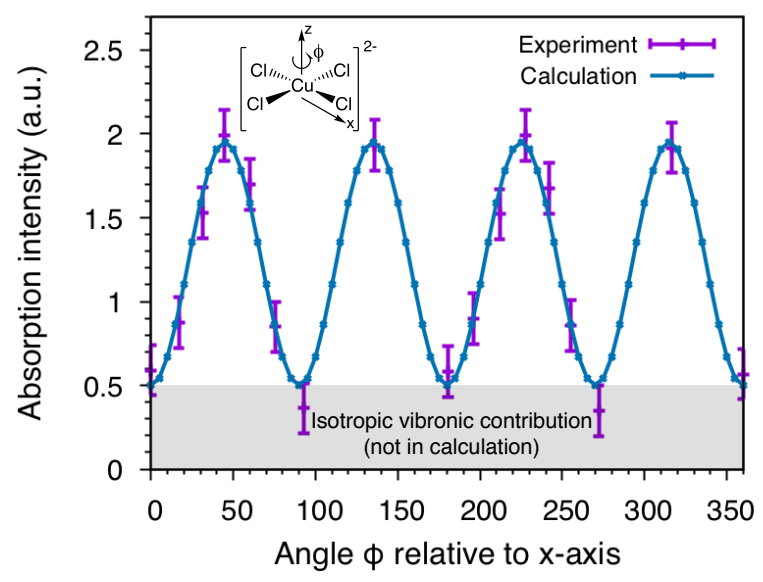

Figure 12: Angular dependence of the $1 \mathrm{~s} \rightarrow 3 \mathrm{~d}$ copper $\mathrm{K}$ pre-edge transition in $\left[\mathrm{CuCl}_{4}\right]^{2-}$. Experimental data are normalized K pre-edge peak heights. ${ }^{261}$ Calculated values are from the exact semiclassical light-matter interaction with intensity uniformly scaled to match experiment. $\frac{259}{25}$ The isotropic contributions are due to vibronic coupling, which are not included in the calculations.

\subsection{X-ray spectroscopy and calculations of many core-hole ex- cited states}

$\mathrm{X}$-ray spectroscopy involves core-hole states that are at least hundreds of eV higher in energy than the valence excited states. In a complete-active-space approach, these states can be included in the CI expansion by placing the relevant core orbitals in the active space. As the number of excitations from these orbitals can be restricted to one, it is convenient to use a restricted active space (RAS) wave function to simulate X-ray spectra. $\frac{262 \sqrt{265}}{26}$ For such processes, the usual bottom-up approach that includes all states is problematic because to reach core-hole excited states all possible valence excited configurations must be calculated first. A few strategies for generating core-hole states are available for example, for some systems where it is possible to use symmetry considerations, or for very small active spaces. $\frac{266}{26}$ However, for a standard-size active space there can be millions of valence states. Strategies that only reach core-hole states are needed. Two of them recently implemented in OpenMolcas are reported here.

A convenient solution to this problem is to define a fixed orbital function $\chi$ and a 
parameter $\lambda$, and add a term to the molecular Hamiltonian

$$
\hat{H}_{\mathrm{sh}}=\hat{H}+\lambda \hat{P}[\chi]
$$

Here, $\hat{H}_{\mathrm{sh}}$ is the shifted Hamiltonian, $\hat{P}[\chi]$ is an orthogonal projection on the space of wave functions where orbital $\chi$ is doubly occupied. This is multiplied by $\lambda$ and can be implemented by modifying the two-electron MO integrals at the CI step. These integrals are recomputed at each macroiteration while the added term is using the same fixed function $\chi$ all the time. The penalty term has the effect of raising the energy of those configurations with a filled core hole to be above those with a single hole, which are now the lowest in energy. Our experience shows that it is easy to find a suitable shift $\lambda$, such that this scheme is stable across changing orbitals and structure, and it has been observed to give only a slight energy variation with the input shift parameter. Obviously, special basis functions may have to be supplied to allow the orbitals of the core-hole states to relax accurately, or if inner-valence correlation is important, e.g. for Auger. The following example shows the calculation of core-valence excitations for $\mathrm{H}_{2} \mathrm{O}$ close to equilibrium, using a specifically prepared basis set. Two calculations were performed: one without the projected shift, and the other with the shift. The orbital energies in table 5 show large differences between without and with the core hole present. The results of the subsequent RASSI calculation are displayed in table 6, showing that it is possible to access states very high in energy (compared with typical valence excitations). For other examples, please see the on-line poster in ref. 267.

Table 5: Orbitals used for $\mathrm{H}_{2} \mathrm{O}$. Starred are valence/Rydberg orbitals, unoccupied in the ground state. Orbital energies $\left(E_{\mathrm{h}}\right)$, for the ground state, and for the excited states (average, hence approximate).

\begin{tabular}{lccccccccc} 
& $1 a^{\prime}$ & \multicolumn{1}{c}{$2 a^{\prime}$} & \multicolumn{1}{c}{$3 a^{\prime}$} & \multicolumn{1}{c}{$4 a^{\prime}$} & \multicolumn{1}{c}{$5 a^{\prime *}$} & \multicolumn{1}{c}{$6 a^{\prime *}$} & $7 a^{\prime *}$ & $1 a^{\prime \prime}$ & $2 a^{\prime \prime *}$ \\
\hline ground & -20.6 & -1.36 & -0.72 & -0.59 & 0.03 & 0.03 & 0.04 & -0.51 & 0.04 \\
excited & -21.3 & -1.78 & -1.13 & -1.02 & -0.07 & -0.01 & 0.01 & -0.97 & 0.01 \\
\hline
\end{tabular}

For the general case, another simple technique is to remove from the CI all configurations with fully occupied core orbitals, the so-called core-valence separation (CVS). $\frac{268}{6}$ 
Table 6: States, excitation energies (eV), dipole and velocity Einstein $A$ coefficients $\left(\mathrm{ps}^{-1}\right)$, and polarization $\left(C_{s}\right)$.

\begin{tabular}{lcccc} 
Excitation $(\mathrm{RASS})$ & Energy & Dipole & Velocity & Polarization \\
\hline $5 a^{\prime} \leftarrow 1 a^{\prime}(\mathrm{O} 1 \mathrm{~s})$ & 537.2 & 0.124 & 0.139 & $A^{\prime}$ \\
$6 a^{\prime} \leftarrow 1 a^{\prime}(\mathrm{O} 1 \mathrm{~s})$ & 539.1 & 0.316 & 0.289 & $A^{\prime}$ \\
$7 a^{\prime} \leftarrow 1 a^{\prime}(\mathrm{O} 1 \mathrm{~s})$ & 539.8 & 0.065 & 0.052 & $A^{\prime}$ \\
$2 a^{\prime \prime} \leftarrow 1 a^{\prime}(\mathrm{O} 1 \mathrm{~s})$ & 537.7 & 0.075 & 0.065 & $A^{\prime \prime}$ \\
\hline
\end{tabular}

Because of the large energy separation between configurations with and without core holes, the latter do not contribute significantly to the core-hole excited states. For activespace methods the CVS is closely related to the generalized active space method. $\frac{57}{\text { Using }}$ the special case of RAS, the core orbitals can be placed in RAS1 allowing at least singles, for single-core-hole excited states, or doubles, for double-core-hole excited states. The core-hole excited states are found by applying a core-hole $(\mathrm{ch})$ projection operator $P_{\mathrm{ch}}$

$$
P_{\mathrm{ch}} \Psi=\Psi_{\mathrm{ch}}
$$

on the RASSCF calculation, which for single-core-hole excited states removes all configurations in $\Psi$ that are fully occupied in RAS1. 254269 This ensures that the lowest-energy state in $\Psi_{\mathrm{ch}}$ is a core-hole excited state in the RASSCF optimization. It is noted that although the core-hole and valence states are calculated separately and are thus not orthogonal, transition intensities can still be calculated in the RASSI module ${ }^{1631270}$ since the different wave functions use the same CI expansion.

In X-ray spectroscopy, even if the computation of all valence states can be avoided using one of the two strategies discussed above, the number of excited states needed to describe the process of interest can be very large. In transition metal complexes, the density of states is very high so that, for an X-ray absorption spectrum that typically spans $10 \mathrm{eV}$, several hundred final states are required. ${ }^{266}$ This creates difficulties for standard techniques such as a multi-root Davidson ${ }^{[77}$ or Davidson-Olsen algorithm. ${ }^{271}$ A modified CI algorithm has therefore been implemented to allow for more efficient calculations of a large number of states.

The Davidson-Olsen algorithm is the quasi-Newton method applied to the CI prob- 
lem. It uses an approximate Hamiltonian $H_{0}$, typically diagonal, to compute successive corrections to an approximate CI vector $\boldsymbol{C}_{j}^{(i)}$. The next CI vector $\boldsymbol{C}_{j}^{(i+1)}$ is then found by diagonalizing a small Hamiltonian in the basis of all CI vectors at the current iteration and their corresponding correction vectors. To improve convergence, the correction vectors of a few previous iterations are often included. When calculating a very large number of states, this scheme (hereafter called Version 1) encounters some problems. First, storing several hundred CI or correction vectors from a few iterations can put severe constraints on memory. Second, the higher-lying states typically get increasingly difficult to converge, which leads to a large number of CI iterations and makes the CI calculation very costly. Finally, as the number of previous correction vectors stored increases, they tend to have significant linear dependence, which can lead to numerical instability during the orthonormalization and sometimes even divergence.

The OpenMolcas CI algorithm has been significantly improved for calculations with hundreds of states. The first aim was to improve stability of the algorithm to better deal with linear dependencies in the correction vectors. A simple and quick fix in order to have a more reliable (but slower) convergence was to reduce the number of past iterations for which the CI vectors were saved to two instead of five. This version of the algorithm is called hereafter Version 2. As a more general alternative to this, a more comprehensive change of the code was realized. While the original implementation (Version 1) saved the last five correction vectors for each state, this new implementation instead sets an upper limit to the total number of correction vectors to around 400, which was found to be the best compromise between speed, flexibility and stability. Improvements were also made to reduce the risk of divergence in case of near-linear dependency by performing additional orthogonalizations and normalizations. This leads to what we call Version 3 of the algorithm. Finally, a very important improvement is to stop computing correction vectors for converged states. As lower roots converge significantly faster than the higher ones, this leads to a drastic reduction of the number of vectors to compute during the majority of the Davidson-Olsen iterations. This final version is referred to as Version 4 and is now the new default option in OpenMolcas. 
Savings in computational time with the modified CI algorithm depend on the size of the system and the active space. The improvement is most significant with a larger active space because then the CI step dominates the computational cost. As an example, we simulated the X-ray $\mathrm{K} \alpha$ emission spectrum of $\left[\mathrm{Fe}(\mathrm{bmip})_{2}\right]^{2+}$, a calculation with ten valence and four Fe 2p and 1s core orbitals in the active space (145 404 CSFs for the ground state), using up to 90 core-hole excited states in each irreducible representation, which was needed to get the desired orbitals in the active space. The calculation consists of 1 calculation of the initial 1 s core-ionized state and 3 independent calculations of the $2 \mathrm{p} \rightarrow$ 1s final states of different irreducible representations. RASSCF failed to converge in the old MOLCAS/OpenMolcas implementation (Version 1) because of numerical instabilities. The new algorithm does not suffer from such issues, and compared to the Version 2 calculation, the number of CI iterations is reduced by a factor of 3.6, while the saving in computational time is a factor of 4 , see fig. 13.269 Skipping the calculation of correction vectors for the converged low-lying states not only reduces significantly the computational time but also the number of iterations. The reason is that as the lowest states converge, more and more of the vectors used as basis for the Davidson diagonalization correspond to the remaining higher states, which can thus converge faster.

\subsection{Photoelectron spectroscopy and Dyson orbitals}

For the simulation of molecular ionization processes and photoelectron spectroscopy, it is routinely necessary to evaluate integrals between wave functions with $N$ and $N-1$ bound electrons. The information about the molecular system may then be conveniently compressed into a one-electron quantity

$$
\phi_{f i}^{\mathrm{DO}}\left(x_{1}\right)=\sqrt{N} \int \Psi_{f}^{N-1}\left(x_{2}, x_{3}, \ldots, x_{N}\right) \Psi_{i}^{N}\left(x_{1}, x_{2}, \ldots, x_{N}\right) d x_{2} \cdots d x_{N}
$$




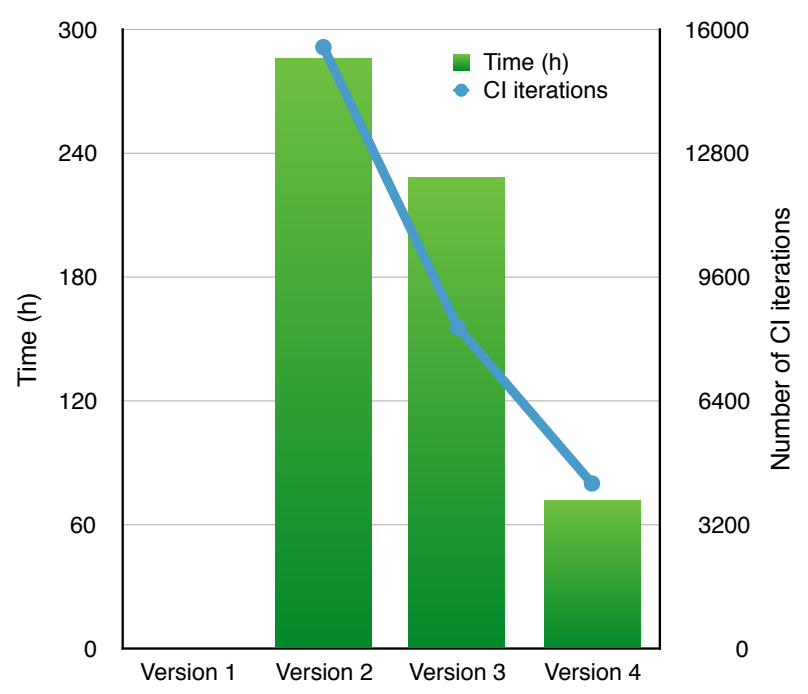

Figure 13: Total RASSCF time and total number of CI iterations for the calculation of X-ray emission spectrum of $\left[\mathrm{Fe}(\mathrm{bmip})_{2}\right]^{2+}$. Version 1 refers to the original algorithm. In Version 2, the number of past iterations for which the correction vectors are saved is reduced. Version 3 contains stability improvements and a cap on the number of stored vectors instead. Additionally, in Version 4, the corrections are not computed for the already converged lower-energy states.

commonly known as the Dyson orbital (DO). In the second quantization formalism, the Dyson orbital $\phi_{f i}^{\mathrm{DO}}$ may in a given orbital basis $\phi_{m}$ be computed as

$$
\begin{gathered}
\phi_{f i}^{\mathrm{DO}}=\sum_{m} c_{f i, m} \phi_{m} \\
c_{f i, m}=\left\langle\Psi_{f}^{N-1} \mid \hat{a}_{m} \Psi_{i}^{N}\right\rangle,
\end{gathered}
$$

where $\hat{a}_{m}$ annihilates a single electron from orbital $\phi_{m}$.

A new feature now natively available in the RASSI module is the calculation of multiconfigurational Dyson orbitals including spin-orbit (SO) coupling, similarly to what has previously been implemented with an external software. ${ }^{272[273}$ Within the RASSI framework for spin-free (SF) states, eq. (19) is efficiently evaluated within a biorthonormal $\operatorname{basis}^{274} \phi_{A}$ to obtain $c_{f i, A}^{\mathrm{SF}}$. As the basis $\phi_{A}$ may vary for different combinations of $f$ and $i$, the Dyson orbitals are subsequently re-expressed as $c_{f i, Z}^{\mathrm{SF}}$ in the atomic basis $\phi_{Z}$ through standard transformations. Dyson orbitals for spin-orbit coupled states are obtained as 
superpositions of those computed for the spin-free states

$$
c_{f i, Z}^{\mathrm{SO}}=\sum_{k, l} \xi_{f, k}^{*} \xi_{i, l} c_{k l, Z}^{\mathrm{SF}}
$$

where $\xi_{f, l}$ denotes expansion coefficient $l$ of spin-orbit state $f$ in the basis of the spinfree states. $\frac{163}{163}$ Both types of Dyson orbitals are, optionally, exported in Molden format for further use. The current implementation supports states obtained from RASSCF and RASPT2 calculations, both with and without the explicit use of symmetry.

Applied to photoelectron spectroscopy, the ionization cross section $\sigma_{f i}$ of the transition $\Psi_{i}^{N} \rightarrow \Psi_{f}^{N-1}$ is within the dipole approximation proportional to the squared photoelectron matrix element $\left|D_{f i}\right|^{2}$. Assuming a simple factorization $\psi_{\boldsymbol{k}}^{\mathrm{el}} \Psi_{f}^{N-1}$ for the final state and the strong orthogonality condition, 275 it can be written as

$$
\sigma_{f i} \propto\left|D_{f i}\right|^{2}=\left\langle\psi_{\boldsymbol{k}}^{\mathrm{el}} \Psi_{f}^{N-1}|\hat{\mu}| \Psi_{i}^{N}\right\rangle \stackrel{\text { str. orth. }}{=}\left|\left\langle\psi_{\boldsymbol{k}}^{\mathrm{el}}|\hat{\mu}| \phi_{f i}^{D O}\right\rangle\right|^{2},
$$

where $\psi_{\boldsymbol{k}}^{\mathrm{el}}$ is the continuum wave function of the ejected photoelectron. As $\psi_{\boldsymbol{k}}^{\mathrm{el}}$ is currently not representable within OpenMolcas, eq. (21) cannot be directly evaluated. However, assuming the transition dipole integral between $\psi_{\boldsymbol{k}}^{\mathrm{el}}$ and the normalized Dyson orbital to be constant, commonly called the sudden approximation (SA), the ionization cross section $\sigma_{f i}$ can be approximated by the squared norm of the Dyson orbital:

$$
\sigma_{f i} \propto\left|D_{f i}\right|^{2}=\left\|\phi_{f i}^{\mathrm{DO}}\right\|^{2} \underbrace{\left\langle\left.\left\langle\psi_{\boldsymbol{k}}^{\mathrm{el}}|\hat{\mu}| \frac{\phi_{f i}^{\mathrm{DO}}}{\left\|\phi_{f i}^{\mathrm{DO}}\right\|}\right\rangle\right|^{2}\right.}_{\approx \text { constant }} \stackrel{S A}{\approx A}\left\|\phi_{f i}^{\mathrm{DO}}\right\|^{2} .
$$

The SA cross sections obtained from eq. 22 are in the RASSI output referred to as Dyson amplitudes and provide a direct estimate of the relative cross sections. Note that the SA is inadvisable in cases where, e.g., the photoelectron has a low kinetic energy or the Dyson orbitals of the relevant transitions have strongly differing characters. Still, fig. 14 (a)-(c) illustrates how accurate photoelectron spectra may be obtained even for complex molecules from the RASSI Dyson amplitudes. Figure 14 (d) shows in addition 
how the shape of the Dyson orbitals facilitates spectral assignments. Recent publications have further demonstrated the applicability of the Dyson orbitals for simulation and analysis of photoelectron spectra $a^{272[273 \mid 276}-278$ and we expected them to find their use also for simulations of, for instance, Auger-electron and nonresonant X-ray emission spectra.

(a)

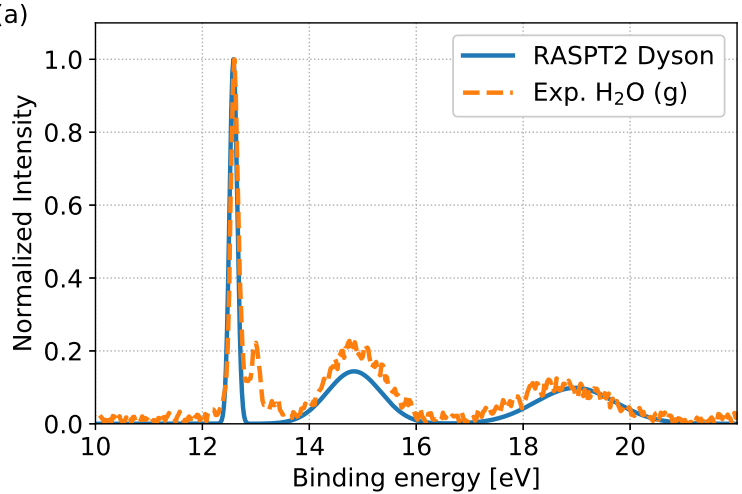

(c)

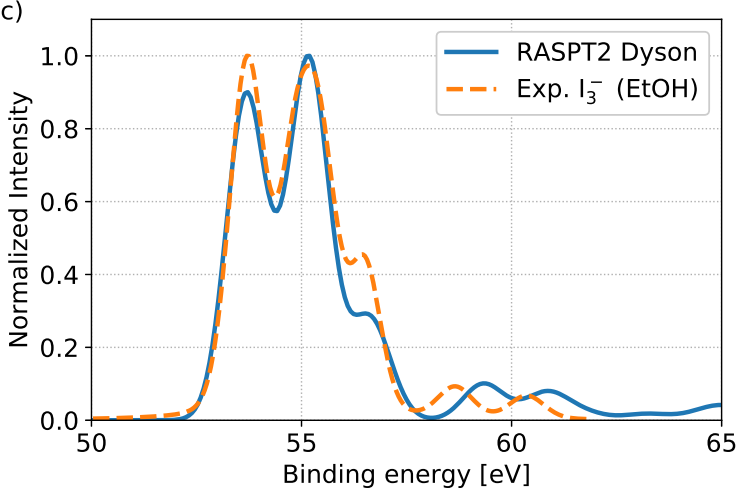

(b)

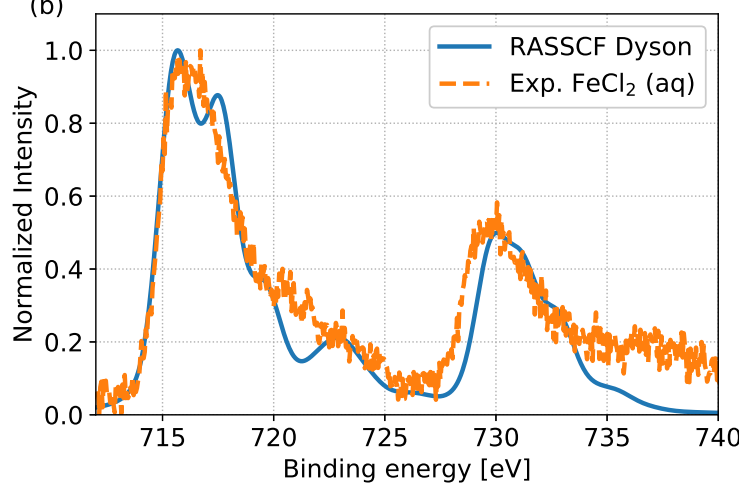

(d)

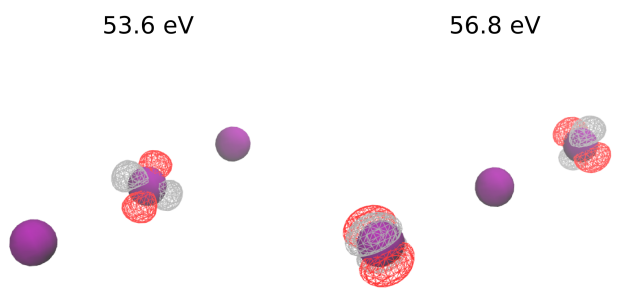

Figure 14: Comparison of photoelectron spectra obtained from Dyson amplitudes as implemented in RASSI and from cited measurements. (a) Valence extreme ultraviolet photoelectron spectrum of $\mathrm{H}_{2} \mathrm{O}(\mathrm{g}) \cdot \frac{272}{}$ (b) Fe2p X-ray photoelectron spectrum of $\left[\mathrm{Fe}\left(\mathrm{H}_{2} \mathrm{O}\right)_{6}\right]^{2+.272}$ (c) I4d X-ray photoelectron spectrum of $\mathrm{I}_{3}^{-.279}$ (d) Dyson orbitals (real part) for selected transitions $(53.6 \mathrm{eV}$ and $56.8 \mathrm{eV})$ from the $\mathrm{I}_{3}^{-}$calculation shown in panel (c), clearly assigning the features to I4d ionization from the central $(53.6 \mathrm{eV})$ and terminal $(56.8 \mathrm{eV})$ sites.

\subsection{Magnetic circular dichroism spectroscopy}

Magnetic circular dichroism ${ }^{280}$ (MCD) spectroscopy probes the differential absorption coefficient $\Delta \varepsilon$ for left versus right circular polarized light in the presence of a static magnetic field pointing in the light wave's propagation direction. It may reveal more information than regular absorption spectra, especially when spectral bands overlap, 281 because the MCD intensities of different transitions may have different signs. It is also able 
to provide information about the magnetic properties of the electronic states. However, MCD spectra can be complicated to analyze, and the analysis benefits greatly from theoretical support. With commonly applied approximations, the MCD for an electronic transition is given by

$$
\frac{\Delta \varepsilon}{E}=\gamma B\left[-\mathcal{A}\left(\frac{\partial f(E)}{\partial E}\right)+\left(\mathcal{B}+\frac{1}{k_{\mathrm{B}} T} \mathcal{C}\right) f(E)\right]
$$

Here, $\gamma=2 \mu_{\mathrm{B}} N_{\mathrm{A}} \pi^{3} \log e /(250 h c)$ in Gaussian units, and $f(E)$ is a line shape function in terms of the photon energy $E$. Equation $(23)$ and the expressions for the $\mathcal{A}, \mathcal{B}$, and $\mathcal{C}$ terms were first derived by Buckingham and Stephens. ${ }^{282 \mid 283}$ We focus here on the $\mathcal{C}$ term, which dominates the MCD for a molecule with a degenerate ground state, especially at low temperature. For a transition from a populated state $A$ with components $|A a\rangle$ and degeneracy $g_{A}$ to state $J$ with components $|J \lambda\rangle$, the isotropic $\mathcal{C}$ term in eq. 23 reads 280

$$
\mathcal{C}=-\frac{\mathrm{i}}{3 g_{A}} \sum_{a, a^{\prime}, \lambda}\left\langle A a^{\prime}|\hat{\boldsymbol{L}}+2 \hat{\boldsymbol{S}}| A a\right\rangle \cdot\left[\langle A a|\hat{\boldsymbol{D}}| J \lambda\rangle \times\left\langle J \lambda|\hat{\boldsymbol{D}}| A a^{\prime}\right\rangle\right] .
$$

Here, $\hat{\boldsymbol{L}}$ and $\hat{\boldsymbol{S}}$ are the dimensionless orbital and spin angular momentum one-electron operators, and $\hat{\boldsymbol{D}}$ is the one-electron electric dipole moment operator. The "nonrelativistic with spin" level of theory used for the operators in eq. (24) is commonly applied in calculations of absorption spectra and magnetic susceptibilities of compounds with even the heaviest elements, and therefore it should be adequate for MCD as well. An orbital angular momentum in the ground state is required for a nonvanishing $\mathrm{MCD} \mathcal{C}$-term spectrum. Consequently, if the ground state at the nonrelativistic or scalar relativistic (SR) level has only spin degeneracy, the $\mathcal{C}$ term is zero. In such a case, $\mathrm{MCD} \mathcal{C}$ terms may appear via spin-orbit coupling (SOC), if it mixes components of other - orbitally degenerate - spin states into the ground state. Other systems may already have a ground state orbital angular momentum in the nonrelativistic limit.

The functionality of OpenMolcas is very powerful for investigating MCD spectra theoretically. ${ }^{285}$ Ref. 284 reported the first ab initio wave function-based MCD calculation for an actinide complex, namely for the octahedral $5 \mathrm{f}^{1}$ system $\left[\mathrm{UCl}_{6}\right]^{-}$, using RASSCF 


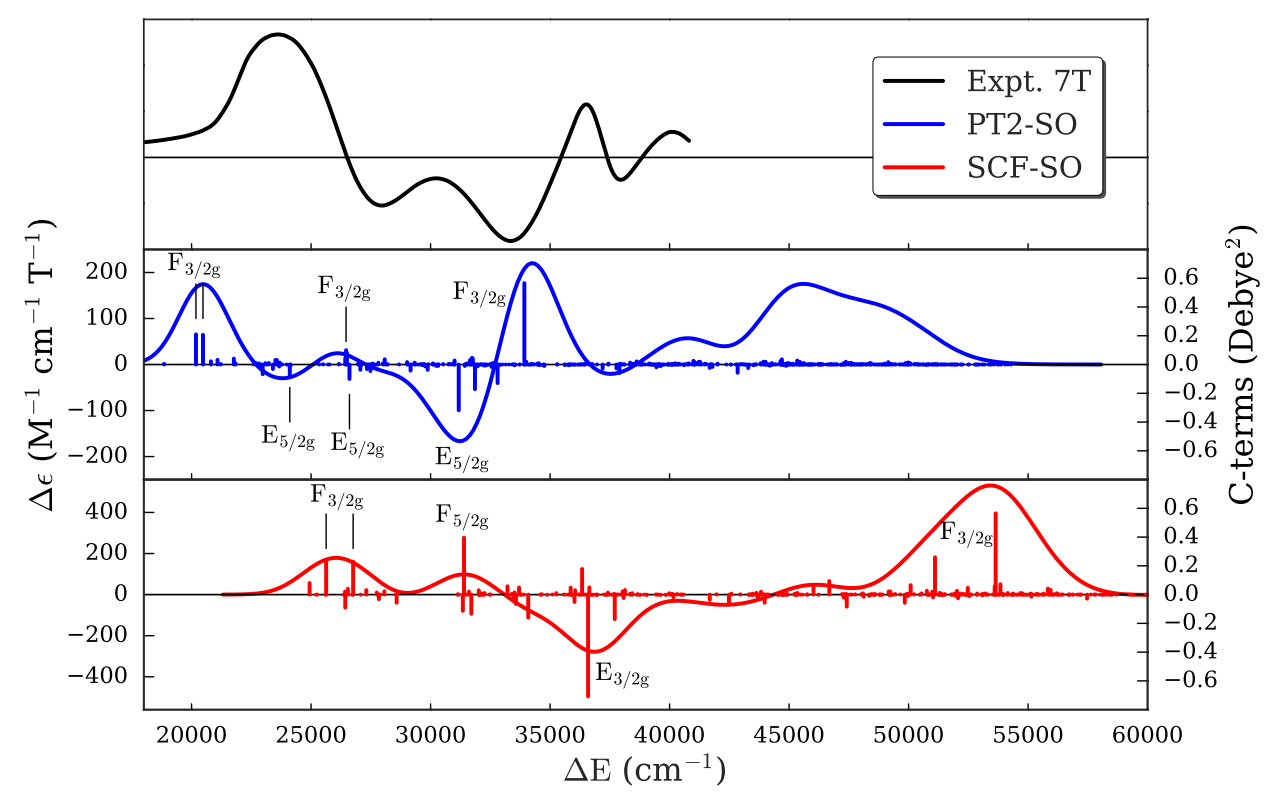

Figure 15: MCD spectrum $(5 \mathrm{~K})$ of $\left[\mathrm{UCl}_{6}\right]^{-} . \mathrm{MCD} \mathcal{C}$ term calculations based on RASSCF and RASPT2 calculations of doublet and quartet spin states and treatment of SOC by state interaction with the RASSI module. Calculated $\Delta \varepsilon$ generated from Gaussianbroadened vertical $\mathcal{C}$ term spectra. Experiment taken at $7 \mathrm{~T}$ field strength. Figure taken from ref. 284, Reproduced by permission of the PCCP Owner Societies.

and multi-state RASPT2 calculations followed by treating the SOC via state interaction. $\left[\mathrm{UCl}_{6}\right]^{-}$is one of the aforementioned cases where the $\mathcal{C}$ term spectrum is entirely a relativistic effect, i.e. due to SOC. The calculated and experimental UV-Vis range MCD spectra are compared in fig. 15. The PT2 spectrum, although over-correcting the blueshifted SCF spectrum, is in reasonably good agreement with the experimental MCD (also first reported in ref. 284). A collection of open-source utility codes and scripts ${ }^{286}$ was developed for the purpose of generating MCD intensities conveniently for a large number of transitions from SO-RASSI calculations. When the keyword PRPR is present in the RASSI input, the matrix elements for the electric dipole moment, the electron spin, and the orbital angular momentum for the calculated set of SO states are printed to a set of files, which is convenient for post-processing. The MCD intensities for vertical transitions, and broadened spectra, are then generated with the software tools of ref. 286. Code for $\mathcal{A}$ and $\mathcal{B}$ terms ${ }^{287}$ is also available in the repository. 


\subsection{Molecular magnetic properties}

In the field of molecular magnetism, the SINGLE_ANISO module ${ }^{8}$ has allowed the computation of the parameters of all magnetic Hamiltonians and the field- and temperaturedependent thermodynamic properties for mononuclear complexes and fragments. The POLY_ANISO module now allows the computation of the magnetic properties of polynuclear complexes using a semi-ab initio approach. The necessity for such an approach is dictated by the current computational difficulties with a full $a b$ initio treatment of polynuclear compounds containing several transition metals or lanthanide ions. In the proposed approach, the first step consists of dividing the investigated polynuclear compound in several mononuclear fragments containing one magnetic centre only. This fragmentation does not imply that the ligand framework needs to be altered: replacing a neighbouring magnetic centre with its diamagnetic closely-related metal ion suffices. For example, the effect of a neighbouring $\mathrm{Er}^{3+}$ site could be reasonably well simulated by a diamagnetic $\mathrm{Lu}^{3+}$, containing a completely filled $4 \mathrm{f}$ shell. Ab initio calculations using the SINGLE_ANISO module are then performed for each of such fragments. The features of POLY_ANISO can be divided in three groups: modeling of magnetic exchange interactions, decomposition of magnetic exchange interactions, and computation of magnetic thermodynamic properties. Both SINGLE_ANISO and POLY_ANISO also have the ability to generate in an automatic way various plots, via an interface to gnuplot utility. $\frac{288}{28}$

Modeling of exchange: The total magnetic interaction between two metal centers is a sum of the anisotropic exchange interaction and dipole-dipole magnetic interaction. The dipole-dipole magnetic interaction is evaluated exactly by using the total magnetic dipole moment matrix elements calculated on the basis of local multiplets of the corresponding metal sites available from the fragment SINGLE_ANISO calculations.

The easiest simulation of anisotropic exchange interaction is achieved within the Lines model, $\stackrel{289}{ }$ involving one effective exchange (Lines) parameter per interacting pair. This parameter quantifies the hypothetical isotropic exchange interaction between the groundstate spins of the corresponding magnetic ions, which would arise in the absence of spin-- 
orbit coupling. The anisotropic exchange is modeled via the projection of this effective isotropic model over chosen spin-orbit multiplets of individual metal centers obtained in the corresponding fragment SINGLE_ANISO calculations. The Lines model is expected to be accurate in three cases: (i) the two interacting sites have uniaxial (Ising) magnetic anisotropy (reduces to generally noncollinear Ising exchange), (ii) the two interacting sites are completely isotropic (obviously reduces to Heisenberg exchange); and (iii) one site is isotropic while the other possesses Ising magnetic anisotropy (reduces most often to collinear Ising exchange). $\frac{290}{2}$ For all other cases of intermediate anisotropy of interacting sites, the Lines model is believed to be a reasonable approximation. The Lines exchange parameters are the only unknown parameters in this approach and are usually found from a least-squares fit of the experimental magnetic data. Alternatively, the Lines exchange parameters can be extracted/estimated from broken-symmetry density functional theory calculations (BS-DFT). ${ }^{291292}$ To this end, the anisotropic magnetic ions are replaced by the closest isotropic metal ions. Then the extracted BS-DFT exchange parameters $J_{12}$ for a given pair of ions $(1,2)$ in such hypothetical complex is converted into the corresponding Lines parameter $J_{12}^{\mathrm{L}}$ for the original complex with the formula $J_{12}^{\mathrm{L}}=\left(S_{i 1} / S_{a 1}\right)\left(S_{i 2} / S_{a 2}\right) J_{12}$, where the terms $S_{i}$ and $S_{a}$ stand for the spin of the corresponding isotropic ion and the genealogic spin of the anisotropic metal ion in the original compound, respectively. This approach was validated on several compounds. 293

The POLY_ANISO module contains also several extensions going beyond the simple Lines model: (i) the three-axes exchange Hamiltonian containing three exchange parameters parameters for each interacting pair, corresponding to the diagonal form (i.e., written in the main exchange axes) of symmetric anisotropic exchange Hamiltonian; 294 (ii) antisymmetric or Dzyaloshinsky-Morya (DM) exchange interaction, requesting three parameters (the DM vector); (iii) the general anisotropic nine-parameter exchange model including both previous interactions; and (iv) biquadratic terms in the exchange interaction. ${ }^{294}$ Another extension of the Lines model concerns more involved (many-parameter) effective exchange interactions in the Lines approach instead of a single-parameter one.

The total magnetic interaction matrix is then written straightforwardly for the basis 
of the products of the on-site eigenstates obtained in the fragment ab initio calculations, and then diagonalized. $\sqrt[295]{ }$ The obtained eigenstates alongside with the spin and magnetic dipole moment integrals expanded in the obtained exchange-coupled basis, are then further employed for the computation of the parameters of all magnetic Hamiltonians and magnetic properties.

\section{Full decomposition of magnetic exchange interactions using irreducible ten-}

sor technique: The implemented models of magnetic interaction (e.g. Lines model) describe the exchange interaction between local pseudospins. ${ }^{296 \mid 297}$ The Lines parameters are not directly transferable between different compounds (given that their local pseudospins might differ). In order to overcome this problem, the full decomposition of all the interaction models implemented in POLY_ANISO program has been developed and implemented using the irreducible tensor technique, $\stackrel{298}{20}$ for all interacting pairs. As result, a set of parameters $J\left(k_{1}, q_{1}, k_{2}, q_{2}\right)$, where $k_{i}, q_{i}$ represent the rank and projection of the local irreducible tensor operator $O_{k_{i}, q_{i}}$ on site $i$. The interaction Hamiltonian can be recovered exactly by summing up all contributions (eq. (25)). The parameters are

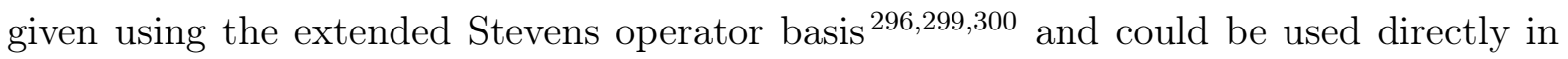
connection with the EasySpin function in MATLAB. 301

$$
\hat{H}_{\mathrm{exch}}^{1,2}=\sum_{k_{1}, q_{1}, k_{2}, q_{2}} J_{k_{1}, q_{1}, k_{2}, q_{2}} O_{k_{1}, q_{1}} O_{k_{2}, q_{2}} .
$$

Thermodynamic magnetic properties: The POLY_ANISO module is able to compute basic static magnetic properties for polynuclear compounds. For the computation of the molar magnetization, the Zeeman matrix is built on the basis of several low-lying energy states (user-defined size) and diagonalized. The resulting eigenstates are used to compute molar magnetization where Zeeman states are being populated according to the Boltzmann distribution law. Contribution to the magnetization arising from the states which did not enter explicitly into the Zeeman matrix is considered within the secondorder perturbation theory. The computation of molar magnetization (and torque) takes into account also the contribution of local excited states which were not accounted for 
exchange coupling. Molar magnetic susceptibility (tensor and powder) is computed using the zero-field limit of the molar magnetization, where the implemented expressions do not require the Zeeman Hamiltonian to be explicitly computed, enabling significant speedup in computation of these properties compared to the computation of molar magnetization. The magnetic susceptibility is computed in several formulations: (i) the derived formulas for zero-field limit, (ii) using the "experimental" formulation as $M / B$ (inaccurate but employed quite often), and (iii) as derivative of the magnetization with respect to applied field $\mathrm{d} M / \mathrm{d} B$ (i.e. the rigorous definition). Intermolecular interactions are accounted for in a mean-field approach, using a single parameter $(z J)$. This parameter is usually quite small in magnitude and influences magnetic susceptibility, torque and magnetization at very low temperatures. For each temperature point, the main values and main axes of the susceptibility tensor are given.

This methodology has been successfully applied for the investigation of anisotropic

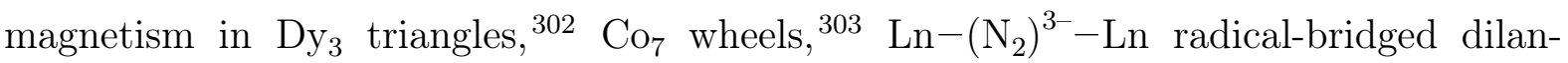
thanides $\sqrt{304}$ and many other compounds (see fig. 16). POLY_ANISO and SINGLE_ANISO are actively used also for the investigation of the structure of blocking barriers of single-molecular magnets, molecules which display magnetic bistability of intra-molecular origin.

\subsection{Ultra-high precision computer spectroscopy.}

The accuracy of computer simulations can challenge that of experiments, suggesting that it may be worth to compute rather than measure some properties. This alternative way to achieve accurate reference data is pursued by some scientists using the OpenMolcas platform. In this context we would like to mention the open-ended implementation of the OpenMolcas platform in handling one-particle electronic basis functions with high angular momentum, allowing the evalution of electron repulsion integrals and their storage in FCIDUMP format, using the same code used for the Stochastic-CASSCF approach, discussed in section 3.1. This feature has been used, for example, in the computational

prediction of the carbon atom's first ionization energy to within $1 \mathrm{~cm}^{-1}$ accuracy ${ }^{\sqrt[306]{ }}$ from 


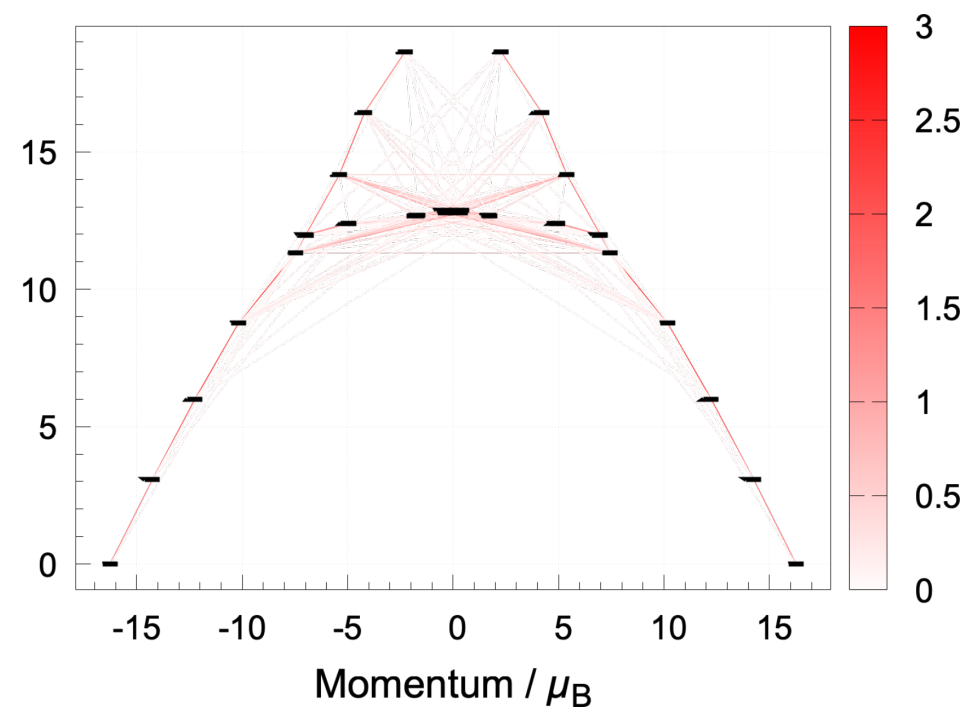

Figure 16: An example of application of the POLY_ANISO module for the $a b$ initio construction of magnetization blocking barrier of a $\mathrm{Co}$ (II)-Gd (III)-Co(II) trinuclear complex. ${ }^{305}$ The magnetic relaxation path, outlining the blocking barrier is traced by the red lines, whose intensity scale the transition magnetic dipole matrix elements between the connected multiplet states.

computer spectroscopy. $\stackrel{307}{[07}$ This calculation involved six fully correlated electrons in the neutral carbon atom, with an aug-cc-pCV8Z basis set (569 basis functions, including up to $l$ angular functions). In the language of CAS spaces, this would be a CAS $(6,569)$, which is by far the largest CAS calculation ever done to micro-hartree precision as far as we are aware.

\section{Analysis and visualization}

As discussed above, OpenMolcas offers a broad range of quantum-chemical methods to describe large molecules with many correlated electrons and orbitals, and to compute many excited electronic states efficiently. Setting up these calculations is a nontrivial task, requiring input from the user, who should have a sufficient understanding of the system under study and the method being applied. The analysis can be challenging if states with different characters mix, if multiconfigurational character and double excitations are present, or if a large number of states are to be analyzed.

To help the user prepare and analyze calculations, OpenMolcas contains, or is easily interfaced with, suitable tools. Apart from widespread capabilities, like the computation of 
Mulliken populations and electric multipole moments, OpenMolcas includes, for example, the LOPROP program $\frac{308}{3}$ for computing local properties and supports the generation of various types of orbitals - canonical, natural or localized with several methods. The generated orbitals, in the form of lists of coefficients, are usually not very informative, and some visualization tool becomes necessary in order to better appreciate the shape and structure of the orbitals. Traditionally this has been done by exporting the orbital and basis-set information in Molden ${ }^{309}$ format. However, it should be pointed out that there are several different ways to choose the orbitals (e.g. state-averaged and statespecific natural orbitals, and natural transition orbitals) and that it is not always clear which orbital representation is optimally suited. Therefore, a number of tools have been developed that allow to easily switch between these different representations.

Recent developments in OpenMolcas provide additional tools to visualize and manipulate the orbitals (section 6.1), as well as to analyze their properties (section 6.2), and more options for the analysis of wave functions and transitions, including tools that allow for a completely automatized analysis of the wave function character and provide a rigorous route to comparing the wave functions produced by different methods (sections 6.3 and 6.4). In this section we describe the most significant of these new capabilities.

\subsection{Graphical interface and orbital visualization}

OpenMolcas, as other high performance computing programs, requires numeric data as input and can produce a vast quantity of data as output. Raw numeric data (atomic coordinates, orbital coefficients, electron populations and bond orders, etc.) are often very difficult to comprehend. As a typical example, an essential step in CASSCF and related calculations is selecting the active space, a task for which an orbital viewer is of great help. With MOLCAS, the tools of choice have been either third-party programs capable of computing and rendering isosurfaces "on the fly", from the basis set and orbital coefficients information (in Molden format), or more specific programs which rely in the precalculation of the volumetric data and use custom formats.

First, in order to make these tasks easier, a program with GUI: LUSCUS has been de- 
veloped $^{310}$ as a stand-alone program (distributed under the Academic Free License (AFL) version $3.0^{(311}$ ) convenient for manipulation and visualization. Although some data produced by quantum chemistry programs can be stored in standardized or well-established formats, for complex data the formats are often unique and thus GUIs are strongly bound to the computational code. LUSCUS uses a native data format (see fig. 17) to read or write visualizable data, which is supported by OpenMolcas. Other chemical formats can be used with LUSCUS since they are automatically converted to and from LUSCUS data format by external plug-ins. Plug-ins convert data on demand without the need of user intervention. Therefore, from the user's point of view, LUSCUS is capable of manipulating a number of chemical formats, making it not only a graphical front-end for OpenMolcas, but also a powerful general-purpose chemical viewer and editor.

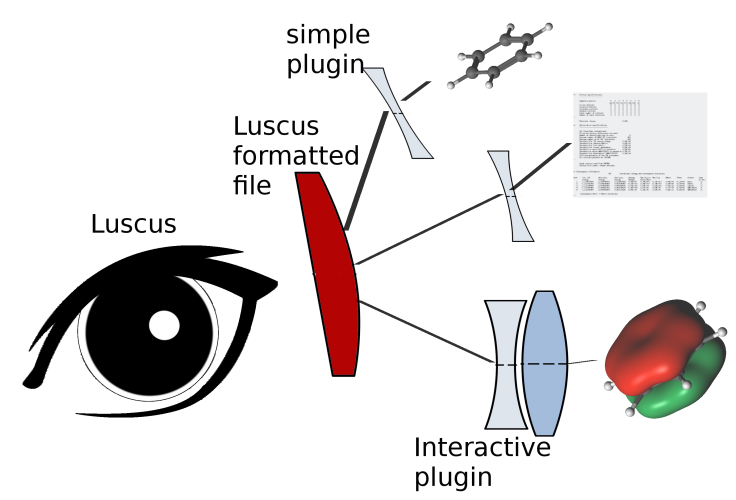

Figure 17: Interaction of LUSCUS and various data files: LUSCUS can only operate with .Ius files (the native LUSCUS format) and separate plug-ins (both simple and interactive) are used to convert the data.

Complementarily, a new stand-alone program has been developed to generate precomputed volumetric data: SAGIT (stand-alone grid independent transformer). ${ }^{312}$ This code is operated via command line and can be useful to generate volumetric data for many orbitals simultaneously, or in batch mode, where a GUI is not needed; it can also be used as a plug-in for LUSCUS to visualize orbitals on the fly.

With the release of OpenMolcas, a new orbital viewer has been developed which tries to suit the most typical needs of OpenMolcas users. Pegamoid $\frac{313}{3 s}$ is written in Python and is easily installable in modern computer systems. It can open orbital or volumetric files in 
a variety of formats generated by OpenMolcas. Most significantly, it can open, display and save orbital files in the new HDF5 format generated by OpenMolcas, which has several advantages compared to other formats: (i) as outlined in section 2 , it is a portable selfdocumented format that allows easy transfer and access of data, (ii) it is directly usable by OpenMolcas programs, e.g. as starting orbitals for the RASSCF program, (iii) it is not affected by limitations in the Molden format, like the maximum angular momentum in basis functions or the mixture of Cartesian and spherical harmonic type of functions. With respect to formats with volumetric data, the main advantage is that its size is not excessive even with large basis sets, and that the desired quantities to be visualized need not be precomputed.

Apart from simply viewing the orbitals saved in a particular file, Pegamoid (as well as LUSCUS) can also assign them to different orbital spaces (frozen, inactive, RAS1, RAS2, RAS3, secondary, deleted) and save them in a new file that can be used as input for OpenMolcas programs. When the orbital file contains the necessary information (e.g. an HDF5 file generated by a state-average RASSCF run), it is also possible to visualize, without further post-processing, natural orbitals and occupations for the different electronic states, electron density differences, natural difference orbitals and natural transition (or binatural) orbitals between the states ${ }^{314}$ (see fig. 18).

Pegamoid's goal is to be a quick and convenient tool for viewing orbitals, selecting active spaces and identifying states. While it has options to control the display quality and features, the generation of high-quality eye-catching pictures often requires the use of other packages that offer a wider range of options. For this purpose, Pegamoid can be used to save the desired volumetric data in the Gaussian . cube format, 21 which can then be used by other programs to render pictures with customized colors, textures, backgrounds and additional elements.

\subsection{Property integrals of individual orbitals}

OpenMolcas supports the computation of property integrals of molecular orbitals in the SEWARD module, including the multipole moments, Cartesian moments, electric po- 


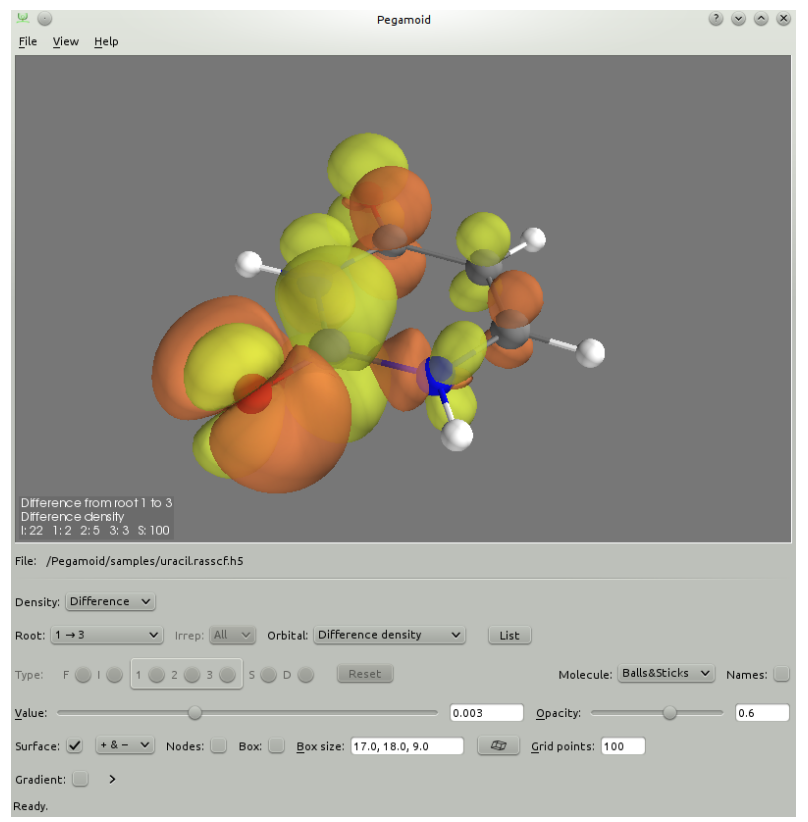

Figure 18: Pegamoid interface, showing the difference density between two electronic states of uracil (an $n \rightarrow \pi^{*}$ transition).

tential, electric field, electric field gradients, diamagnetic shielding, one-electron Darwin contact term, etc. It supports any orbitals calculated in SCF, RASSCF, CASPT2 or other modules. With natural orbitals, the sum of the occupation-number-weighted orbital property integral is the total electronic part of a property integral, so the occupation-numberweighted orbital property represents how much each orbital contributes to a property integral of an electronic state. For analysis purposes, one has the option of printing, for each molecular orbital, these property integrals weighted by the occupation number of each orbital, or printing these property integrals for each orbital without the weighting. In case one is insterested in the property integral for an unoccupied orbital, the nonweighted option is required, as otherwise any property integral would trivially be zero. This can be useful when analyzing Rydberg states to determine which orbitals are Rydberg orbitals. It can also be helpful when deciding which orbitals to include in the active space of an MCSCF calculation and when determining whether the excited states from different MCSCF calculations correspond to the same state. ${ }^{315}$ For these examples, to determine how diffuse each orbital is, the second Cartesian moment $\left\langle r^{2}\right\rangle$ and its components $\left(\left\langle x^{2}\right\rangle,\left\langle y^{2}\right\rangle\right.$, $\left.\left\langle z^{2}\right\rangle,\langle x y\rangle,\langle y z\rangle,\langle x z\rangle\right)$ of each orbital can be calculated. An example input file making use of this capability can be found in the Supporting Information. 


\subsection{Binatural or natural transition orbitals for excitation char- acterization}

There is a frequent need to quantify the difference between two states, and to visualize the difference for easily understood characterization. Assuming we have at hand correlated electronic wave functions, the usual difference density is often insufficient. However, recalling that the usual natural orbitals are the eigenfunctions of the one-electron reduced density matrix of a single state, a simple extension is to use the singular value decomposition (SVD) of the transition density matrix of two states to characterize their difference. This approach was used for single-determinant wave functions ${ }^{316}$ and for TDDFT and CIS wave functions, $\frac{317}{}$ where the orbitals were named natural transition orbitals, and independently by Mayer. $\frac{318}{}$ An SVD has also been used to relate orbitals of fragments of a composite molecule. ${ }^{319}$ Prior usage seems to have been proposed mostly for specific applications, but in ref. 320 it was suggested to use orbitals computed as singular vectors of the one-electron transition density matrix as a technical tool to analyse the difference of pairs of general states, regardless of whether these were single-determinant states or indeed if they were actually computed to be eigenstates of any Hamiltonian. For general discussion of the SVD itself, see e.g. the articles by Stewart. 321322

The binatural orbitals, or natural transition orbitals, are obtained as the left and right singular functions of the reduced one-electron transition density matrix, $\gamma^{P Q}$. The binatural orbitals and their associated amplitudes encode in a compact way the whole $\gamma^{P Q}$ and can be used to analyze the transition between two electronic states.

The states would usually be either two noninteracting, computed states, or they can be two different approximations. They could also be e.g. states computed with and without some perturbation, or indeed any two states that allowed us to compute the transition density matrix in a suitable basis - maybe, but not necessarily obtained as an orbital basis that was used for the two states. The transition density matrices and the binatural orbitals are computed by the RASSI program within OpenMolcas.

There is one limitation: what is obtained is the difference of the states as expressed by a one-electron operator. One can easily extend this to form two-particle "binatural 
geminals". Also, one might have use for "Dyson amplitudes", as seen in section 5.3 , or "one-particle-two-holes" amplitudes, e.g. for core hole states.

\subsection{Detailed analysis of multireference wave functions}

The wave function analysis module $\mathrm{WFA}^{323}$ does not only provide visualization methods but also computes a number of quantitative descriptors with the aim of eliminating personal bias in the assignment of state characters and allowing for an automated analysis of large data sets. WFA is based on the open-source wave function analysis package libwfa. $\frac{314324}{324}$ One focus of libwfa is the analysis of excited states in terms of two-body electron-hole distributions within exciton theory. $\frac{325}{3}$ To visualize the excitations in real space, binatural or natural transition orbitals $\frac{317326}{32}$ and the more compact electron and hole densities $\frac{314}{31}$ are available. Electron-hole correlation effects can be elucidated using population analysis techniques. $\frac{326 \mid 327}{}$ Central tools of libwfa are excited-state descriptors quantifying spatial and statistical properties of excited states such as exciton size, $\underline{325}$ charge-transfer distance and correlation coefficient. $\frac{324}{}$ Furthermore, it supports the analysis of one-electron difference density matrices (1DDM) between different states, which is particularly important to study orbital relaxation effects. $\frac{32313281329}{}$ Finally, an effective number of unpaired electrons $s^{330 \mid 331}$ can be computed from natural orbital occupations. The WFA module is interfaced to the SCF, RASSCF, and RASSI modules of OpenMolcas. Through the RASSI interface, also other modules can be accessed such as the CASPT2 module and QCMAQUIS (DMRG and DMRG-NEVPT2, see section 3.2.).

Previously, the described analysis methods were used to analyze single-reference computations addressing a wide range of phenomena, such as the analysis of electronic delocalization in DNA, ${ }^{332}$ excitonic band structure in conjugated polymers, $\frac{327}{3}$ and the visualization of solvent effects in push-pull systems. ${ }^{333}$ We hope that through the new interface between OpenMolcas and libwfa, enabling the use of multireference methods, this scope can be significantly extended. Furthermore, the implemented analysis methods provide a rigorous route toward benchmarking excited-state calculations ${ }^{334 / 335}$ and can give new insight into specific failures of the different computational protocols employed. $\frac{329 \mid 336}{3}$ 

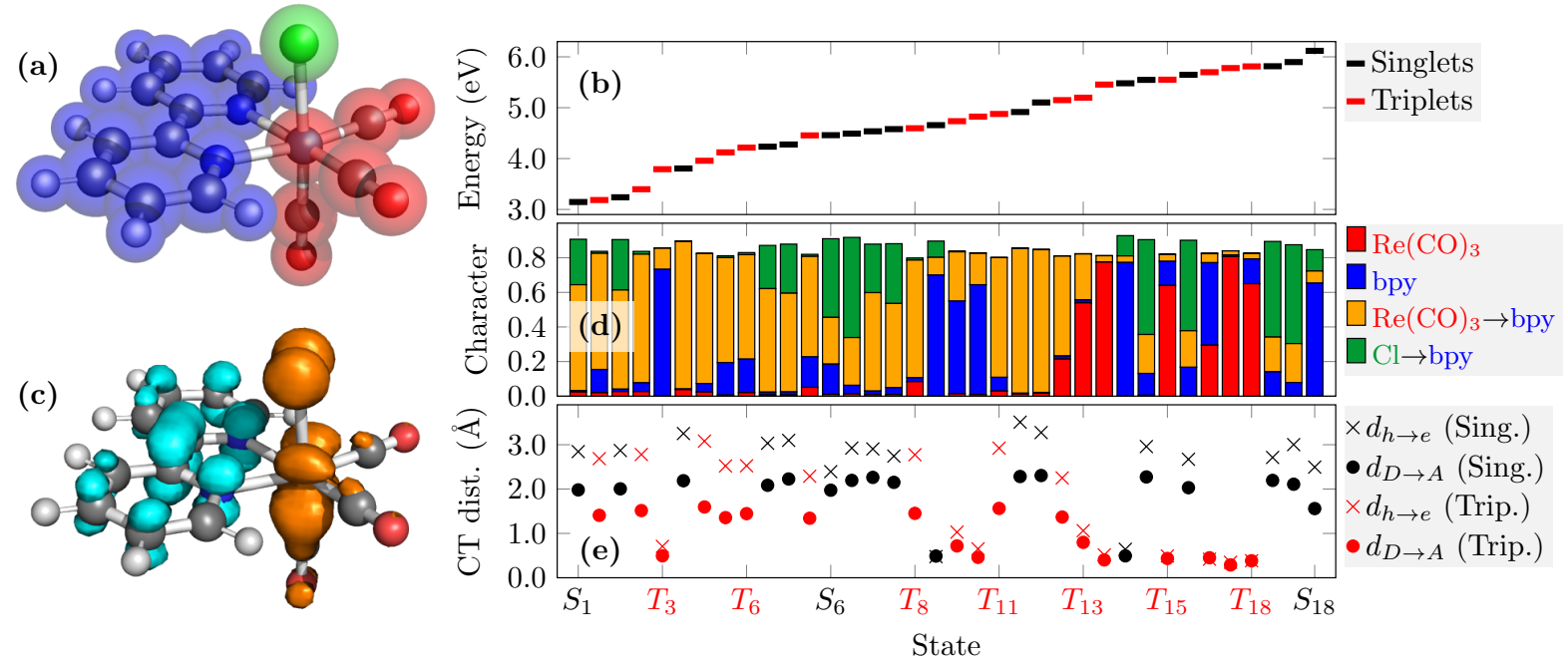

Figure 19: Analysis of the singlet and triplet excited-states of the $\operatorname{Re}(\mathrm{CO})_{3}(\mathrm{bpy}) \mathrm{Cl}$ complex computed at the MS-CASPT2 level using the WFA module: (a) molecular structure and definition of the $\mathrm{Re}(\mathrm{CO})_{3}$ (red), bpy (blue), and $\mathrm{Cl}$ (green) fragments; (b) excitation energies; (c) electron (cyan) and hole (orange) densities of the $S_{1}$ state; (d) decomposition into different local and charge transfer contributions; (e) charge transfer distance using two different descriptors.

Presenting a practical application of the WFA module, the excited states of the transition metal complex $\mathrm{Re}(\mathrm{CO})_{3}$ (bpy) $\mathrm{Cl}$ are analyzed (fig. 19 ). Computations were performed at the MS-CASPT2 level using a CAS(12,12) active space considering 19 singlet and 18 triplet states. The excitation energies of these states are presented in fig. 19 (b), illustrating the high density of low-lying states in this system. A direct analysis of these states in terms of canonical orbitals is not only tedious but is also quite challenging as the effects of different interacting configurations have to be disentangled. Therefore, we present here three alternative routes of analysing these states, as implemented in the WFA module: a visual analysis of electron and hole densities, a fragment-based decomposition of the excitations, and an analysis in terms of statistical descriptors.

As a first option, the densities of the excited electron and the excitation hole, derived from the one-electron transition density matrix (1TDM), $\frac{314}{2}$ are computed. These densities, computed for the $S_{1}$ state of the complex, are shown in fig. 19 (c). This representation shows the main state character in an intuitive way, i.e. the hole (orange) is located on the $\mathrm{Re}$ and $\mathrm{Cl}$ atoms whereas the electron (cyan) is located on the bipyridyl (bpy) ligand. However, the problem of any visual analysis is the required time and the 
dependence of the outcome on the subjective reasoning of the person analysing.

To overcome these problems of visual analysis, a completely automatized way to assign the state-character in transition metal complexes has been developed ${ }^{329 \mid 335}$ and it is presented as a second option here. First, the system needs to be divided into different fragments; in the present case a useful partition is the $\operatorname{Re}(\mathrm{CO})_{3}$ unit (red), the chlorine atom (green) and the bpy ligand (blue), as represented in fig. 19 (a). Subsequently, the excitation process is partitioned into different local contributions on the individual fragments and charge transfer (CT) between them using a 1TDM population analysis procedure yielding the so-called charge-transfer numbers. $\frac{314326}{326}$ The outcome of this analysis is presented in fig. 19 (d). The left-most bar graph corresponding to the $S_{1}$ state shows that this state is predominantly of $\operatorname{Re}(\mathrm{CO})_{3} \rightarrow$ bpy CT character (yellow bar, $61 \%$ ) with a secondary contribution of $\mathrm{Cl} \rightarrow$ bpy character (green bar, $26 \%$ ). More generally, fig. 19 (d) shows the low-energy states to be generally of $\operatorname{Re}(\mathrm{CO})_{3} \rightarrow$ bpy character with the exception of $T_{3}$, which is a locally excited state on bpy (blue bar).

A downside of the $\mathrm{CT}$ number analysis is that its application requires an a priori definition of the fragments used for the decomposition. When intuition is untrustworthy, it is possible to automatize the fragment definition process. ${ }^{335}$ Alternatively, we can move away from the population-analysis concept altogether leading to the third option, exemplified here through the computation of a CT distance in real space. Two different formulae are employed considering either (i) the distance $d_{h \rightarrow e}$ between the barycenters of hole and electron densities as computed from the 1TDM or (ii) the distance $d_{D \rightarrow A}$ between the barycenters of the detachment and attachment densities ${ }^{337}$ as computed from the $1 \mathrm{DDM} .324$ The results are shown in fig. 19 (e) and, without going into too much detail, we want to mention two observations. First, the CT distance mirrors CT character as identified in fig. 19 (d), i.e. the CT distances of the local states (red and blue bars) are close to zero while they are significantly enhanced for the CT states. Second, CT distances obtained from the 1TDM and 1DDM differ significantly where the latter are always lower. This difference can be interpreted to originate from orbital relaxation

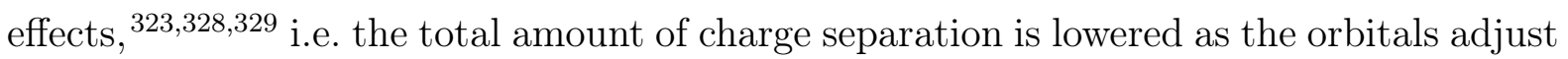


to the primary excitation process.

\section{$7 \quad$ Additional features}

In this final section on new options and utilities in OpenMolcas, we present developments for a new multiscale option, accurate and compact basis sets, symmetrization of wave functions, and simulations of muonic molecular systems.

\subsection{Multiscale simulations by frozen-density embedding theory}

The toolbox of electron correlation methods present in OpenMolcas would not be complete without the possibility to combine them in a multiscale fashion. Among the different possibilities for multiscale modeling in OpenMolcas, the one based on frozen-density embedding theory $(\mathrm{FDET})^{[38 \sqrt{340}}$ provides a robust tool to treat systems and problems of different nature. This multiscale approach is a first-principles description of a complex system as combination of two interacting subsystems. One portion of the system, subsystem $A$, is selected to be described at the molecular orbital level - e.g., through a Kohn-Sham determinant, $\frac{338}{2}$ or an interacting wave function $\sqrt[339]{ }$ - whereas the second (subsystem $B$, so-called "environment") is accessed through a simpler descriptor, namely its electron density. The interaction between the electrons of $A$ with subsystem $B$ is nonetheless accounted quantum mechanically through the action of the following (orbital-free) embedding potential:

$$
\begin{aligned}
v_{\mathrm{emb}}\left[\rho_{A}, \rho_{B}, v_{B}^{\mathrm{nuc}}\right](\boldsymbol{r})= & v_{B}^{\mathrm{nuc}}(\boldsymbol{r})+\int \frac{\rho_{B}\left(\boldsymbol{r}^{\prime}\right)}{\left|\boldsymbol{r}^{\prime}-\boldsymbol{r}\right|} \mathrm{d} \boldsymbol{r}^{\prime} \\
& +\frac{\delta T_{s}^{\mathrm{nad}}\left[\rho_{A}, \rho_{B}\right]}{\delta \rho_{A}(\boldsymbol{r})}+\frac{\delta E_{\mathrm{xc}}^{\mathrm{nad}}\left[\rho_{A}, \rho_{B}\right]}{\delta \rho_{A}(\boldsymbol{r})}+\frac{\delta \Delta F\left[\rho_{A}\right]}{\delta \rho_{A}(\boldsymbol{r})} .
\end{aligned}
$$

The first two terms in eq. (26) represent electrostatic contributions due to the nuclei of system $B$ and to its electron density $\rho_{B}(\boldsymbol{r})$, respectively. The next two terms are instead purely quantum mechanical and originate from the nonadditive contribution to the noninteracting kinetic energy functional $T_{s}[\rho]$ and the exchange-correlation functional 
$E_{\mathrm{xc}}[\rho]$, respectively. By nonadditive contribution of a generic density functional $G[\rho]$, we refer to a bifunctional of the electron densities of $A$ and $B$, namely to $G^{\text {nad }}\left[\rho_{A}, \rho_{B}\right]=$ $G\left[\rho_{A}+\rho_{B}\right]-G\left[\rho_{A}\right]-G\left[\rho_{B}\right]$. Finally, the last term in eq. (26) is not related to the interaction between the two subsystems, but it rather ensures consistency with the Hohenberg-Kohn theorem upon introduction of approximations in the description of subsystem $A$. This term is however neglected in most practical applications of the theory. $\underline{339}$

The one-electron operator corresponding to the potential of eq. (26) can then be added to the environment-free Hamiltonian $\left(\hat{H}_{A}\right)$ of the quantum mechanical method of choice for subsystem $A$ in order to proceed with the electronic structure calculation. Naturally, this way to proceed simplifies the computational effort compared to the corresponding quantum mechanical calculation on the entire $(A+B)$ system. One limitation of the current OpenMolcas implementation is the need to use explicitly basis functions on $B$ as well as $A$. For true multiscale simulations, where for example $\rho_{B}(\boldsymbol{r})$ is obtained from statistical averages, $\stackrel{341}{\text { the }}$ use of a real-space representation is certainly desirable, and will be implemented in a future release of the software. Nonetheless, it should be pointed out that the overhead for the presence of basis functions on $B$ is somewhat handled intelligently so that correlation methods need not to include orbitals from subsystem $B$ explicitly (see section S15).

If the exact functional forms for $T_{s}^{\mathrm{nad}}\left[\rho_{A}, \rho_{B}\right]$, for $E_{\mathrm{xc}}^{\mathrm{nad}}\left[\rho_{A}, \rho_{B}\right]$ and for $\Delta F\left[\rho_{A}\right]$ were known, eq. (26) would produce an exact quantum mechanical model for the interacting subsystems, but in practice various approximations to these functionals are available that guarantee a sufficient accuracy. Some of such approximate functionals are included in the current OpenMolcas implementation of FDET ${ }^{342}$ as detailed in the software documentation. Noticeably, the construction of the embedding potential through eq. 26 requires the user to make another important choice, namely that of which $\rho_{B}(\boldsymbol{r})$ is to be used. This is a key issue for the correct use of FDET, and despite the lack of a general answer to the question, the many studies present in the literature show the general trend that the results do not depend strongly on the choice of $\rho_{B}(\boldsymbol{r})$. $\frac{346}{}$ In practice, with any physically justified $\rho_{B}(\boldsymbol{r})$, deviations in the predicted oservables are smaller 
than the errors introduced by other sources, such as the basis set used, the choice of the approximants for $T_{s}^{\mathrm{nad}}\left[\rho_{A}, \rho_{B}\right]$ and $E_{\mathrm{xc}}^{\mathrm{nad}}\left[\rho_{A}, \rho_{B}\right]$, or the quantum mechanical method selected to describe subsystem $A$.

As OpenMolcas is specialized in multiconfigurational wave function methods, ${ }^{7}$ the combination with FDET represents a somewhat unique tool for investigating complex systems especially in their excited states and for notorious DFT-hard situations. With this in mind, an effort has been put into the development of a variant of FDET, known as linearized FDET, $\stackrel{343}{ }$ that shows some advantages compared to the conventional FDET approach, as it inherits useful properties of the corresponding wave function method. Linearization consists of approximating the nonadditve functionals so that they become linear in $\rho_{A}(\boldsymbol{r})$, by means of a Taylor expansion around a reference density $\rho_{A}^{\text {ref }}(\boldsymbol{r})$, which can be assumed not to differ significantly from the stationary density of the embedded subsystem. Among the many advantages of linearized FDET, we point out the fact that the eigenstates of the embedded Hamiltonian are orthogonal, hence they can be directly related to the true ground and excited states of the embedded subsystem. Also, differences in the computed eigenenergies of the embedded Hamiltonian can be used to assign excitation energies in much the same way as it is done for the calculation on the isolated system $A$.

\subsection{Development of new ANO basis sets}

Basis sets based on the atomic natural orbitals (ANO) approach $\stackrel{347}{ }$ are the workhorse of the MOLCAS/OpenMolcas program packages. The ANO contraction scheme offers a fast convergence towards the uncontracted form of the basis set. Thus, ANO basis sets even in small contracted forms - say valence double zeta plus polarization functions (VDZP) - usually are able to yield accurate results, which makes them the perfect choice for calculations where the bottleneck lies in the correlation part. With the focus on fast convergence by contraction and application in accurate correlated calculations, ANO basis sets were allowed to contain a larger number of primitive basis functions than other comparable basis sets. .3481349 
Accompanying the nonrelativistic ANO-L basis set, $\frac{350}{30}$ the more compact ANO-S basis set ${ }^{3511}$ had been available, thus allowing more economic treatment of large molecular systems where the integral computation time can rival the correlation part. For relativisitic calculations, however, so far only the relativistic core-correlated ANO-RCC basis set $\underline{352}$ had been developed for the second-order Douglas-Kroll-Hess (DKH2) Hamiltonian. The large size of this basis set in terms of primitive basis functions made integral calculations for extended systems rather costly. To make also relativistic calculations for large systems more economic, a new compact relativistic basis set, the ANO-XS basis set, $\underline{353}$ has recently been developed. The number of primitive basis functions in the ANO-XS basis set is about half that of the ANO-RCC basis set. This greatly reduces the computational costs in the integral computation time, especially when used in combination with Cholesky decomposition.

The ANO-XS basis set is available for light atoms $(Z \leq 20)$. Its main intent is to be used in combination with the larger ANO-RCC basis set in relativistic calculations containing heavy atoms such as the chromium complex shown in fig. 20, constituting a part of a metal-organic framework (MOF). When using the ANO-RCC basis set with VDZP contraction for all atoms, the total number of primitive basis functions is 4573 . Replacing the basis set for all atoms except chromium with the ANO-XS basis set reduces the number to 2014.

\subsection{Symmetrization of wave functions}

At the early stage of quantum chemistry, the symmetry of molecules played an important role as an efficient way to reduce the size of the computational problems. Today this aspect is not as important, since the absolute majority of computed systems have no symmetry elements except of identity.

Many computational codes, including OpenMolcas, use only Abelian point groups ( $D_{2 \mathrm{~h}}$ and subgroups) for calculations. In this case the result of any symmetry operation on a matrix element is a multiplication by 1 or -1 .

If a molecule has a symmetry higher than $D_{2 \mathrm{~h}}$, the computational code will use a 


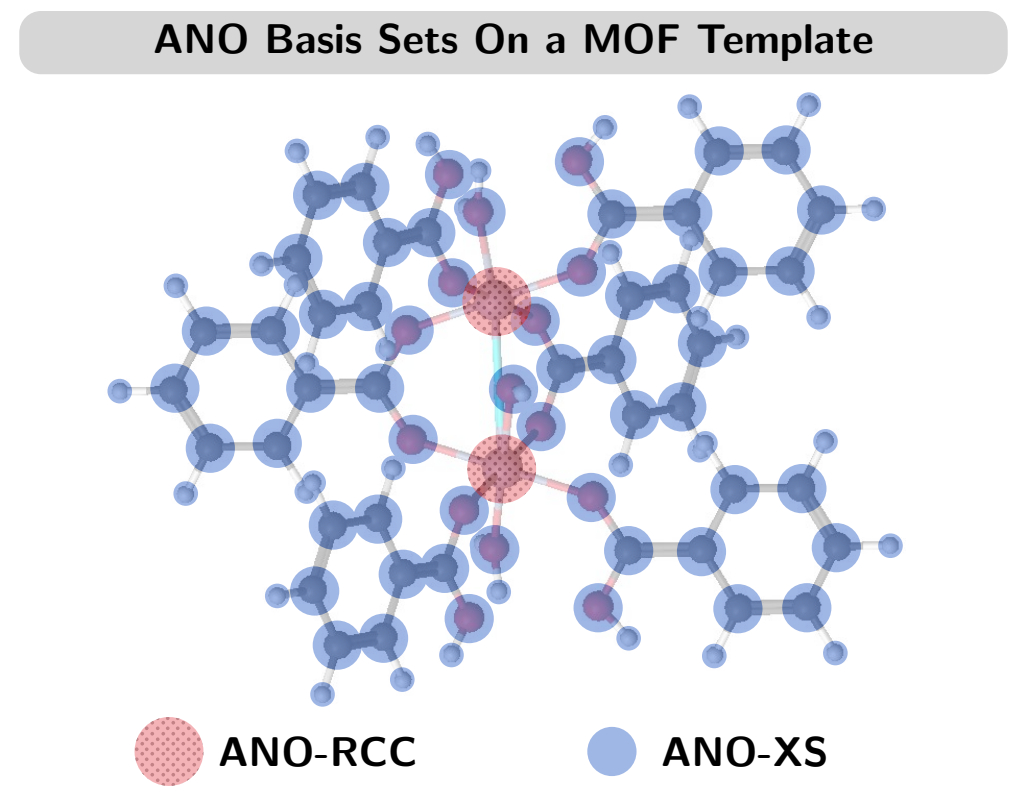

Figure 20: Basis set combination of ANO-RCC and ANO-XS for the calculation of a MOF template.

lower symmetry and as a result, the computed density or wave function can present a broken symmetry. In some cases, this deviation from symmetrical solution will be small, but in other cases this error will be significant,

Rewriting the computational codes for the purpose of handling an arbitrary point group is a rather complicated programming task. Instead, there is a more simple approach, which involves symmetrization of the computed wave function according to any specified symmetry, e.g. the symmetry of the molecular structure. The procedure of symmetrization of the wave function can be applied at the end of the calculation, or during the iterative procedure of computing the wave function (e.g. in a self consistent cycle). The details of the algorithm of symmetrization are described in ref. 354. Prior to an SCF calculation the point group is determined. The orbital spaces for each irreducible representation (irrep) are then calculated for the supplied basis. At each iteration the symmetric orbitals are projected out and partner functions determined. Coefficients are then averaged over the partner functions. Since the dimension of each irrep space is known, the symmetrization step will also verify that the projected subspaces belong to the correct symmetry species. This is required since no restriction is placed on the rotations during the iterations, and large symmetry breaking may occur. 
A mathematical library (libMSYM) has been designed for two independent tasks: automatic detection of the point group, and symmetrization of the computed wave function according to a given symmetry $\sqrt[355]{ }$ Version 2.0 of the library can be integrated into the OpenMolcas code, and produce SCF calculations with wave function symmetrized at any SCF cycle. The usage of libMSYM is especially important if approximate integrals are used in the calculations. Two examples below demonstrate the abilities of the library.

As a first example, in tetrafluoromethane $\left(\mathrm{CF}_{4}\right)$, belonging to the $T_{\mathrm{d}}$ point group, the HOMO orbitals belong to the $\mathrm{T}_{1}$ irrep. After removing three electrons from the molecule and running a UHF/DFT calculation the symmetry will break even though $\mathrm{T}_{1}$ is 3 dimensional. By applying symmetry restrictions in the SCF calculation the charges will be evenly distributed between 3 orbitals of $T_{1}$ symmetry as can be seen in fig. 21 .
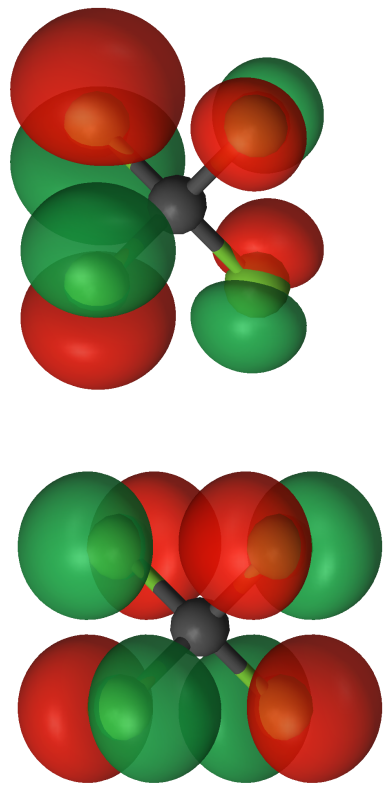
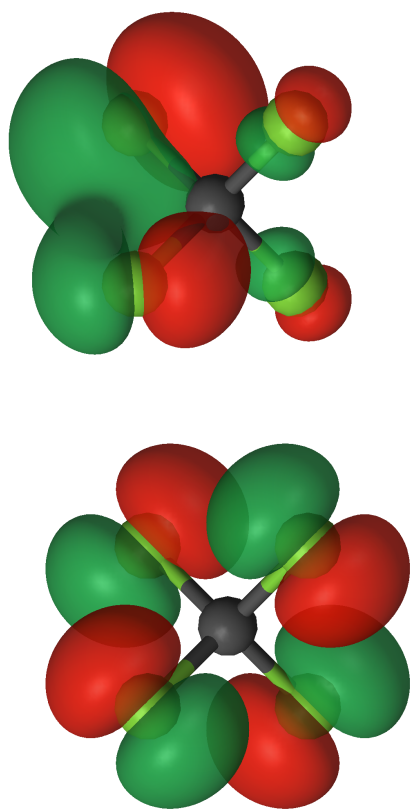
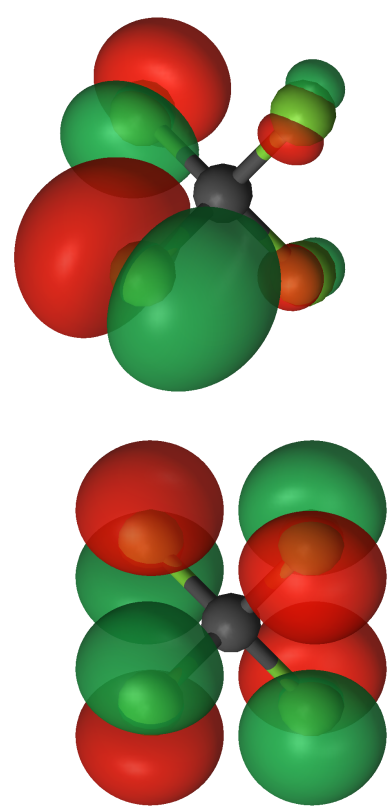

Figure 21: Orbitals for $\mathrm{CF}_{4}$ (point group $T_{\mathrm{d}}$ ) before (upper row) and after (lower row) symmetrization.

The second example is a symmetry-broken solution. The structure of the $\left(\mathrm{NO}_{2}\right)_{2}$ dimer is totally symmetric, and belongs to the $D_{2 \mathrm{~h}}$ point group. However, if we add a positive charge, the wave function (at UHF level) becomes unstable and the electron is localized at one of the monomers. By enforcing the symmetry with the libMSYM library, we obtain a fully symmetric solution. The total dipole moment is of the order of $10^{-13} \mathrm{D}$. 


\subsection{Calculations of muonic atoms and molecules}

The overwhelming majority of chemical studies, experimental and computational, are concened with "normal" matter, constituted of electrons, protons and neutrons. However, a large number of other elementary and composite particles are known which could, in principle, replace the ordinary fermions, although their lifetimes are usually so short that the practical significance is doubtful. An outstanding case is that of the muon, whose lifetime of $2.2 \mu \mathrm{s}$ has allowed its detection combined with normal matter. The muon is a lepton, with similar properties to the electron, but with a mass about 200 times larger. Muons can replace electrons in normal molecules and, due to their heavier mass, their density is concentrated much closer to the nuclei. The radiation emitted as a muon falls down to lower energy levels can give information about the nuclear charge distribution and, to some extent, about the electronic structure. Recent developments in muonic Xray spectroscopy for nondestructive elemental analysis $\sqrt[356]{358}$ have spurred the interest of theoreticians to predict the properties of muonic atoms and molecules.

Calculations of systems including electrons and/or muons are now possible with OpenMolcas. $\frac{359}{35}$ There are a few issues to take care of. One-particle and exchange integrals are always vanishing between electrons and muons, since they are distinguishable, but Coulomb integrals are always nonzero, regardless of which particles they refer too. Due to the heavy mass of the muons, the Born-Oppenheimer approximation becomes less valid, especially for the lighter nuclei, which cannot be assumed infinitely heavier than the muons. To account for this, OpenMolcas allows including the so-called finite nuclear

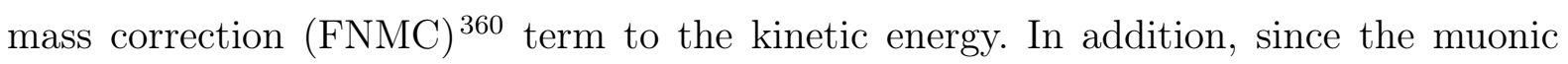
density is much more concentrated close to the nucleus, the muonic energy levels are much more sensitive to the nuclear size and charge distribution, and a point-like nuclear model is expected to be inaccurate. In OpenMolcas finite nuclear size effects can be included through a Gaussian charge distribution. Finally, the description of muons requires the use of specialized basis sets, with exponents much larger than those typically used for electronic basis functions. Such a basis set has been recently developed for elements from $\mathrm{H}$ to $\mathrm{Ar}$ and $\mathrm{Cu} \cdot \frac{359}{3 n i t i a l}$ calculations for atoms and molecules where one electron 
was replaced with one muon showed satisfactory agreement with experimentally available data, suggested a mislabeling of one of the peaks for $\mathrm{Cu}$ and allowed the estimation of the sensitivity of muonic X-ray energies to chemical environment.

\section{Summary}

OpenMolcas - an open-source community development platform - is based on the source code of the MOLCAS package. The program suite is now also a fully operational user's alternative. This move has been done under the leadership of the (Open)Molcas developers to promote the use and the development of the multiconfigurational wave function paradigm. The report includes technical details of the development platform and also a large number novel implementations. For the first part it was described how the OpenMolcas code is developed and managed. This arrangement will allow easier contributions from new developers and a more direct interaction with other software, as already exemplified by some of the interfaces reported in this work. For the second part it is reported an array of new options in OpenMolcas, which include new techniques in wave function models, tools for molecular dynamics and potential energy surface exploration, methods for computing spectroscopic and magnetic properties, utilities for graphical representation of results, and other additional new features. These items are summarized below.

The range of applicability of the CASSCF procedure has been extended by a number of new implementations and interfaces. The report describes in some detail the Stochastic-CASSCF approach, two DMRG options - the OpenMolcas-CheMPS2 and the OpenMolcas-QCMAQUIS interfaces - and new features available for the MC-PDFT method. Furthermore, new tools for exploration of the electronic PES and various types of MD have been implemented. More specifically, we report on availability of analytical nonadiabatic coupling vectors and associated optimization and characterization of conical intersections, on new options for the DYNAMIX module, and on two interfaces for molecular dynamics - SHARC for semiclassical dynamics and Quantics for on-the-fly quantum dynamics. A number of options and features have been implemented to improve the ca- 
pacity of OpenMolcas to address issues associated with different types of spectroscopy. These options include the use of the exact semiclassical operator for the light-matter interaction, the treatment of core holes, the generation of Dyson orbitals, the simulations of MCD spectra, the POLY_ANISO module for the simulation of magnetic properties of polynuclear complexes, and the use of OpenMolcas in association with ultra-accurate simulations. The OpenMolcas package has additionally been supplemented with a list of new tools for post-calculation analysis. These include LUSCUS - a general purpose GUI, a stand-alone grid generator SAGIT, the orbital visualizer Pegamoid, the generation of natural transition orbitals in the RASSI module, and the WFA module to analyze excited-state simulations. Finally, some additional features were described, such as the ANO-XS basis set, a multiscale option using frozen-density embedding techniques, implementation of higher point groups through the libMSYM library, and options to handle both the negatively charged muons and electrons at the same time.

To conclude, we have reported on the new development and application environment of the open-source quantum chemical electron structure and molecular dynamics simulation package OpenMolcas. This report described a robust software development environment. This and the documentation of a large array of new developments should stimulate more developers to join the OpenMolcas community, in particular those interested in multiconfigurational electron structure theory, and various types of molecular dynamics and spectroscopies. Moreover, to the reader, and potential user, we hope that the case has been made that OpenMolcas - a free-of-charge tool - can provide a strong and dynamic platform for state-of-the-art simulations. The user can virtually get access to new options and features as soon as the developer deems them stable for use in production calculations. Looking towards the future and new development one would in particular look forward to a robust implementation of a QM/MM interface, a better platform infrastructure to facilitate efficient massively parallel simulations, integral-direct RI techniques, and the use of new technologies, such as machine learning, to accelerate current procedures but also open for new ways in which ab initio data are used. 


\section{Acknowledgement}

K.P. and Q.M.P. thank funding from the Flemish Science Foundation (FWO) and KU Leuven Postdoctoral mandates (PDM/16/112). The computational resources and services used in DMRG-CASPT2 calculations were provided by the VSC (Flemish Supercomputer Center), funded by the Hercules Foundation and the Flemish Government-department EWI. The Zurich group was supported by the Schweizerischer Nationalfonds (Proj. no. 200021_182400). L.F. acknowledges the Austrian Science Fund FWF for a Schrödinger fellowship (Project no. J 3935). J.A. acknowledges support from the U.S. Department of Energy, Office of Basic Energy Sciences, Heavy Element Chemistry program, under grant DE-SC0001136. The work at the University of Minnesota was supported by the Air Force Office of Scientific Research under grant no. FA9550-16-1-0134 and by the National Science Foundation under grant no. CHE-1746186. M.G.D., L.K.S., M.V., M.L., M.G., E.K. and R.L. acknowledge financial support from the Stiftelsen Olle Engqvist Byggmästare, the Knut and Alice Wallenberg Foundation (grant no. KAW-2013.0020), and the Swedish Research Council (2012-3924), and thank Trond Saue for useful discussions. The project R-143-000-A65-133 of the National University of Singapore is gratefully acknowledged. The computational work for the ANISO section was fully performed on resources of the National Supercomputing Centre, Singapore (https://www.nscc.sg). R.L. and I.F.G. acknowledges funding from the Swedish Research Council (grant 2016-03398). L.Go., S.M., P.M. and M.Op. thank the University of Vienna for generous support and all the past SHARC developers. A.G. thanks funding from the European Union's Horizon 2020 research and innovation programme under the Marie Sklodowska-Curie Grant Agreement no. 658173. The Spanish MINECO grant CTQ2016-80600-P is acknowledged. The ERC Starting Grant "PhotoMutant" is acknowledged. S.M., F.P. and L.Go. gratefully acknowledge funding from the Austrian Science Fund (FWF) within project I2883. M.Ol. is grateful for grants NSF CHE-CLP-1710191, NIH GM126627 01, a USIAS 2015 fellowship and a MIUR Department of Excellence grant. S.I.B. acknowledges financial support from the Deutsche Forschungsgemeinschaft, grant No. BO 4915/1-1. J.N. acknowledges financial support from the Swedish Research Council (contract VR 2015-03956) and the 
Helmholtz Virtual Institute VI419 "Dynamic Pathways in Multidimensional Landscapes". A.Z., F.A. and T.A.W. acknowledge support from Fonds National Suisse (FNS) under grant 200020-172532.

\section{Supporting Information Available}

Example inputs for Stochastic-CASSCF, DMRG-CASPT2, DMRG-SCF, MPSSI, DMRG-NEVPT2, MC-PDFT, conical intersections, SHARC interface, light-matter interaction, Dyson orbitals, orbital properties, binatural orbitals, WFA, and FDET. Computational details for the DMRG-MRPT2 examples discussed in the text, discussion on convergence and performance for the Fe(II)-porphyrin calculation with OpenMolcas-CheMPS2. This material is available free of charge via the Internet at http://pubs.acs.org/. 


\section{References}

(1) Free Software Foundation, GNU Lesser General Public License, version 2.1. https: //www.gnu.org/licenses/old-licenses/lgpl-2.1.html.

(2) Roos, B. O.; Karlström, G.; Malmqvist, P.-Å.; Sadlej, A. J.; Widmark, P.-O. MOLCAS: A General Purpose Quantum Chemistry Program System for Correlated Wavefunctions. In Modern Techniques in Computational Chemistry: MOTECC91; Clementi, E., Ed.; ESCOM Science Publishers, 1991; Chapter 10, pp 435-454.

(3) Andersson, K.; Fülscher, M. P.; Lindh, R.; Malmqvist, P.-Å.; Olsen, J.; Roos, B. O.; Widmark, P.-O. MOLCAS: A general purpose quantum chemistry program system for correlated wavefunctions. In Methods and Techniques in Computational Chemistry: METECC-94; Clementi, E., Ed.; STEF, 1994; Chapter 7, pp 275-324.

(4) Karlström, G.; Lindh, R.; Malmqvist, P.-Å.; Roos, B. O.; Ryde, U.; Veryazov, V.; Widmark, P.-O.; Cossi, M.; Schimmelpfennig, B.; Neogrady, P.; Seijo, L. MOLCAS: a program package for computational chemistry. Comput. Mater. Sci. 2003, 28, $222-239$.

(5) Veryazov, V.; Widmark, P.-O.; Serrano-Andrés, L.; Lindh, R.; Roos, B. O. 2MOLCAS as a development platform for quantum chemistry software. Int. J. Quantum Chem. 2004, 100, 626-635.

(6) Aquilante, F.; De Vico, L.; Ferré, N.; Ghigo, G.; Malmqvist, P.-Å.; Neogrády, P.; Pedersen, T. B.; Pitoňák, M.; Reiher, M.; Roos, B. O.; Serrano-Andrés, L.; Urban, M.; Veryazov, V.; Lindh, R. MOLCAS 7: The Next Generation. J. Comput. Chem. 2010, 31, 224-247.

(7) Aquilante, F.; Pedersen, T. B.; Veryazov, V.; Lindh, R. MOLCAS-a software for multiconfigurational quantum chemistry calculations. Wiley Interdiscip. Rev.: Comput. Mol. Sci. 2013, 3, 143-149.

(8) Aquilante, F.; Autschbach, J.; Carlson, R. K.; Chibotaru, L. F.; Delcey, M. G.; 
De Vico, L.; Fdez. Galván, I.; Ferré, N.; Frutos, L. M.; Gagliardi, L.; Garavelli, M.; Giussani, A.; Hoyer, C. E.; Li Manni, G.; Lischka, H.; Ma, D.; Malmqvist, P. A.; Müller, T.; Nenov, A.; Olivucci, M.; Pedersen, T. B.; Peng, D.; Plasser, F.; Pritchard, B.; Reiher, M.; Rivalta, I.; Schapiro, I.; Segarra-Martí, J.; Stenrup, M.; Truhlar, D. G.; Ungur, L.; Valentini, A.; Vancoillie, S.; Veryazov, V.; Vysotskiy, V. P.; Weingart, O.; Zapata, F.; Lindh, R. MOLCAS 8: New capabilities for multiconfigurational quantum chemical calculations across the periodic table. J. Comput. Chem. 2015, 37, 506-541.

(9) Lotrich, V.; Flocke, N.; Ponton, M.; Yau, A. D.; Perera, A.; Deumens, E.; Bartlett, R. J. Parallel implementation of electronic structure energy, gradient, and Hessian calculations. J. Chem. Phys. 2008, 128, 194104.

(10) te Velde, G.; Bickelhaupt, F. M.; Baerends, E. J.; Fonseca Guerra, C.; van Gisbergen, S. J. A.; Snijders, J. G.; Ziegler, T. Chemistry with ADF. J. Comput. Chem. 2001, 22, 931-967.

(11) Shiozaki, T. BAGEL: Brilliantly Advanced General Electronic-structure Library. Wiley Interdiscip. Rev.: Comput. Mol. Sci. 2017, 8, e1331.

(12) Genovese, L.; Videau, B.; Ospici, M.; Deutsch, T.; Goedecker, S.; Méhaut, J.-F. Daubechies wavelets for high performance electronic structure calculations: The BigDFT project. C. R. Méc. 2011, 339, 149-164.

(13) Stanton, J. F.; Gauss, J.; Cheng, L.; Harding, M. E.; Matthews, D. A.; Szalay, P. G. CFOUR, Coupled-Cluster techniques for Computational Chemistry. http://cfour.de.

(14) Lischka, H.; Müller, T.; Szalay, P. G.; Shavitt, I.; Pitzer, R. M.; Shepard, R. Columbus-A program system for advanced multireference theory calculations. $W i$ ley Interdiscip. Rev.: Comput. Mol. Sci. 2011, 1, 191-199.

(15) Aidas, K.; Angeli, C.; Bak, K. L.; Bakken, V.; Bast, R.; Boman, L.; Christiansen, O.; Cimiraglia, R.; Coriani, S.; Dahle, P.; Dalskov, E. K.; Ekström, U.; 
Enevoldsen, T.; Eriksen, J. J.; Ettenhuber, P.; Fernández, B.; Ferrighi, L.; Fliegl, H.; Frediani, L.; Hald, K.; Halkier, A.; Hättig, C.; Heiberg, H.; Helgaker, T.; Hennum, A. C.; Hettema, H.; Hjertenaes, E.; Høst, S.; Høyvik, I.-M.; Iozzi, M. F.; Jansík, B.; Jensen, H. J. Aa.; Jonsson, D.; Jørgensen, P.; Kauczor, J.; Kirpekar, S.; Kjaergaard, T.; Klopper, W.; Knecht, S.; Kobayashi, R.; Koch, H.; Kongsted, J.; Krapp, A.; Kristensen, K.; Ligabue, A.; Lutnaes, O. B.; Melo, J. I.; Mikkelsen, K. V.; Myhre, R. H.; Neiss, C.; Nielsen, C. B.; Norman, P.; Olsen, J.; Olsen, J. M. H.; Osted, A.; Packer, M. J.; Pawlowski, F.; Pedersen, T. B.; Provasi, P. F.; Reine, S.; Rinkevicius, Z.; Ruden, T. A.; Ruud, K.; Rybkin, V. V.; Sałek, P.; Samson, C. C. M.; Sánchez de Merás, A.; Saue, T.; Sauer, S. P. A.; Schimmelpfennig, B.; Sneskov, K.; Steindal, A. H.; Sylvester-Hvid, K. O.; Taylor, P. R.; Teale, A. M.; Tellgren, E. I.; Tew, D. P.; Thorvaldsen, A. J.; Thøgersen, L.; Vahtras, O.; Watson, M. A.; Wilson, D. J. D.; Ziolkowski, M.; Ågren, H. The Dalton quantum chemistry program system. Wiley Interdiscip. Rev.: Comput. Mol. Sci. 2013, 4, 269-284.

(16) Koster, A. M.; Geudtner, G.; Alvarez-Ibarra, A.; Calaminici, P.; Casida, M. E.; Carmona-Espindola, J.; Dominguez, V. D.; Flores-Moreno, R.; Gamboa, G. U.; Goursot, A.; Heine, T.; Ipatov, A.; de la Lande, A.; Janetzko, F.; del Campo, J. M.; Mejia-Rodriguez, D.; Reveles, J. U.; Vasquez-Perez, J.; Vela, A.; ZunigaGutierrez, B.; Salahub, D. R. deMon2k, A software package for density functional theory (DFT) calculations. 2018; http://www.demon-software.com, Cinvestav, Mexico-City.

(17) Saue, T.; Visscher, L.; Jensen, H. J. Aa.; Bast, R. DIRAC, a relativistic ab initio electronic structure program. http://www.diracprogram.org.

(18) Granovsky, A. A. Firefly QC package. http://classic.chem.msu.su/gran/ gamess.

(19) Gordon, M. S.; Schmidt, M. W. Advances in electronic structure theory: GAMESS 
a decade later. In Theory and Applications of Computational Chemistry; Elsevier, 2005; Chapter 41, pp 1167-1189.

(20) Guest, M. F.; Bush, I. J.; Van Dam, H. J. J.; Sherwood, P.; Thomas, J. M. H.; Van Lenthe, J. H.; Havenith, R. W. A.; Kendrick, J. The GAMESS-UK electronic structure package: algorithms, developments and applications. Mol. Phys. 2005, 103, 719-747.

(21) Frisch, M. J.; Trucks, G. W.; Schlegel, H. B.; Scuseria, G. E.; Robb, M. A.; Cheeseman, J. R.; Scalmani, G.; Barone, V.; Petersson, G. A.; Nakatsuji, H.; Li, X.; Caricato, M.; Marenich, A. V.; Bloino, J.; Janesko, B. G.; Gomperts, R.; Mennucci, B.; Hratchian, H. P.; Ortiz, J. V.; Izmaylov, A. F.; Sonnenberg, J. L.; WilliamsYoung, D.; Ding, F.; Lipparini, F.; Egidi, F.; Goings, J.; Peng, B.; Petrone, A.; Henderson, T.; Ranasinghe, D.; Zakrzewski, V. G.; Gao, J.; Rega, N.; Zheng, G.; Liang, W.; Hada, M.; Ehara, M.; Toyota, K.; Fukuda, R.; Hasegawa, J.; Ishida, M.; Nakajima, T.; Honda, Y.; Kitao, O.; Nakai, H.; Vreven, T.; Throssell, K.; Montgomery, J. A., Jr.; Peralta, J. E.; Ogliaro, F.; Bearpark, M. J.; Heyd, J. J.; Brothers, E. N.; Kudin, K. N.; Staroverov, V. N.; Keith, T. A.; Kobayashi, R.; Normand, J.; Raghavachari, K.; Rendell, A. P.; Burant, J. C.; Iyengar, S. S.; Tomasi, J.; Cossi, M.; Millam, J. M.; Klene, M.; Adamo, C.; Cammi, R.; Ochterski, J. W.; Martin, R. L.; Morokuma, K.; Farkas, O.; Foresman, J. B.; Fox, D. J. Gaussian 16. 2016; Gaussian Inc. Wallingford CT.

(22) Nakamura, H.; Xidos, J. D.; Chamberlin, A. C.; Kelly, C. P.; Valero, R.; Yang, K. R.; Thompson, J. D.; Li, J.; Hawkins, G. D.; Zhu, T.; Lynch, B. J.; Volobuev, Y.; Rinaldi, D.; Liotard, D. A.; Cramer, C. J.; Truhlar, D. G. HONDOPLUS-v.5.2, based on HONDO v.99.6. 2013; https://t1. chem. umn.edu/ hondoplus, University of Minnesota, Minneapolis.

(23) Bochevarov, A. D.; Harder, E.; Hughes, T. F.; Greenwood, J. R.; Braden, D. A.; Philipp, D. M.; Rinaldo, D.; Halls, M. D.; Zhang, J.; Friesner, R. A. Jaguar: A 
high-performance quantum chemistry software program with strengths in life and materials sciences. Int. J. Quantum Chem. 2013, 113, 2110-2142.

(24) Werner, H.-J.; Knowles, P. J.; Knizia, G.; Manby, F. R.; Schütz, M. Molpro: a general-purpose quantum chemistry program package. Wiley Interdiscip. Rev.: Comput. Mol. Sci. 2011, 2, 242-253.

(25) Kállay, M.; Nagy, P. R.; Rolik, Z.; Mester, D.; Samu, G.; Csontos, J.; Csóka, J.; Szabó, B. P.; Gyevi-Nagy, L.; Ladjánszki, I.; Szegedy, L.; Ladóczki, B.; Petrov, K.; Farkas, M.; Mezei, P. D.; Hégely, B. MRCC, a quantum chemical program suite. https://www.mrcc.hu

(26) Valiev, M.; Bylaska, E. J.; Govind, N.; Kowalski, K.; Straatsma, T. P.; Van Dam, H. J. J.; Wang, D.; Nieplocha, J.; Apra, E.; Windus, T. L.; de Jong, W. A. NWChem: A comprehensive and scalable open-source solution for large scale molecular simulations. Comput. Phys. Commun. 2010, 181, 1477-1489.

(27) Pederson, M. R.; Jackson, K. A.; Porezag, D. V. NRMOL, Naval Research Laboratory Molecular Orbital Library. https://quantum.utep.edu/new_nrlmol/ nrlmoldoc_main.html.

(28) Neese, F. Software update: the ORCA program system, version 4.0. Wiley Interdiscip. Rev.: Comput. Mol. Sci. 2018, 8, e1327.

(29) Baker, J.; Wolinski, K.; Malagoli, M.; Kinghorn, D.; Wolinski, P.; Magyarfalvi, G.; Saebo, S.; Janowski, T.; Pulay, P. Quantum chemistry in parallel with PQS. J. Comput. Chem. 2009, 30, 317-335.

(30) Parrish, R. M.; Burns, L. A.; Smith, D. G. A.; Simmonett, A. C.; DePrince, A. E.; Hohenstein, E. G.; Bozkaya, U.; Sokolov, A. Yu.; Di Remigio, R.; Richard, R. M.; Gonthier, J. F.; James, A. M.; McAlexander, H. R.; Kumar, A.; Saitow, M.; Wang, X.; Pritchard, B. P.; Verma, P.; Schaefer, H. F.; Patkowski, K.; King, R. A.; Valeev, E. F.; Evangelista, F. A.; Turney, J. M.; Crawford, T. D.; Sherrill, C. D. 
Psi4 1.1: An Open-Source Electronic Structure Program Emphasizing Automation, Advanced Libraries, and Interoperability. J. Chem. Theory Comput. 2017, 13, 3185-3197.

(31) Sun, Q.; Berkelbach, T. C.; Blunt, N. S.; Booth, G. H.; Guo, S.; Li, Z.; Liu, J.; McClain, J. D.; Sayfutyarova, E. R.; Sharma, S.; Wouters, S.; Chan, G. K.-L. PySCF: the Python-based simulations of chemistry framework. Wiley Interdiscip. Rev.: Comput. Mol. Sci. 2017, 8, e1340.

(32) Shao, Y.; Gan, Z.; Epifanovsky, E.; Gilbert, A. T. B.; Wormit, M.; Kussmann, J.; Lange, A. W.; Behn, A.; Deng, J.; Feng, X.; Ghosh, D.; Goldey, M.; Horn, P. R.; Jacobson, L. D.; Kaliman, I.; Khaliullin, R. Z.; Kuś, T.; Landau, A.; Liu, J.; Proynov, E. I.; Rhee, Y. M.; Richard, R. M.; Rohrdanz, M. A.; Steele, R. P.; Sundstrom, E. J.; Woodcock, H. L.; Zimmerman, P. M.; Zuev, D.; Albrecht, B.; Alguire, E.; Austin, B.; Beran, G. J. O.; Bernard, Y. A.; Berquist, E.; Brandhorst, K.; Bravaya, K. B.; Brown, S. T.; Casanova, D.; Chang, C.-M.; Chen, Y.; Chien, S. H.; Closser, K. D.; Crittenden, D. L.; Diedenhofen, M.; DiStasio, R. A.; Do, H.; Dutoi, A. D.; Edgar, R. G.; Fatehi, S.; Fusti-Molnar, L.; Ghysels, A.; GolubevaZadorozhnaya, A.; Gomes, J.; Hanson-Heine, M. W. D.; Harbach, P. H. P.; Hauser, A. W.; Hohenstein, E. G.; Holden, Z. C.; Jagau, T.-C.; Ji, H.; Kaduk, B.; Khistyaev, K.; Kim, J.; Kim, J.; King, R. A.; Klunzinger, P.; Kosenkov, D.; Kowalczyk, T.; Krauter, C. M.; Lao, K. U.; Laurent, A. D.; Lawler, K. V.; Levchenko, S. V.; Lin, C. Y.; Liu, F.; Livshits, E.; Lochan, R. C.; Luenser, A.; Manohar, P.; Manzer, S. F.; Mao, S.-P.; Mardirossian, N.; Marenich, A. V.; Maurer, S. A.; Mayhall, N. J.; Neuscamman, E.; Oana, C. M.; Olivares-Amaya, R.; O’Neill, D. P.; Parkhill, J. A.; Perrine, T. M.; Peverati, R.; Prociuk, A.; Rehn, D. R.; Rosta, E.; Russ, N. J.; Sharada, S. M.; Sharma, S.; Small, D. W.; Sodt, A.; Stein, T.; Stück, D.; Su, Y.-C.; Thom, A. J. W.; Tsuchimochi, T.; Vanovschi, V.; Vogt, L.; Vydrov, O.; Wang, T.; Watson, M. A.; Wenzel, J.; White, A.; Williams, C. F.; Yang, J.; Yeganeh, S.; Yost, S. R.; You, Z.-Q.; Zhang, I. Y.; Zhang, X.; Zhao, Y.; Brooks, B. R.; Chan, G. K. L.; Chipman, D. M.; Cramer, C. J.; Goddard, W. A.; 
Gordon, M. S.; Hehre, W. J.; Klamt, A.; Schaefer, H. F.; Schmidt, M. W.; Sherrill, C. D.; Truhlar, D. G.; Warshel, A.; Xu, X.; Aspuru-Guzik, A.; Baer, R.; Bell, A. T.; Besley, N. A.; Chai, J.-D.; Dreuw, A.; Dunietz, B. D.; Furlani, T. R.; Gwaltney, S. R.; Hsu, C.-P.; Jung, Y.; Kong, J.; Lambrecht, D. S.; Liang, W.; Ochsenfeld, C.; Rassolov, V. A.; Slipchenko, L. V.; Subotnik, J. E.; Van Voorhis, T.; Herbert, J. M.; Krylov, A. I.; Gill, P. M. W.; Head-Gordon, M. Advances in molecular quantum chemistry contained in the Q-Chem 4 program package. Mol. Phys. 2014, 113, 184-215.

(33) TeraChem. http://www petachem.com/products.html, PetaChem, LLC, Los Altos Hills, CA.

(34) Furche, F.; Ahlrichs, R.; Hättig, C.; Klopper, W.; Sierka, M.; Weigend, F. Turbomole. Wiley Interdiscip. Rev.: Comput. Mol. Sci. 2013, 4, 91-100.

(35) Pirhadi, S.; Sunseri, J.; Koes, D. R. Open source molecular modeling. J. Mol. Graphics Modell. 2016, 69, 127-143.

(36) git, a free and open source distributed version control system. https://www. git-scm.com.

(37) CMake, an extensible, open-source system that manages the build process. https: //cmake.org.

(38) GitLab, a git-based fully featured platform for software development. https:// about.gitlab.com

(39) HDF5, high-performance data management and storage suite. https://www. hdfgroup.org/

(40) Čížek, J. On the Correlation Problem in Atomic and Molecular Systems. Calculation of Wavefunction Components in Ursell-Type Expansion Using Quantum-Field Theoretical Methods. J. Chem. Phys. 1966, 45, 4256-4266. 
(41) Purvis, G. D.; Bartlett, R. J. A full coupled-cluster singles and doubles model: The inclusion of disconnected triples. J. Chem. Phys. 1982, 76, 1910-1918.

(42) Raghavachari, K.; Trucks, G. W. Highly correlated systems. Excitation energies of first row transition metals Sc-Cu. J. Chem. Phys. 1989, 91, 1062-1065.

(43) Hartree, D. R. The Wave Mechanics of an Atom with a Non-Coulomb Central Field. Part I. Theory and Methods. Math. Proc. Cambridge Philos. Soc. 1928, 24, 89.

(44) Fock, V. Näherungsmethode zur Lösung des quantenmechanischen Mehrkörperproblems. Z. Phys. 1930, 61, 126-148.

(45) Roothaan, C. C. J. New Developments in Molecular Orbital Theory. Rev. Mod. Phys. 1951, 23, 69-89.

(46) Roothaan, C. C. J. Self-Consistent Field Theory for Open Shells of Electronic Systems. Rev. Mod. Phys. 1960, 32, 179-185.

(47) Roos, B. O.; Taylor, P. R.; Siegbahn, P. E. M. A complete active space SCF method (CASSCF) using a density matrix formulated super-CI approach. Chem. Phys. 1980, 48, 157-173.

(48) Roos, B. O. The complete active space SCF method in a fock-matrix-based superCI formulation. Int. J. Quantum Chem. 1980, 18, 175-189.

(49) Siegbahn, P. E. M.; Almlöf, J.; Heiberg, A.; Roos, B. O. The complete active space SCF (CASSCF) method in a Newton-Raphson formulation with application to the HNO molecule. J. Chem. Phys. 1981, 74, 2384-2396.

(50) Siegbahn, P.; Heiberg, A.; Roos, B.; Levy, B. A Comparison of the Super-CI and the Newton-Raphson Scheme in the Complete Active Space SCF Method. Phys. Status Solidi B 1980, 21, 323-327.

(51) Kohn, W.; Sham, L. J. Self-Consistent Equations Including Exchange and Correlation Effects. Phys. Rev. 1965, 140, A1133-A1138. 
(52) Peverati, R.; Truhlar, D. G. Quest for a universal density functional: the accuracy of density functionals across a broad spectrum of databases in chemistry and physics. Philos. Trans. R. Soc. A 2014, 372, 20120476.

(53) Yu, H. S.; He, X.; Li, S. L.; Truhlar, D. G. MN15: A Kohn-Sham global-hybrid exchange-correlation density functional with broad accuracy for multi-reference and single-reference systems and noncovalent interactions. Chem. Sci. 2016, 7, $5032-5051$.

(54) Li Manni, G.; Carlson, R. K.; Luo, S.; Ma, D.; Olsen, J.; Truhlar, D. G.; Gagliardi, L. Multiconfiguration Pair-Density Functional Theory. J. Chem. Theory Comput. 2014, 10, 3669-3680.

(55) Olsen, J.; Roos, B. O.; Jørgensen, P.; Jensen, H. J. Aa. Determinant based configuration interaction algorithms for complete and restricted configuration interaction spaces. J. Chem. Phys. 1988, 89, 2185-2192.

(56) Malmqvist, P. A.; Rendell, A.; Roos, B. O. The restricted active space selfconsistent-field method, implemented with a split graph unitary group approach. J. Phys. Chem. 1990, 94, 5477-5482.

(57) Ma, D.; Li Manni, G.; Gagliardi, L. The generalized active space concept in multiconfigurational self-consistent field methods. J. Chem. Phys. 2011, 135, 044128.

(58) Li Manni, G.; Ma, D.; Aquilante, F.; Olsen, J.; Gagliardi, L. SplitGAS Method for Strong Correlation and the Challenging Case of $\mathrm{Cr}_{2}$. J. Chem. Theory Comput. 2013, 9, 3375-3384.

(59) Carlson, R. K.; Li Manni, G.; Sonnenberger, A. L.; Truhlar, D. G.; Gagliardi, L. Multiconfiguration Pair-Density Functional Theory: Barrier Heights and Main Group and Transition Metal Energetics. J. Chem. Theory Comput. 2015, 11, 8290.

(60) Carlson, R. K.; Truhlar, D. G.; Gagliardi, L. Multiconfiguration Pair-Density Functional Theory: A Fully Translated Gradient Approximation and Its Performance 
for Transition Metal Dimers and the Spectroscopy of $\mathrm{Re}_{2} \mathrm{Cl}_{8}^{2-}$. J. Chem. Theory Comput. 2015, 11, 4077-4085.

(61) Gagliardi, L.; Truhlar, D. G.; Li Manni, G.; Carlson, R. K.; Hoyer, C. E.; Bao, J. L. Multiconfiguration Pair-Density Functional Theory: A New Way To Treat Strongly Correlated Systems. Acc. Chem. Res. 2017, 50, 66-73.

(62) Andersson, K.; Malmqvist, P. Å.; Roos, B. O.; Sadlej, A. J.; Wolinski, K. Secondorder perturbation theory with a CASSCF reference function. J. Phys. Chem. $1990,94,5483-5488$.

(63) Andersson, K.; Malmqvist, P.-A.; Roos, B. O. Second-order perturbation theory with a complete active space self-consistent field reference function. J. Chem. Phys. 1992, 96, 1218-1226.

(64) Malmqvist, P. A.; Pierloot, K.; Moughal Shahi, A. R.; Cramer, C. J.; Gagliardi, L. The restricted active space followed by second-order perturbation theory method: Theory and application to the study of $\mathrm{CuO}_{2}$ and $\mathrm{Cu}_{2} \mathrm{O}_{2}$ systems. J. Chem. Phys. 2008, 128, 204109.

(65) Angeli, C.; Cimiraglia, R.; Evangelisti, S.; Leininger, T.; Malrieu, J.-P. Introduction of $n$-electron valence states for multireference perturbation theory. J. Chem. Phys. 2001, 114, 10252-10264.

(66) Angeli, C.; Cimiraglia, R.; Malrieu, J.-P. N-electron valence state perturbation theory: a fast implementation of the strongly contracted variant. Chem. Phys. Lett. 2001, 350, 297-305.

(67) Angeli, C.; Cimiraglia, R.; Malrieu, J.-P. n-electron valence state perturbation theory: A spinless formulation and an efficient implementation of the strongly contracted and of the partially contracted variants. J. Chem. Phys. 2002, 117, 9138-9153. 
(68) Angeli, C.; Borini, S.; Cestari, M.; Cimiraglia, R. A quasidegenerate formulation of the second order $n$-electron valence state perturbation theory approach. J. Chem. Phys. 2004, 121, 4043-4049.

(69) Angeli, C.; Borini, S.; Cavallini, A.; Cestari, M.; Cimiraglia, R.; Ferrighi, L.; Sparta, M. Developments in the $n$-electron valence state perturbation theory. Int. J. Quantum Chem. 2006, 106, 686-691.

(70) Angeli, C.; Cimiraglia, R.; Malrieu, J.-P. A Simple Approximate Perturbation Approach to Quasi-degenerate Systems. Theor. Chem. Acc. 2006, 116, 434-439.

(71) Angeli, C.; Pastore, M.; Cimiraglia, R. New perspectives in multireference perturbation theory: the $n$-electron valence state approach. Theor. Chem. Acc. 2007, $117,743-754$.

(72) Sharma, P.; Truhlar, D. G.; Gagliardi, L. Active Space Dependence in Multiconfiguration Pair-Density Functional Theory. J. Chem. Theory Comput. 2018, 14, 660-669.

(73) Li Manni, G.; Smart, S. D.; Alavi, A. Combining the Complete Active Space Self-Consistent Field Method and the Full Configuration Interaction Quantum Monte Carlo within a Super-CI Framework, with Application to Challenging MetalPorphyrins. J. Chem. Theory Comput. 2016, 12, 1245-1258.

(74) Li Manni, G.; Alavi, A. Understanding the Mechanism Stabilizing Intermediate Spin States in Fe(II)-Porphyrin. J. Phys. Chem. A 2018, 122, 4935-4947.

(75) Li Manni, G.; Kats, D.; Tew, D. P.; Alavi, A. Role of Valence and Semicore Electron Correlation on Spin Gaps in Fe(II)-Porphyrins. J. Chem. Theory Comput. 2019, 15, 1492-1497.

(76) Bogdanov, N.; Li Manni, G.; Sharma, S.; Gunnarsson, O.; Alavi, A. New superexchange paths due to breathing-enhanced hopping in corner-sharing cuprates. 2018, preprint [arXiv:1803.07026]. 
(77) Davidson, E. R. The iterative calculation of a few of the lowest eigenvalues and corresponding eigenvectors of large real-symmetric matrices. J. Comput. Phys. 1975, $17,87-94$.

(78) Roos, B. A new method for large-scale CI calculations. Chem. Phys. Lett. 1972, $15,153-159$.

(79) Booth, G. H.; Thom, A. J. W.; Alavi, A. Fermion Monte Carlo without fixed nodes: A game of life, death, and annihilation in Slater determinant space. J. Chem. Phys. 2009, 131, 054106.

(80) Cleland, D.; Booth, G. H.; Alavi, A. Communications: Survival of the fittest: Accelerating convergence in full configuration-interaction quantum Monte Carlo. J. Chem. Phys. 2010, 132, 041103.

(81) Cleland, D. M.; Booth, G. H.; Alavi, A. A study of electron affinities using the initiator approach to full configuration interaction quantum Monte Carlo. J. Chem. Phys. 2011, 134, 024112.

(82) Overy, C.; Booth, G. H.; Blunt, N. S.; Shepherd, J. J.; Cleland, D.; Alavi, A. Unbiased reduced density matrices and electronic properties from full configuration interaction quantum Monte Carlo. J. Chem. Phys. 2014, 141, 244117.

(83) Blunt, N. S.; Smart, S. D.; Kersten, J. A. F.; Spencer, J. S.; Booth, G. H.; Alavi, A. Semi-stochastic full configuration interaction quantum Monte Carlo: Developments and application. J. Chem. Phys. 2015, 142, 184107.

(84) Booth, G. H.; Smart, S. D.; Alavi, A. Linear-scaling and parallelisable algorithms for stochastic quantum chemistry. Mol. Phys. 2014, 112, 1855-1869.

(85) Booth, G. H.; Cleland, D.; Thom, A. J. W.; Alavi, A. Breaking the carbon dimer: The challenges of multiple bond dissociation with full configuration interaction quantum Monte Carlo methods. J. Chem. Phys. 2011, 135, 084104. 
(86) Blunt, N. S.; Smart, S. D.; Booth, G. H.; Alavi, A. An excited-state approach within full configuration interaction quantum Monte Carlo. J. Chem. Phys. 2015, $143,134117$.

(87) Blunt, N. S.; Alavi, A.; Booth, G. H. Krylov-Projected Quantum Monte Carlo Method. Phys. Rev. Lett. 2015, 115, 050603.

(88) Helgaker, T.; Jørgensen, P.; Olsen, J. Molecular Electronic Structure Theory; John Wiley \& Sons: Chichester, England, 2000.

(89) Aquilante, F.; Lindh, R.; Pedersen, T. B. Unbiased auxiliary basis sets for accurate two-electron integral approximations. J. Chem. Phys. 2007, 127, 114107.

(90) Aquilante, F.; Pedersen, T. B.; Lindh, R. Low-cost evaluation of the exchange Fock matrix from Cholesky and density fitting representations of the electron repulsion integrals. J. Chem. Phys. 2007, 126, 194106.

(91) Aquilante, F.; Pedersen, T. B.; Lindh, R.; Roos, B. O.; Sánchez de Merás, A.; Koch, H. Accurate ab initio density fitting for multiconfigurational self-consistent field methods. J. Chem. Phys. 2008, 129, 024113.

(92) Aquilante, F.; Gagliardi, L.; Pedersen, T. B.; Lindh, R. Atomic Cholesky decompositions: A route to unbiased auxiliary basis sets for density fitting approximation with tunable accuracy and efficiency. J. Chem. Phys. 2009, 130, 154107.

(93) Pedersen, T. B.; Aquilante, F.; Lindh, R. Density fitting with auxiliary basis sets from Cholesky decompositions. Theor. Chem. Acc. 2009, 124, 1-10.

(94) Bednorz, J. G.; Müller, K. A. Possible high $T_{c}$ superconductivity in the Ba-La-Cu-O system. Z. Phys. B: Condens. Matter 1986, 64, 189-193.

(95) Peng, Y. Y.; Dellea, G.; Minola, M.; Conni, M.; Amorese, A.; Di Castro, D.; De Luca, G. M.; Kummer, K.; Salluzzo, M.; Sun, X.; Zhou, X. J.; Balestrino, G.; 
Le Tacon, M.; Keimer, B.; Braicovich, L.; Brookes, N. B.; Ghiringhelli, G. Influence of apical oxygen on the extent of in-plane exchange interaction in cuprate superconductors. Nat. Phys. 2017, 13, 1201-1206.

(96) van Oosten, A. B.; Broer, R.; Nieuwpoort, W. C. Heisenberg exchange enhancement by orbital relaxation in cuprate compounds. Chem. Phys. Lett. 1996, 257, 207-212.

(97) Muñoz, D.; Illas, F.; de P. R. Moreira, I. Accurate Prediction of Large Antiferromagnetic Interactions in High- $T_{c} \mathrm{HgBa}_{2} \mathrm{Ca}_{n-1} \mathrm{Cu}_{n} \mathrm{O}_{2 n+2+\delta}(n=2,3)$ Superconductor Parent Compounds. Phys. Rev. Lett. 2000, 84, 1579-1582.

(98) de Graaf, C.; Sousa, C.; de P. R. Moreira, I.; Illas, F. Multiconfigurational Perturbation Theory: An Efficient Tool to Predict Magnetic Coupling Parameters in Biradicals, Molecular Complexes, and Ionic Insulators. J. Phys. Chem. A 2001, $105,11371-11378$.

(99) Calzado, C. J.; Angeli, C.; Taratiel, D.; Caballol, R.; Malrieu, J.-P. Analysis of the magnetic coupling in binuclear systems. III. The role of the ligand to metal charge transfer excitations revisited. J. Chem. Phys. 2009, 131, 044327.

(100) Fink, K.; Staemmler, V. A modified CAS-CI approach for an efficient calculation of magnetic exchange coupling constants. Mol. Phys. 2013, 111, 2594-2605.

(101) White, S. R. Density matrix formulation for quantum renormalization groups. Phys. Rev. Lett. 1992, 69, 2863-2866.

(102) White, S. R. Density-matrix algorithms for quantum renormalization groups. Phys. Rev. B 1993, 48, 10345-10356.

(103) Östlund, S.; Rommer, S. Thermodynamic Limit of Density Matrix Renormalization. Phys. Rev. Lett. 1995, 75, 3537-3540.

(104) Rommer, S.; Östlund, S. Class of ansatz wave functions for one-dimensional spin systems and their relation to the density matrix renormalization group. Phys. Rev. B 1997, 55, 2164-2181. 
(105) Vidal, G.; Latorre, J. I.; Rico, E.; Kitaev, A. Entanglement in Quantum Critical Phenomena. Phys. Rev. Lett. 2003, 90.

(106) Verstraete, F.; Porras, D.; Cirac, J. I. Density Matrix Renormalization Group and Periodic Boundary Conditions: A Quantum Information Perspective. Phys. Rev. Lett. 2004, 93.

(107) Barthel, T.; Chung, M.-C.; Schollwöck, U. Entanglement scaling in critical twodimensional fermionic and bosonic systems. Phys. Rev. A 2006, 74.

(108) Schuch, N.; Wolf, M. M.; Verstraete, F.; Cirac, J. I. Entropy Scaling and Simulability by Matrix Product States. Phys. Rev. Lett. 2008, 100 .

(109) Schollwöck, U. The density-matrix renormalization group in the age of matrix product states. Ann. Phys. (Amsterdam, Neth.) 2011, 326, 96-192.

(110) McCulloch, I. P. From density-matrix renormalization group to matrix product states. J. Stat. Mech.: Theory Exp. 2007, 2007, P10014-P10014.

(111) Keller, S.; Reiher, M. Spin-adapted matrix product states and operators. J. Chem. Phys. 2016, 144, 134101.

(112) Bekenstein, J. D. Black Holes and Entropy. Phys. Rev. D 1973, 7, 2333-2346.

(113) Srednicki, M. Entropy and area. Phys. Rev. Lett. 1993, 71, 666-669.

(114) Olivares-Amaya, R.; Hu, W.; Nakatani, N.; Sharma, S.; Yang, J.; Chan, G. K.-L. The ab-initio density matrix renormalization group in practice. J. Chem. Phys. 2015, 142, 034102.

(115) Verstraete, F.; García-Ripoll, J. J.; Cirac, J. I. Matrix Product Density Operators: Simulation of Finite-Temperature and Dissipative Systems. Phys. Rev. Lett. 2004, 93.

(116) Crosswhite, G. M.; Doherty, A. C.; Vidal, G. Applying matrix product operators to model systems with long-range interactions. Phys. Rev. B 2008, 78. 
(117) Keller, S.; Dolfi, M.; Troyer, M.; Reiher, M. An efficient matrix product operator representation of the quantum chemical Hamiltonian. J. Chem. Phys. 2015, 143, 244118.

(118) Chan, G. K.-L.; Keselman, A.; Nakatani, N.; Li, Z.; White, S. R. Matrix product operators, matrix product states, and ab initio density matrix renormalization group algorithms. J. Phys. Chem. 2016, 145, 014102.

(119) Hubig, C.; McCulloch, I. P.; Schollwöck, U. Generic construction of efficient matrix product operators. Phys. Rev. B 2017, 95.

(120) Battaglia, S.; Keller, S.; Knecht, S. Efficient Relativistic Density-Matrix Renormalization Group Implementation in a Matrix-Product Formulation. J. Chem. Theory Comput. 2018, 14, 2353-2369.

(121) Knecht, S.; Legeza, Ö.; Reiher, M. Communication: Four-component density matrix renormalization group. J. Chem. Phys. 2014, 140, 041101.

(122) Kurashige, Y.; Yanai, T. Second-order perturbation theory with a density matrix renormalization group self-consistent field reference function: Theory and application to the study of chromium dimer. J. Chem. Phys. 2011, 135, 094104.

(123) Freitag, L.; Knecht, S.; Angeli, C.; Reiher, M. Multireference Perturbation Theory with Cholesky Decomposition for the Density Matrix Renormalization Group. J. Chem. Theory Comput. 2017, 13, 451-459.

(124) Knecht, S.; Keller, S.; Autschbach, J.; Reiher, M. A Nonorthogonal StateInteraction Approach for Matrix Product State Wave Functions. J. Chem. Theory Comput. 2016, 12, 5881-5894.

(125) Wouters, S.; Poelmans, W.; Ayers, P. W.; Van Neck, D. CheMPS2: A free opensource spin-adapted implementation of the density matrix renormalization group for ab initio quantum chemistry. Comput. Phys. Commun. 2014, 185, 1501-1514. 
(126) Knecht, S.; Hedegård, E. D.; Keller, S.; Kovyrshin, A.; Ma, Y.; Muolo, A.; Stein, C. J.; Reiher, M. New Approaches for ab initio Calculations of Molecules with Strong Electron Correlation. Chimia 2016, 70, 244-251.

(127) Wouters, S.; Van Speybroeck, V.; Van Neck, D. DMRG-CASPT2 study of the longitudinal static second hyperpolarizability of all-trans polyenes. J. Chem. Phys. 2016, 145, 054120 .

(128) Freitag, L.; Ma, Y.; Baiardi, A.; Knecht, S.; Reiher, M. Analytical gradients and nonadiabatic couplings for the state-average density matrix renormalization group self-consistent field method. 2019, preprint [arXiv:1905.01558].

(129) Phung, Q. M.; Wouters, S.; Pierloot, K. Cumulant Approximated Second-Order Perturbation Theory Based on the Density Matrix Renormalization Group for Transition Metal Complexes: A Benchmark Study. J. Chem. Theory Comput. 2016, 12, 4352-4361.

(130) Nakatani, N.; Guo, S. Density matrix renormalization group (DMRG) method as a common tool for large active-space CASSCF/CASPT2 calculations. J. Chem. Phys. 2017, 146, 094102.

(131) Knowles, P. J.; Handy, N. C. A determinant based full configuration interaction program. Comput. Phys. Commun. 1989, 54, 75-83.

(132) Roos, B. O. The Complete Active Space Self-Consistent Field Method and its Applications in Electronic Structure Calculations. In Ab Initio Methods in Quantum Chemistry Part 2; Lawley, K. P., Ed.; Advances in Chemical Physics; John Wiley \& Sons, Inc., 1987; pp 399-445.

(133) Schmidt, M. W.; Gordon, M. S. The Construction and Interpretation of MCSCF Wavefunctions. Annu. Rev. Phys. Chem. 1998, 49, 233-266.

(134) Pierloot, K. Nondynamic Correlation Effects in Transition Metal Coordination Compounds. In Computational Organometallic Chemistry; Cundari, T. R., Ed.; CRC Press, 2001; pp 123-158. 
(135) Pierloot, K. The CASPT2 method in inorganic electronic spectroscopy: from ionic transition metal to covalent actinide complexes. Mol. Phys. 2003, 101, 2083-2094.

(136) Veryazov, V.; Malmqvist, P. A.; Roos, B. O. How to select active space for multiconfigurational quantum chemistry? Int. J. Quantum Chem. 2011, 111, 3329-3338.

(137) González, L.; Escudero, D.; Serrano-Andrés, L. Progress and Challenges in the Calculation of Electronic Excited States. ChemPhysChem 2012, 13, 28-51.

(138) Stein, C. J.; Reiher, M. Automated Selection of Active Orbital Spaces. J. Chem. Theory Comput. 2016, 12, 1760-1771.

(139) Legeza, Ö.; Sólyom, J. Optimizing the density-matrix renormalization group method using quantum information entropy. Phys. Rev. B 2003, 68.

(140) Rissler, J.; Noack, R. M.; White, S. R. Measuring orbital interaction using quantum information theory. Chem. Phys. 2006, 323, 519-531.

(141) Stein, C. J.; von Burg, V.; Reiher, M. The Delicate Balance of Static and Dynamic Electron Correlation. J. Chem. Theory Comput. 2016, 12, 3764-3773.

(142) Stein, C. J.; Reiher, M. Automated Identification of Relevant Frontier Orbitals for Chemical Compounds and Processes. Chimia 2017, 71, 170-176.

(143) Stein, C. J.; Reiher, M. SCINE autoCAS. 2018; https://scine.ethz.ch/ download/autocas, accessed May, 2019.

(144) Stein, C. J.; Reiher, M. autoCAS: a program for fully automated multiconfigurational calculations. 2019, preprint [arXiv:1904.00097].

(145) SCINE: Software for Chemical Interaction Networks. https://scine.ethz.ch.

(146) Stålring, J.; Bernhardsson, A.; Lindh, R. Analytical gradients of a state average MCSCF state and a state average diagnostic. Mol. Phys. 2001, 99, 103-114.

(147) Delcey, M. G.; Freitag, L.; Pedersen, T. B.; Aquilante, F.; Lindh, R.; González, L. Analytical gradients of complete active space self-consistent field energies using 
Cholesky decomposition: Geometry optimization and spin-state energetics of a ruthenium nitrosyl complex. J. Chem. Phys. 2014, 140, 174103.

(148) Delcey, M. G.; Pedersen, T. B.; Aquilante, F.; Lindh, R. Analytical gradients of the state-average complete active space self-consistent field method with density fitting. J. Chem. Phys. 2015, 143, 044110.

(149) Hu, W.; Chan, G. K.-L. Excited-State Geometry Optimization with the Density Matrix Renormalization Group, as Applied to Polyenes. J. Chem. Theory Comput. 2015, 11, 3000-3009.

(150) Ma, Y.; Knecht, S.; Reiher, M. Multiconfigurational Effects in Theoretical Resonance Raman Spectra. ChemPhysChem 2017, 18, 384-393.

(151) Veis, L.; Antalík, A.; Brabec, J.; Neese, F.; Legeza, Ö.; Pittner, J. Coupled Cluster Method with Single and Double Excitations Tailored by Matrix Product State Wave Functions. J. Phys. Chem. Lett. 2016, 7, 4072-4078.

(152) Veis, L.; Antalík, A.; Legeza, Ö.; Alavi, A.; Pittner, J. The Intricate Case of Tetramethyleneethane: A Full Configuration Interaction Quantum Monte Carlo Benchmark and Multireference Coupled Cluster Studies. J. Chem. Theory Comput. 2018, 14, 2439-2445.

(153) Faulstich, F. M.; Laestadius, A.; Kvaal, S.; Legeza, Ö.; Schneider, R. Analysis of The Coupled-Cluster Method Tailored by Tensor-Network States in Quantum Chemistry. 2018, preprint [arXiv:1802.05699].

(154) Roca-Sanjuán, D.; Aquilante, F.; Lindh, R. Multiconfiguration second-order perturbation theory approach to strong electron correlation in chemistry and photochemistry. Wiley Interdiscip. Rev.: Comput. Mol. Sci. 2011, 2, 585-603.

(155) Dyall, K. G. The choice of a zeroth-order Hamiltonian for second-order perturbation theory with a complete active space self-consistent-field reference function. J. Chem. Phys. 1995, 102, 4909-4918. 
(156) Guo, S.; Watson, M. A.; Hu, W.; Sun, Q.; Chan, G. K.-L. N-Electron Valence State Perturbation Theory Based on a Density Matrix Renormalization Group Reference Function, with Applications to the Chromium Dimer and a Trimer Model of Poly $(p$ Phenylenevinylene). J. Chem. Theory Comput. 2016, 12, 1583-1591.

(157) Meyer, W. Configuration Expansion by Means of Pseudonatural Orbitals. In Methods of Electronic Structure Theory; Springer US, 1977; pp 413-446.

(158) Siegbahn, P. E. M. Direct configuration interaction with a reference state composed of many reference configurations. Int. J. Quantum Chem. 1980, 18, 1229-1242.

(159) Werner, H.-J.; Reinsch, E.-A. The self-consistent electron pairs method for multiconfiguration reference state functions. J. Chem. Phys. 1982, 76, 3144-3156.

(160) Beebe, N. H. F.; Linderberg, J. Simplifications in the generation and transformation of two-electron integrals in molecular calculations. Int. J. Quantum Chem. 1977, 12, 683-705.

(161) Aquilante, F.; Malmqvist, P.-A.; Pedersen, T. B.; Ghosh, A.; Roos, B. O. Cholesky Decomposition-Based Multiconfiguration Second-Order Perturbation Theory (CDCASPT2): Application to the Spin-State Energetics of $\mathrm{Co}^{\text {III }}$ (diiminato)(NPh). J. Chem. Theory Comput. 2008, 4, 694-702.

(162) Aquilante, F.; Boman, L.; Boström, J.; Koch, H.; Lindh, R.; Sánchez de Merás, A.; Pedersen, T. B. Cholesky Decomposition Techniques in Electronic Structure Theory. In Linear-Scaling Techniques in Computational Chemistry and Physics; Springer Netherlands, 2011; pp 301-343.

(163) Malmqvist, P. A.; Roos, B. O.; Schimmelpfennig, B. The restricted active space (RAS) state interaction approach with spin-orbit coupling. Chem. Phys. Lett. 2002, 357, 230-240.

(164) Gendron, F.; Pritchard, B.; Bolvin, H.; Autschbach, J. Magnetic Resonance Properties of Actinyl Carbonate Complexes and Plutonyl(VI)-tris-nitrate. Inorg. Chem. 2014, 53, 8577-8592. 
(165) Pierloot, K.; Phung, Q. M.; Domingo, A. Spin State Energetics in First-Row Transition Metal Complexes: Contribution of (3s3p) Correlation and Its Description by Second-Order Perturbation Theory. J. Chem. Theory Comput. 2017, 13, 537-553.

(166) Phung, Q. M.; Feldt, M.; Harvey, J. N.; Pierloot, K. Toward Highly Accurate Spin State Energetics in First-Row Transition Metal Complexes: A Combined CASPT2/CC Approach. J. Chem. Theory Comput. 2018, 14, 2446-2455.

(167) Harding, M. E.; Metzroth, T.; Gauss, J.; Auer, A. A. Parallel Calculation of CCSD and $\operatorname{CCSD}(\mathrm{T})$ Analytic First and Second Derivatives. J. Chem. Theory Comput. 2008, 4, 64-74.

(168) Atanasov, M.; Zadrozny, J. M.; Long, J. R.; Neese, F. A theoretical analysis of chemical bonding, vibronic coupling, and magnetic anisotropy in linear iron(II) complexes with single-molecule magnet behavior. Chem. Sci. 2013, 4, 139-156.

(169) Guo, Y.; Sivalingam, K.; Valeev, E. F.; Neese, F. SparseMaps-A systematic infrastructure for reduced-scaling electronic structure methods. III. Linear-scaling multireference domain-based pair natural orbital $N$-electron valence perturbation theory. J. Chem. Phys. 2016, 144, 094111.

(170) Coughtrie, D. J.; Giereth, R.; Kats, D.; Werner, H.-J.; Köhn, A. Embedded Multireference Coupled Cluster Theory. J. Chem. Theory Comput. 2018, 14, 693-709.

(171) Fleig, T.; Olsen, J.; Marian, C. M. The generalized active space concept for the relativistic treatment of electron correlation. I. Kramers-restricted two-component configuration interaction. J. Chem. Phys. 2001, 114, 4775-4790.

(172) Schollwöck, U. The density-matrix renormalization group. Rev. Mod. Phys. 2005, rॅ, 259-315.

(173) Marti, K. H.; Ondík, I. M.; Moritz, G.; Reiher, M. Density matrix renormalization group calculations on relative energies of transition metal complexes and clusters. J. Chem. Phys. 2008, 128, 014104. 
(174) Roos, B. O. Multiconfigurational quantum chemistry. In Theory and Applications of Computational Chemistry; Elsevier, 2005; pp 725-764.

(175) Ruedenberg, K.; Cheung, L. M.; Elbert, S. T. MCSCF optimization through combined use of natural orbitals and the Brillouin-Levy-Berthier theorem. Int. J. Quantum Chem. 1979, 16, 1069-1101.

(176) Werner, H.-J.; Meyer, W. A quadratically convergent MCSCF method for the simultaneous optimization of several states. J. Chem. Phys. 1981, 74, 5794-5801.

(177) Bao, J. J.; Gagliardi, L.; Truhlar, D. G. Multiconfiguration pair-density functional theory for doublet excitation energies and excited state geometries: the excited states of CN. Phys. Chem. Chem. Phys. 2017, 19, 30089-30096.

(178) Bao, J. L.; Gagliardi, L.; Truhlar, D. G. Self-Interaction Error in Density Functional Theory: An Appraisal. J. Phys. Chem. Lett. 2018, 9, 2353-2358.

(179) Sharma, P.; Bernales, V.; Truhlar, D. G.; Gagliardi, L. Valence $\pi \pi^{*}$ Excitations in Benzene Studied by Multiconfiguration Pair-Density Functional Theory. J. Phys. Chem. Lett. 2019, 10, 75-81.

(180) Gáspár, R. Über eine Approximation des Hartree-Fockschen Potentials Durch eine Universelle Potentialfunktion. Acta Phys. Hung. 1954, 3, 263-286.

(181) Vosko, S. H.; Wilk, L.; Nusair, M. Accurate spin-dependent electron liquid correlation energies for local spin density calculations: a critical analysis. Can. J. Phys. 1980, 58, 1200-1211.

(182) Perdew, J. P.; Burke, K.; Ernzerhof, M. Generalized Gradient Approximation Made Simple. Phys. Rev. Lett. 1996, 7r, 3865-3868.

(183) Zhang, Y.; Yang, W. Comment on "Generalized Gradient Approximation Made Simple". Phys. Rev. Lett. 1998, 80, 890-890. 
(184) Hoyer, C. E.; Ghosh, S.; Truhlar, D. G.; Gagliardi, L. Multiconfiguration PairDensity Functional Theory Is as Accurate as CASPT2 for Electronic Excitation. J. Phys. Chem. Lett. 2016, 7, 586-591.

(185) Becke, A. D. Density-functional exchange-energy approximation with correct asymptotic behavior. Phys. Rev. A 1988, 38, 3098-3100.

(186) Lee, C.; Yang, W.; Parr, R. G. Development of the Colle-Salvetti correlation-energy formula into a functional of the electron density. Phys. Rev. B 1988, 37, 785-789.

(187) Handy, N. C.; Cohen, A. J. Left-right correlation energy. Mol. Phys. 2001, 99, $403-412$.

(188) Bao, J. J.; Gagliardi, L.; Truhlar, D. G. Weak Interactions in Alkaline Earth Metal Dimers by Pair-Density Functional Theory. J. Phys. Chem. Lett. 2019, 10, 799805 .

(189) Verma, P.; Truhlar, D. G. HLE16: A Local Kohn-Sham Gradient Approximation with Good Performance for Semiconductor Band Gaps and Molecular Excitation Energies. J. Phys. Chem. Lett. 2017, 8, 380-387.

(190) Verma, P.; Truhlar, D. G. HLE17: An Improved Local Exchange-Correlation Functional for Computing Semiconductor Band Gaps and Molecular Excitation Energies. J. Phys. Chem. C 2017, 121, 7144-7154.

(191) Sand, A. M.; Truhlar, D. G.; Gagliardi, L. Efficient algorithm for multiconfiguration pair-density functional theory with application to the heterolytic dissociation energy of ferrocene. J. Chem. Phys. 2017, 146, 034101.

(192) Odoh, S. O.; Li Manni, G.; Carlson, R. K.; Truhlar, D. G.; Gagliardi, L. Separatedpair approximation and separated-pair pair-density functional theory. Chem. Sci. 2016, 7, 2399-2413.

(193) Bao, J. L.; Odoh, S. O.; Gagliardi, L.; Truhlar, D. G. Predicting Bond Dissociation Energies of Transition-Metal Compounds by Multiconfiguration Pair-Density 
Functional Theory and Second-Order Perturbation Theory Based on Correlated Participating Orbitals and Separated Pairs. J. Chem. Theory Comput. 2017, 13, $616-626$.

(194) Ghosh, S.; Cramer, C. J.; Truhlar, D. G.; Gagliardi, L. Generalized-active-space pair-density functional theory: an efficient method to study large, strongly correlated, conjugated systems. Chem. Sci. 2017, 8, 2741-2750.

(195) Bao, J. L.; Sand, A.; Gagliardi, L.; Truhlar, D. G. Correlated-Participating-Orbitals Pair-Density Functional Method and Application to Multiplet Energy Splittings of Main-Group Divalent Radicals. J. Chem. Theory Comput. 2016, 12, 4274-4283.

(196) Stoneburner, S. J.; Truhlar, D. G.; Gagliardi, L. MC-PDFT can calculate singlettriplet splittings of organic diradicals. J. Chem. Phys. 2018, 148, 064108.

(197) Wilbraham, L.; Verma, P.; Truhlar, D. G.; Gagliardi, L.; Ciofini, I. Multiconfiguration Pair-Density Functional Theory Predicts Spin-State Ordering in Iron Complexes with the Same Accuracy as Complete Active Space Second-Order Perturbation Theory at a Significantly Reduced Computational Cost. J. Phys. Chem. Lett. 2017, 8, 2026-2030.

(198) Ghosh, S.; Sonnenberger, A. L.; Hoyer, C. E.; Truhlar, D. G.; Gagliardi, L. Multiconfiguration Pair-Density Functional Theory Outperforms Kohn-Sham Density Functional Theory and Multireference Perturbation Theory for Ground-State and Excited-State Charge Transfer. J. Chem. Theory Comput. 2015, 11, 3643-3649.

(199) Hoyer, C. E.; Gagliardi, L.; Truhlar, D. G. Multiconfiguration Pair-Density Functional Theory Spectral Calculations Are Stable to Adding Diffuse Basis Functions. J. Phys. Chem. Lett. 2015, 6, 4184-4188.

(200) Dong, S. S.; Gagliardi, L.; Truhlar, D. G. Excitation spectra of retinal by multiconfiguration pair-density functional theory. Phys. Chem. Chem. Phys. 2018, 20, $7265-7276$. 
(201) Bao, J. J.; Dong, S. S.; Gagliardi, L.; Truhlar, D. G. Automatic Selection of an Active Space for Calculating Electronic Excitation Spectra by MS-CASPT2 or MC-PDFT. J. Chem. Theory Comput. 2018, 14, 2017-2025.

(202) Ghosh, S.; Verma, P.; Cramer, C. J.; Gagliardi, L.; Truhlar, D. G. Combining Wave Function Methods with Density Functional Theory for Excited States. Chem. Rev. (Washington, DC, U. S.) 2018, 118, 7249-7292.

(203) Fleig, T.; Olsen, J.; Visscher, L. The generalized active space concept for the relativistic treatment of electron correlation. II. Large-scale configuration interaction implementation based on relativistic 2- and 4-spinors and its application. J. Chem. Phys. 2003, 119, 2963-2971.

(204) Tishchenko, O.; Zheng, J.; Truhlar, D. G. Multireference Model Chemistries for Thermochemical Kinetics. J. Chem. Theory Comput. 2008, 4, 1208-1219.

(205) Sand, A. M.; Hoyer, C. E.; Sharkas, K.; Kidder, K. M.; Lindh, R.; Truhlar, D. G.; Gagliardi, L. Analytic Gradients for Complete Active Space Pair-Density Functional Theory. J. Chem. Theory Comput. 2017, 14, 126-138.

(206) Sharma, P.; Bernales, V.; Knecht, S.; Truhlar, D. G.; Gagliardi, L. Density matrix renormalization group pair-density functional theory (DMRG-PDFT): singlettriplet gaps in polyacenes and polyacetylenes. Chem. Sci. 2019, 10, 1716-1723.

(207) Zhou, C.; Gagliardi, L.; Truhlar, D. G. Multiconfiguration Pair-Density Functional Theory for Iron Porphyrin with CAS, RAS, and DMRG Active Spaces. J. Phys. Chem. A 2019, 123, 3389-3394.

(208) Freitag, L.; Keller, S.; Knecht, S.; Ma, Y.; Stein, C.; Reiher, M. A quick user guide to the SCINE-QCMaquis software suite for OpenMolcas. 2018; https://scine. ethz.ch/static/download/qcmaquis_manual.pdf.

(209) Sand, A. M.; Hoyer, C. E.; Truhlar, D. G.; Gagliardi, L. State-interaction pairdensity functional theory. J. Chem. Phys. 2018, 149, 024106. 
(210) Dong, S. S.; Huang, K. B.; Gagliardi, L.; Truhlar, D. G. State-Interaction PairDensity Functional Theory Can Accurately Describe a Spiro Mixed Valence Compound. J. Phys. Chem. A 2019, 123, 2100-2106.

(211) Finley, J.; Malmqvist, P.-A.; Roos, B. O.; Serrano-Andrés, L. The multi-state CASPT2 method. Chem. Phys. Lett. 1998, 288, 299-306.

(212) Granovsky, A. A. Extended multi-configuration quasi-degenerate perturbation theory: The new approach to multi-state multi-reference perturbation theory. J. Chem. Phys. 2011, 134, 214113.

(213) Nakano, H. Quasidegenerate perturbation theory with multiconfigurational selfconsistent-field reference functions. J. Chem. Phys. 1993, 99, 7983-7992.

(214) Nakano, H. MCSCF reference quasidegenerate perturbation theory with EpsteinNesbet partitioning. Chem. Phys. Lett. 1993, 207, 372-378.

(215) Malhado, J. P.; Bearpark, M. J.; Hynes, J. T. Non-adiabatic dynamics close to conical intersections and the surface hopping perspective. Front. Chem. (Lausanne, Switz.) 2014, 2.

(216) Jasper, A. W.; Kendrick, B. K.; Mead, C. A.; Truhlar, D. G. Non-BornOppenheimer Chemistry: Potential Surfaces, Couplings, and Dynamics. In Modern Trends in Chemical Reaction Dynamics; World Scientific, 2004; pp 329-391.

(217) Vacher, M.; Bearpark, M. J.; Robb, M. A. Direct methods for non-adiabatic dynamics: connecting the single-set variational multi-configuration Gaussian (vMCG) and Ehrenfest perspectives. Theor. Chem. Acc. 2016, 135.

(218) Maeda, S.; Ohno, K.; Morokuma, K. Updated Branching Plane for Finding Conical Intersections without Coupling Derivative Vectors. J. Chem. Theory Comput. 2010, 6, 1538-1545.

(219) Fdez. Galván, I.; Delcey, M. G.; Pedersen, T. B.; Aquilante, F.; Lindh, R. Analytical State-Average Complete-Active-Space Self-Consistent Field Nonadiabatic 
Coupling Vectors: Implementation with Density-Fitted Two-Electron Integrals and Application to Conical Intersections. J. Chem. Theory Comput. 2016, 12, 36363653.

(220) Lischka, H.; Dallos, M.; Szalay, P. G.; Yarkony, D. R.; Shepard, R. Analytic evaluation of nonadiabatic coupling terms at the MR-CI level. I. Formalism. J. Chem. Phys. 2004, 120, 7322-7329.

(221) Anglada, J. M.; Bofill, J. M. A reduced-restricted-quasi-Newton-Raphson method for locating and optimizing energy crossing points between two potential energy surfaces. J. Comput. Chem. 1997, 18, 992-1003.

(222) De Vico, L.; Olivucci, M.; Lindh, R. New General Tools for Constrained Geometry Optimizations. J. Chem. Theory Comput. 2005, 1, 1029-1037.

(223) Vreven, T.; Bernardi, F.; Garavelli, M.; Olivucci, M.; Robb, M. A.; Schlegel, H. B. Ab Initio Photoisomerization Dynamics of a Simple Retinal Chromophore Model. J. Am. Chem. Soc. 1997, 119, 12687-12688.

(224) Tully, J. C. Molecular dynamics with electronic transitions. J. Chem. Phys. 1990, 93, 1061-1071.

(225) García-Iriepa, C.; Marazzi, M.; Zapata, F.; Valentini, A.; Sampedro, D.; Frutos, L. M. Chiral Hydrogen Bond Environment Providing Unidirectional Rotation in Photoactive Molecular Motors. J. Phys. Chem. Lett. 2013, 4, 1389-1396.

(226) Gozem, S.; Melaccio, F.; Valentini, A.; Filatov, M.; Huix-Rotllant, M.; Ferré, N.; Frutos, L. M.; Angeli, C.; Krylov, A. I.; Granovsky, A. A.; Lindh, R.; Olivucci, M. Shape of Multireference, Equation-of-Motion Coupled-Cluster, and Density Functional Theory Potential Energy Surfaces at a Conical Intersection. J. Chem. Theory Comput. 2014, 10, 3074-3084.

(227) Marchand, G.; Eng, J.; Schapiro, I.; Valentini, A.; Frutos, L. M.; Pieri, E.; Olivucci, M.; Léonard, J.; Gindensperger, E. Directionality of Double-Bond Pho- 
toisomerization Dynamics Induced by a Single Stereogenic Center. J. Phys. Chem. Lett. 2015, 6, 599-604.

(228) Manathunga, M.; Yang, X.; Luk, H. L.; Gozem, S.; Frutos, L. M.; Valentini, A.; Ferré, N.; Olivucci, M. Probing the Photodynamics of Rhodopsins with Reduced Retinal Chromophores. J. Chem. Theory Comput. 2016, 12, 839-850.

(229) Valentini, A.; Rivero, D.; Zapata, F.; García-Iriepa, C.; Marazzi, M.; Palmeiro, R.; Fdez. Galván, I.; Sampedro, D.; Olivucci, M.; Frutos, L. M. Optomechanical Control of Quantum Yield in Trans-Cis Ultrafast Photoisomerization of a Retinal Chromophore Model. Angew. Chem., Int. Ed. 2017, 56, 3842-3846.

(230) Gueye, M.; Manathunga, M.; Agathangelou, D.; Orozco, Y.; Paolino, M.; Fusi, S.; Haacke, S.; Olivucci, M.; Léonard, J. Engineering the vibrational coherence of vision into a synthetic molecular device. Nat. Commun. 2018, 9.

(231) García-Iriepa, C.; Sampedro, D.; Mendicuti, F.; Léonard, J.; Frutos, L. M. Photoreactivity Control Mediated by Molecular Force Probes in Stilbene. J. Phys. Chem. Lett. 2019, 10, 1063-1067.

(232) Schnedermann, C.; Yang, X.; Liebel, M.; Spillane, K. M.; Lugtenburg, J.; Fernández, I.; Valentini, A.; Schapiro, I.; Olivucci, M.; Kukura, P.; Mathies, R. A. Evidence for a vibrational phase-dependent isotope effect on the photochemistry of vision. Nat. Chem. 2018, 10, 449-455.

(233) Schapiro, I.; Ryazantsev, M. N.; Frutos, L. M.; Ferré, N.; Lindh, R.; Olivucci, M. The Ultrafast Photoisomerizations of Rhodopsin and Bathorhodopsin Are Modulated by Bond Length Alternation and HOOP Driven Electronic Effects. J. Am. Chem. Soc. 2011, 133, 3354-3364.

(234) Gozem, S.; Luk, H. L.; Schapiro, I.; Olivucci, M. Theory and Simulation of the Ultrafast Double-Bond Isomerization of Biological Chromophores. Chem. Rev. (Washington, DC, U. S.) 2017, 117, 13502-13565. 
(235) Vacher, M.; Farahani, P.; Valentini, A.; Frutos, L. M.; Karlsson, H. O.; Fdez. Galván, I.; Lindh, R. How Do Methyl Groups Enhance the Triplet Chemiexcitation Yield of Dioxetane? J. Phys. Chem. Lett. 2017, 8, 3790-3794.

(236) Richter, M.; Marquetand, P.; González-Vázquez, J.; Sola, I.; González, L. SHARC: Ab Initio Molecular Dynamics with Surface Hopping in the Adiabatic Representation Including Arbitrary Couplings. J. Chem. Theory Comput. 2011, 7, 1253-1258.

(237) Mai, S.; Marquetand, P.; González, L. A general method to describe intersystem crossing dynamics in trajectory surface hopping. Int. J. Quantum Chem. 2015, $115,1215-1231$.

(238) Mai, S.; Marquetand, P.; González, L. Nonadiabatic dynamics: The SHARC approach. Wiley Interdiscip. Rev.: Comput. Mol. Sci. 2018, 8, e1370.

(239) Mai, S.; Richter, M.; Heindl, M.; Menger, M. F. S. J.; Atkins, A.; Ruckenbauer, M.; Plasser, F.; Oppel, M.; Marquetand, P.; González, L. SHARC2.0: Surface Hopping Including Arbitrary Couplings - A Program Package for Non-Adiabatic Dynamics. 2018; https://www. sharc-md.org.

(240) SHARC GitHub repository. https://github.com/sharc-md/sharc.

(241) Granucci, G.; Persico, M.; Toniolo, A. Direct semiclassical simulation of photochemical processes with semiempirical wave functions. J. Chem. Phys. 2001, 114, $10608-10615$.

(242) Plasser, F.; Ruckenbauer, M.; Mai, S.; Oppel, M.; Marquetand, P.; González, L. Efficient and Flexible Computation of Many-Electron Wave Function Overlaps. J. Chem. Theory Comput. 2016, 12, 1207-1219.

(243) Worth, G. A.; Giri, K.; Richings, G. W.; Burghardt, I.; Beck, M. H.; Jäckle, A.; Meyer, H.-D. The QUANTICS Package, Version 1.1, University of Birmingham, Birmingham, U.K. 2015; http://chemb125.chem.ucl.ac.uk/quantics/. 
(244) Worth, G. A.; Beck, M. H.; Jäckle, A.; Meyer, H.-D. The MCTDH Package, Version 8.3. 2003; http://www.pci.uni-heildelberg.de/tc/usr/mctdh/.

(245) Burghardt, I.; Meyer, H.-D.; Cederbaum, L. S. Approaches to the approximate treatment of complex molecular systems by the multiconfiguration time-dependent Hartree method. J. Chem. Phys. 1999, 111, 2927-2939.

(246) Richings, G. W.; Polyak, I.; Spinlove, K. E.; Worth, G. A.; Burghardt, I.; Lasorne, B. Quantum dynamics simulations using Gaussian wavepackets: the vMCG method. Int. Rev. Phys. Chem. 2015, 34, 269-308.

(247) Although not implemented yet, one could read the relevant information instead from the HDF5 standard format files generated by OpenMolcas.

(248) Köppel, H.; Schubert, B. The concept of regularized diabatic states for a general conical intersection. Mol. Phys. 2006, 104, 1069-1079.

(249) Richings, G. W.; Worth, G. A. A Practical Diabatisation Scheme for Use with the Direct-Dynamics Variational Multi-Configuration Gaussian Method. J. Phys. Chem. A 2015, 119, 12457-12470.

(250) Collins, M. A.; Zhang, D. H. Application of interpolated potential energy surfaces to quantum reactive scattering. J. Chem. Phys. 1999, 111, 9924-9931.

(251) Frankcombe, T. J. Using Hessian update formulae to construct modified Shepard interpolated potential energy surfaces: Application to vibrating surface atoms. J. Chem. Phys. 2014, 140, 114108.

(252) Heß, B. A.; Marian, C. M.; Wahlgren, U.; Gropen, O. A mean-field spin-orbit method applicable to correlated wavefunctions. Chem. Phys. Lett. 1996, 251, 365371.

(253) Bernadotte, S.; Atkins, A. J.; Jacob, C. R. Origin-independent calculation of quadrupole intensities in X-ray spectroscopy. J. Chem. Phys. 2012, 137, 204106. 
(254) Guo, M.; Sørensen, L. K.; Delcey, M. G.; Pinjari, R. V.; Lundberg, M. Simulations of iron K pre-edge X-ray absorption spectra using the restricted active space method. Phys. Chem. Chem. Phys. 2016, 18, 3250-3259.

(255) Guo, M.; Källman, E.; Sørensen, L. K.; Delcey, M. G.; Pinjari, R. V.; Lundberg, M. Molecular Orbital Simulations of Metal 1s2p Resonant Inelastic X-ray Scattering. J. Phys. Chem. A 2016, 120, 5848-5855.

(256) Sørensen, L. K.; Lindh, R.; Lundberg, M. Gauge origin independence in finite basis sets and perturbation theory. Chem. Phys. Lett. 2017, 683, 536-542.

(257) Sørensen, L. K.; Guo, M.; Lindh, R.; Lundberg, M. Applications to metal K preedges of transition metal dimers illustrate the approximate origin independence for the intensities in the length representation. Mol. Phys. 2017, 115, 174-189.

(258) List, N. H.; Kauczor, J.; Saue, T.; Jensen, H. J. Aa.; Norman, P. Beyond the electric-dipole approximation: A formulation and implementation of molecular response theory for the description of absorption of electromagnetic field radiation. J. Chem. Phys. 2015, 142, 244111.

(259) Sørensen, L. K.; Kieri, E.; Srivastav, S.; Lundberg, M.; Lindh, R. Implementation of a semiclassical light-matter interaction using the Gauss-Hermite quadrature: A simple alternative to the multipole expansion. Phys. Rev. A 2019, 99, 013419.

(260) List, N. H.; Saue, T.; Norman, P. Rotationally averaged linear absorption spectra beyond the electric-dipole approximation. Mol. Phys. 2016, 115, 63-74.

(261) Hahn, J. E.; Scott, R. A.; Hodgson, K. O.; Doniach, S.; Desjardins, S. R.; Solomon, E. I. Observation of an electric quadrupole transition in the X-ray absorption spectrum of a $\mathrm{Cu}(\mathrm{II})$ complex. Chem. Phys. Lett. 1982, 88, 595-598.

(262) Josefsson, I.; Kunnus, K.; Schreck, S.; Föhlisch, A.; de Groot, F.; Wernet, P.; Odelius, M. Ab Initio Calculations of X-ray Spectra: Atomic Multiplet and Molecular Orbital Effects in a Multiconfigurational SCF Approach to the L-Edge Spectra of Transition Metal Complexes. J. Phys. Chem. Lett. 2017, 3, 3565-3570. 
(263) Bokarev, S. I.; Dantz, M.; Suljoti, E.; Kühn, O.; Aziz, E. F. State-Dependent Electron Delocalization Dynamics at the Solute-Solvent Interface: Soft-X-Ray Absorption Spectroscopy and Ab Initio Calculations. Phys. Rev. Lett. 2013, 111, 083002.

(264) Pinjari, R. V.; Delcey, M. G.; Guo, M.; Odelius, M.; Lundberg, M. Restricted active space calculations of L-edge X-ray absorption spectra: From molecular orbitals to multiplet states. J. Chem. Phys. 2014, 141, 124116.

(265) Sergentu, D.-C.; Duignan, T. J.; Autschbach, J. Ab Initio Study of Covalency in the Ground versus Core-Excited States and X-ray Absorption Spectra of Actinide Complexes. J. Phys. Chem. Lett. 2018, 9, 5583-5591.

(266) Pinjari, R. V.; Delcey, M. G.; Guo, M.; Odelius, M.; Lundberg, M. Cost and sensitivity of restricted active-space calculations of metal L-edge X-ray absorption spectra. J. Comput. Chem. 2016, 37, 477-486.

(267) Nyvang, A.; Malmqvist, P. A.; Veryazov, V. A generally-contracted Gaussian basis set for photochemistry and photophysics in the range 0-2 keV. 2017; http: //www.molcas.org/VV/poster2_Gothenburg.pdf, Poster at Swedish Theoretical Chemistry meeting 2017.

(268) Cederbaum, L. S.; Domcke, W.; Schirmer, J. Many-body theory of core holes. Phys. Rev. A 1980, 22, 206-222.

(269) Delcey, M. G.; Sørensen, L. K.; Vacher, M.; Couto, R. C.; Lundberg, M. Efficient calculations of a large number of highly excited states for multiconfigurational wavefunctions. J. Comput. Chem. 2019, 40, 1789-1799.

(270) Per-Åke Malmqvist, B. O. R. The CASSCF state interaction method. Chem. Phys. Lett. 1989, 155, 189-194.

(271) Olsen, J.; Jørgensen, P.; Simons, J. Passing the one-billion limit in full configuration-interaction (FCI) calculations. Chem. Phys. Lett. 1990, 169, 463472. 
(272) Grell, G.; Bokarev, S. I.; Winter, B.; Seidel, R.; Aziz, E. F.; Aziz, S. G.; Kühn, O. Multi-reference approach to the calculation of photoelectron spectra including spin-orbit coupling. J. Chem. Phys. 2015, 143, 074104.

(273) Grell, G.; Bokarev, S. I.; Winter, B.; Seidel, R.; Aziz, E. F.; Aziz, S. G.; Kühn, O. Erratum: "Multi-reference approach to the calculation of photoelectron spectra including spin-orbit coupling" [J. Chem. Phys. 143, 074104 (2015)]. J. Chem. Phys. 2016, 145, 089901.

(274) Malmqvist, P. A. Calculation of transition density matrices by nonunitary orbital transformations. Int. J. Quantum Chem. 1986, 30, 479-494.

(275) Cremaschi, P. A theoretical analysis of the four-photon ionization spectrum of the nitric oxide molecule. J. Chem. Phys. 1981, 75, 3944-3953.

(276) Moguilevski, A.; Wilke, M.; Grell, G.; Bokarev, S. I.; Aziz, S. G.; Engel, N.; Raheem, A. A.; Kühn, O.; Kiyan, I. Y.; Aziz, E. F. Ultrafast Spin Crossover in $\left[\mathrm{Fe}^{\mathrm{II}}(\mathrm{bpy})_{3}\right]^{2+}$ : Revealing Two Competing Mechanisms by Extreme Ultraviolet Photoemission Spectroscopy. ChemPhysChem 2017, 18, 465-469.

(277) Golnak, R.; Bokarev, S. I.; Seidel, R.; Xiao, J.; Grell, G.; Atak, K.; Unger, I.; Thürmer, S.; Aziz, S. G.; Kühn, O.; Winter, B.; Aziz, E. F. Joint Analysis of Radiative and Non-Radiative Electronic Relaxation Upon X-ray Irradiation of Transition Metal Aqueous Solutions. Sci. Rep. 2016, 6, 24659.

(278) Norell, J.; Grell, G.; Kühn, O.; Odelius, M.; Bokarev, S. I. Photoelectron shake-ups as a probe of molecular symmetry: $4 \mathrm{~d}$ XPS analysis of $\mathrm{I}_{3}^{-}$in solution. Phys. Chem. Chem. Phys. 2018, 20, 19916-19921.

(279) Josefsson, I.; Eriksson, S. K.; Ottosson, N.; Öhrwall, G.; Siegbahn, H.; Hagfeldt, A.; Rensmo, H.; Björneholm, O.; Odelius, M. Collective hydrogen-bond dynamics dictates the electronic structure of aqueous $\mathrm{I}_{3}^{-}$. Phys. Chem. Chem. Phys. 2013, 15, 20189. 
(280) Piepho, S. B.; Schatz, P. N. Group theory in spectroscopy; John Wiley \& Sons: New York, 1983.

(281) Neese, F.; Solomon, E. I. MCD C-Term Signs, Saturation Behavior, and Determination of Band Polarizations in Randomly Oriented Systems with Spin $S \geq 1 / 2$. Applications to $S=1 / 2$ and $S=5 / 2$. Inorg. Chem. 1999, 38, 1847-1865.

(282) Buckingham, A. D.; Stephens, P. J. Magnetic Optical Activity. Annu. Rev. Phys. Chem. 1966, 17, 399-432.

(283) Stephens, P. J. Theory of Magnetic Circular Dichroism. J. Chem. Phys. 1970, 52, 3489.

(284) Gendron, F.; Fleischauer, V. E.; Duignan, T. J.; Scott, B. L.; Löble, M. W.; Cary, S. K.; Kozimor, S. A.; Bolvin, H.; Neidig, M. L.; Autschbach, J. Magnetic circular dichroism of $\mathrm{UCl}_{6}^{-}$in the ligand-to-metal charge-transfer spectral region. Phys. Chem. Chem. Phys. 2017, 19, 17300-17313.

(285) Bolvin, H. Theoretical Determination of the Excited States and of $g$-Factors of the Creutz-Taube Ion, $\left[\left(\mathrm{NH}_{3}\right)_{5}-\mathrm{Ru}-\text { pyrazine- } \mathrm{Ru}-\left(\mathrm{NH}_{3}\right)_{5}\right]^{5+}$. Inorg. Chem. 2007, 46, $417-427$.

(286) MCD-molcas, tools for calculating MCD spectra with OpenMolcas. https:// github.com/jautschbach/mcd-molcas.git.

(287) Heit, Y. N.; Sergentu, D.-C.; Autschbach, J. Magnetic circular dichroism spectra of transition metal complexes calculated from restricted active space wavefunctions. Phys. Chem. Chem. Phys. 2019, 21, 5586-5597.

(288) Gnuplot 4.6: an interactive plotting program. http://gnuplot.sourceforge.net

(289) Lines, M. E. Orbital Angular Momentum in the Theory of Paramagnetic Clusters. J. Chem. Phys. 1971, 55, 2977-2984.

(290) Chibotaru, L. F.; Iwahara, N. Ising exchange interaction in lanthanides and actinides. New J. Phys. 2015, 17, 103028. 
(291) Noodleman, L.; Case, D. A.; Aizman, A. Broken symmetry analysis of spin coupling in iron-sulfur clusters. J. Am. Chem. Soc. 1988, 110, 1001-1005.

(292) Shoji, M.; Koizumi, K.; Kitagawa, Y.; Kawakami, T.; Yamanaka, S.; Okumura, M.; Yamaguchi, K. A general algorithm for calculation of Heisenberg exchange integrals $J$ in multispin systems. Chem. Phys. Lett. 2006, 432, 343-347.

(293) Langley, S. K.; Wielechowski, D. P.; Vieru, V.; Chilton, N. F.; Moubaraki, B.; Abrahams, B. F.; Chibotaru, L. F.; Murray, K. S. A $\left\{\mathrm{Cr}_{2}^{\mathrm{III}} \mathrm{Dy}_{2}{ }_{2}\right\}$ Single-Molecule Magnet: Enhancing the Blocking Temperature through 3d Magnetic Exchange. Angew. Chem., Int. Ed. 2013, 52, 12014-12019.

(294) Bencini, A.; Gatteschi, D. Electron Paramagnetic Resonance of Exchange Coupled Systems; Springer Berlin Heidelberg, 1990.

(295) Chibotaru, L. F. Theoretical Understanding of Anisotropy in Molecular Nanomagnets. In Molecular Nanomagnets and Related Phenomena; Springer Berlin Heidelberg, 2014; pp 185-229.

(296) Chibotaru, L. F.; Ungur, L. Ab initio calculation of anisotropic magnetic properties of complexes. I. Unique definition of pseudospin Hamiltonians and their derivation. J. Chem. Phys. 2012, 137, 064112.

(297) Chibotaru, L. F. In Ab Initio Methodology for Pseudospin Hamiltonians of Anisotropic Magnetic Complexes; Rice, S. A., Dinner, A. R., Eds.; Advances in Chemical Physics; John Wiley \& Sons, Inc., 2013; Vol. 153; pp 397-519.

(298) Varshalovich, D. A.; Moskalev, A. N.; Khersonskii, V. K. Quantum Theory of Angular Momentum; World Scientific, 1988.

(299) Rudowicz, C. Transformation relations for the conventional $O_{k}^{q}$ and normalised $O_{k}^{\prime q}$ Stevens operator equivalents with $k=1$ to 6 and $-k \leq q \leq k$. J. Phys. C: Solid State Phys. 1985, 18, 1415-1430. 
(300) Rudowicz, C.; Chung, C. Y. The generalization of the extended Stevens operators to higher ranks and spins, and a systematic review of the tables of the tensor operators and their matrix elements. J. Phys.: Condens. Matter 2004, 16, 58255847.

(301) Stoll, S.; Schweiger, A. EasySpin, a comprehensive software package for spectral simulation and analysis in EPR. J. Magn. Reson. 2006, 178, 42-55.

(302) Chibotaru, L. F.; Ungur, L.; Soncini, A. The Origin of Nonmagnetic Kramers Doublets in the Ground State of Dysprosium Triangles: Evidence for a Toroidal Magnetic Moment. Angew. Chem., Int. Ed. 2008, 47, 4126-4129.

(303) Chibotaru, L. F.; Ungur, L.; Aronica, C.; Elmoll, H.; Pilet, G.; Luneau, D. Structure, Magnetism, and Theoretical Study of a Mixed-Valence $\mathrm{Co}_{3}{ }_{3} \mathrm{Co}^{\mathrm{III}}{ }_{4}$ Heptanuclear Wheel: Lack of SMM Behavior despite Negative Magnetic Anisotropy. J. Am. Chem. Soc. 2008, 130, 12445-12455.

(304) Vieru, V.; Iwahara, N.; Ungur, L.; Chibotaru, L. F. Giant exchange interaction in mixed lanthanides. Sci. Rep. 2016, 6 .

(305) Ungur, L.; Thewissen, M.; Costes, J.-P.; Wernsdorfer, W.; Chibotaru, L. F. Interplay of Strongly Anisotropic Metal Ions in Magnetic Blocking of Complexes. Inorg. Chem. 2013, 52, 6328-6337.

(306) Dattani, N. S.; Li Manni, G.; Feller., D.; Koput, J. Submitted.

(307) Dattani, N. Computer Spectrometers. Proceedings of the 72nd International Symposium on Molecular Spectroscopy. 2017.

(308) Gagliardi, L.; Lindh, R.; Karlström, G. Local properties of quantum chemical systems: The LoProp approach. J. Chem. Phys. 2004, 121, 4494-4500.

(309) Schaftenaar, G.; Noordik, J. H. Molden: A pre- and post-processing program for molecular and electronic structures. J. Comput.-Aided Mol. Des. 2000, 14, 123134. 
(310) Kovačević, G.; Veryazov, V. LUSCUS: molecular viewer and editor for MOLCAS. J. Cheminf. 2015, \%, 16.

(311) Williams, S. In Free As in Freedom: Richard Stallman's Crusade for Free Software, 1st ed.; Petrycki, L., Ed.; O’Reilly \& Associates, Inc.: Sebastopol, CA, USA, 2002.

(312) SAGIT, a grid generator distributed under the LGPL license. https:// sourceforge.net/projects/sagit/.

(313) Pegamoid, an orbital viewer especially suited for use with OpenMolcas. https: //pypi.org/project/Pegamoid.

(314) Plasser, F.; Wormit, M.; Dreuw, A. New tools for the systematic analysis and visualization of electronic excitations. I. Formalism. J. Chem. Phys. 2014, 141, 024106.

(315) Dong, S. S.; Gagliardi, L.; Truhlar, D. G. Nature of the $1^{1} B_{u}$ and $2^{1} A_{g}$ Excited States of Butadiene and the Goldilocks Principle of Basis Set Diffuseness. J. Chem. Theory Comput. 2019, 15, 4591-4601.

(316) Amos, A. T.; Hall, G. G. Single Determinant Wave Functions. Proc. R. Soc. London, Ser. A 1961, 263, 483-493.

(317) Martin, R. L. Natural transition orbitals. J. Chem. Phys. 2003, 118, 4775-4777.

(318) Mayer, I. Identifying a pair of interacting chromophores by using SVD transformed CIS wave functions. Chem. Phys. Lett. 2007, 443, 420-425.

(319) Kawata, I.; Nitta, H. An excited state paired interacting orbital method. J. Chem. Phys. 2012, 136, 064109.

(320) Malmqvist, P. A.; Veryazov, V. The binatural orbitals of electronic transitions. Mol. Phys. 2012, 110, 2455-2464.

(321) Stewart, G. W. On the Early History of the Singular Value Decomposition. SIAM Rev. 1993, 35, 551-566. 
(322) Stewart, G. W. The decompositional approach to matrix computation. Comput. Sci. Eng. 2000, 2, 50-59.

(323) Plasser, F.; Mewes, S. A.; Dreuw, A.; González, L. Detailed Wave Function Analysis for Multireference Methods: Implementation in the Molcas Program Package and Applications to Tetracene. J. Chem. Theory Comput. 2017, 13, 5343-5353.

(324) Plasser, F.; Thomitzni, B.; Bäppler, S. A.; Wenzel, J.; Rehn, D. R.; Wormit, M.; Dreuw, A. Statistical analysis of electronic excitation processes: Spatial location, x compactness, charge transfer, and electron-hole correlation. J. Comput. Chem. 2015, 36, 1609-1620.

(325) Bäppler, S. A.; Plasser, F.; Wormit, M.; Dreuw, A. Exciton analysis of many-body wave functions: Bridging the gap between the quasiparticle and molecular orbital pictures. Phys. Rev. A 2014, 90, 052521.

(326) Plasser, F.; Lischka, H. Analysis of Excitonic and Charge Transfer Interactions from Quantum Chemical Calculations. J. Chem. Theory Comput. 2012, 8, 2777-2789.

(327) Mewes, S. A.; Mewes, J.-M.; Dreuw, A.; Plasser, F. Excitons in poly(para phenylene vinylene): a quantum-chemical perspective based on high-level ab initio calculations. Phys. Chem. Chem. Phys. 2016, 18, 2548-2563.

(328) Plasser, F.; Bäppler, S. A.; Wormit, M.; Dreuw, A. New tools for the systematic analysis and visualization of electronic excitations. II. Applications. J. Chem. Phys. 2014, 141, 024107.

(329) Plasser, F.; Dreuw, A. High-Level Ab Initio Computations of the Absorption Spectra of Organic Iridium Complexes. J. Phys. Chem. A 2015, 119, 1023-1036.

(330) Head-Gordon, M. Characterizing unpaired electrons from the one-particle density matrix. Chem. Phys. Lett. 2003, 372, 508-511.

(331) Plasser, F.; Pašalić, H.; Gerzabek, M. H.; Libisch, F.; Reiter, R.; Burgdörfer, J.; 
Müller, T.; Shepard, R.; Lischka, H. The Multiradical Character of One- and TwoDimensional Graphene Nanoribbons. Angew. Chem., Int. Ed. 2013, 52, 2581-2584.

(332) Nogueira, J. J.; Plasser, F.; González, L. Electronic delocalization, charge transfer and hypochromism in the UV absorption spectrum of polyadenine unravelled by multiscale computations and quantitative wavefunction analysis. Chem. Sci. 2017, $8,5682-5691$.

(333) Kautny, P.; Glöcklhofer, F.; Kader, T.; Mewes, J.-M.; Stöger, B.; Fröhlich, J.; Lumpi, D.; Plasser, F. Charge-transfer states in triazole linked donor-acceptor materials: strong effects of chemical modification and solvation. Phys. Chem. Chem. Phys. 2017, 19, 18055-18067.

(334) Mewes, S. A.; Plasser, F.; Krylov, A.; Dreuw, A. Benchmarking Excited-State Calculations Using Exciton Properties. J. Chem. Theory Comput. 2018, 14, 710725.

(335) Mai, S.; Plasser, F.; Dorn, J.; Fumanal, M.; Daniel, C.; González, L. Quantitative wave function analysis for excited states of transition metal complexes. Coord. Chem. Rev. 2018, 361, 74-97.

(336) Mewes, S. A.; Plasser, F.; Dreuw, A. Universal Exciton Size in Organic Polymers is Determined by Nonlocal Orbital Exchange in Time-Dependent Density Functional Theory. J. Phys. Chem. Lett. 2017, 8, 1205-1210.

(337) Head-Gordon, M.; Grana, A. M.; Maurice, D.; White, C. A. Analysis of Electronic Transitions as the Difference of Electron Attachment and Detachment Densities. J. Phys. Chem. 1995, 99, 14261-14270.

(338) Wesolowski, T. A.; Warshel, A. Frozen density functional approach for ab initio calculations of solvated molecules. J. Phys. Chem. 1993, 97, 8050-8053.

(339) Wesołowski, T. A. Embedding a multideterminantal wave function in an orbitalfree environment. Phys. Rev. A 2008, 77, 012504. 
(340) Wesolowski, T. A.; Shedge, S.; Zhou, X. Frozen-Density Embedding Strategy for Multilevel Simulations of Electronic Structure. Chem. Rev. (Washington, DC, U. S.) $\mathbf{2 0 1 5}, 115,5891-5928$.

(341) Laktionov, A.; Chemineau-Chalaye, E.; Wesolowski, T. A. Frozen-density embedding theory with average solvent charge densities from explicit atomistic simulations. Phys. Chem. Chem. Phys. 2016, 18, 21069-21078.

(342) Zech, A.; Aquilante, F.; Wesolowski, T. A. Homogeneity properties of the embedding potential in frozen-density embedding theory. Mol. Phys. 2015, 114, 1199 1206.

(343) Zech, A.; Aquilante, F.; Wesolowski, T. A. Orthogonality of embedded wave functions for different states in frozen-density embedding theory. J. Chem. Phys. 2015, $143,164106$.

(344) Aquilante, F.; Wesołowski, T. A. Self-consistency in frozen-density embedding theory based calculations. J. Chem. Phys. 2011, 135, 084120.

(345) Prager, S.; Zech, A.; Aquilante, F.; Dreuw, A.; Wesolowski, T. A. First time combination of frozen density embedding theory with the algebraic diagrammatic construction scheme for the polarization propagator of second order. J. Chem. Phys. 2016, 144, 204103.

(346) Humbert-Droz, M.; Zhou, X.; Shedge, S. V.; Wesolowski, T. A. How to choose the frozen density in Frozen-Density Embedding Theory-based numerical simulations of local excitations? Theor. Chem. Acc. 2014, 133, 1405.

(347) Almlöf, J.; Taylor, P. R. General contraction of Gaussian basis sets. I. Atomic natural orbitals for first- and second-row atoms. J. Chem. Phys. 1987, 86, 40704077.

(348) Dunning, T. H. Gaussian basis sets for use in correlated molecular calculations. I. The atoms boron through neon and hydrogen. J. Chem. Phys. 1989, 90, 1007-1023. 
(349) Jensen, F. Polarization consistent basis sets: Principles. J. Chem. Phys. 2001, 115, 9113-9125.

(350) Widmark, P.-O; Malmqvist, P.-A.; Roos, B. O. Density matrix averaged atomic natural orbital (ANO) basis sets for correlated molecular wave functions. Theor. Chim. Acta 1990, 77, 291-306.

(351) Pierloot, K.; Dumez, B.; Widmark, P.-O.; Roos, B. O. Density matrix averaged atomic natural orbital (ANO) basis sets for correlated molecular wave functions. Theor. Chim. Acta 1995, 90, 87-114.

(352) Roos, B. O.; Lindh, R.; Malmqvist, P.-A.; Veryazov, V.; Widmark, P.-O. Main Group Atoms and Dimers Studied with a New Relativistic ANO Basis Set. J. Phys. Chem. A 2004, 108, 2851-2858.

(353) Widmark, P.-O.; Zobel, J. P.; Vysotskiy, V. P.; Tsuchiya, T.; Veryazov, V. New compact density matrix averaged ANO basis sets for relativistic calculations. J. Chem. Phys. 2018, 149, 194102.

(354) Johansson, M.; Veryazov, V. Automatic procedure for generating symmetry adapted wavefunctions. J. Cheminf. 2017, 9, 8.

(355) msym, a library for symmetrization distributed under the MIT license. https: //gitlab.com/mcodev31/libmsym.

(356) Ninomiya, K.; Nagatomo, T.; Kubo, K.; Ito, T. U.; Higemoto, W.; Kita, M.; Shinohara, A.; Strasser, P.; Kawamura, N.; Shimomura, K.; Miyake, Y.; Saito, T. Development of Nondestructive and Quantitative Elemental Analysis Method Using Calibration Curve between Muonic X-ray Intensity and Elemental Composition in Bronze. Bull. Chem. Soc. Jpn. 2012, 85, 228-230.

(357) Terada, K.; Ninomiya, K.; Osawa, T.; Tachibana, S.; Miyake, Y.; Kubo, M. K.; Kawamura, N.; Higemoto, W.; Tsuchiyama, A.; Ebihara, M.; Uesugi, M. A new X-ray fluorescence spectroscopy for extraterrestrial materials using a muon beam. Sci. Rep. 2014, 4 . 
(358) Ninomiya, K.; Kubo, M. K.; Nagatomo, T.; Higemoto, W.; Ito, T. U.; Kawamura, N.; Strasser, P.; Shimomura, K.; Miyake, Y.; Suzuki, T.; Kobayashi, Y.; Sakamoto, S.; Shinohara, A.; Saito, T. Nondestructive Elemental Depth-Profiling Analysis by Muonic X-ray Measurement. Anal. Chem. 2015, 87, 4597-4600.

(359) Ugandi, M.; Fdez. Galván, I.; Widmark, P.-O.; Lindh, R. Uncontracted basis sets for ab initio calculations of muonic atoms and molecules. Int. J. Quantum Chem. 2018, 118, e25755.

(360) Mohallem, J. R.; Diniz, L. G.; Dutra, A. S. Separation of motions of atomic cores and valence electrons in molecules. Chem. Phys. Lett. 2011, 501, 575-579. 
Graphical TOC Entry

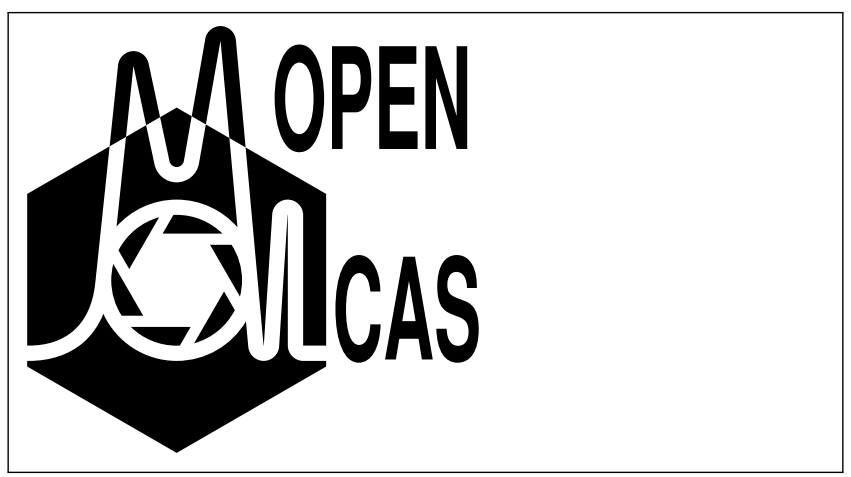




\section{Supporting information for:}

\section{OpenMolcas: From source code to insight}

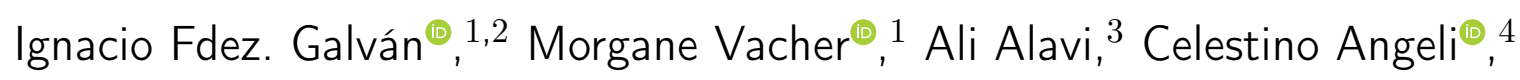

Francesco Aquilante ${ }^{\oplus}, 5$ Jochen Autschbach ${ }^{\oplus}, 6$ Jie J. Bac ${ }^{\oplus, 7}$ Sergey I.

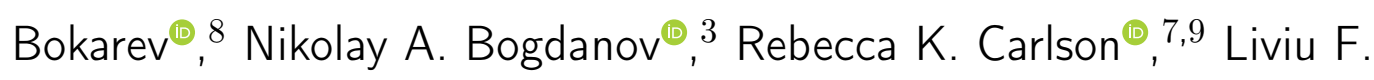

Chibotaru, ${ }^{10}$ Joel Creutzberg, ${ }^{11,12}$ Nike Dattani, ${ }^{13}$ Mickaël G. Delcey ${ }^{0}, 1$ Sijia S.

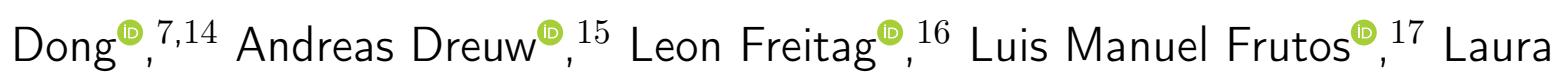

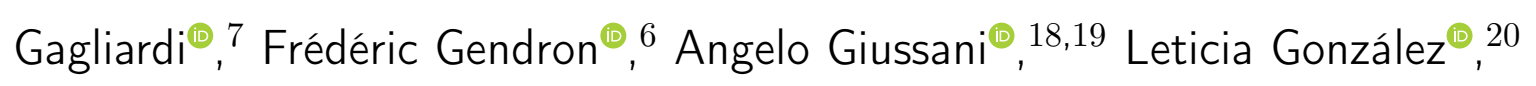

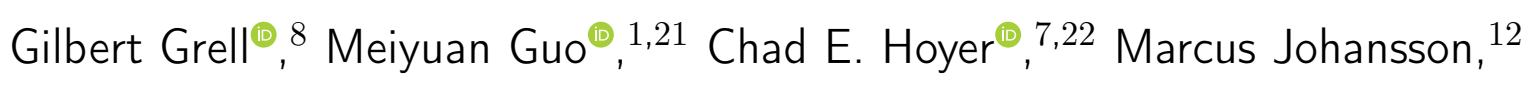

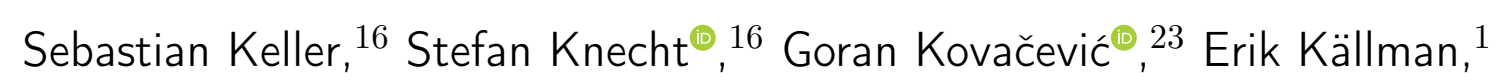

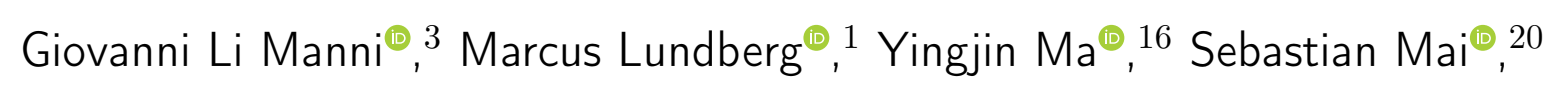
João Pedro Malhado, ${ }^{24}$ Per Åke Malmqvist॰ ${ }^{12}$ Philipp Marquetandø 20

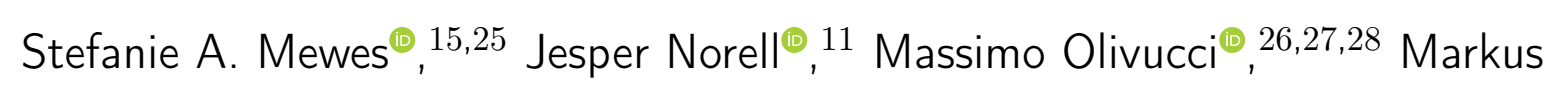

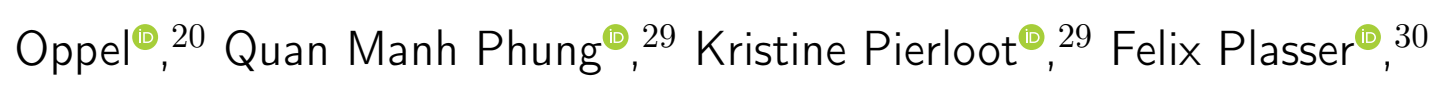

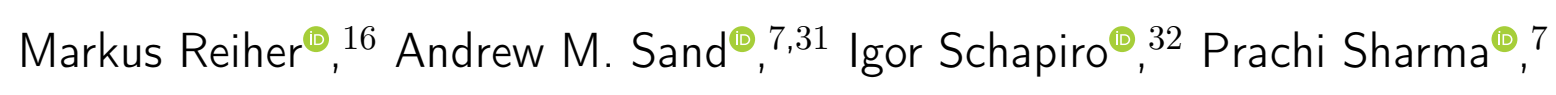

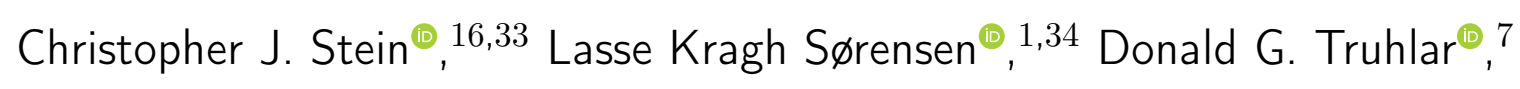

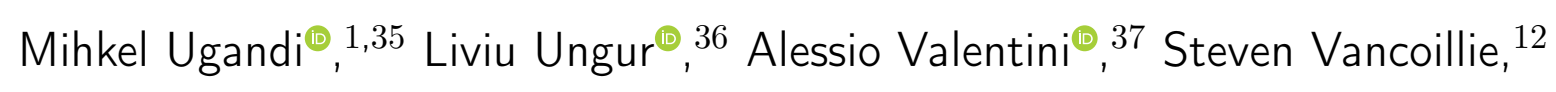

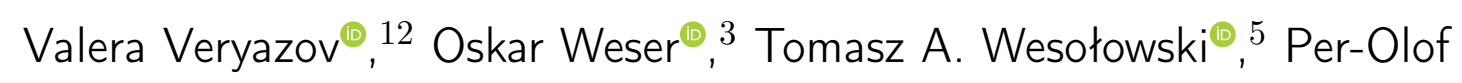
Widmark, ${ }^{12}$ Sebastian Wouters, ${ }^{38}$ Alexander Zechø, ${ }^{5}$ J. Patrick Zobel, 12 and 
1Department of Chemistry - Angström Laboratory, Uppsala University, P.O. Box 538, SE-75121 Uppsala, Sweden

2Department of Chemistry - BMC, Uppsala University, P.O. Box 576, SE-751 23 Uppsala, Sweden

3Max Planck Institut für Festkörperforschung, Heisenbergstraße 1, 70569 Stuttgart, Germany

4Dipartimento di Scienze Chimiche e Farmaceutiche, Università di Ferrara, Via Luigi Borsari 46, 44121 Ferrara, Italy

5Département de Chimie Physique, Université de Genève, 30 quai Ernest-Ansermet, CH-1211 Genève 4, Switzerland 6Department of Chemistry, University at Buffalo, State University of New York. Buffalo, NY 14260-3000, USA

7 Department of Chemistry, Chemical Theory Center, and Minnesota Supercomputing Institute, University of Minnesota, Minneapolis, MN 55455-0431, USA

8Institut für Physik, Universität Rostock, Albert-Einstein-Str. 23-24, 18059 Rostock, Germany

9Present address: Theoretical Division, Los Alamos National Laboratory, Los Alamos, $N M 87545, U S A$

10 Theory of Nanomaterials Group, University of Leuven, Celestijnenlaan 200F, Leuven 3001, Belgium

11Department of Physics, AlbaNova University Center, Stockholm University, SE-106 91 Stockholm, Sweden

12Division of Theoretical Chemistry, Kemicentrum, Lund University, P. O. Box 124, SE-221 00 Lund, Sweden

13Harvard Smithsonian Center for Astrophysics, Cambridge, Massachusetts, USA 14Present address: Materials Science Division and Center for Molecular Engineering, Argonne National Laboratory, Lemont, IL 60439, USA

15Interdisciplinary Center for Scientific Computing, Heidelberg University, Im 
Neuenheimer Feld 205 A, 69120 Heidelberg, Germany

16Laboratorium für Physikalische Chemie, ETH Zürich, Vladimir-Prelog-Weg 2, 8093

Zürich, Switzerland

17Departamento de Química Analítica, Química Física e Ingeniería Química, and

Instituto de Investigación Química "Andrés M. del Río". Universidad de Alcalá.

E-28871 Alcalá de Henares, Madrid, Spain

18Department of Chemistry, University College London, 20 Gordon Street, London

WC1H 0AJ, United Kingdom

19Instituto de Ciencia Molecular, Universitat de València, Apartado 22085, ES-46071

Valencia, Spain

20Institute of Theoretical Chemistry, Faculty of Chemistry, University of Vienna,

Währinger Straße 17, 1090 Vienna, Austria

21Present address: Division of Chemical Physics, Chemical Center, Lund University,

SE-221 00 Lund, Sweden

22Present address: Department of Chemistry, University of Washington, Seattle, WA 98195, USA

23Division of Materials Physics, Ruđer Bošković Institute, P.O.B. 180, Bijenička 54, HR-10002 Zagreb, Croatia

24Department of Chemistry, Imperial College London, London SW7 2AZ, United Kingdom

25Centre for Theoretical Chemistry and Physics, The New Zealand Institute for

Advanced Study (NZIAS), Massey University Albany, Private Bag 102904, Auckland 0632, New Zealand

26Department of Biotechnology, Chemistry and Pharmacy, University of Siena, via A.

Moro 2, 53100 Siena, Italy

27 Department of Chemistry, Bowling Green State University, Bowling Green, OH

$$
\text { 43403, USA }
$$

28 USIAS and Institut de Physique et Chimie des Matériaux de Strasbourg, Université de Strasbourg-CNRS 67034 Strasbourg, France 
29Department of Chemistry, KU Leuven, Celestijnenlaan 200F, Leuven 3001, Belgium 30Department of Chemistry, Loughborough University, Loughborough, LE11 3TU, United Kingdom

31Present address: Department of Chemistry, Butler University, 4600 Sunset Avenue, Indianapolis, IN 46208, USA

32Institute of Chemistry, The Hebrew University of Jerusalem, Jerusalem, Israel 33Present address: Chemical Sciences Division, Lawrence Berkeley National Laboratory, 1 Cyclotron Road, Berkeley CA 94720, USA

34Present address: Theoretical Chemistry and Biology, School of Engineering Sciences in Chemistry, Biotechnology and Health (CBH), Royal Institute of Technology (KTH),

SE-106 91 Stockholm, Sweden

35Present address: Lehrstuhl für Theoretische Chemie, Ruhr-Universität Bochum,

$$
\text { D-44780 Bochum, Germany }
$$

36 Department of Chemistry, National University of Singapore

37 Theoretical Physical Chemistry, Research Unit MolSys, Allée du 6 Août, 11, 4000

Liège, Belgium

38Brantsandpatents, Pauline van Pottelsberghelaan 24, 9051 Sint-Denijs-Westrem, Belgium

39 Uppsala Center for Computational Chemistry (UC $\left.C_{3}\right)$, Uppsala University, P.O. Box 596, SE-75124 Uppsala, Sweden

E-mail: roland.lindh@kemi.uu.se

\section{Contents}

S1 Stochastic-CASSCF in action $\quad$ S5

\begin{tabular}{lll}
\hline S2 & DMRG-CASPT2 input example & S7
\end{tabular}

\begin{tabular}{lll}
\hline S3 & QCMAQUIS input examples & S8
\end{tabular}

S4 Spin state energetics of iron(II) porphyrin with OpenMolcas-
CheMPS2 
S4.1 Computational details of $\mathrm{FeP} \ldots \ldots \ldots \ldots$. . . . . . . . . . . . . . . .

S4.2 Active orbitals of FeP . . . . . . . . . . . . . . . . . . . . . . . . . . . . . . . . . . . . . . . . . . . .

S4.3 Cartesian coordinates of FeP . . . . . . . . . . . . . . . . . . . . . S13

S4.4 Convergence and performance . . . . . . . . . . . . . . . . S15

\begin{tabular}{lll}
\hline S5 & Equilibrium structure of ferrocene & S17
\end{tabular}

S5.1 Computational details . . . . . . . . . . . . . S17

S5.2 Active orbitals of ferrocene . . . . . . . . . . . . . . . . . . . . S18

S5.3 Cartesian coordinates of ferrocene . . . . . . . . . . . . . . . . S18

S6 Computational details for the DMRG-NEVPT2 example S20

S7 Multiconfiguration pair-density functional theory. Input examples S20

S7.1 General input for single-point MC-PDFT calculations . . . . . . . . . S20

S7.2 $\quad$ SS-CAS-PDFT gradient . . . . . . . . . . . . . . . . . S20

S7.3 Scaling exchange and correlation terms in MC-PDFT and KS-DFT . . S21

S7.4 $\quad$ SI-PDFT . . . . . . . . . . . . . . . . . . . . . . . . . . . . S S22

S7.5 General input for a single-point DMRG-PDFT calculation . . . . . . . S23

S8 Conical intersection optimization and characterization $\quad$ S24

\begin{tabular}{lll}
\hline S9 & Setup for a single SHARC trajectory & S25
\end{tabular}

S9.1 $\quad$ Input file . . . . . . . . . . . . . . . . . . . . . . . . . S S25

S9.2 $\quad$ Geometry file $\ldots \ldots \ldots \ldots \ldots$. . . . . . . . . . . . . . . . . . S26

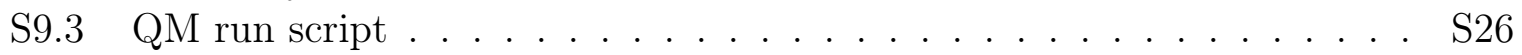

S9.4 Template file. . . . . . . . . . . . . . . . . . . . . . . . . S26

S9.5 Resource file . . . . . . . . . . . . . . . . . . . . . . . . . . S27

S9.6 Running SHARC . . . . . . . . . . . . . . . . . . . . . . . . . . . . . . . . . . . . . . . . . . . . . . . . . . . . . .

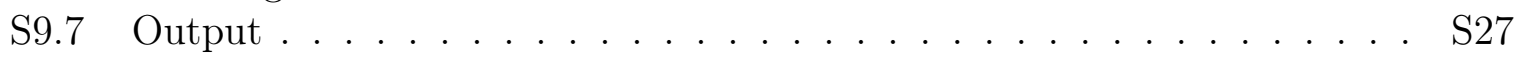

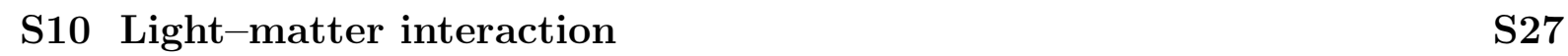

S11 Multi-configurational Dyson orbitals in RASST $\quad$ S30

\begin{tabular}{|l|l|} 
S12 Orbital properties & S31
\end{tabular}

S12.1 Printing orbital second moment $\left\langle r^{2}\right\rangle$ for all orbitals to identify Rydberg

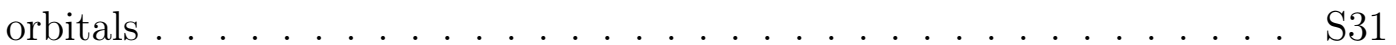

\begin{tabular}{|ll}
\hline S13 Binatural orbitals & S33
\end{tabular}

\begin{tabular}{ll}
\hline S14 The WFA module & S33
\end{tabular}

\begin{tabular}{|ll|}
\hline S15 Example of FDET calculation & S34
\end{tabular}

\begin{tabular}{ll}
\hline References & S37
\end{tabular}

\section{Stochastic-CASSCF in action}

A test case is described here to show how to prepare OpenMolcas and NECI inputs to perform Stochastic-CASSCF calculations. In the OpenMolcas input the NECI keyword 
is necessary (within the RASSCF module) to enable the FCIQMC as CI eigensolver. By default the uncoupled mode is used, which is available in a standard compilation of OpenMolcas (the user is responsible to download and compile NECI program externally). When OpenMolcas is compiled with the embedded NECI (as a git submodule), the input keyword EMBD switches to the embedded mode. The keyword DMPO is used to generate the DUMPFILE related to the chosen active space and input orbitals (the ones linked to the INPORB). The keywords for controlling the NECI run from OpenMolcas are chosen to match as closely as possible the corresponding ones in NECI. The keyword TOTAlwalkers sets the target number of walkers. The use of the DEFInedet keyword is also adviced to set an initial reference determinant for the FCIQMC dynamics. It is an array of integers indicating the occupied alpha and beta spin-orbitals in the MO orbitals as ordered in the INPORB file. If the population on the user specified reference determinant is reduced with respect to some other determinant within a given ratio, the reference determinant is automatically changed by the NECI program. The keyword RDMSamplingiters defines the number of iterations for accumulating RMDs. The keyword CALCrdmonfly takes two integers: the first entry specifies equilibration delay before RDM sampling is started, the second entry is the period for averaging RDM and RDM energy. Other keywords are optional.

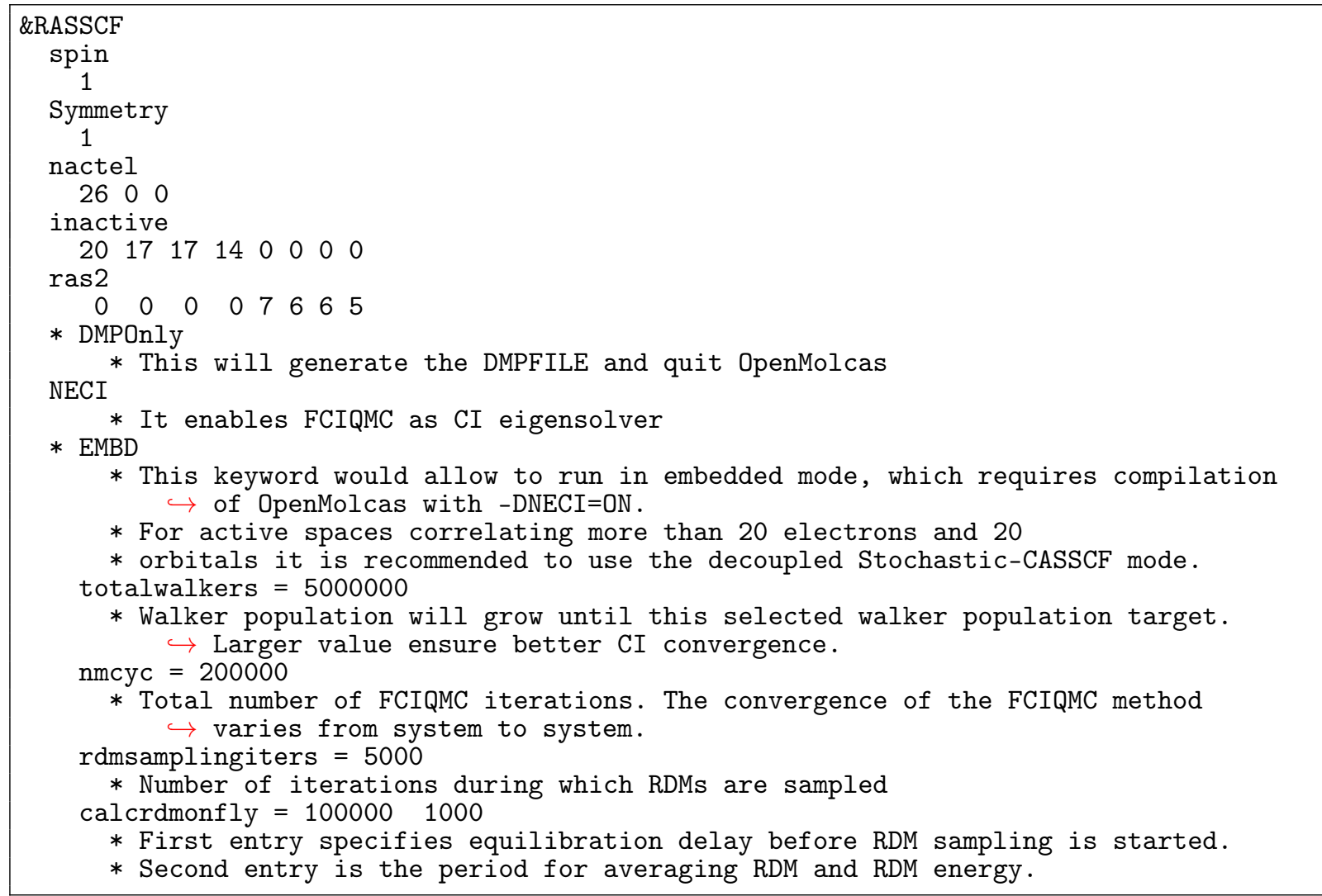

Listing S1: A possible OpenMolcas input for the porphyrin molecule within the $D_{2 \mathrm{~h}}$ point group.

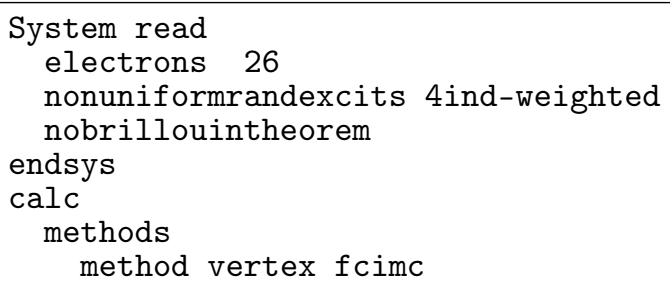




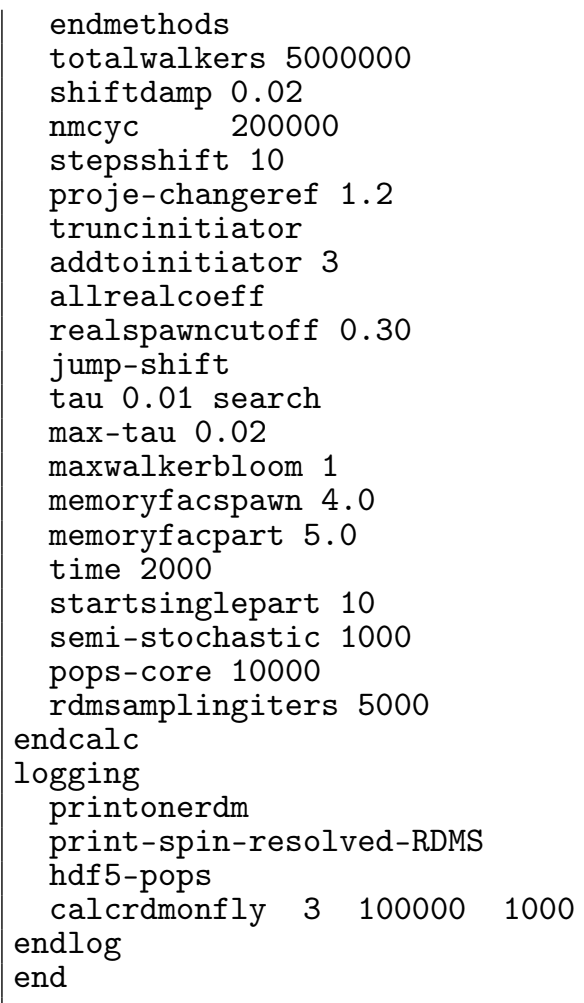

Listing S2: List of relevant input keywords used by the FCIQMC program.

\section{DMRG-CASPT2 input example}

In listing S3, we present an input example to perform a geometry optimization calculation of benzene using DMRG-CASPT2 numerical gradients on a 12-core (24 threads) CPU. In the RASSCF module, the keyword DMRG specifies the maximum number of renormalized states (or virtual bond dimension $m$ ) in each microiteration. With the keyword 3RDM, the three-particle and Fock matrix contracted with the four-particle reduced density matrices (3-RDM and F.4-RDM) are evaluated. In the CASPT2 module, the keyword CHEMps2 activates the DMRG-CASPT2 calculation and OpenMolcas will skip the calculations of the reduced density matrices. Note that only state-specific calculations are supported.

Because the effective number of displacements in this example is 12 , we used MOLCAS_NPROCS=12. The number of CheMPS2 threads can be set using the environment variable OMP_NUM_THREADS.

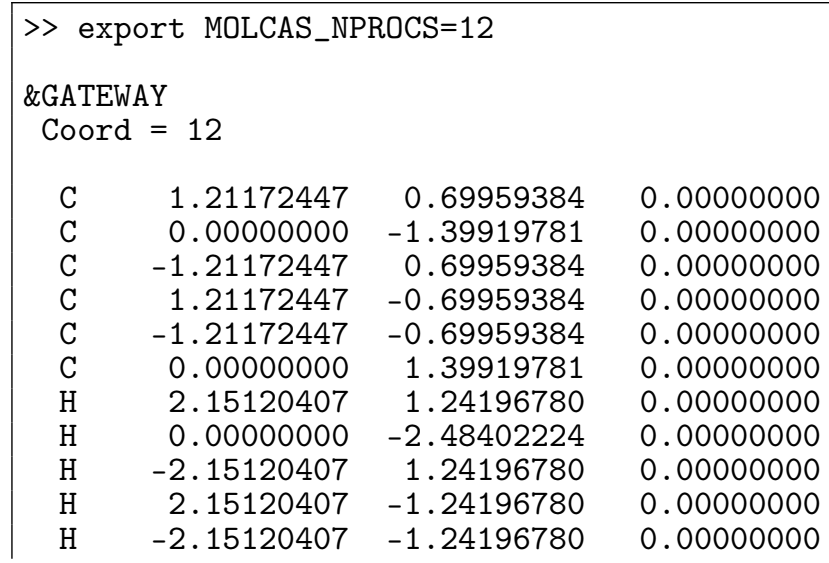




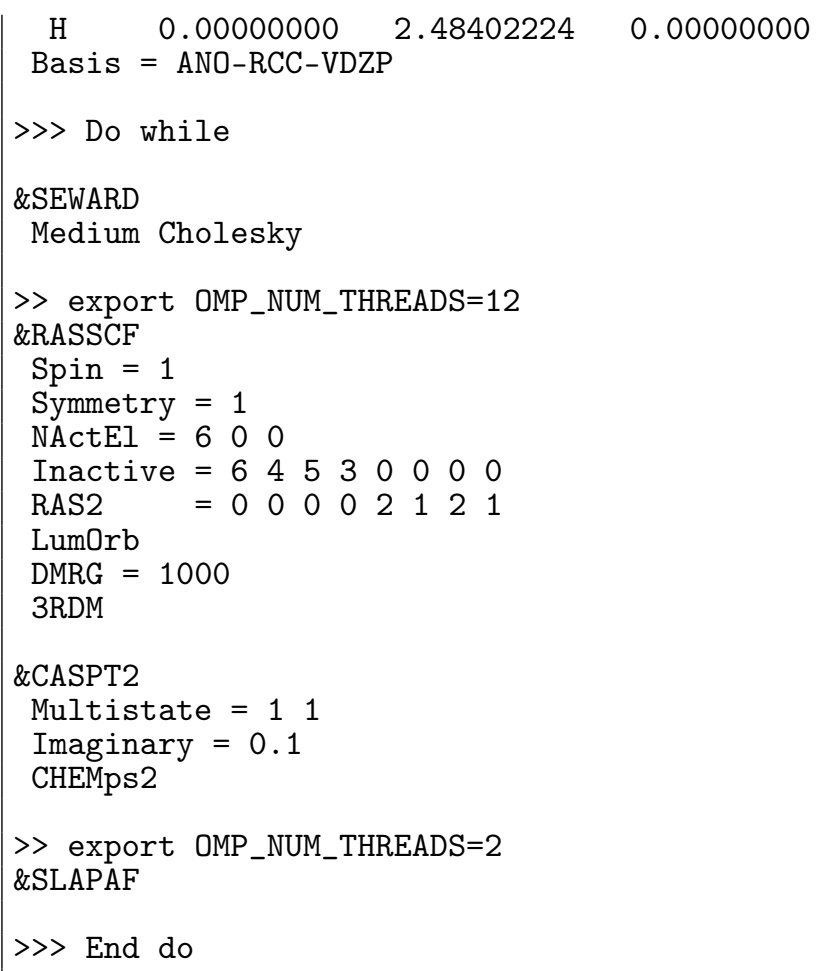

Listing S3: Geometry optimization of benzene using DMRG-CASPT2 numerical gradients.

\section{QCMAQUIS input examples}

Listings S4 to S6 provide input examples for DMRG-SCF, MPSSI and DMRG-NEVPT2 calculations performed with the OpenMOLCAS-QCMAQUIS interface. For more information and a detailled documentation, the reader may consult the QCMaquis interface manual (https://scine.ethz.ch/static/download/qcmaquis_manual.pdf).

In listing S4, the DMRGSCF module is employed to drive the DMRG-SCF optimization with the keyword ActiveSpaceOptimizer=QCMaquis. Its input is similar to the input of the RASSCF module, but contains an additional DMRGSettings... EndDMRGSettings block. Two settings are mandatory to perform a DMRG calculation, namely the maximum bond dimension specified in the max_bond_dimension option and the maximum number of sweeps, denoted nsweeps. Non DMRG-specific options (corresponding to the "old-style" RASSCF input) are specified in the 00ptimizationSettings block and are otherwise identical to the RASSCF input for an analogous CASSCF calculation.

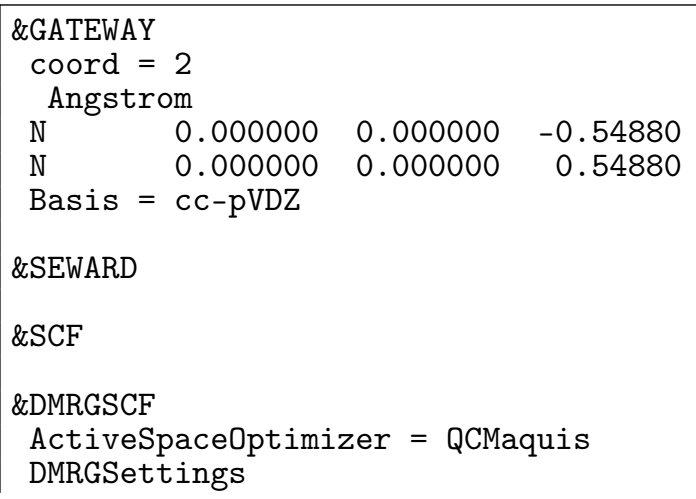




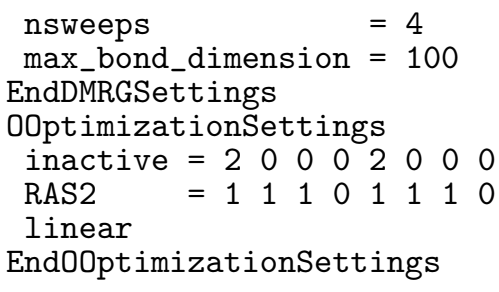

Listing S4: MRG-SCF optimization of the ground state of $\mathrm{N}_{2}$, with a $(6,6)$ active space.

In listing [S5, we show an example of MPSSI input. First, we perform a singlet and a triplet DMRG-SCF optimization with the DMRGSCF module. We save the HDF5 output files of the singlet and triplet calculation to prevent them from being overwritten, and, additionally, save also the QCMaquis MPS, which is stored in a separate folder \$Project.checkpoint_state.0.h5. The information about the QCMaquis checkpoint folder name which is required for the MPSSI calculation is stored in the DMRGSCF HDF5 file \$Project.dmrgscf.h5, but as the QCMaquis checkpoint folder has been renamed, the new name must be stored in the DMRGSCF HDF5 file too. This is accomplished with a call to the qcm_checkpoint_rename.py script. Finally, MPSSI is run, with an input identical to a RASSI input.

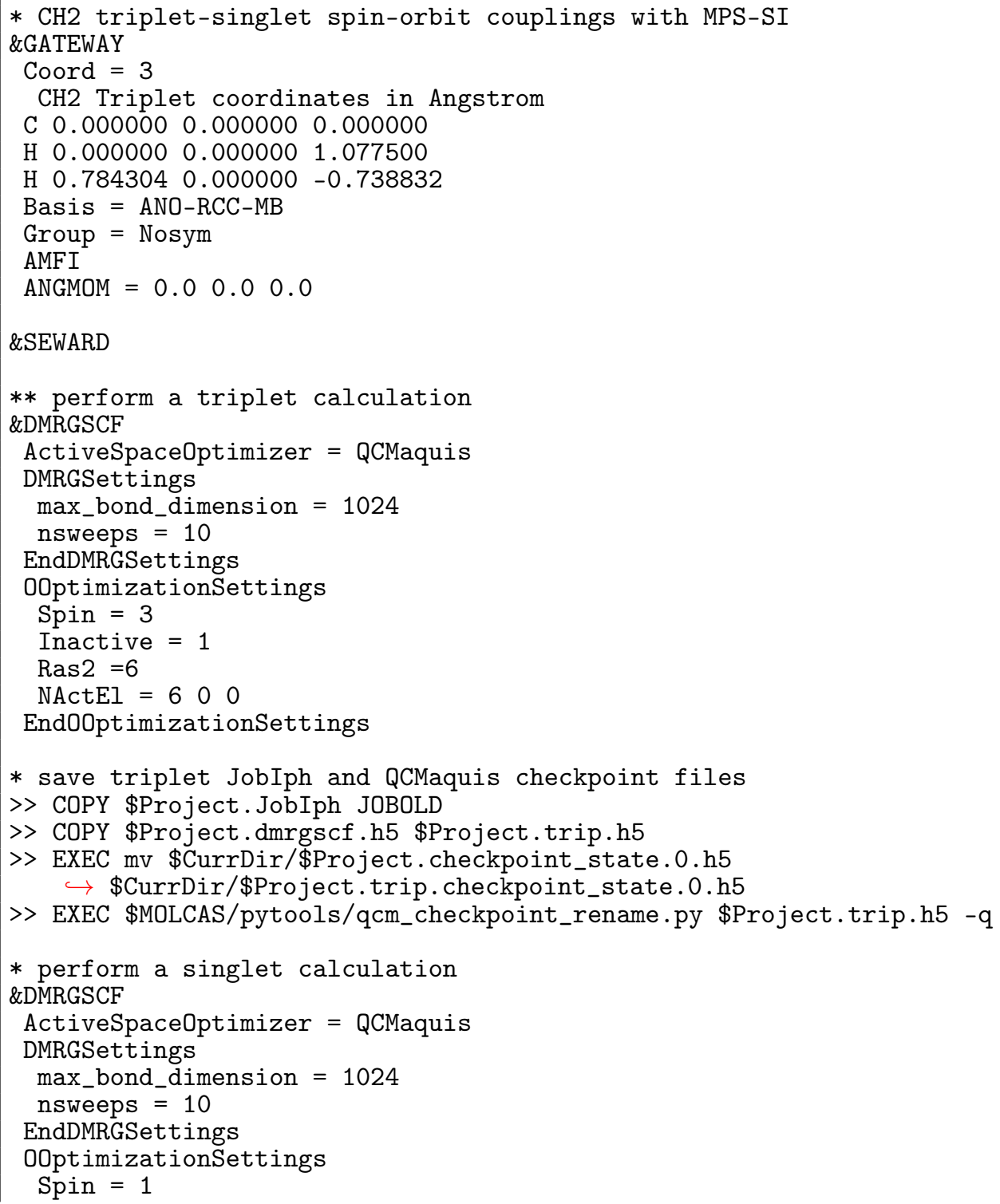




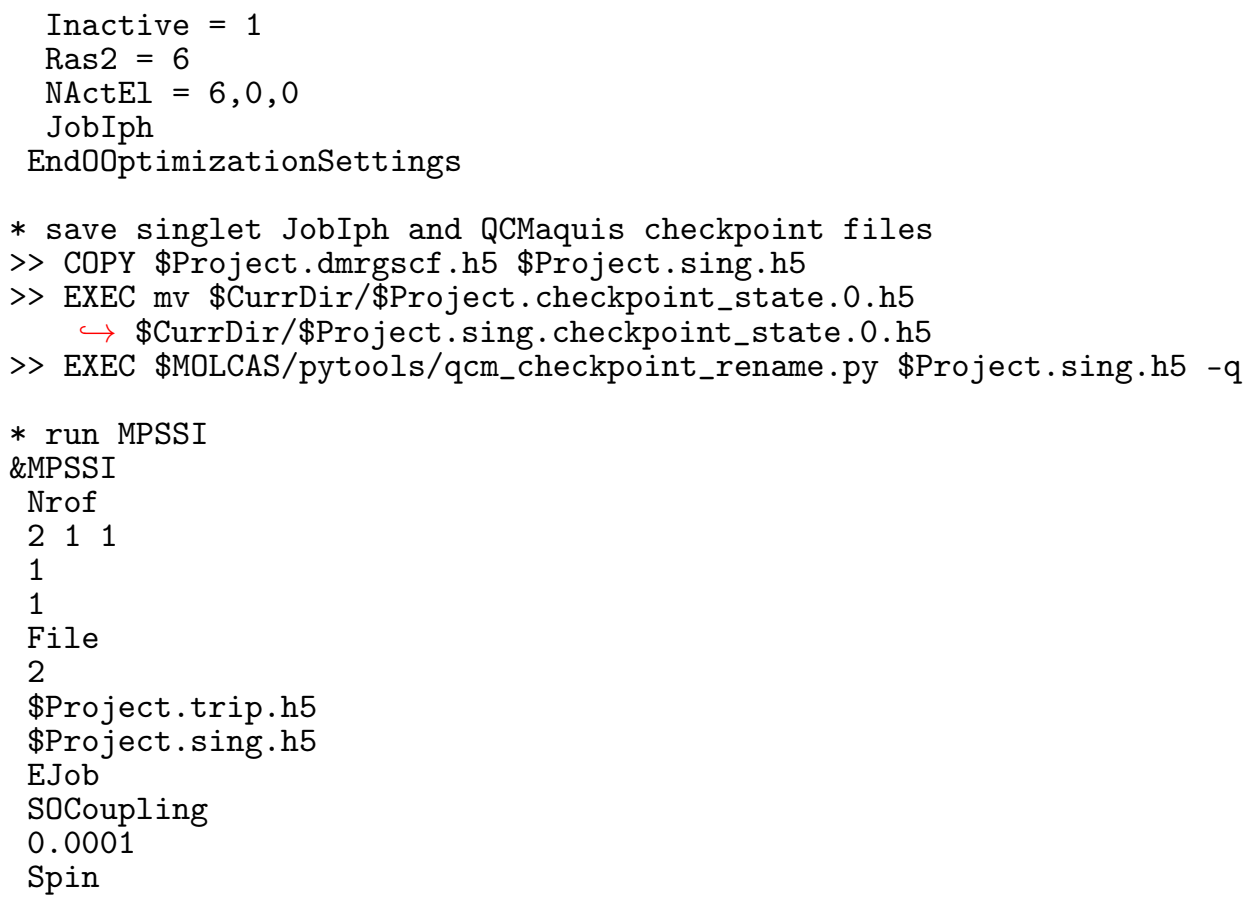

Listing S5: Spin-orbit couplings calculation with MPSSI between the lowest singlet and triplet state of methylene for a $(6,6)$ active space.

Listing S6 shows a DMRG-NEVPT2 example. The DMRGSCF module gains two additional options, NEVPT2Prep, which prepares a NEVPT2 calculation, and EvRDM, which explicitly evaluates the four-particle reduced density matrix (4-RDM). For active spaces larger than 10-11 orbitals, an external distributed parallel 4-RDM evaluation, as described in the QCMaquis interface manual is recommended. Afterwards, a MOTRA call with the HDF5 option is required, and, if Cholesky decomposition of two-electron integrals is employed, also options CTOnly and Kpq. We conclude the input with the call to the NEVPT2 module.

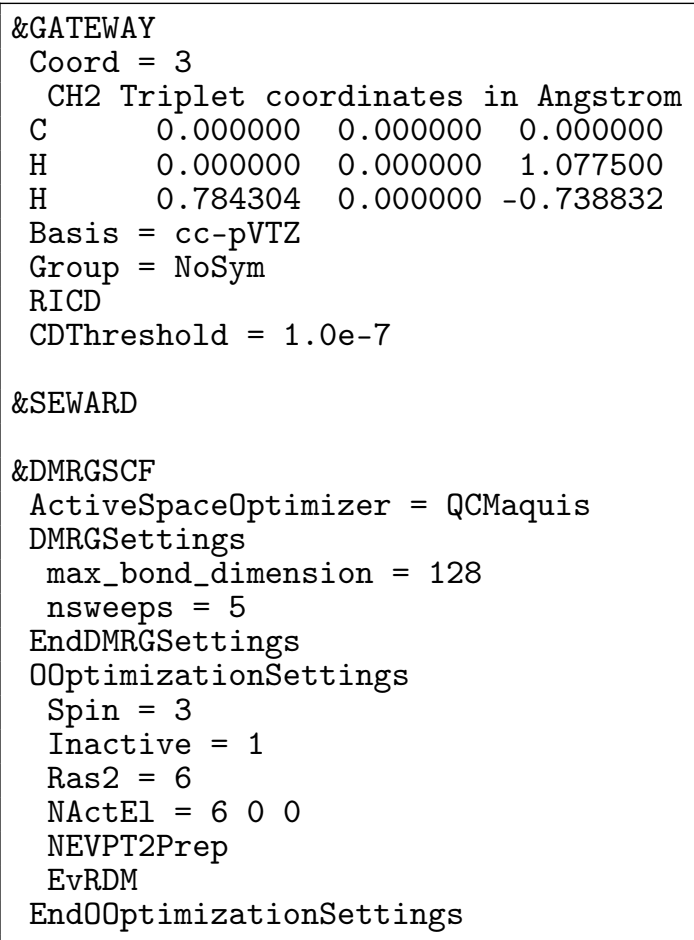




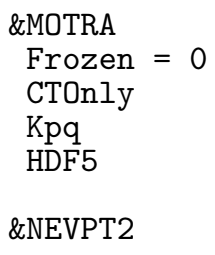

Listing S6: A DMRG-SC-NEVPT2 and DMRG-PC-NEVPT2 calculation of the lowest triplet state in methylene with a $(6,6)$ active space.

\section{Spin state energetics of iron(II) porphyrin with OpenMolcas-CheMPS2}

\section{Computational details of FeP}

- Active space: $\operatorname{CAS}(8,11)$ and $\operatorname{CAS}(26,27)$, see fig. S1.

- Cartesian coordinates from PBE0/def2-TZVP, see tables S2 and S3.

- Symmetry: $D_{2 \mathrm{~h}}$.

- Basis set: ANO-RCC, contractions are [7s6p5d3f2g1h] for Fe, [4s3p2d1f] for C, N; and $[3 \mathrm{~s} 1 \mathrm{p}]$ for $\mathrm{H}$.

- Cholesky decomposition technique for the two-electron integrals with a threshold of $10^{-6}$ a u

- Scalar relativistic effect with standard second-order Douglas-Kroll-Hess (DKH) Hamiltonian.

- DMRG calculations: Fiedler orbital ordering, perturbative noise (noise prefactor of 0.05), residual norm threshold $10^{-7}$ for the Davidson algorithm.

- CASPT2 calculations: standard ionization potential electron affinity (IPEA) shift of $0.25 E_{\mathrm{h}}$, imaginary shift of $0.1 E_{\mathrm{h}}$. All valence electrons (but not the Fe $3 \mathrm{~s}$ and 3 p electrons) were correlated.

Table S1: Total energies (in $E_{\mathrm{h}}$ ) obtained from CASSCF/CASPT2 and DMRG/CASPT2 $[m=10000]$.

\begin{tabular}{ccccc}
\hline & CASSCF $^{a}$ & CASPT2 $^{a}$ & DMRG $^{b}$ & DMRG-CASPT2 $^{b}$ \\
\hline${ }^{3} \mathrm{~A}_{2 g}$ & -2254.391028 & -2258.538810 & -2254.556156 & -2258.506297 \\
${ }^{5} \mathrm{~A}_{1 g}$ & -2254.417218 & -2258.546312 & -2254.582257 & -2258.513704 \\
\hline
\end{tabular}




\section{Active orbitals of FeP}

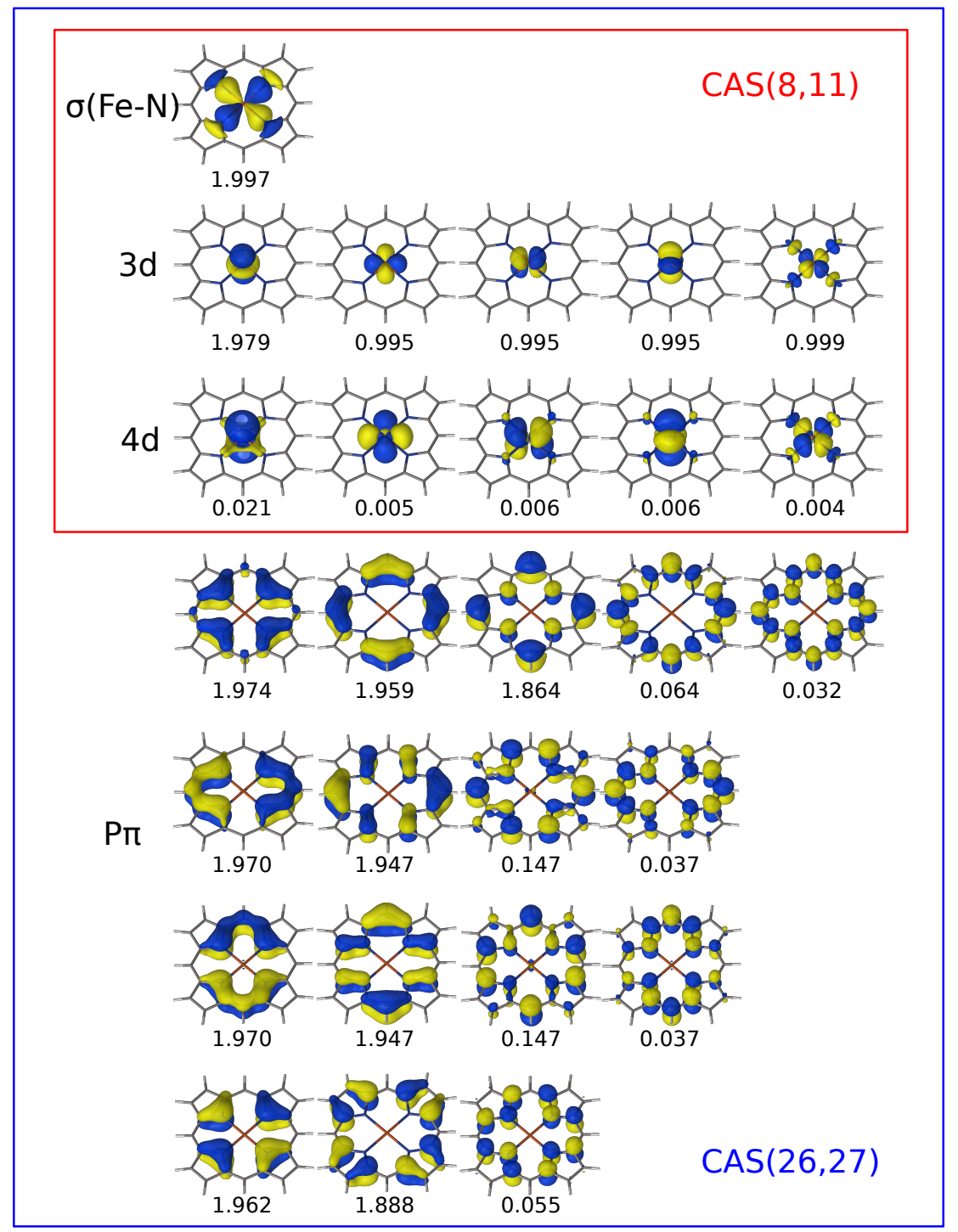

Figure S1: Active natural orbitals and natural orbital occupation numbers of ${ }^{5} \mathrm{~A}_{1 g} \mathrm{FeP}$. 


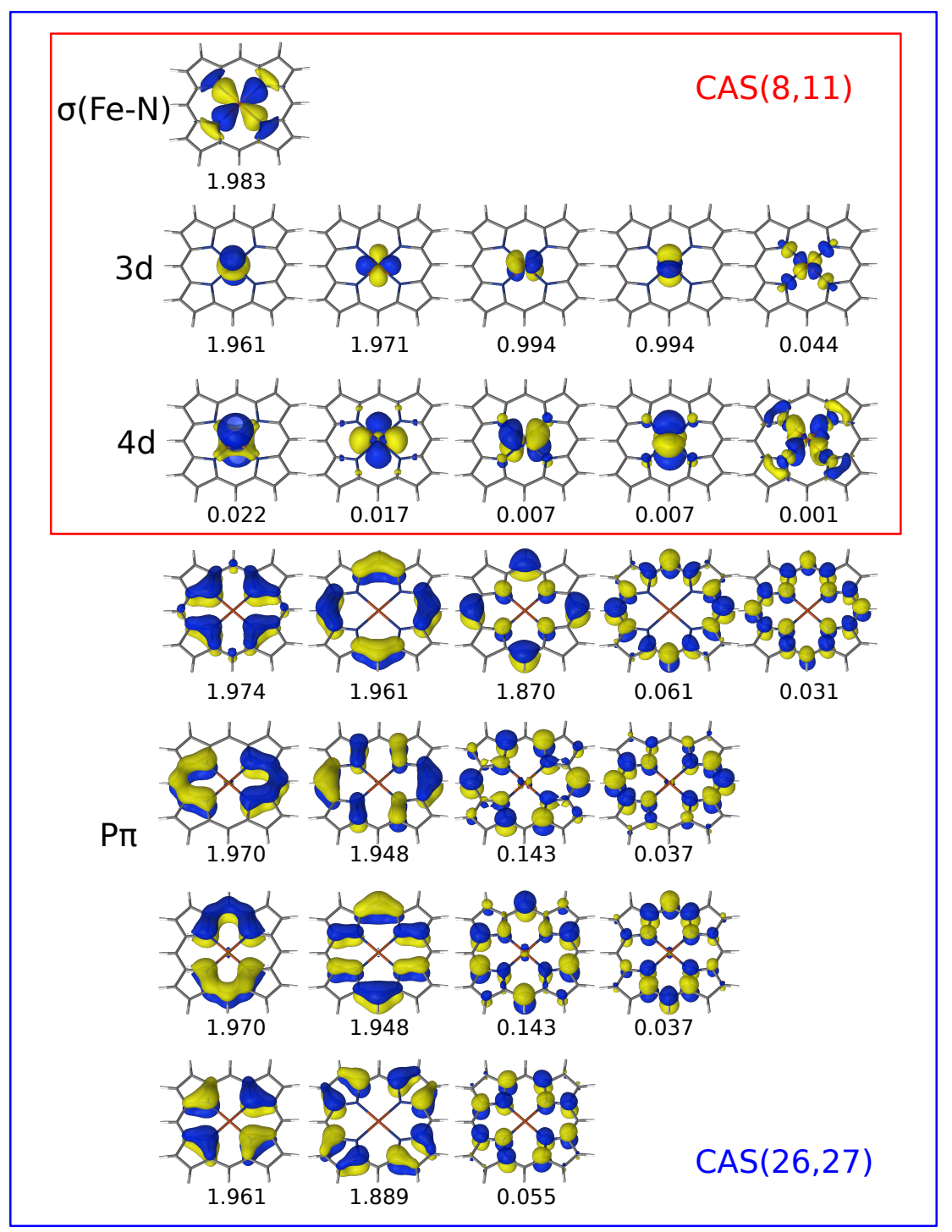

Figure S2: Active natural orbitals and natural orbital occupation numbers of ${ }^{3} \mathrm{~A}_{2 g}$ FeP.

\section{Cartesian coordinates of $\mathrm{FeP}$}

Table S2: PBE0/def2-TZVP structure (in $\AA$ ) of $\mathrm{FeP}^{3} \mathrm{~A}_{2 g}$.

\begin{tabular}{lrrr}
\multicolumn{3}{l}{37 atoms } \\
\hline $\mathrm{Fe}$ & 0.000000 & 0.000000 & 0.000000 \\
$\mathrm{~N}$ & 1.406727 & 1.406727 & 0.000000 \\
$\mathrm{~N}$ & -1.406727 & 1.406727 & 0.000000 \\
$\mathrm{~N}$ & 1.406727 & -1.406727 & 0.000000 \\
$\mathrm{~N}$ & -1.406727 & -1.406727 & 0.000000 \\
$\mathrm{C}$ & 0.000000 & 3.400142 & 0.000000 \\
$\mathrm{C}$ & 0.000000 & -3.400142 & 0.000000 \\
$\mathrm{C}$ & 3.400142 & 0.000000 & 0.000000 \\
$\mathrm{C}$ & -3.400142 & 0.000000 & 0.000000 \\
$\mathrm{C}$ & 2.483151 & 3.440698 & 0.000000 \\
$\mathrm{C}$ & -2.483151 & 3.440698 & 0.000000
\end{tabular}




\begin{tabular}{lrrr}
$\mathrm{C}$ & 2.483151 & -3.440698 & 0.000000 \\
$\mathrm{C}$ & -2.483151 & -3.440698 & 0.000000 \\
$\mathrm{C}$ & 3.440698 & 2.483151 & 0.000000 \\
$\mathrm{C}$ & -3.440698 & 2.483151 & 0.000000 \\
$\mathrm{C}$ & 3.440698 & -2.483151 & 0.000000 \\
$\mathrm{C}$ & -3.440698 & -2.483151 & 0.000000 \\
$\mathrm{C}$ & 1.222770 & 2.760387 & 0.000000 \\
$\mathrm{C}$ & -1.222770 & 2.760387 & 0.000000 \\
$\mathrm{C}$ & 1.222770 & -2.760387 & 0.000000 \\
$\mathrm{C}$ & -1.222770 & -2.760387 & 0.000000 \\
$\mathrm{C}$ & 2.760387 & 1.222770 & 0.000000 \\
$\mathrm{C}$ & -2.760387 & 1.222770 & 0.000000 \\
$\mathrm{C}$ & 2.760387 & -1.222770 & 0.000000 \\
$\mathrm{C}$ & -2.760387 & -1.222770 & 0.000000 \\
$\mathrm{H}$ & 0.000000 & 4.482672 & 0.000000 \\
$\mathrm{H}$ & 0.000000 & -4.482672 & 0.000000 \\
$\mathrm{H}$ & 4.482672 & 0.000000 & 0.000000 \\
$\mathrm{H}$ & -4.482672 & 0.000000 & 0.000000 \\
$\mathrm{H}$ & 4.513532 & 2.603261 & 0.000000 \\
$\mathrm{H}$ & -4.513532 & 2.603261 & 0.000000 \\
$\mathrm{H}$ & 4.513532 & -2.603261 & 0.000000 \\
$\mathrm{H}$ & -4.513532 & -2.603261 & 0.000000 \\
$\mathrm{H}$ & 2.603261 & 4.513532 & 0.000000 \\
$\mathrm{H}$ & -2.603261 & 4.513532 & 0.000000 \\
$\mathrm{H}$ & 2.603261 & -4.513532 & 0.000000 \\
$\mathrm{H}$ & -2.603261 & -4.513532 & 0.000000 \\
\hline & & &
\end{tabular}

Table S3: PBE0/def2-TZVP structure (in $\AA$ ) of FeP ${ }^{5} \mathrm{~A}_{1 g}$.

37 atoms

\begin{tabular}{lrrr}
\hline $\mathrm{Fe}$ & 0.000000 & 0.000000 & 0.000000 \\
$\mathrm{~N}$ & 1.451412 & 1.451412 & 0.000000 \\
$\mathrm{~N}$ & -1.451412 & 1.451412 & 0.000000 \\
$\mathrm{~N}$ & 1.451412 & -1.451412 & 0.000000 \\
$\mathrm{~N}$ & -1.451412 & -1.451412 & 0.000000 \\
$\mathrm{C}$ & 0.000000 & 3.417223 & 0.000000 \\
$\mathrm{C}$ & 0.000000 & -3.417223 & 0.000000 \\
$\mathrm{C}$ & 3.417223 & 0.000000 & 0.000000 \\
$\mathrm{C}$ & -3.417223 & 0.000000 & 0.000000 \\
$\mathrm{C}$ & 2.513686 & 3.473694 & 0.000000 \\
$\mathrm{C}$ & -2.513686 & 3.473694 & 0.000000 \\
$\mathrm{C}$ & 2.513686 & -3.473694 & 0.000000 \\
$\mathrm{C}$ & -2.513686 & -3.473694 & 0.000000 \\
$\mathrm{C}$ & 3.473694 & 2.513686 & 0.000000 \\
$\mathrm{C}$ & -3.473694 & 2.513686 & 0.000000 \\
$\mathrm{C}$ & 3.473694 & -2.513686 & 0.000000 \\
$\mathrm{C}$ & -3.473694 & -2.513686 & 0.000000 \\
$\mathrm{C}$ & 1.245363 & 2.796785 & 0.000000
\end{tabular}




\begin{tabular}{lrrr}
$\mathrm{C}$ & -1.245363 & 2.796785 & 0.000000 \\
$\mathrm{C}$ & 1.245363 & -2.796785 & 0.000000 \\
$\mathrm{C}$ & -1.245363 & -2.796785 & 0.000000 \\
$\mathrm{C}$ & 2.796785 & 1.245363 & 0.000000 \\
$\mathrm{C}$ & -2.796785 & 1.245363 & 0.000000 \\
$\mathrm{C}$ & 2.796785 & -1.245363 & 0.000000 \\
$\mathrm{C}$ & -2.796785 & -1.245363 & 0.000000 \\
$\mathrm{H}$ & 0.000000 & 4.500641 & 0.000000 \\
$\mathrm{H}$ & 0.000000 & -4.500641 & 0.000000 \\
$\mathrm{H}$ & 4.500641 & 0.000000 & 0.000000 \\
$\mathrm{H}$ & -4.500641 & 0.000000 & 0.000000 \\
$\mathrm{H}$ & 4.545432 & 2.645246 & 0.000000 \\
$\mathrm{H}$ & -4.545432 & 2.645246 & 0.000000 \\
$\mathrm{H}$ & 4.545432 & -2.645246 & 0.000000 \\
$\mathrm{H}$ & -4.545432 & -2.645246 & 0.000000 \\
$\mathrm{H}$ & 2.645246 & 4.545432 & 0.000000 \\
$\mathrm{H}$ & -2.645246 & 4.545432 & 0.000000 \\
$\mathrm{H}$ & 2.645246 & -4.545432 & 0.000000 \\
$\mathrm{H}$ & -2.645246 & -4.545432 & 0.000000 \\
\hline
\end{tabular}

\section{Convergence and performance}

In fig. [S3, we present the error of the quintet-triplet relative energy calculated at different $m$ values, using the data at $m=10000$ as a reference. The results show that both DMRGSCF and DMRG-CASPT2 results converge quickly with $m$. Even at the smallest $m$ value $(m=2000)$, the errors do not exceed $0.5 \mathrm{kcal} / \mathrm{mol}(2 \mathrm{~kJ} / \mathrm{mol})$. At $m=5000-6000$, the relative energies have already converged to within $0.1 \mathrm{kcal} / \mathrm{mol}(0.4 \mathrm{~kJ} / \mathrm{mol})$.

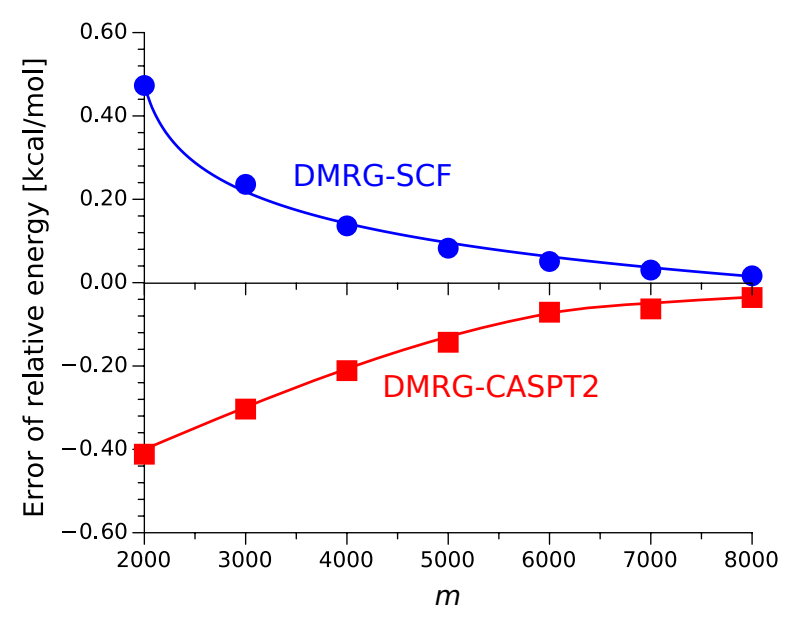

Figure S3: Error of the ${ }^{3} \mathrm{~A}_{2 g}-{ }^{5} \mathrm{~A}_{1 g}$ relative energy $\Delta E$ calculated at different $m$. The results at $m=10000$ are used as references, $\Delta E(\mathrm{DMRG}-\mathrm{SCF})=-68.53 \mathrm{~kJ} / \mathrm{mol}$, $\Delta E(\mathrm{DMRG}-\mathrm{CASPT} 2)=-19.46 \mathrm{~kJ} / \mathrm{mol}$. All valence electrons (but not Fe (3s, 3p) were correlated in the PT2 treatment.

In fig. [S4, we report the computing time of the F.4-RDM and 3-RDM, the most time consuming part of these calculations, as well as the required computational resources (memory and temporary disk space). All calculations were done on a single node with an 
Intel(R) Xeon(R) CPU 6140 @ 2.3 GHz, $768 \mathrm{~GB}$ of memory, and 4 MPI processes. The computational complexity of the DMRG-CASPT2 calculations and the required computer resources grow quickly, though polynomially, with the number of active orbitals $n$ and number of renormalized states $m \cdot \underline{\left[\mathrm{S}_{\mathrm{S} 2} \mathrm{~S}\right.}$ At $m=10000$, the calculations demand almost 120 hours computing time for the F.4-RDM (fig. S4(a)), a very large amount of RAM (up to $700 \mathrm{~GB}$ ) and modest temporary disk space (see fig. S4(b)). However as shown above, one does not have to raise $m$ to 10000 in order to achieve converged results. In the present case, $m=5000-6000$, requiring only moderate computational resources, already provides converged results to $\sim 0.4 \mathrm{~kJ} / \mathrm{mol}$.
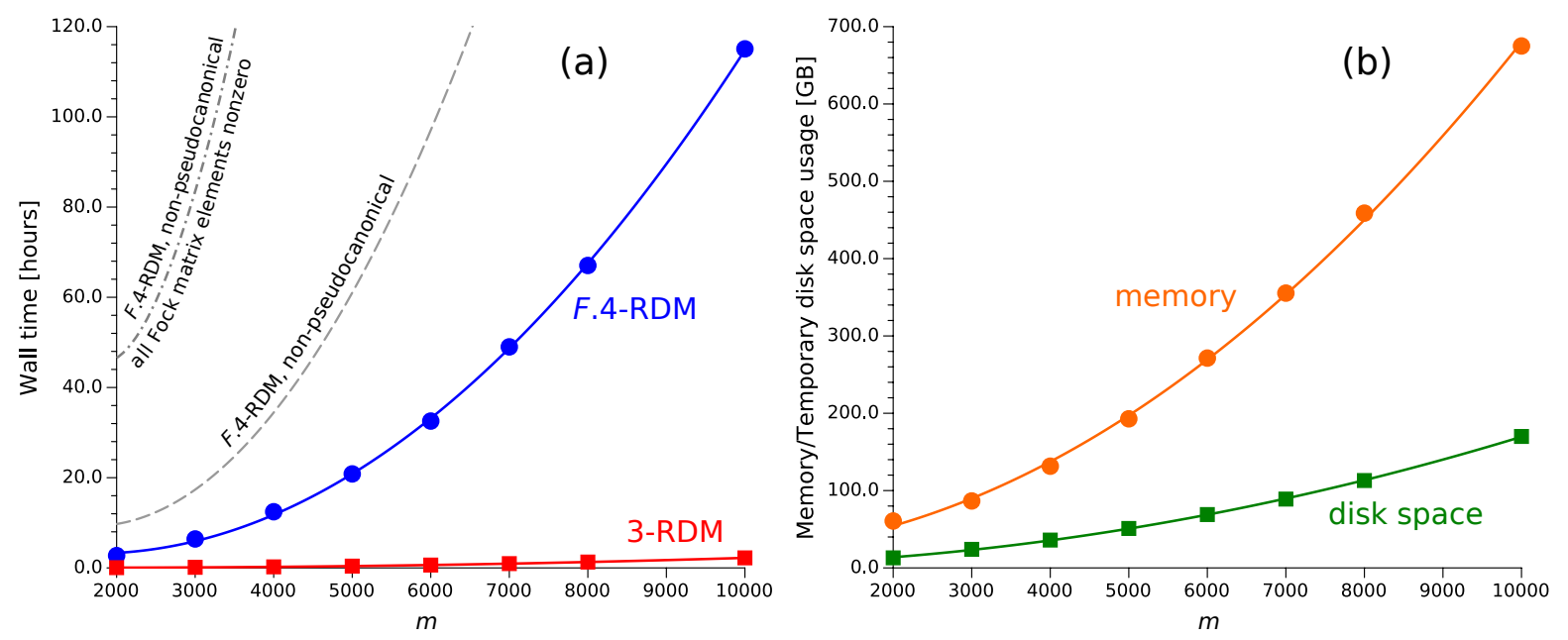

Figure S4: (a) Computing time (in hours) of the reduced density matrices (3-RDM and F.4-RDM) using pseudocanonical orbital basis and (b) computational resources needed (memory and disk storage) (in GB) at different number of renormalized states $m$. The data in gray lines are estimated based on the number of nonzero Fock matrix elements: dashed line - block diagonal Fock matrix; dash-dotted line - all Fock matrix elements are nonzero.

Finally, we illustrate the advantage of using the pseudocanonical orbital basis (diagonal Fock matrix) in the calculation of the F.4-RDM (fig. S4(a)). The wall-time of F.4-RDM is proportional to the number of nonzero Fock matrix elements.

For pseudocanonical orbitals, it only scales as $\mathcal{O}\left(n^{5} m^{3}+n^{7} m^{2}\right)$ (with $n$ the number of active orbitals, see also fig. S5) and is almost 3 times smaller than using a nonpseudocanonical orbital basis (block diagonal Fock matrix, gray dashed line). Without molecular symmetry, all Fock matrix elements have to be considered (gray dash-dotted line), and we expect a significantly longer wall-time to calculate the F.4-RDM. 


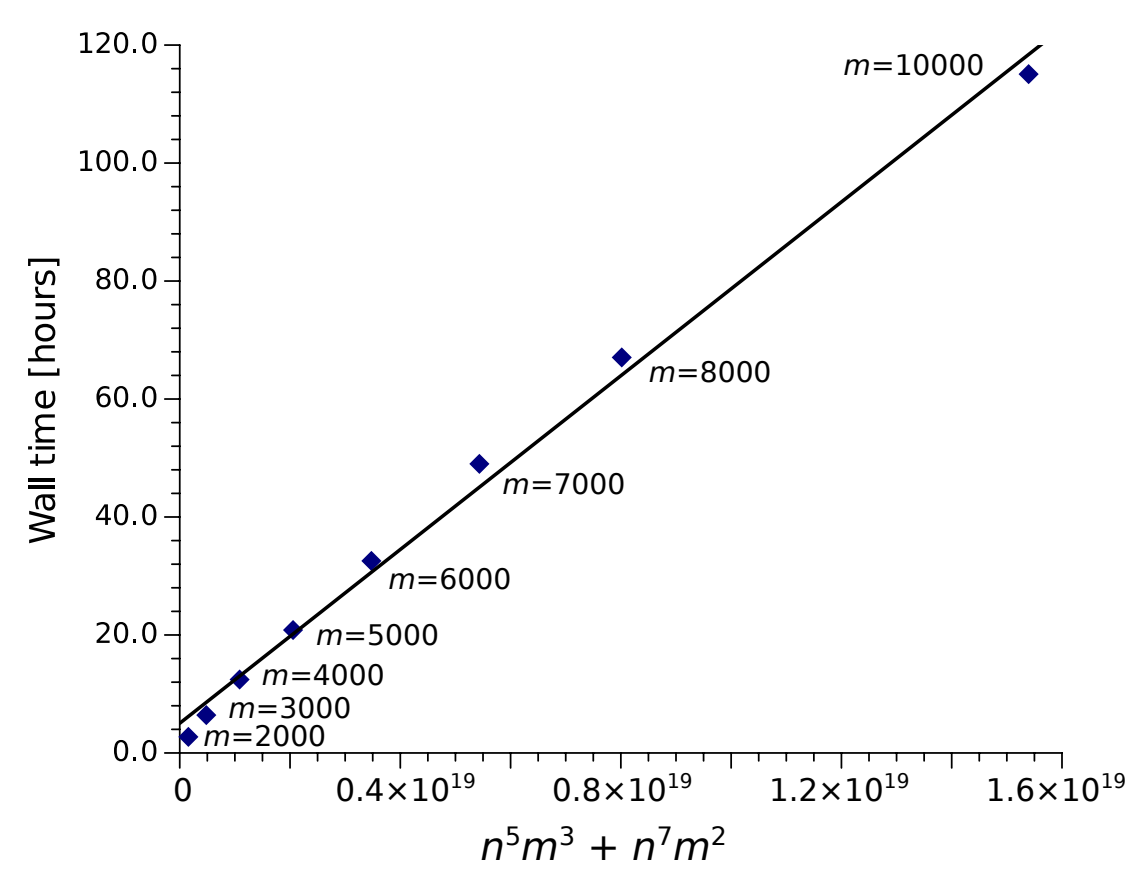

Figure S5: Computing time (in hours) for the F.4-RDM as a function of $n^{5} m^{3}+n^{7} m^{2}$, $n=27$.

\section{Equilibrium structure of ferrocene}

\section{Computational details}

- Active space: CAS $(10,10)$ and $\operatorname{CAS}(14,18)$, see fig. S6.

- Cartesian coordinates optimized with CASPT2 and DMRG-CASPT2, see tables S4 and $[55$

- Symmetry: $C_{2 v}$.

- Basis set: cc-pwCVTZ, is the same as used before in the work of Harding et al. $\underline{\text { [3] }}$

- Cholesky decomposition technique for the two-electron integrals with a threshold of $10^{-6} E_{\mathrm{h}}$

- DMRG calculations: $m=1000$, Fiedler orbital ordering, perturbative noise (noise prefactor of 0.05), residual norm threshold $10^{-4}$ for the Davidson algorithm.

- CASPT2 calculations: standard ionization potential electron affinity (IPEA) shift of $0.25 E_{\mathrm{h}}$, imaginary shift of $0.1 E_{\mathrm{h}}$. All 96 electrons were correlated, which was used in the work of Harding et al. $\underline{\text { S3 }}$

- NUMERICAL_GRADIENT module: the number of displacements is 32 .

- SLAPAF module: Convergence criteria with respect to the energy change and the norm of the gradient are $1.0 \times 10^{-5} E_{\mathrm{h}}$ and $1.0 \times 10^{-3} E_{\mathrm{h}} / a_{0}$, respectively. 
Active orbitals of ferrocene
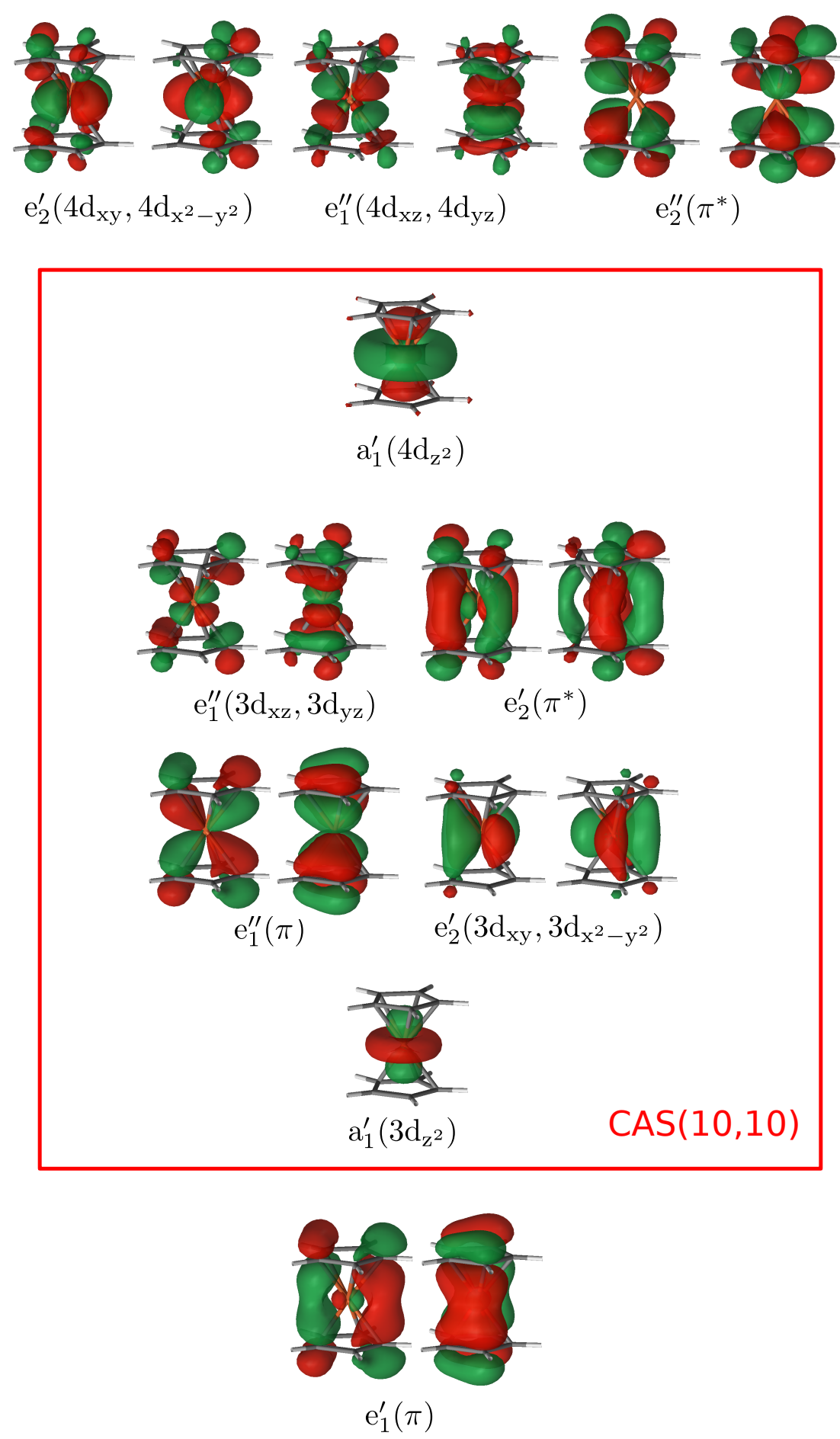

Figure S6: Active natural orbitals of ferrocene.

\section{Cartesian coordinates of ferrocene}

Table S4: Equilibrium structure (in $\AA$ ) of ferrocene calculated with CASPT2.

21 atoms

Fe $\quad 0.00000000 \quad-0.00082875 \quad 0.00000000$




\begin{tabular}{lrrr}
$\mathrm{C}$ & 0.00000000 & 1.21280272 & 1.60955791 \\
$\mathrm{C}$ & 1.15393751 & 0.37439976 & 1.60971190 \\
$\mathrm{C}$ & 0.71318536 & -0.98218137 & 1.60996993 \\
$\mathrm{H}$ & 0.00000000 & 2.28932149 & 1.59605780 \\
$\mathrm{H}$ & 2.17770932 & 0.70713134 & 1.59589850 \\
$\mathrm{H}$ & 1.34593205 & -1.85309725 & 1.59641861 \\
$\mathrm{C}$ & 0.00000000 & 1.21280272 & -1.60955791 \\
$\mathrm{C}$ & -1.15393751 & 0.37439976 & -1.60971190 \\
$\mathrm{C}$ & 1.15393751 & 0.37439976 & -1.60971190 \\
$\mathrm{C}$ & -1.15393751 & 0.37439976 & 1.60971190 \\
$\mathrm{C}$ & -0.71318536 & -0.98218137 & -1.60996993 \\
$\mathrm{C}$ & 0.71318536 & -0.98218137 & -1.60996993 \\
$\mathrm{C}$ & -0.71318536 & -0.98218137 & 1.60996993 \\
$\mathrm{H}$ & 0.00000000 & 2.28932149 & -1.59605780 \\
$\mathrm{H}$ & -2.17770932 & 0.70713134 & -1.59589850 \\
$\mathrm{H}$ & 2.17770932 & 0.70713134 & -1.59589850 \\
$\mathrm{H}$ & -2.17770932 & 0.70713134 & 1.59589850 \\
$\mathrm{H}$ & -1.34593205 & -1.85309725 & -1.59641861 \\
$\mathrm{H}$ & 1.34593205 & -1.85309725 & -1.59641861 \\
$\mathrm{H}$ & -1.34593205 & -1.85309725 & 1.59641861 \\
\hline
\end{tabular}

Table S5: Equilibrium structure (in $\AA$ ) of ferrocene calculated with DMRG-CASPT2.

\begin{tabular}{lrrr}
\multicolumn{4}{l}{21 atoms } \\
\hline $\mathrm{Fe}$ & 0.00000000 & -0.00083682 & 0.00000000 \\
$\mathrm{C}$ & 0.00000000 & 1.21275757 & 1.61488082 \\
$\mathrm{C}$ & 1.15409820 & 0.37399205 & 1.61543911 \\
$\mathrm{C}$ & 0.71313728 & -0.98218300 & 1.61540035 \\
$\mathrm{H}$ & 0.00000000 & 2.28872950 & 1.59991555 \\
$\mathrm{H}$ & 2.17716570 & 0.70742566 & 1.59888643 \\
$\mathrm{H}$ & 1.34589032 & -1.85233701 & 1.59823707 \\
$\mathrm{C}$ & 0.00000000 & 1.21275757 & -1.61488082 \\
$\mathrm{C}$ & -1.15409820 & 0.37399205 & -1.61543911 \\
$\mathrm{C}$ & 1.15409820 & 0.37399205 & -1.61543911 \\
$\mathrm{C}$ & -1.15409820 & 0.37399205 & 1.61543911 \\
$\mathrm{C}$ & -0.71313728 & -0.98218300 & -1.61540035 \\
$\mathrm{C}$ & 0.71313728 & -0.98218300 & -1.61540035 \\
$\mathrm{C}$ & -0.71313728 & -0.98218300 & 1.61540035 \\
$\mathrm{H}$ & 0.00000000 & 2.28872950 & -1.59991555 \\
$\mathrm{H}$ & -2.17716570 & 0.70742566 & -1.59888643 \\
$\mathrm{H}$ & 2.17716570 & 0.70742566 & -1.59888643 \\
$\mathrm{H}$ & -2.17716570 & 0.70742566 & 1.59888643 \\
$\mathrm{H}$ & -1.34589032 & -1.85233701 & -1.59823707 \\
$\mathrm{H}$ & 1.34589032 & -1.85233701 & -1.59823707 \\
$\mathrm{H}$ & -1.34589032 & -1.85233701 & 1.59823707 \\
\hline
\end{tabular}




\section{Computational details for the DMRG-NEVPT2 example}

The Cartesian coordinates for all three states were the same and were taken from ref. S4. The all-electron ANO-RCC $\sqrt{\text { S5 }}$ basis set with the valence quadruple-zeta polarized (ANO-RCC-VQZP) contraction scheme was chosen for the Fe atom and the valence double-zeta polarized (ANO-RCC-VDZP) contraction scheme for other atoms, resulting in a total of 1595 basis functions. Two-electron integrals were calculated with the atomic compact Cholesky decomposition approach (acCD) w6 $^{\mathbf{S}}$ with a decomposition threshold of $10^{-4} E_{\mathrm{h}}$. Scalar-relativistic effects were accounted for through the second-order scalarrelativistic Douglas-Kroll-Hess one-electron Hamiltonian. $\underline{\text { S9 }}$ S11

\section{Multiconfiguration pair-density functional theory. Input examples}

\section{General input for single-point MC-PDFT calculations}

MC-PDFT calculation of the $\mathrm{N}_{2}$ molecule using tPBE, ftPBE, and tOPBE:

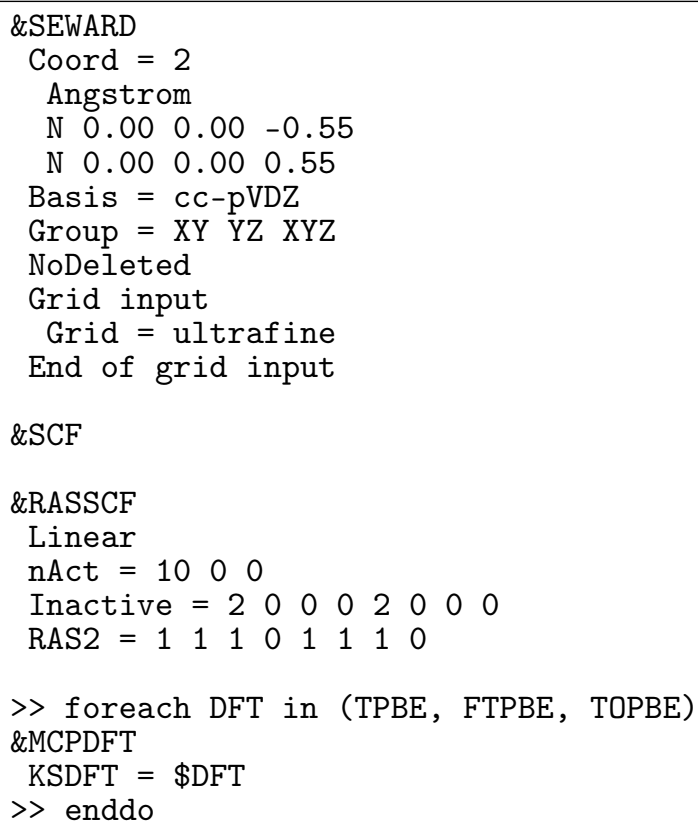

\section{SS-CAS-PDFT gradient}

Geometry optimization of the $\mathrm{N}_{2}$ molecule using SS-CAS-PDFT with the tPBE on-top functional:

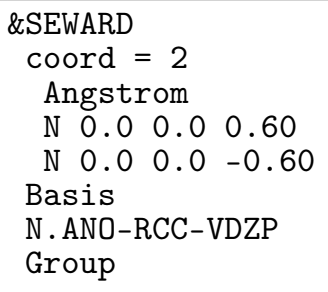




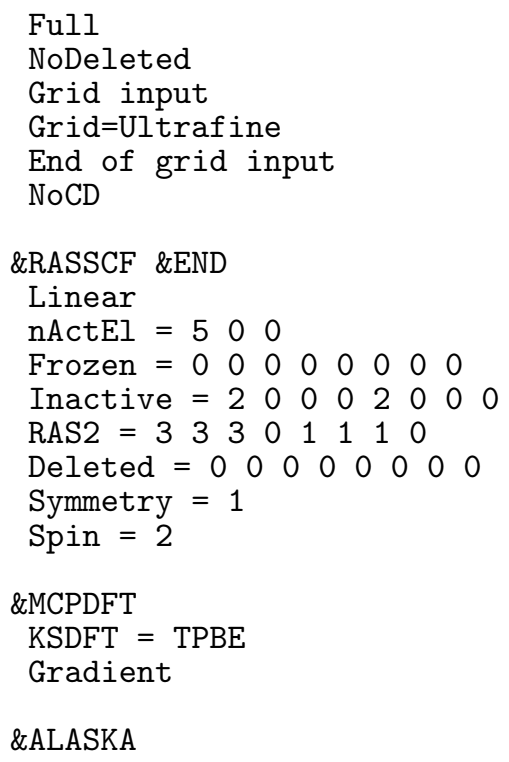

\&ALASKA

\section{Scaling exchange and correlation terms in MC-PDFT and KS-DFT}

Geometry optimization of the $\mathrm{N}_{2}$ molecule using SS-CAS-PDFT with an HLE-type functional constructed from scaling the exchange term and the correlation term of tPBE by 1.25 and 0.5 , respectively.

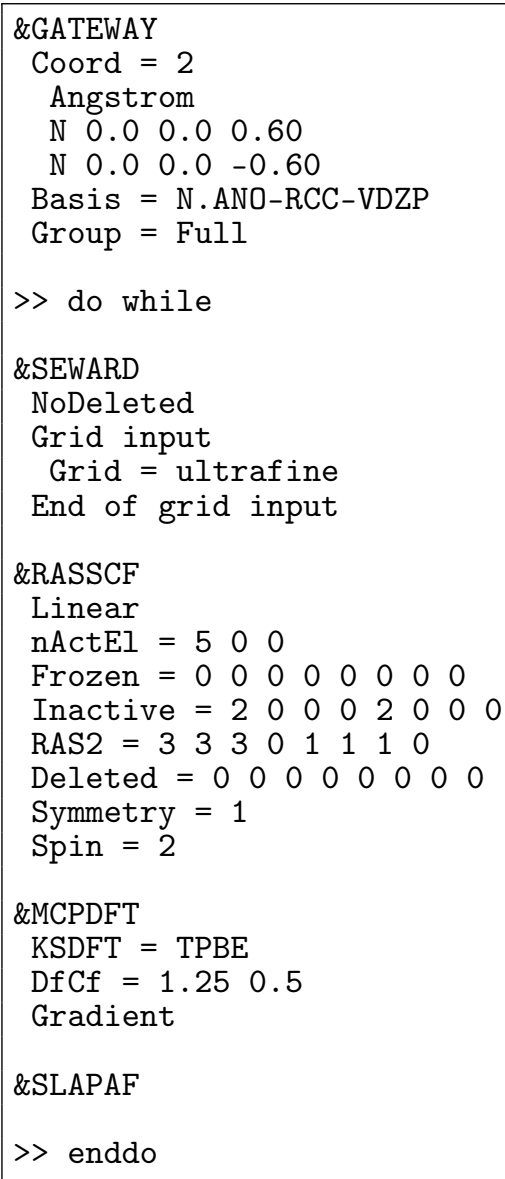


Kohn-Sham density functional theory calculation of the $\mathrm{N}_{2}$ molecule using the B3LYP functional with the correlation terms scaled by a factor of 0.8 .

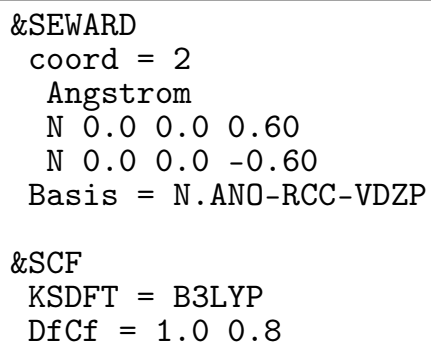

\section{SI-PDFT}

The following is an example SI-PDFT run for the dissociation of LiF:

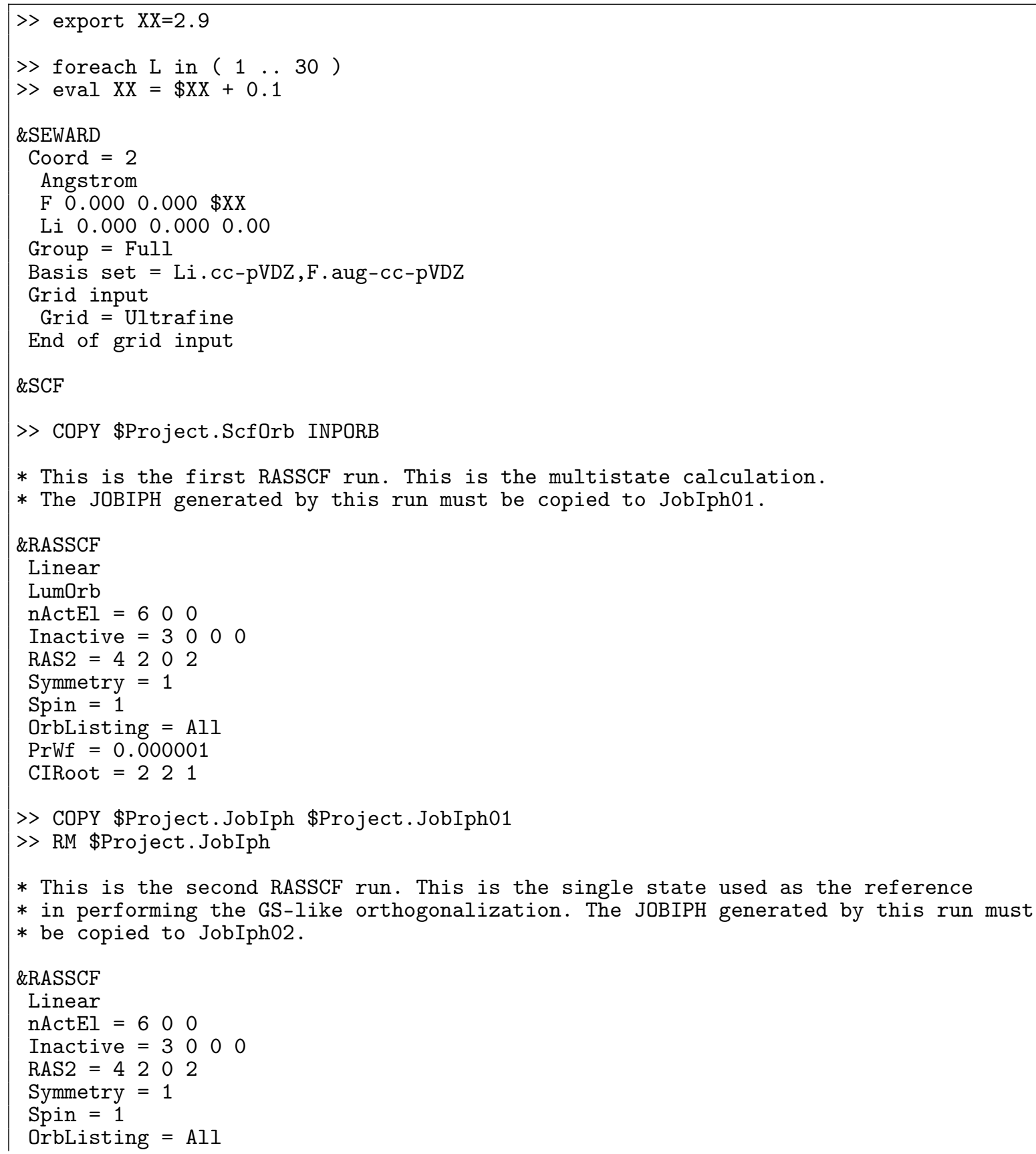




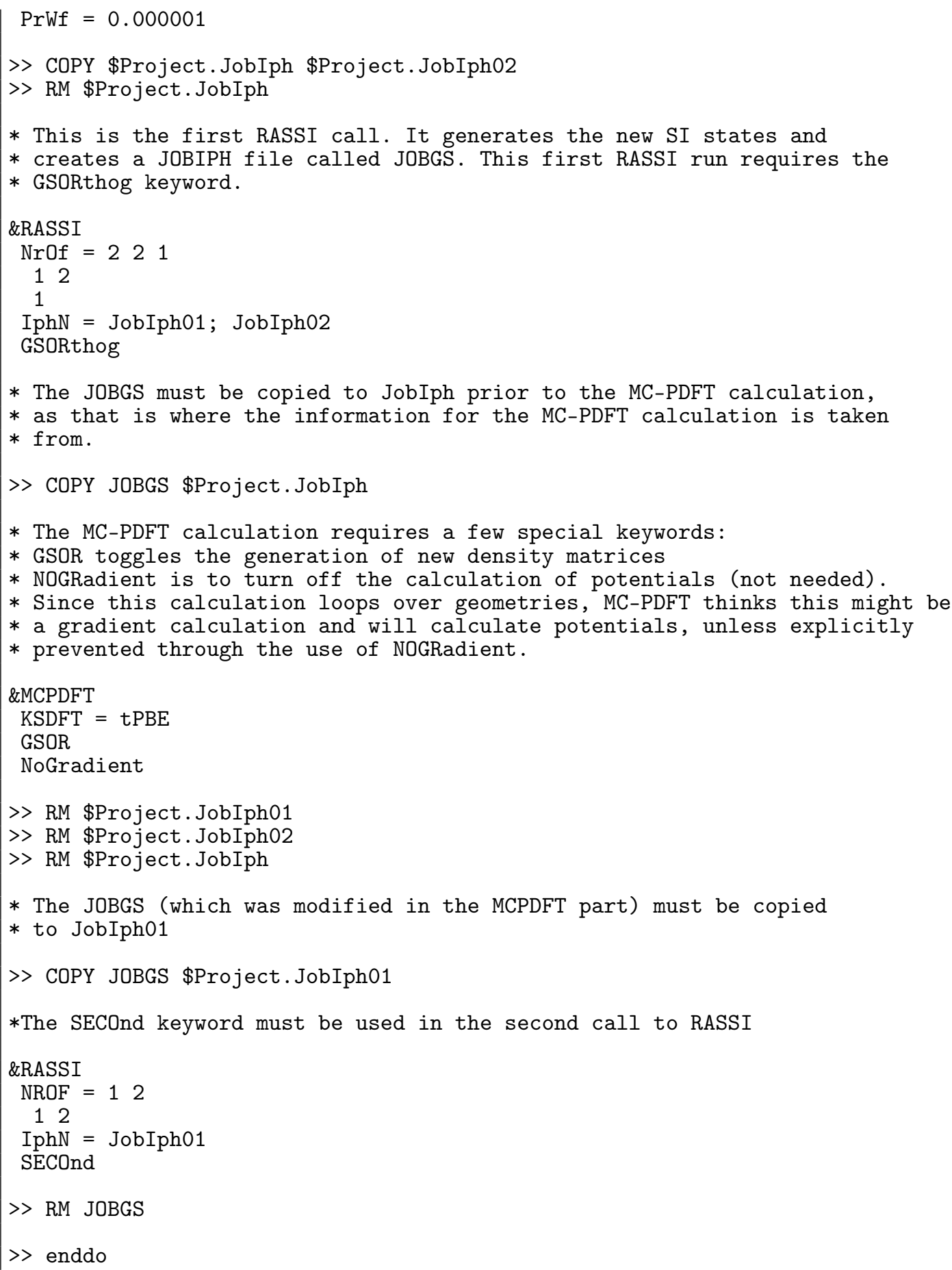

General input for a single-point DMRG-PDFT calculation

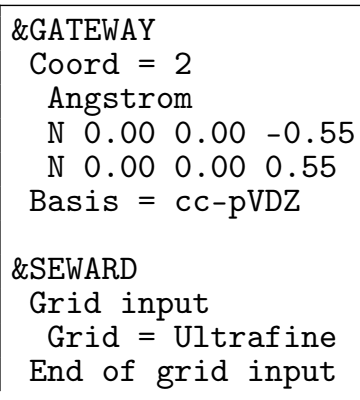




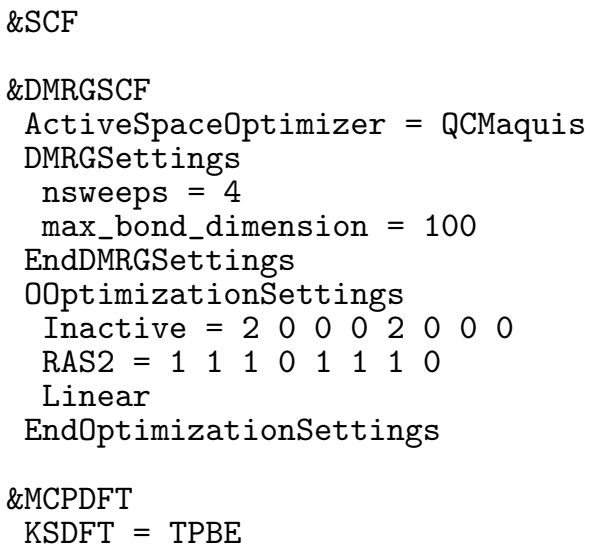

\section{Conical intersection optimization and characterization}

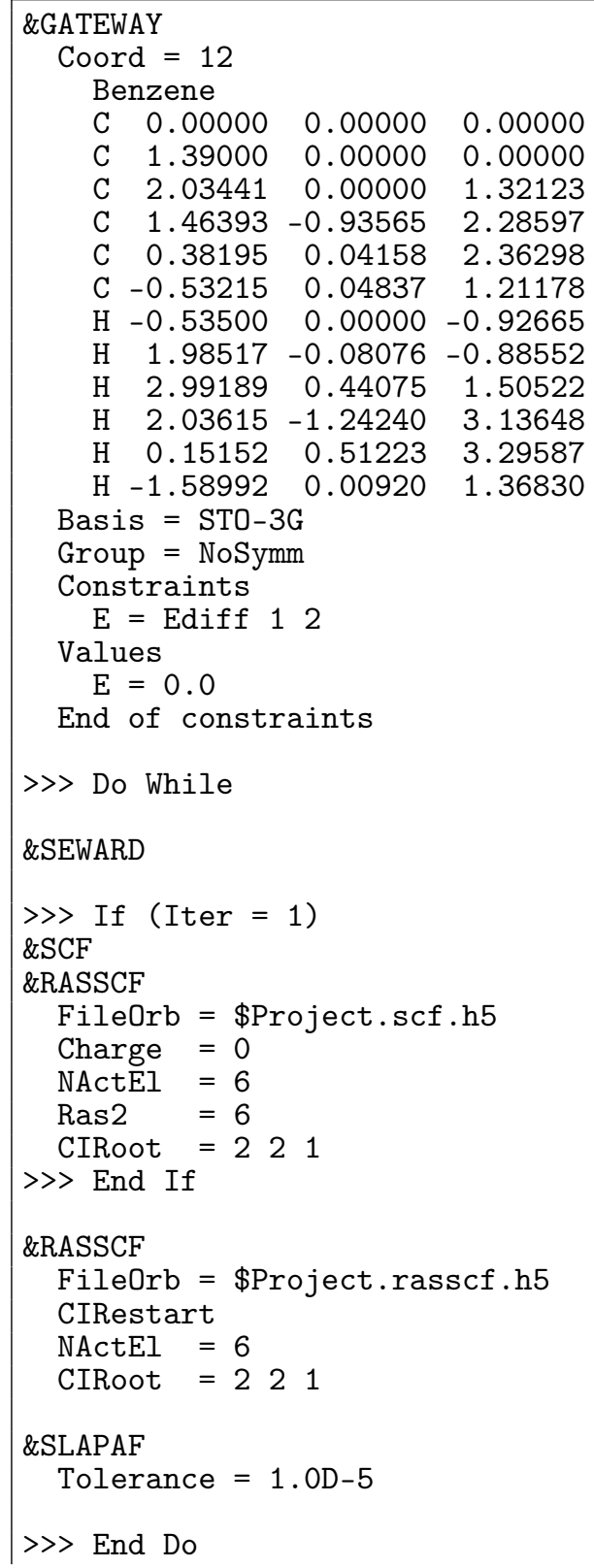


Listing S7: Input example for optimization of a conical intersection between the two lowest singlet states of benzene.

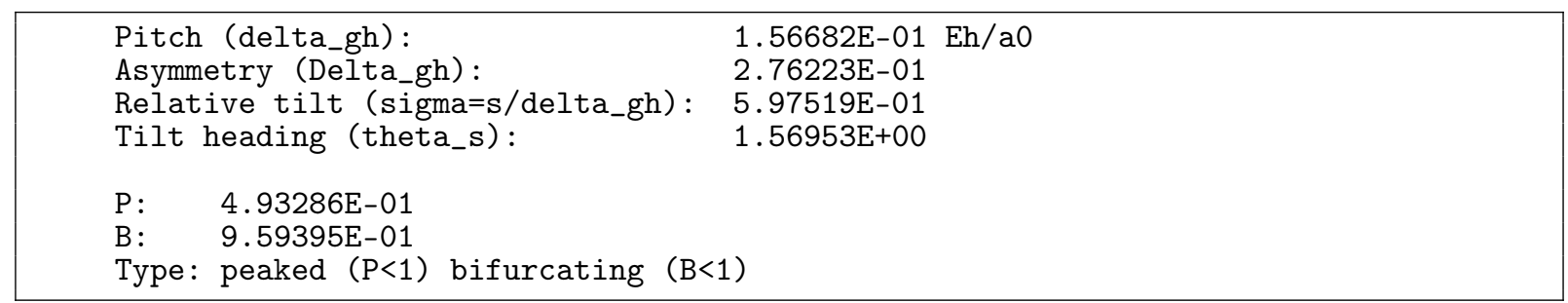

Listing S8: Characterization parameters at the converged structure from listing $S 7$.

\section{Setup for a single SHARC trajectory}

This quick tutorial presents how to setup a single SHARC trajectory using OpenMolcas as quantum chemistry program. To this end, we use the interface script \$SHARC/SHARC_MOLCAS.py, which fully supports OpenMolcas. Note that typically, SHARC trajectories are not setup manually, but using the tools of the SHARC suite. Hence, for a more complete overview over the SHARC suite, please see the manual and tutorial of SHARC at http://www.sharc-md.org/.

The files that need to be prepared and the relevant directory structure are presented in fig. S7. The contents of the files are given and explained in the following. Please be aware that the two subdirectories need to exist before SHARC can be started.

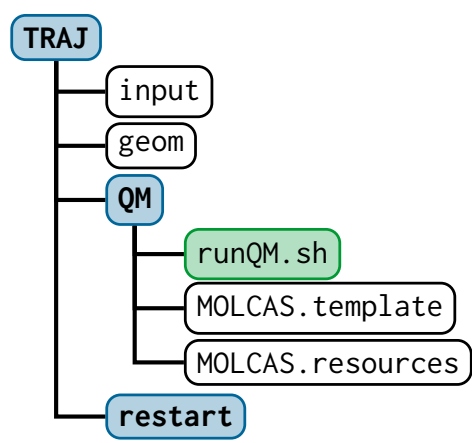

Figure S7: Input files for a SHARC dynamics simulation of a single, independent trajectory.

\section{Input file}

The SHARC input file ("input") contains the dynamics settings and names of additional input files (geometry, velocity, coefficients). An example is given below:

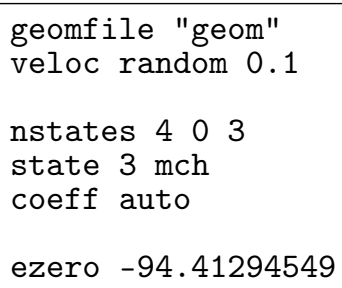




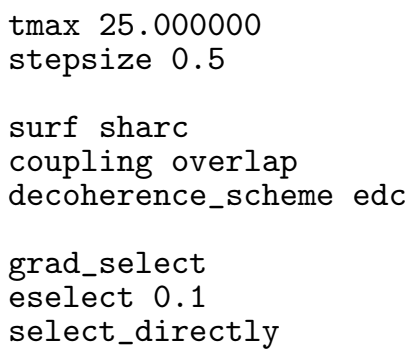

The meaning of these keywords is: The geometry is read from file geom. The nuclear velocities are picked at random, with $0.1 \mathrm{eV}$ kinetic energy per atom. Four singlet states and three triplet states will be included in the simulation $(0$ is the number of doublet states). The initial state is the third state (the $S_{2}$ ). The initial coefficients will be set automatically (the initial state will have a coefficient of 1.0, the remaining states a coefficient of zero). The diagonal elements of the Hamiltonian will be shifted by $-94.41294549 E_{\mathrm{h}}$. The simulation will run for $25 \mathrm{fs}$ with a $0.5 \mathrm{fs}$ timestep. The SHARC formalism will be used (propagation on the diagonalized states). Nonadiabatic interactions are described with wave function overlaps. SHARC will select which gradients to compute at each time step, with a selection threshold of $0.1 \mathrm{eV}$. SHARC will select these gradients directly, without doing two quantum chemistry calculations per time step.

\section{Geometry file}

The geometry file "geom" contains the chemical symbols, atomic charge, $x, y$ and $z$ coordinates and the relative atomic masses.

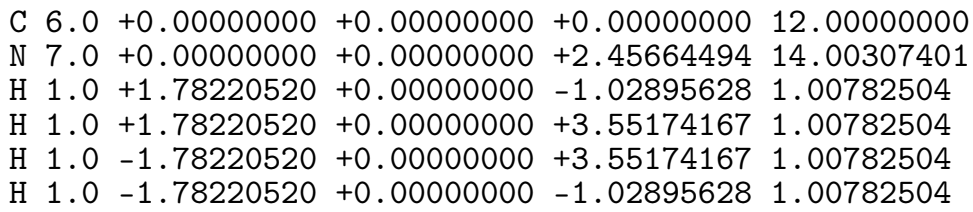

\section{QM run script}

At each timestep, SHARC writes the current geometry and different keywords to the file QM/QM. in and then calls runQM.sh. After this call is finished, SHARC reads the results of the quantum chemistry calculation from QM. out.

In most of the cases, in runQM . sh simply one of the SHARC-interfaces is called:

cd QM

\$SHARC/SHARC_MOLCAS.py QM.in >> QM.log 2>> QM.err

The interface will do all work necessary to produce the desired file QM. out.

\section{Template file}

The interface needs as additional input file giving the settings for the electronic structure calculation. The file is called "MOLCAS . template". It employs a simple keyword-argument structure and looks like:

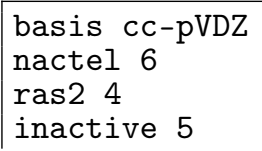




\section{Resource file}

The interface additionally needs some paths (e.g., to the OpenMolcas installation) and resource settings, both of which are read from the file "MOLCAS.resources".

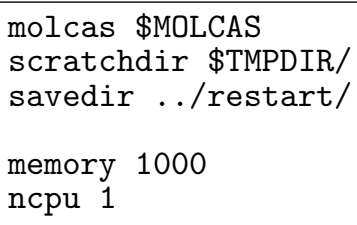

\section{Running SHARC}

With the input files prepared, the trajectory can then be started by simply executing:

\$SHARC/sharc.x input

\section{Output}

SHARC produces four output files, output.log, output.lis, output.dat and output.xyz. The file output.log contains mainly a listing of the chosen options and the resulting dynamics settings. At higher print levels, the log file contains also information per timestep. output. Iis contains a table with one line per timestep, giving active states, energies and expectation values. output. dat contains a list of all important matrices and vectors at each timestep. This information can be extracted with data_extractor. $\mathrm{x}$ to yield plottable table files. output.xyz contains the geometries of all timesteps, allowing visualization of the trajectory with the appropriate software (e.g., Molden).

\section{Light-matter interaction}

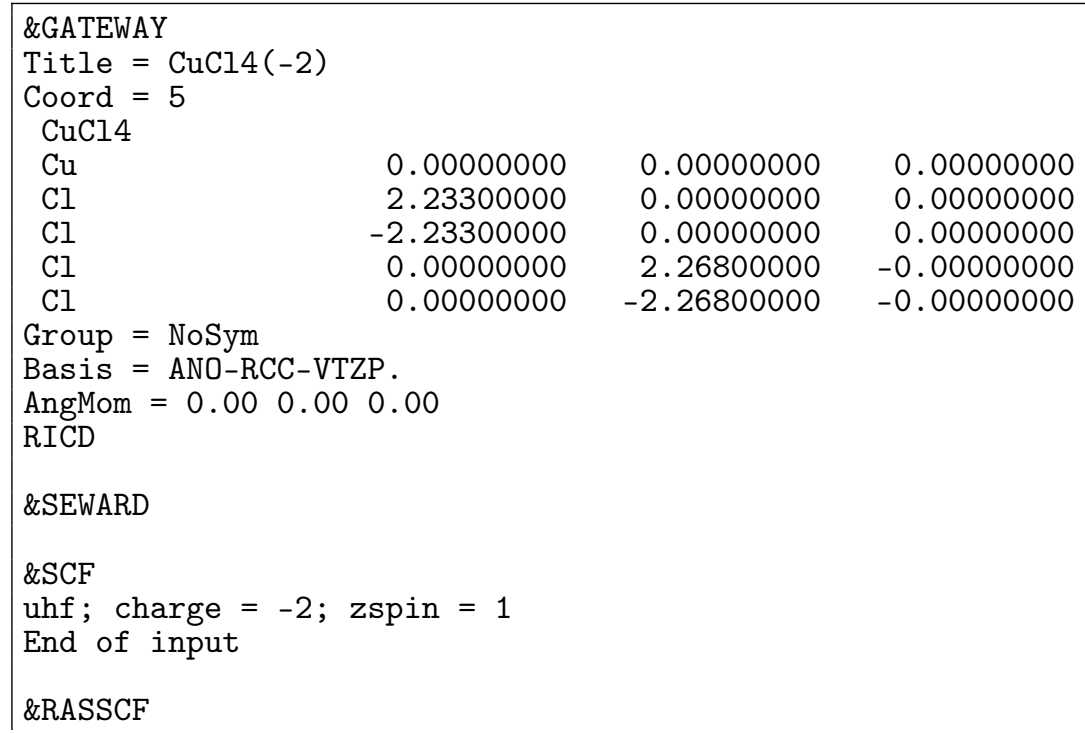




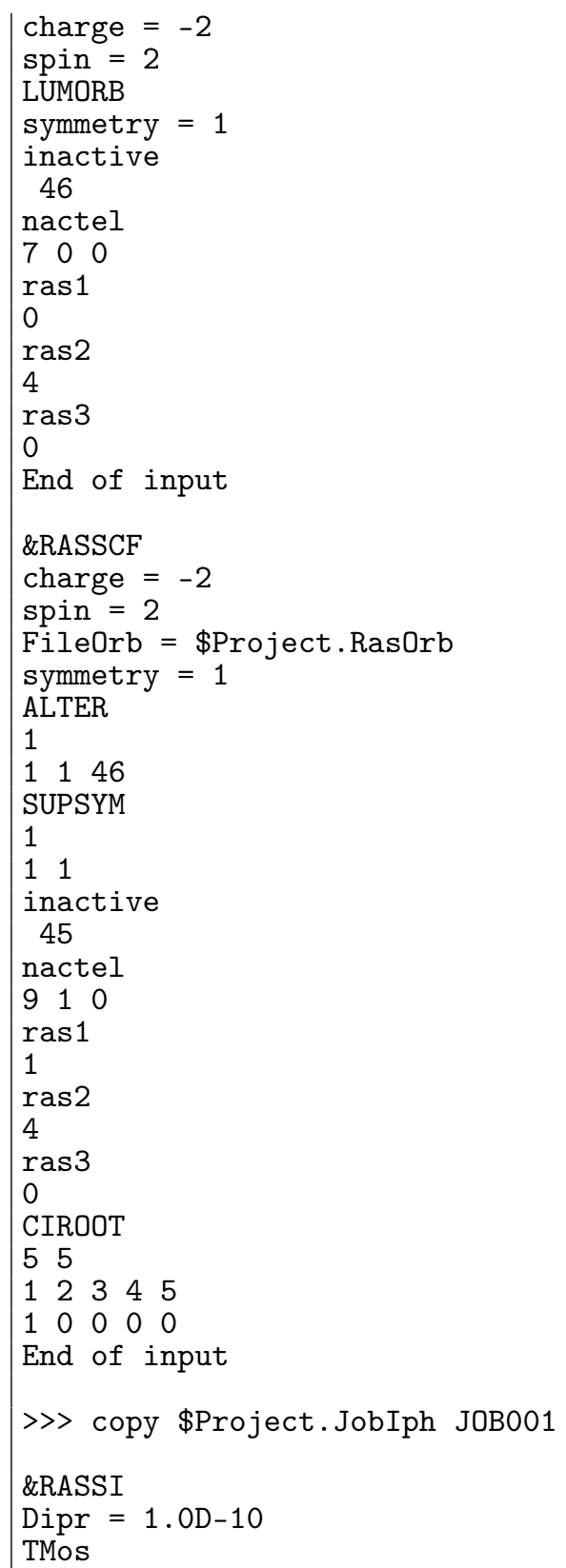

\begin{tabular}{|lrrr}
\hline \&GATEWAY & & \\
Title $=\operatorname{CuCl4}(-2)$ & & \\
Coord $=5$ & & & \\
CuCl4 & & & \\
Cu & 0.00000000 & 0.00000000 & 0.00000000 \\
Cl & 2.23300000 & 0.00000000 & 0.00000000 \\
Cl & -2.23300000 & 0.00000000 & 0.00000000 \\
Cl & 0.00000000 & 2.26800000 & -0.00000000 \\
Cl & 0.00000000 & -2.26800000 & -0.00000000
\end{tabular}

Group = NoSym

Basis = ANO-RCC-VTZP.

RICD

\&SEWARD

\&SCF

uhf; charge $=-2 ;$ zspin $=1$

End of input

\&RASSCF

charge $=-2$ 


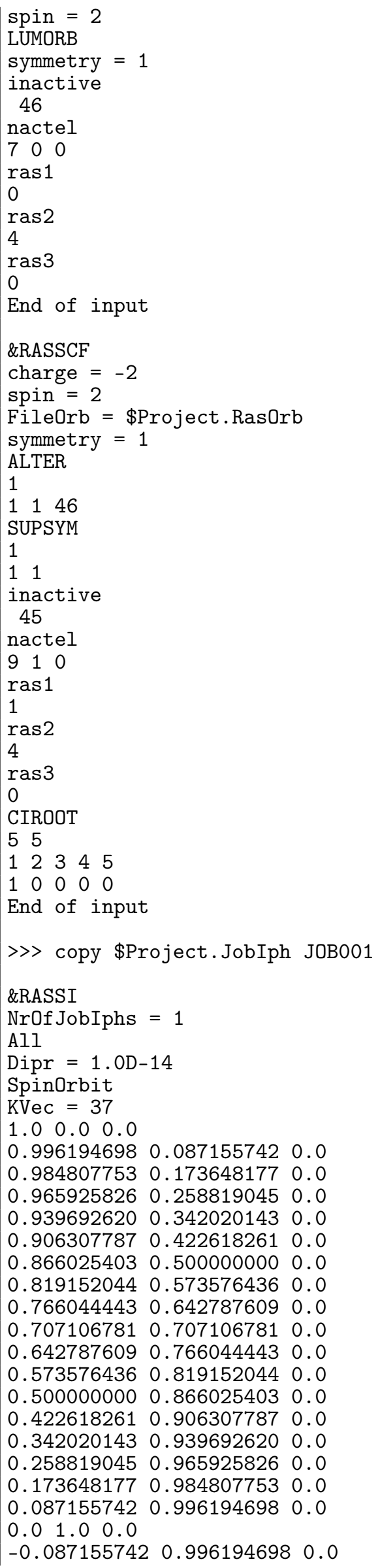




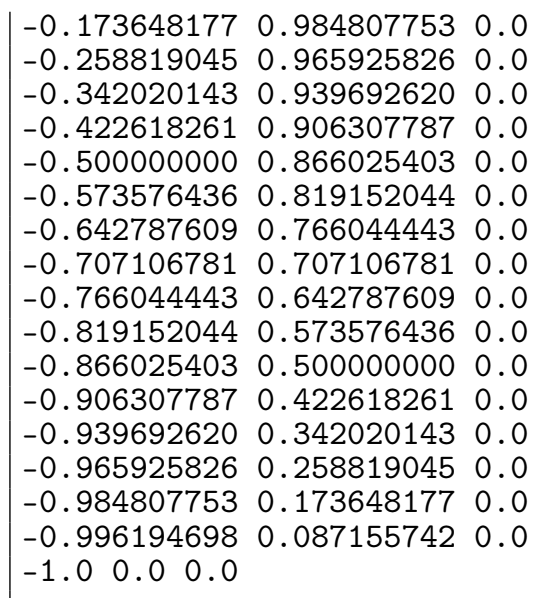

\section{Multi-configurational Dyson orbitals in RASSI}

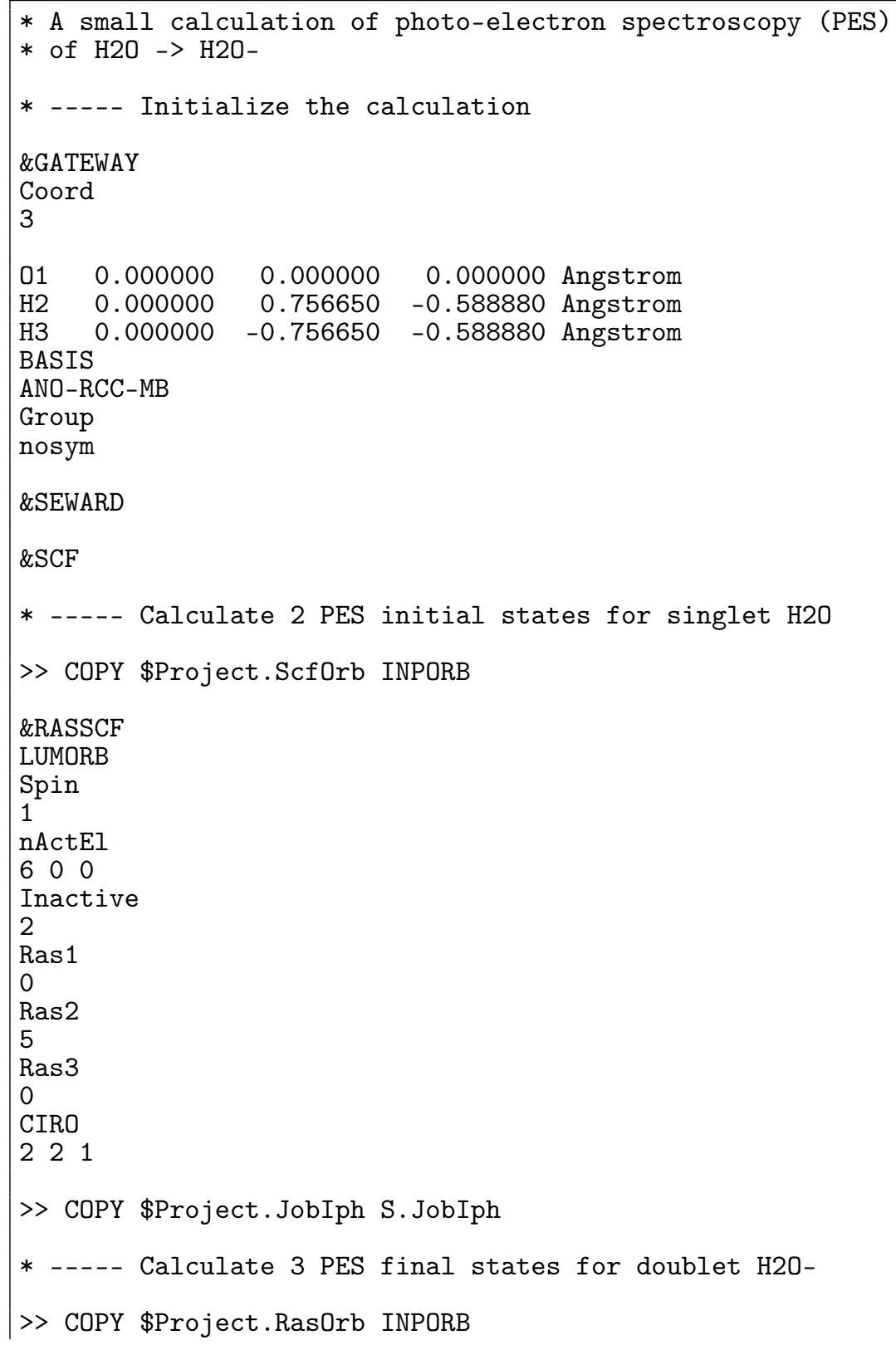




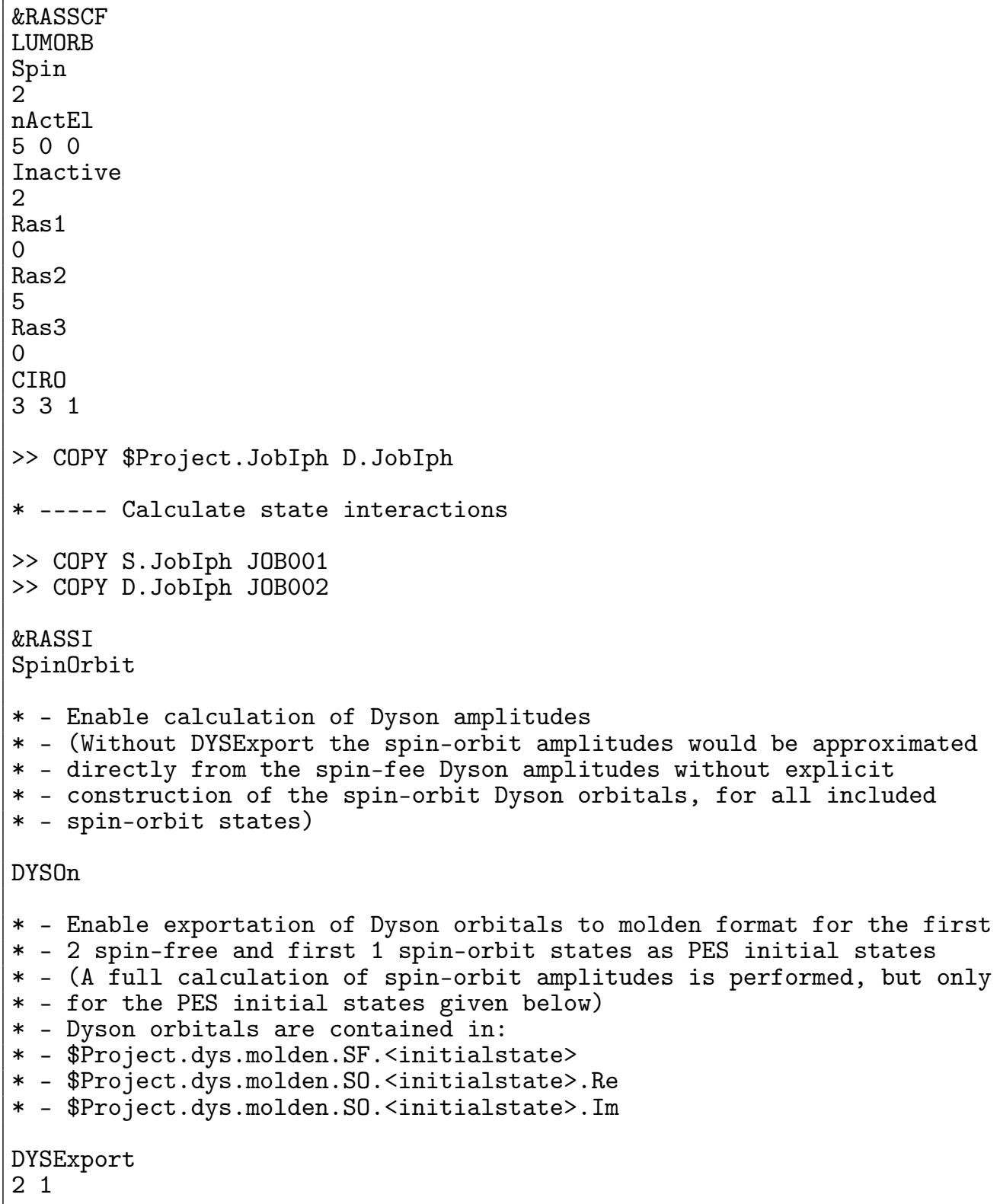

\section{Orbital properties}

\section{Printing orbital second moment $\left\langle r^{2}\right\rangle$ for all orbitals to identify Rydberg orbitals}

We take a trans-1,3-butadiene molecule as an example. First, generate at least one orbital file, such as the CASSCF natural orbitals (\$Project.RasOrb, \$Project.Ras0rb.1, \$Project.RasOrb.2, ...):

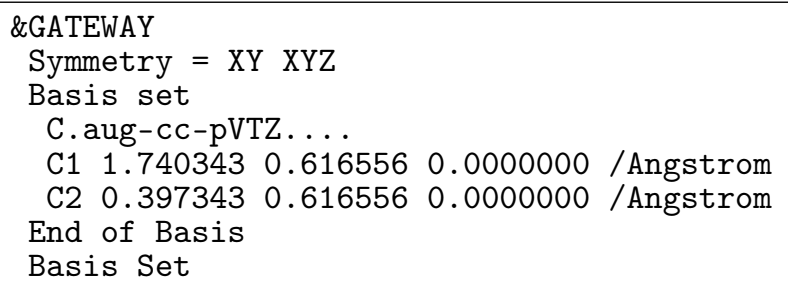




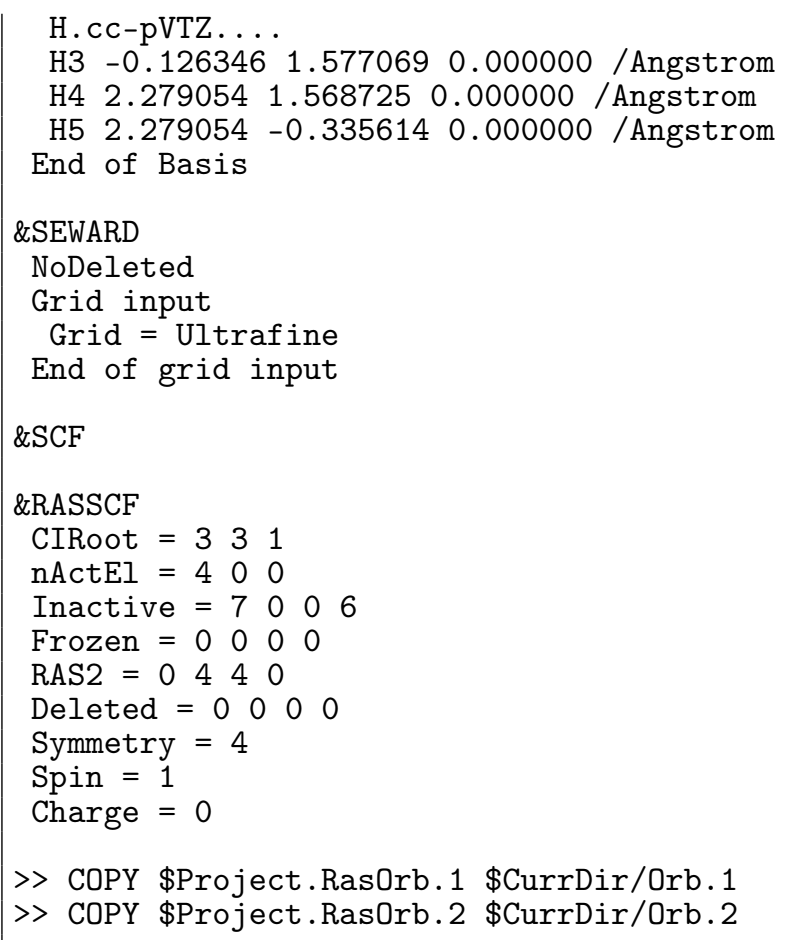

Then, to print the orbital $\left\langle r^{2}\right\rangle$ for individual natural orbitals of each of the first two states, \$Project.RasOrb.1 and \$Project.RasOrb.2, use the following input:

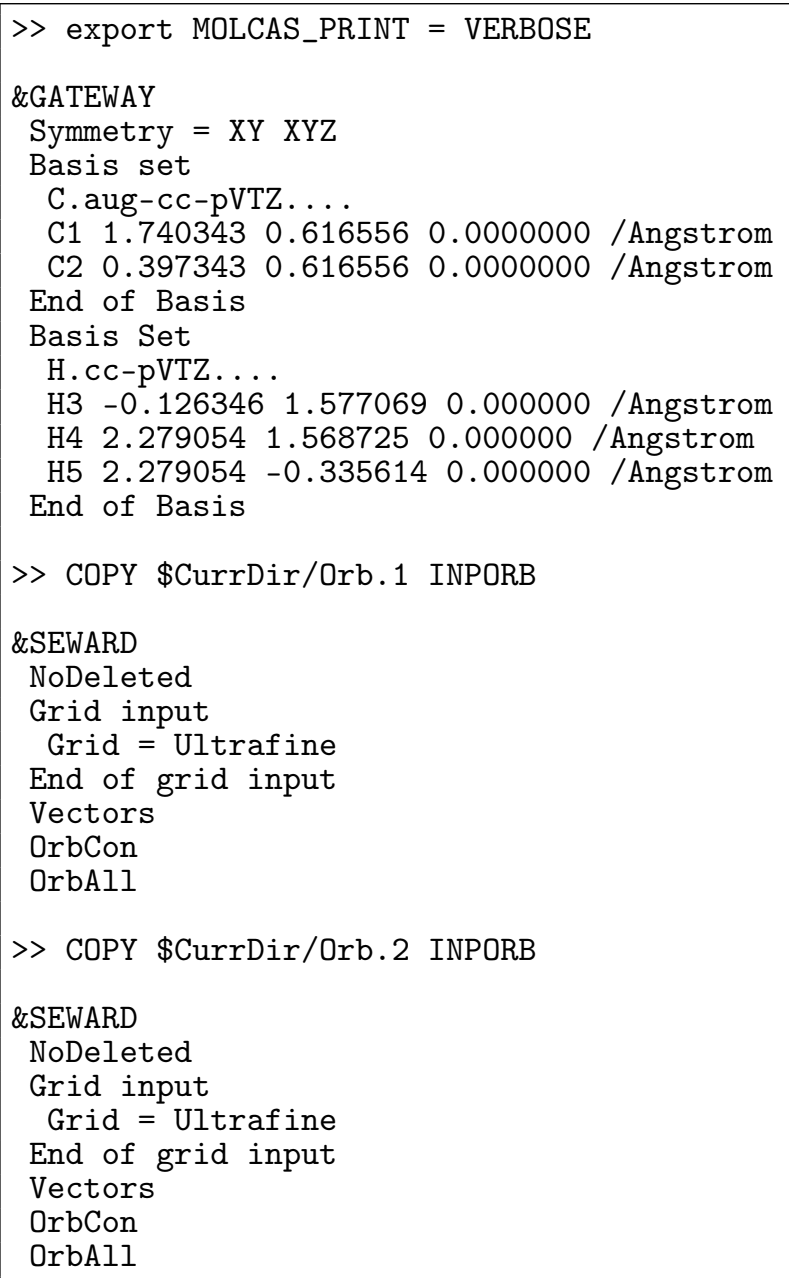

Then, the magnitude of orbital $\left\langle r^{2}\right\rangle$ and its components (especially its $\left\langle z^{2}\right\rangle$ component 
because the molecule is on the $x y$ plane) printed in the output indicates how diffuse an orbital is.

\section{Binatural orbitals}

Generation of binatural orbitals for a transition between two anion states of pentadienyldithiol (PDDT).

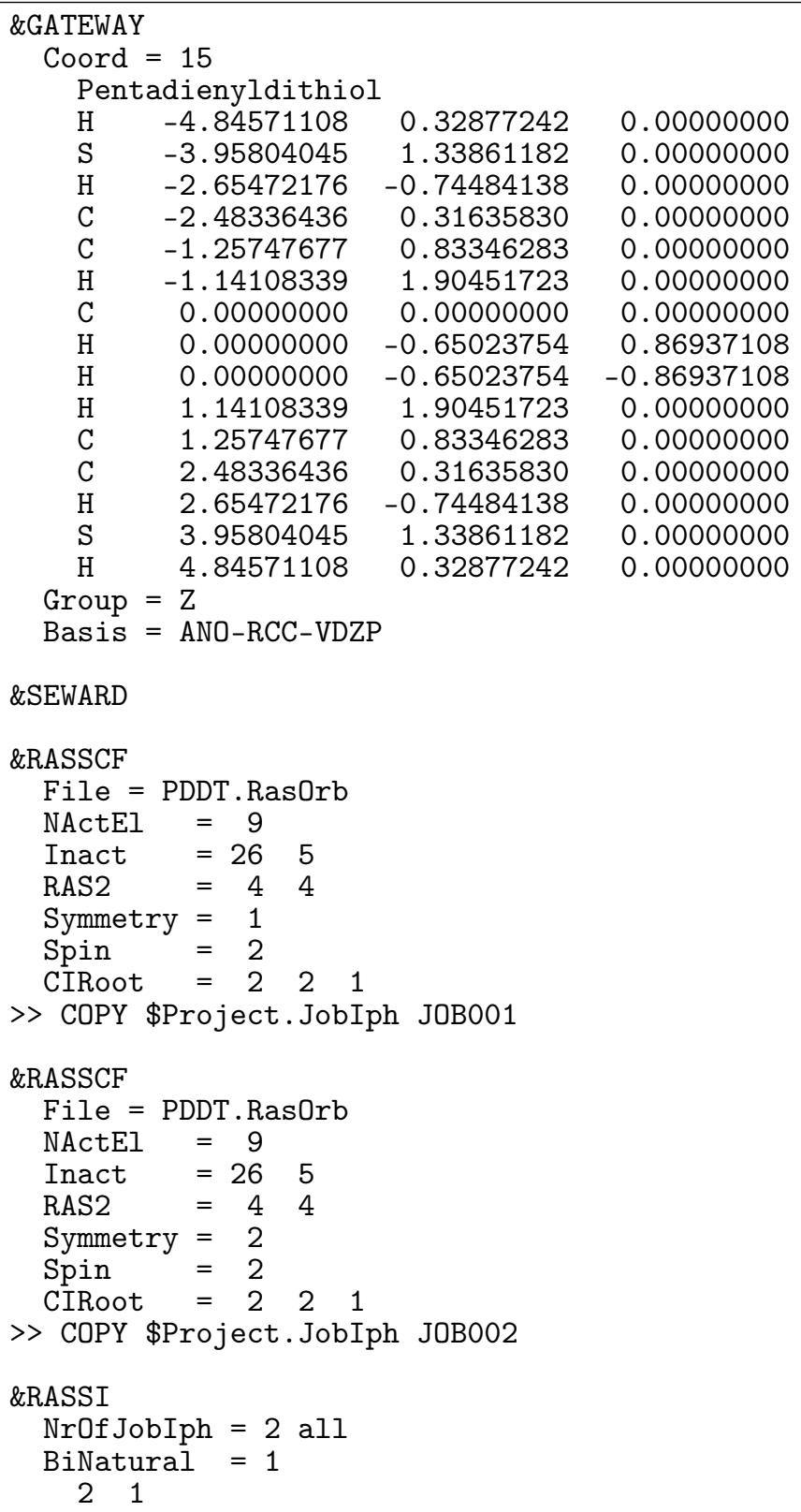

\section{The WFA module}

\begin{tabular}{|llr}
\hline \&GATEWAY \\
Coord $=4$
\end{tabular}
$\begin{array}{crrr}\text { C1 } & 0.000000000 & & \\
03 & 0.000000000 & 0.000000000 & 0.592249000 \\
& 0.000000000 & -0.595374000\end{array}$




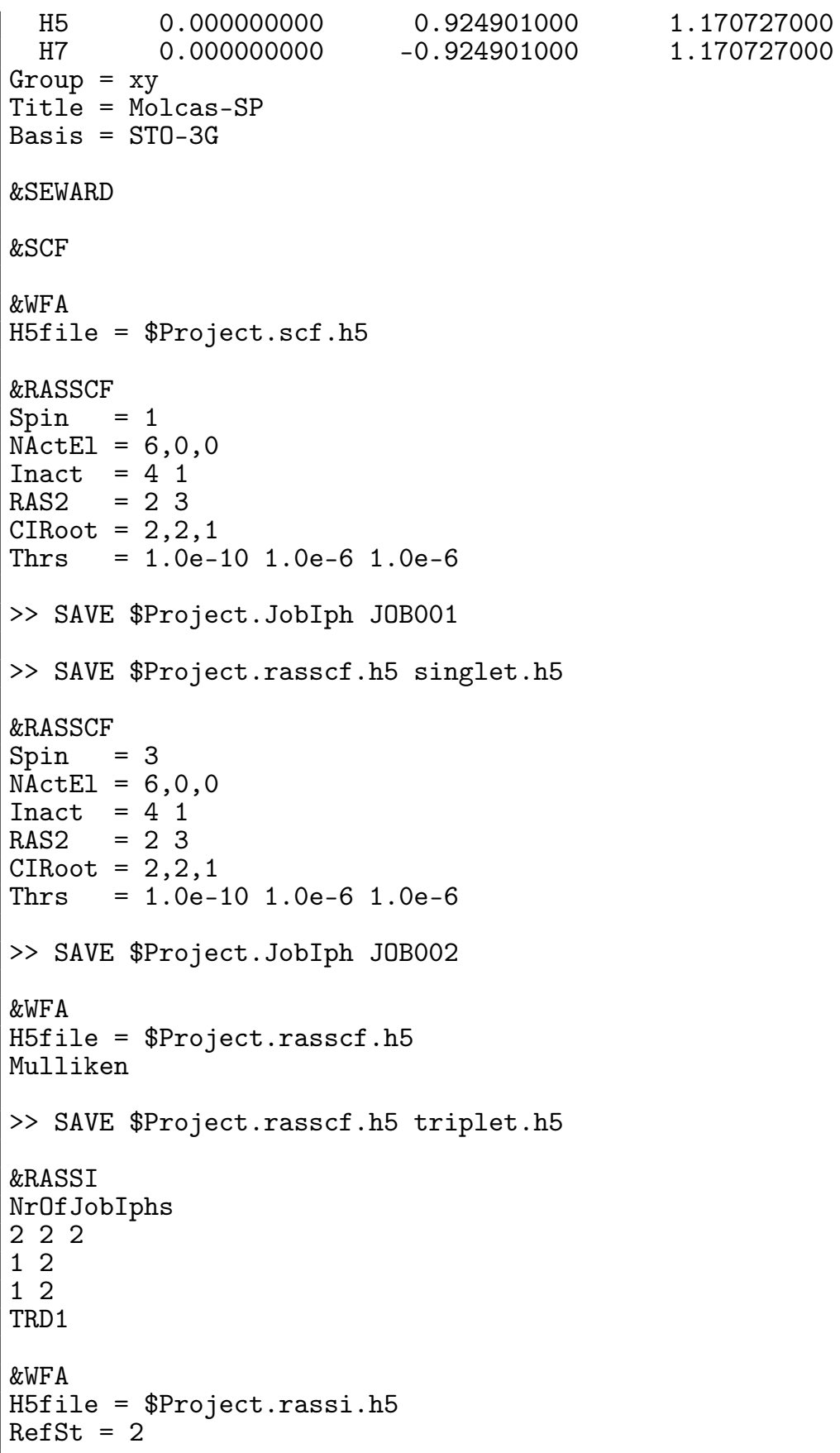

\section{Example of FDET calculation}

We will briefly describe the OpenMolcas input for a FDET calculation aiming at reproducing the effect of the presence of a nearby water molecule on the excitation energies of $\mathrm{Br}_{2}$. Following the flow-chart of fig. S8, we see that the input requires the definition of the full $A+B$ system in GATEWAY. Note the necessity of keywords such as NOMOVE and GROUP, as well as the appropriate usage of the BSSE keyword that defines the system for which the density will be generated in the subsequent SCF calculation. In this example, a PBE density is produced for the subsystem $B$ and stored with additional information on the file AUXRFIL. In the second call to GATEWAY, the role of $A$ and $B$ with respect to the keyword BSSE is exchanged, as we are now going to compute CAS-CI 
wavefunctions for $A$ embedded in $B$. Note the use of the keyword FAKE in SEWARD to avoid recomputing Cholesky vectors for the AO basis. The first RASSCF run in the input generates $\rho_{A}^{\text {ref }}(\boldsymbol{r})$ (ground-state CAS-CI) for the subsequent linearized FDET calculation. The latter will be automatically activated by the presence of the file PRERFIL that stores information about $\rho_{A}^{\mathrm{ref}}(\boldsymbol{r})$. FDET is introduced in the second RASSCF run with the keyword OFEM, that stands for orbital-free embedding potential. This keyword requires a string with two arguments, representing the functional for $T_{s}^{\text {nad }}\left[\rho_{A}, \rho_{B}\right]$ and $E_{\mathrm{xc}}^{\text {nad }}\left[\rho_{A}, \rho_{B}\right]$, respectively, separated by a slash. In the present input, LDTF/PBE indicates that the Thomas-Fermi functional is used for $T_{s}^{\text {nad }}\left[\rho_{A}, \rho_{B}\right]$, whereas PBE is chosen for $E_{\mathrm{xc}}^{\text {nad }}\left[\rho_{A}, \rho_{B}\right]$. Finally, the CASPT2 run with OFEM uses also the keyword GHOST in order to eliminate from the first-order interacting space any excitation to orbitals localized onto the subsystem B. The threshold value chosen of 0.1 includes in the correlation treatment any orbital with a Mulliken-type population higher than $10 \%$ on subsystem $A$.

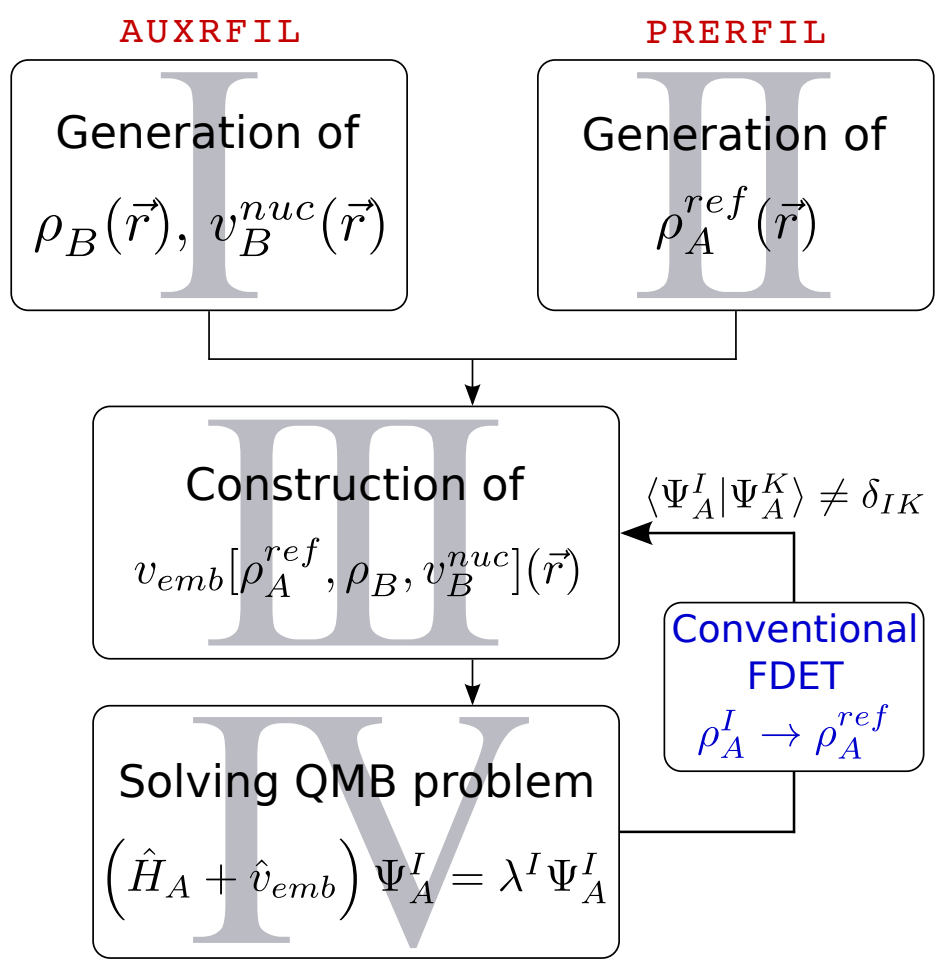

Figure S8: Flow-chart of the computational building blocks for a typical FDET calculation in OpenMolcas. The iterative update of $\rho_{A}^{\mathrm{ref}}(\boldsymbol{r})$ is not needed when using linearized FDET. $\$$ Finthermore, it should be noted that for a given electronic state $I$ of the embedded subsystem $A$, the corresponding eigenvalue $\lambda^{I}$ of the embedded Hamiltonian does not represent the value for the energy functional that is minimized in FDET, as a consequence of the inhomogeneity of the nonadditive kinetic and exchange-correlation functionals.. S13|S14

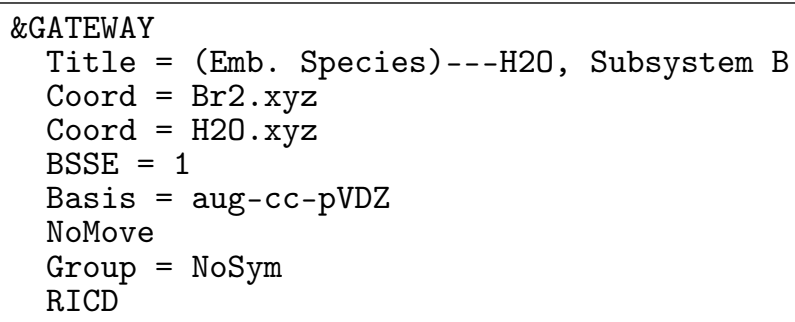




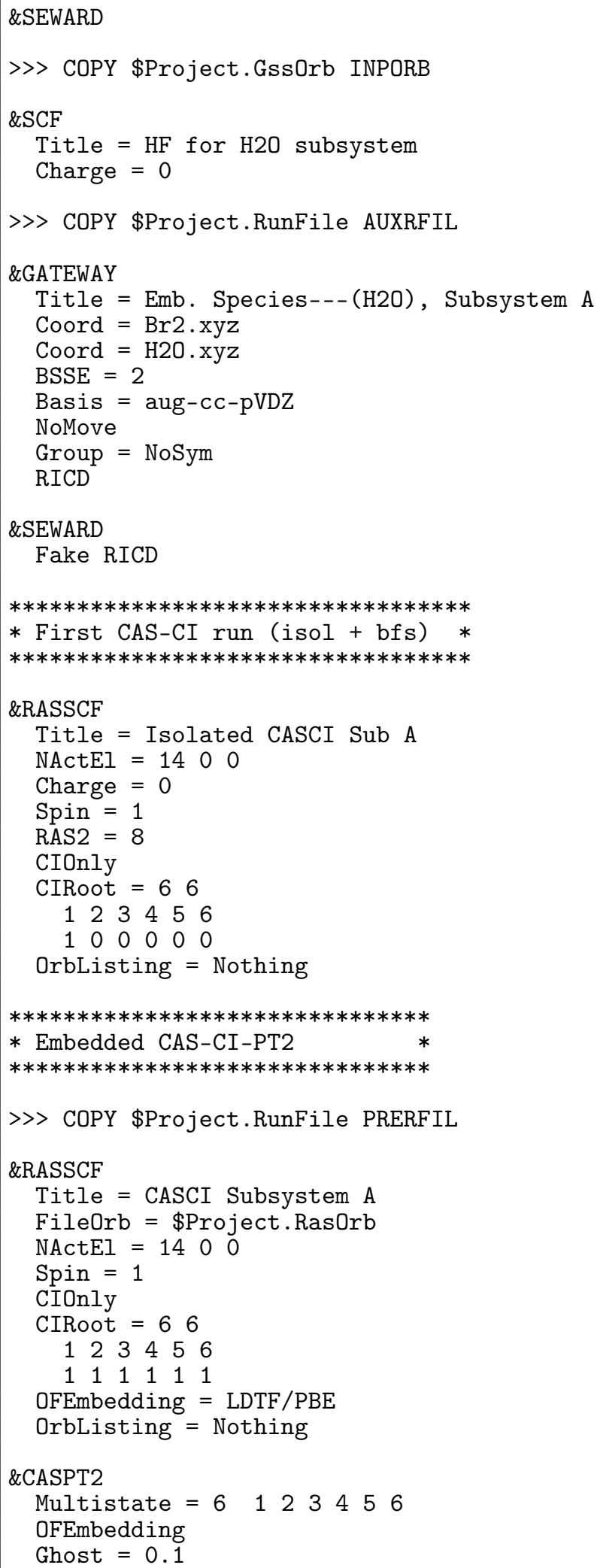




\section{References}

(S1) Wouters, S.; Van Speybroeck, V.; Van Neck, D. DMRG-CASPT2 study of the longitudinal static second hyperpolarizability of all-trans polyenes. J. Chem. Phys. 2016, 145,054120 .

(S2) Kurashige, Y.; Yanai, T. Second-order perturbation theory with a density matrix renormalization group self-consistent field reference function: Theory and application to the study of chromium dimer. J. Chem. Phys. 2011, 135, 094104.

(S3) Harding, M. E.; Metzroth, T.; Gauss, J.; Auer, A. A. Parallel Calculation of CCSD and CCSD(T) Analytic First and Second Derivatives. J. Chem. Theory Comput. 2008, 4, 64-74.

(S4) Guo, Y.; Sivalingam, K.; Valeev, E. F.; Neese, F. SparseMaps-A systematic infrastructure for reduced-scaling electronic structure methods. III. Linear-scaling multireference domain-based pair natural orbital $N$-electron valence perturbation theory. J. Chem. Phys. 2016, 144, 094111.

(S5) Roos, B. O.; Lindh, R.; Malmqvist, P.-A.; Veryazov, V.; Widmark, P.-O. New Relativistic ANO Basis Sets for Transition Metal Atoms. J. Phys. Chem. A 2005, 109, 6575-6579.

(S6) Aquilante, F.; Gagliardi, L.; Pedersen, T. B.; Lindh, R. Atomic Cholesky decompositions: A route to unbiased auxiliary basis sets for density fitting approximation with tunable accuracy and efficiency. J. Chem. Phys. 2009, 130, 154107.

(S7) Aquilante, F.; Pedersen, T. B.; Lindh, R.; Roos, B. O.; Sánchez de Merás, A.; Koch, H. Accurate ab initio density fitting for multiconfigurational self-consistent field methods. J. Chem. Phys. 2008, 129, 024113.

(S8) Aquilante, F.; Malmqvist, P.-Å.; Pedersen, T. B.; Ghosh, A.; Roos, B. O. Cholesky Decomposition-Based Multiconfiguration Second-Order Perturbation Theory (CDCASPT2): Application to the Spin-State Energetics of $\mathrm{Co}^{\mathrm{III}}$ (diiminato) $(\mathrm{NPh})$. J. Chem. Theory Comput. 2008, 4, 694-702.

(S9) Hess, B. A. Relativistic electronic-structure calculations employing a twocomponent no-pair formalism with external-field projection operators. Phys. Rev. A 1986, 33, 3742-3748.

(S10) Wolf, A.; Reiher, M.; Hess, B. A. The generalized Douglas-Kroll transformation. J. Chem. Phys. 2002, 117, 9215-9226.

(S11) Reiher, M.; Wolf, A. Exact decoupling of the Dirac Hamiltonian. II. The generalized Douglas-Kroll-Hess transformation up to arbitrary order. J. Chem. Phys. 2004, 121, 10945.

(S12) Zech, A.; Aquilante, F.; Wesolowski, T. A. Orthogonality of embedded wave functions for different states in frozen-density embedding theory. J. Chem. Phys. 2015, $143,164106$. 
(S13) Zech, A.; Aquilante, F.; Wesolowski, T. A. Homogeneity properties of the embedding potential in frozen-density embedding theory. Mol. Phys. 2015, 114, 1199 1206.

(S14) Wesołowski, T. A. Embedding a multideterminantal wave function in an orbitalfree environment. Phys. Rev. A 2008, 77, 012504. 
Other files

v2_190906_SI_input.zip (125.28 KiB)

view on ChemRxiv - download file 